\begin{tabular}{|c|c|}
\hline $\begin{array}{l}\text { 2. To: (Receiving organization) } \\
\text { WRAP } 1 \text { Engineering }\end{array}$ & $\begin{array}{ll}\text { 3. From: } & \text { criginating Organi } \\
\text { WRAP 1 Engineering }\end{array}$ \\
\hline $\begin{array}{l}\text { 5. Proj./Prog./Dept./Div.: } \\
\text { Org Code: } 32620\end{array}$ & $\begin{array}{l}\text { 6. Design Authority/ Design A } \\
\text { Engr.: } \\
\text { J. R. Weidert }\end{array}$ \\
\hline
\end{tabular}

11. Receiver Remarks: 11A. Design Baseline Document? [] Yes [X] No

zation)

on)

(10.

\begin{tabular}{|} 
4. Related EDT No.: \\
N/A
\end{tabular}

9. Equip./Component No.: WRAP 1 DMS

10. System/Bldg-/Facility: N/A

12. Major Assmi. Dwg. No.: $\mathrm{N} / \mathrm{A}$

13. Permit/Permit Application No.: $N / A$

14. Required Response Date:

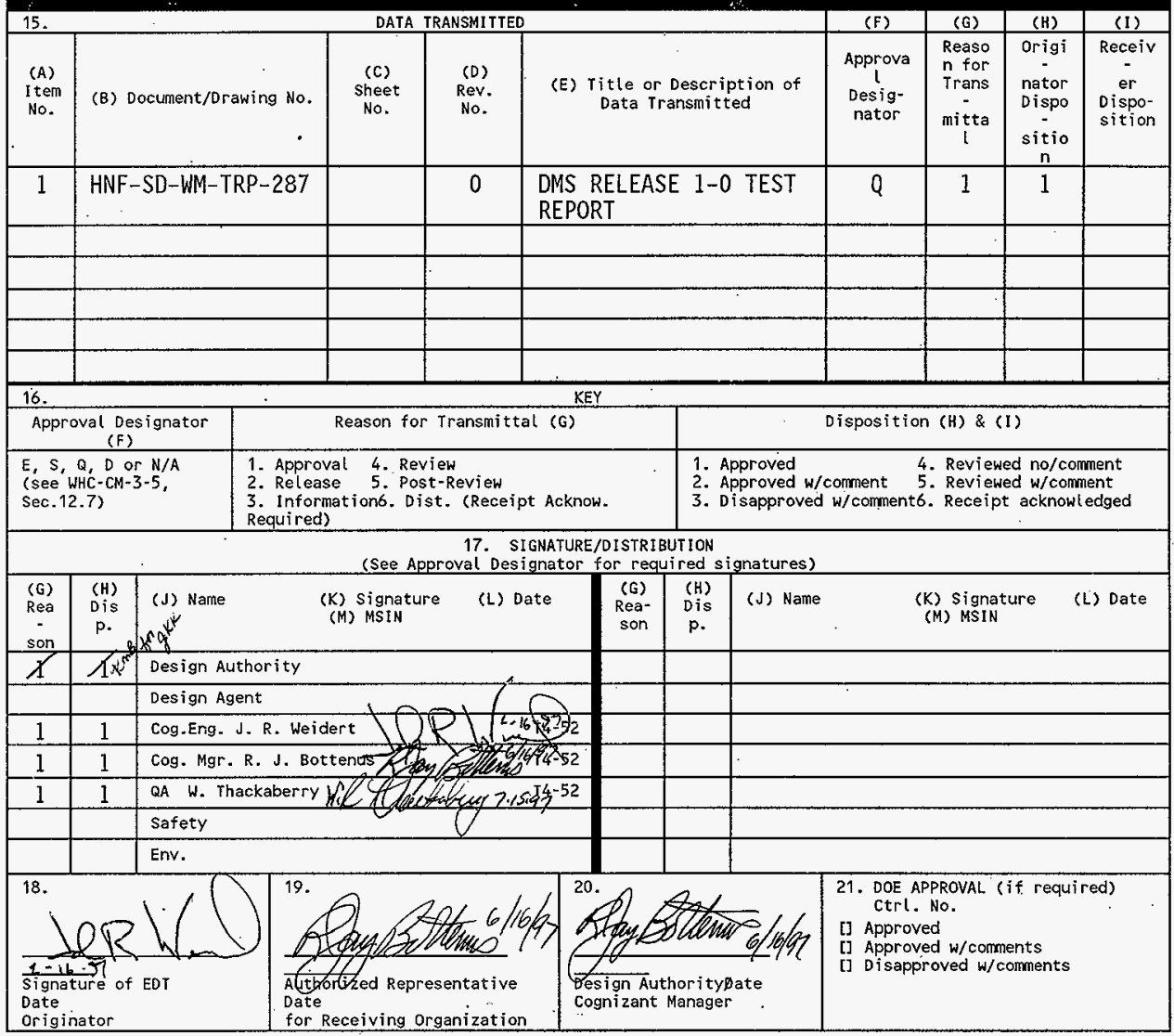




\title{
WRAP Module 1 Data Management System SOFTWARE TEST REPORT
}

\author{
JR Weidert \\ U.S. Department of Energy Contract DE-AC06-96RL13200

$\begin{array}{llll}\text { EDT/ECN: } & \text { EDT } 620586 & \text { UC: } 506 \\ \text { Org Code: } & 32620 & \text { Charge Code: } & \text { A41B3 } \\ \text { B\&R Code: } & \text { EW3130020 } & \text { Tota1 Pages: } & 185\end{array}$

Rust Federal Services of Hanford, Inc., Richland, WA 99352

Key Words: W-026, DMS, SWITS, SIE, PCS, Computer

Abstract: This document summarizes the test result information for the Data Management System (DMS). Appendix A contains test result information for all Functional Test cases and Appendix $B$ contains the results for all the Performance Test cases.

TRADEMARK DISCLAIMER. Reference herein to any specific comercial product, process, or service by trade name, trademark, manufacturer, or otherwise, does not necessarily constitute or imply its endorsement, recommendation, or favoring by the United States Government or any agency thereof or its contractors or subcontractors.

Printed in the United States of America. To obtain copies of this document, contact: Document Control Services, P.O. Box 950, Mailstop H6-08, Richland WA 99352, Phone (509) 372-2420; Fax (509) 376-4989.
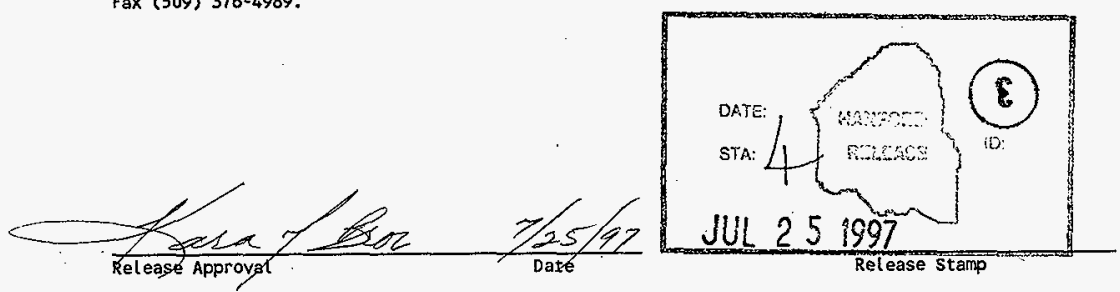
Supporting Document Coversheet HNF-SD-WM-TRP-287, Rev. 0

P.age 2 of 2

*Paradox is a registered trademark of Borland, International, Scotts Valley, CA.

*WordPerfect is a registered trademark of Wordperfect Corp., Orem, UT. *0racle is a registered trademark of Oracle Corp., Redwood Shores, CA. *HP 9000 is a registered trademark of Hewlett-Packard, Inc., San Jose, CA. *HP-UX is a registered trademark of Hewlett-Packard, Inc, San Jose, CA.

*IBM PC is a registered trademark of International Business Machine, Corporation, Buffalo, NY.

*MS-Dos \& MS-Windows are registered trademarks of Microsoft Corporation, Seattle, WA.

*TeamTest is a registered trademark of Software Quality Automation, Inc., Woburn, MA. 
WRAP Module 1 Data Management System

\section{SOFTWARE TEST REPORT}

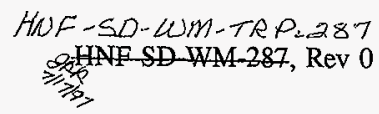

June 13, 1997

Issued By:

Rust Federal Services, Hanford

for the

United States Department of Energy, Richland Operations Office Richland, Washington

Prepared by:

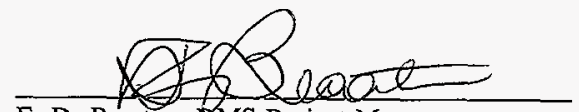

F. D. Braaten, DMS Project Manager

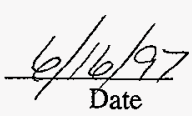

Contractor Approval:
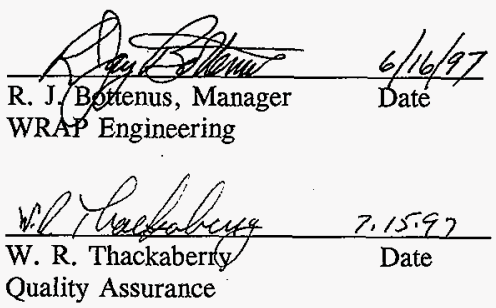

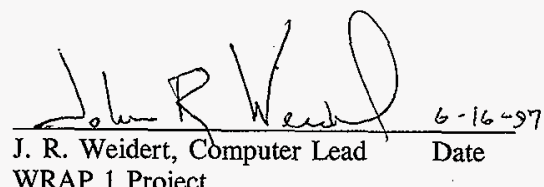

WRAP 1 Project 


\section{TABLE OF CONTENTS}

\section{Page}

1.0 SUMMARY

1.1 Testing Conducted

1.2 Testing Environment

1.3 Phase 1 Readiness Assessment

1.4 References

2.0 VARIANCES 2

3.0 COMPREHENSIVE ASSESSMENT

4.0 SUMMARY OF RESULTS/EVALUATION 


\subsection{SUMMARY}

\subsection{Testing Conducted}

The development team programmers assumed responsibility for the conduct and recording of unit testing. Once the unit tests were completed, WHC personnel conducted functional and performance testing:

Functional Tests: Functional tests each tested a specific software module or function, such as individual screens or a communication interfaces. The software was tested against the functional requirements, including error detection and recovery. There were a total of 33 functional test procedures.

Performance Tests: Performance tests provided an integrated test of the DMS modules or functions which support specific plant activities (for example, a performance test might test all aspects of DMS support to receipt of low level waste (LLW) drums for processing). Performance tests focused on a larger system operation view and were not intended to repeat the extensive testing of the individual functions as tested by the functional tests. There were a total of 11 performance test procedures:

For all tests, exceptions/discrepancies were recorded in a central discrepancy data base. Resolution actions needed were noted. When the resolution actions were completed, the test step/case was conducted again. This was repeated as often as was necessary until the discrepancy was corrected and the test step/case passed. The data base was then updated to indicate the discrepancy was closed.

Testers noted on their test procedures that a test step was passed. Once all steps in the test procedure passed, the procedure was signed off and filed in the test file.

\subsection{Testing Environment}

Testing was conducted on the central DMS file server, accessed from remote terminals. A separate test environment was created, with a data base structure copied from the development environment. Test data was created to replicate typical test data expected during WRAP facility operations. Data was also loaded directly from the SWITS data base as will occur during actual DMS operations. Message communication with the Plant Control System (PCS), and the System Integration Equipment (SIE) was exercised using PCS and SIE simulators which could receive messages, or generate messages, as appropriate to the DMS system needs at a particular point of processing. 


\subsection{Phase 1 Readiness Assessment}

Based on the test results, Release 1 of DMS is considered ready for plant implementation. The entire DMS system is still in a state of development, and Release 2 of DMS will be implemented at a later date. In the interim, the DMS Release 1 software will be exercised in the WRAP facility in an integrated environment with actual PCS and SIE software. This activity may lead to new discovery causing later changes to Release 1 . The intent of testing to date was to gain assurance that the DMS software was ready to support this stage of development. The functional and performance test results indicate that this has been achieved.

\subsection{References}

a. WHC-CM-W026-SRS-001, DMS System Requirements Specifications

b. WHC-CM-W026-SDD-001, DMS System Design Document

c. DMS Phase 1 Test Plan (Attached as Appendix. A).

d. Test Procedures/Test Cases

The original signed test cases are filed in the DMS Project Files and are available for review. It is not practical to include all of them in this report. They are summarized in later paragraphs of this report. Appendix D contains a typical sample of a functional and performance test procedure. The originals are available for review upon request.

e. Test Logs/Test. Incident Report

Discrepancies are noted in a PARADOX data base accessible on a central file server. Discrepancy reports in Word Perfect format are also on the central file server and are available for review. Appendix E contains a typical test discrepancy report. The originals are available for review upon request.

\subsection{VARIANCES}

The actual sequence and schedule of the testing varied significantly from the original test plan. This was a reaction to the demands of discrepancy rework and actual development activity scheduling which impacted developer resources. However, all tests were conducted and signed off for approval. 
activity scheduling which impacted developer resources. However, all tests were conducted and signed off for approval.

The following test steps were approved with exceptions. The disposition of each exception is noted:

\begin{tabular}{|c|c|c|c|c|}
\hline CASE & STEP & STEP TITLE & EXCEPTION & DISPOSITION \\
\hline $\begin{array}{l}\text { DMSF- } \\
14.01 \\
\text { and } \\
\text { DMSF- } \\
14.02\end{array}$ & 92 & $\begin{array}{l}\text { Edit PIN number displayed and } \\
\text { confirm validity (waste drum } \\
\text { PIN with no previous NDE } \\
\text { records, not at NDE vault } \\
\text { location): }\end{array}$ & $\begin{array}{l}\text { User can modify the PIN number } \\
\text { provided by DMSCOM for } \\
\text { container present in the vault }\end{array}$ & $\begin{array}{l}\text { Appears to be an } \\
\text { ORACLE } \\
\text { idiosyncrasy. Fix } \\
\text { would be costly. } \\
\text { Accept as-is }\end{array}$ \\
\hline $\begin{array}{l}\text { DMSF- } \\
14.01 \\
\text { and } \\
\text { DMSF- } \\
14.02\end{array}$ & 99 & Test [Value A] button: & $\begin{array}{l}\text { Screen would not commit a } \\
\text { record for an invalid PIN number } \\
\text { (valid PIN in system but for an } \\
\text { empty container, not for a waste } \\
\text { container) but would not allow } \\
\text { record to be deleted }\end{array}$ & $\begin{array}{l}\text { Use the Menu Bar } \\
\text { function, 'Record } \\
\text { Clear' as a } \\
\text { workaround and accept } \\
\text { as-is }\end{array}$ \\
\hline $\begin{array}{l}\text { DMSF- } \\
15.01\end{array}$ & 27 & $\begin{array}{l}\text { Test Isotopic Confidence Check } \\
\text { field for Autohint: }\end{array}$ & $\begin{array}{l}\text { Unable to click on data field and } \\
\text { cannot determine Autohint unless } \\
\text { displayed PIN has } \\
\text { CONNECT_USE_CD of 'WV' }\end{array}$ & $\begin{array}{l}\text { Fields are not } \\
\text { clickable because of } \\
\text { requirement not to } \\
\text { display the data except } \\
\text { the 'WV' drums. No } \\
\text { change possible with } \\
\text { Forms } 4.0 \text { version of } \\
\text { ORACLE. Accept as- } \\
\text { is }\end{array}$ \\
\hline $\begin{array}{l}\text { DMSF- } \\
15.01\end{array}$ & 28 & $\begin{array}{l}\text { Test Total PE Ci field for } \\
\text { Autohint: }\end{array}$ & $\begin{array}{l}\text { Unable to click on data field and } \\
\text { cannot determine Autohint unless } \\
\text { displayed PIN has } \\
\text { CONNECT_USE_CD of 'WV' }\end{array}$ & $\begin{array}{l}\text { Fields are not } \\
\text { clickable because of } \\
\text { requirement not to } \\
\text { display the data except } \\
\text { the 'WV' drums. No } \\
\text { change possible with } \\
\text { Forms } 4.0 \text { version of } \\
\text { ORACLE. Accept as- } \\
\text { is }\end{array}$ \\
\hline
\end{tabular}




\begin{tabular}{|c|c|c|c|c|}
\hline CASE & STEP & STEP TTTLE & EXCEPTION & DISPOSITION \\
\hline $\begin{array}{l}\text { DMSF- } \\
15.01\end{array}$ & 29 & $\begin{array}{l}\text { Test Total FGE field for } \\
\text { Autohint: }\end{array}$ & $\begin{array}{l}\text { Unable to click on data field and } \\
\text { cannot determine Autohint unless } \\
\text { displayed PIN has } \\
\text { CONNECT_USE_CD of 'WV' }\end{array}$ & $\begin{array}{l}\text { Fields are not } \\
\text { clickable because of } \\
\text { requirement not to } \\
\text { display the data except } \\
\text { the 'WV' drums. No } \\
\text { change possible with } \\
\text { Forms } 4.0 \text { version of } \\
\text { ORACLE. Accept as- } \\
\text { is }\end{array}$ \\
\hline $\begin{array}{l}\text { DMSF- } \\
15.01\end{array}$ & 30 & $\begin{array}{l}\text { Test Thermal Power field for } \\
\text { Autobint: }\end{array}$ & $\begin{array}{l}\text { Unable to click on data field and } \\
\text { cannot determine Autohint unless } \\
\text { displayed PIN has } \\
\text { CONNECT_USE_CD of 'WV' }\end{array}$ & $\begin{array}{l}\text { Fields are not } \\
\text { clickable because of } \\
\text { requirement not to } \\
\text { display the data except } \\
\text { the 'WV' drums. No } \\
\text { change possible with } \\
\text { Forms } 4.0 \text { version of } \\
\text { ORACLE. Accept as- } \\
\text { is }\end{array}$ \\
\hline $\begin{array}{l}\text { DMSF- } \\
15.01\end{array}$ & 31 & Test [NDS Results] button: & $\begin{array}{l}\text { On the NDA Results pop-up, the } \\
\text { assay date field is highlighted } \\
\text { (PIN number should be } \\
\text { highlighted). Inconsistent with } \\
\text { other screens }\end{array}$ & $\begin{array}{l}\text { Accept as-is for Phase } \\
\text { To be Resolved after } \\
\text { Startup if identified as } \\
\text { a problem by OPS }\end{array}$ \\
\hline $\begin{array}{l}\text { DMSF- } \\
15.01\end{array}$ & 34 & $\begin{array}{l}\text { Test [Isotopic Data] button for } \\
\text { selected PIN: }\end{array}$ & $\begin{array}{l}\text { On the NDA ISO screen, the } \\
\text { assay date field is highlighted } \\
\text { (PIN number should be } \\
\text { highlighted). Inconsistent with } \\
\text { other screens }\end{array}$ & $\begin{array}{l}\text { Accept as-is be To be } \\
\text { Resolved after Startup } \\
\text { if identified as a } \\
\text { problem by OPS }\end{array}$ \\
\hline $\begin{array}{l}\text { DMSF- } \\
15.01\end{array}$ & 43 & Test [Request Revisit] button: & $\begin{array}{l}\text { The 'IS11 Send to SIE Complete' } \\
\text { message is displayed on the } \\
\text { status bar and not as a message } \\
\text { box. Inconsistent with other } \\
\text { messages }\end{array}$ & $\begin{array}{l}\text { Accept as-is for Phase } \\
\text { To be Resolved after } \\
\text { Startup if identified as } \\
\text { a problem by OPS }\end{array}$ \\
\hline $\begin{array}{l}\text { DMSF- } \\
17.03\end{array}$ & 15 & $\begin{array}{l}\text { Test Group field for editability, } \\
\text { length, case restriction, and valid } \\
\text { data values: }\end{array}$ & $\begin{array}{l}\text { When an invalid entry is made } \\
\text { and the LOV is canceled, } \\
\text { message FRM- } 40212 \text { is displayed } \\
\text { in message box. Other screens } \\
\text { display message on the status bar }\end{array}$ & $\begin{array}{l}\text { Accept as-is for } \mathrm{Phase} \\
\text { To be Resolved after } \\
\text { Startup if identified as } \\
\text { a problem by OPS }\end{array}$ \\
\hline
\end{tabular}




\begin{tabular}{|l|l|l|l|l|}
\hline CASE & $\begin{array}{c}\text { STE } \\
\mathbf{P}\end{array}$ & \multicolumn{1}{|c|}{ STEP TITLE } & EXCEPTION & DISPOSITION \\
\hline $\begin{array}{l}\text { DMSP- } \\
21.01\end{array}$ & 7 & $\begin{array}{l}\text { Radiologic Inventory Summary } \\
\text { Screen (DMSS1101): }\end{array}$ & $\begin{array}{l}\text { The 202 screen 'Compliant' field } \\
\text { does not display the data for the } \\
\text { inner drum }\end{array}$ & $\begin{array}{l}\text { Accept as-is. To be } \\
\text { worked on SCR } 84\end{array}$ \\
\hline
\end{tabular}

\subsection{COMPREHENSIVE ASSESSMENT}

The DMS Release 1 testing was very thorough. No approved Release 1 requirements were omitted, including final "as-built" approved design changes. All tests were considered to be comprehensive for the test step involved.

\subsection{SUMMARY OF RESULTS/EVALUATION}

Appendix A contains test result information for all Functional Test cases. Appendix B contains test result information for all Performance Test cases. These appendices show the test case, all of the discreet test steps which were conducted, the version number of the test case, whether the test step passed the first test or a follow-on retest, and the date the test step was signed off as being successfully passed.

Given the state of DMS development, Release 1 is considered ready for implementation in the WRAP-1 facility. It has been shown to be functional enough to support the continued planned development activities for DMS.

Risk of failure is not being assessed at this point of the DMS testing. The intent for the next phases of development is to try and cause the Release 1 software fail in order to further resolve any redesign and development requirements for Release 2. This approach permits such failures to occur in a non-critical operation environment while addressing new discovery of "real world" impacts. 
HNF-SD=WM-TRP-287, Rev. 0

\section{APPENDIX A \\ Software Test Plan}




$$
\text { WHC-SD-W026-TP-010, Rev. } 0
$$

\author{
WRAP ModuTe 1 Data Management System \\ SOFTWARE TEST PLAN \\ WHC-SD-W026-TP-010, Rev 0 \\ September 1, 1995
}

Issued By:

Westinghouse Hanford Company

for the

United States Department of Energy, Richland Operations Office Richland, Washington

Prepared by:

$$
\text { C. K. Rosnick, Software Engineer }
$$

Contractor Approval:

J. E. Filip, Manager Date WRAP I Project

R. J. Bottenus, Manager New Facilities Startup

R. E. Clark, Project Manager Date ISS Environmental Systems
D. A. Guettler, Manager Date ISS EnvironmentaT Systems

J.R. Weidert, Project Engineer Date WRAP 1 Project

J. R. McGee

QuaTity Assurance
Date 
WHC-SD-W026-TP-010, Rev. 0

DMS SYSTEM TEST PLAN CONTENTS

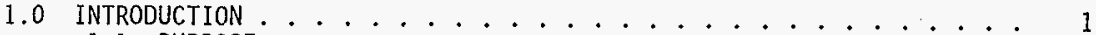

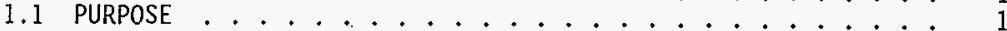

1.2 SCOPE .................... . . . . . 1

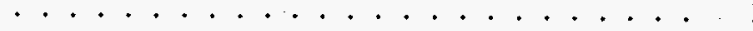

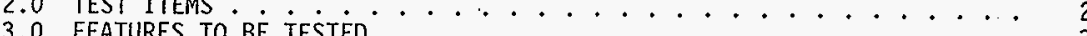

3.0 FEATURES TO BE TESTED . . . . . . . . . . . . . . . . 3

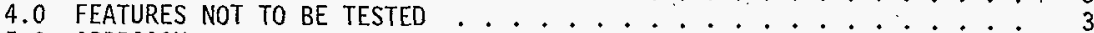

5.0 APPROACH . . . . . . . . . . . . . . . . . . . . . . 3

6.0 ITEM PASS/FAIL CRITERIA ..................... . . . . . 4

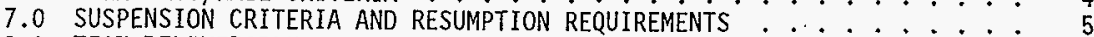

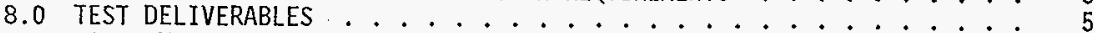

8.1 TEST LOG......................... . . . . . . 5

8.2 TEST PROCEDURES $: . . . . . . . . . . . . . . . . . . .55$

8.3 DISCREPANCY REPORTS . . . . . . . . . . . . 5

8.4 TEST REPORTS . . . . . . . . . . . . . . . 5

9.0 TESTING TASKS ........................... . . . . . . 5

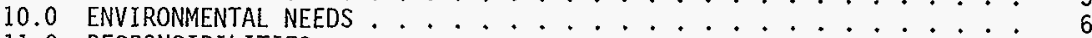

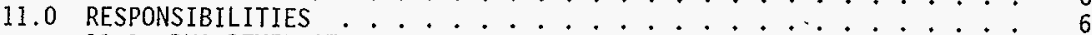

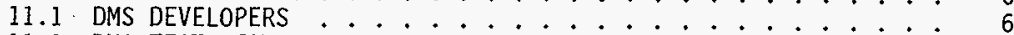

11.2 DMS TEST TEAMS ..................... . . . . . . 7

11.3 QUALITY ASSURANCE ...................... . . . . . 7

12.0 STAFFING AND TRAINING . . . . . . . . . . . . . . . . . . . . . . 7

12.1 TEST CASE PREPARATION ................ . . . 7

12.2 SYSTEM OPERATION AND MAINTENANCE .............. . . . . 7

12.3 TEST EXECUTION . . . . . . . . . . . . . . . . 7

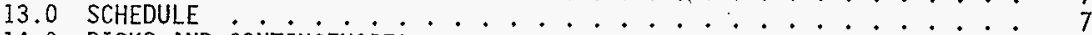

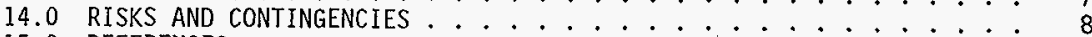

15.0 REFERENCES ......................... . . . . . . . . . 8

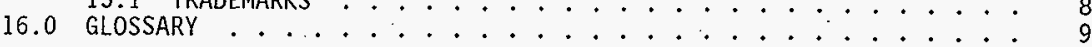

FIGURE 1: DMS TEST LOG . . . . . . . . . . . . . . . . . . 11

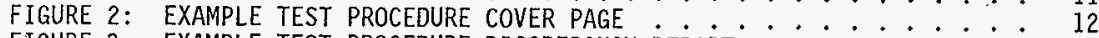

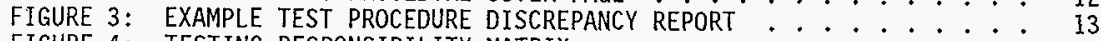

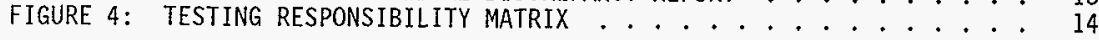


WHC-SD-W026-TP-010, Rev, 0

\subsection{INTRODUCTION}

\subsection{PURPOSE}

This test plan describes the scope, approach, activjties, resources, and schedule for testing of the WRAP DMS software deveToped by BCSR for the WRAP 1 Project, $W-026$.

\subsection{SCOPE}

The scope of the testing covered under this plan is the testing to be performed prior to acceptance of the software by the WRAP 1 Project. This includes software module unit-testing, interface testing, functional testing, integration testing, and formal shop and site acceptance testing. The plan does not cover operational testing.

The software to be tested is being developed by BCSR under the WRAP. Facility Module 1 DMS Software Project Management P7an (SPMP), WHC-SD-W026SDP-001, Rev. 0, November 16, 1994. The requirements are documented in WRAP Modu7e 1 DMS Software Requirements Specification, Rev. 0, September 19, 1994 and the software design is documented in WRAP Module 1 DMS Software Design Description, Rev, 0, March 1995.

This plan covers the testing of the set of software modules identified for DMS Phase 1 implementation in the June 1995 addendum to the SPMP. The test plan will be revised as additional modules are authorized and scheduled for implementation.

\subsection{OVERVIEW}

The DMS is one of the plant computer systems for the WRAP 1 facility, which is being constructed under Westinghouse Hanford Company Project W-026. It is a client server system with an HP 9000 E-35 server and IBM PC and Xterminal clients. The DMS will collect, store, and report data related to WRAP 1 receipt, processing, analysis, packaging, certification, and shipment of Hanford contact-handled retrieved and newly-generated low-level and transuranic (TRU) waste.

Waste characterization and certification data required for WRAP 1 processing will be downloaded from the Solid Waste Information and Tracking System (SWITS) when waste is received. The data will be updated on the DMS to reflect WRAP 1 processing and repacking. Data will be received from the PCS, non-destructive assay (NDA) systems, and terminals located in the process and office areas. The DMS will generate waste certification and shipping reports for WRAP 1. Waste certification and shipping data will be uploaded to SWITS when the waste is shipped. The data associated with shipped items will be stored on the DMS for several months and then archived to magnetic tape. 
WHC-SD-W026-TP-010, Rev. 0

\subsection{TEST ITEMS}

The following software items are to be tested:

- DMS Communication module (DMSCOM)

- User logon and menu screens

- Receiving Screens

- DMSS0101 Container Receiving

- DMSS0102 Bar Code

- NDE/NDA Operations screens

- DMSS0201 NDE

- DMSS0202 NDA

- LLW Process GTovebox screens

- DMSS0311 LLW Drum Status at Entry

- DMSS0312 LLW Sorting Table

- DMSS0315 LLW Exit GTovebox

- Data Review screens (Part)

- DMSS1221 Processed Waste Data Review

- Shipping screens (Part)

- DMSS0602 Loading Dock Container Shipping

- Picklist screens (except TRUPACT)

- DMSS0901 Download Processing List

- DMSS0902 Process Pick List

- DMSS0903 AS/RS Storage Pick List

- DMSS0904 AS/RS Shipping Pick List

- Facility Inventory Screens

- DMSS1101 Radiological Inventory Summary

- Table Maintenance screens

- DMSS0452 Location

- DMSS0454 Profile Isotopic

- DMSS0455 Route Description

- DMSS0456 Shipment Picklist Description

- DMSS0457 SIE Isotope Name

- DMSS0458 TRU Container Code

- DMSS0459 Field Analysis Type

- DMSS0460 Material

- DMSS0461 WRAP Miscel1 aneous

- DMSS0462 Laboratory Analysis

- DMSS0463 Sample Container

- DMSS0464 Sample Matrix

- DMSS0465 Sampling Method

- DMSS0466 Treatment Procedure 
WHC-SD-W026-TP-010, Rev. 0

- System Administration screens

- DMSS0701 Error Messages

- DMSS0702 User

- DMSS0703 Role

- DMSS0704 Screen

- DMSS0706 User RoTe

- DMSS0707 Role Screen

- DMSS0711 Form Help

- DMS ORACLE database

\subsection{FEATURES TO BE TESTED}

The following features will be tested:

- Security functions (logon, database access)

- Menu functions

- Screen display of selected database information

- Database activities (add/change/deTete)

- Signature password

- Pop-up screens

- Refresh button

- Logon and screen displays on PCS X-terminals

- Bar code 1 abel printing

- SWITS data download and upload

- Processing of messages to and from the PCS

- Processing of messages to and from the SIE

- Error detection and recovery

- Performance and load capabilities

\subsection{FEATURES NOT TO BE TESTED}

The items and features of the DMS not specifically addressed as part of this test plan are the system software (HP-UX, ORACLE, MS-DOS, MS-Windows), HLAN, WLAN, and computer-related hardware. These components are not part of the DMS software design and will not be tested separately.

\subsection{APPROACH}

Unit testing will be performed by the programmers as part of the software development. This testing will be performed using appropriate software stubs and test data to verify that the software will run without error, will process prescribed input data, will perform prescribed 7 ogic, and will provide prescribed output, including display screens. Prior to completion of unit testing, screens will be reviewed by the WRAP project and concurrence received on the screen layout and format. When the programmer has completed unit testing of the software item, the software will be placed under configuration control by the BCSR DMS Project Manager and available for functional testing with other software units. 
WHC-SD-W026-TP-010, Rev. 0

Test procedures will be prepared to test communication interfaces, man/machine interfaces, security features, and system performance. The test procedures will be prepared by the WRAP 1 Project and reviewed by the DMS development team. Test databases will be established for use with the test procedures. The WRAP 1 Project will define the data required for the test databases. The BCSR DMS development team will then set up the test databases on the DMS development computer and maintain the databases under configuration control. Simulation software will be used to test communication with the PCS and the SIE. The SWITS development computer will be used to test DMS interaction with the SWITS database.

The test procedures will first be run by BCSR (dry run) on the development computer to verify that the system is ready for formal testing. The test procedures may be modified at this time to correct errors. Following the shop test dry run, a formal shop test will be performed by the WRAP Project to demonstrate that the software is ready to be installed on the plant production computer. A test report will be issued by the WRAP Project. summarizing the results of the formal shop test.

Following successful execution of the shop test on the development system and acceptance of the test report by the WRAP Project, the software will be installed on the plant production computer by BCSR for formal site acceptance testing. The same test procedures will be used, except where modifications may be required to reflect differences between the development system and the plant hardware. A dry run may be run by BCSR prior to the acceptance testing by the WRAP Project. An acceptance test report will be issued by the WRAP Project summarizing the test results for the acceptance test.

\subsection{ITEM PASS/FAIL CRITERIA}

The test procedures will specify the "Expected Result" for each test step. Each failure to obtain an expected result will be documented on a "Test Procedure Discrepancy" report. There are two levels of discrepancy severity:

(1) A Level 1 discrepancy. is a major discrepancy and system testing must be terminated until the error is corrected. This level of error will prevent further testing of the system.

(2) A Level 2 discrepancy is less severe, and system testing may continue. Level 2 procedural errors may be corrected and the test proceed.

The determination of the severity level will be made by the test director with the concurrence of the test witness. 
WHC-SD-W026-TP-010, Rev. O

\subsection{SUSPENSION CRITERIA AND RESUMPTION REQUIREMENTS}

Testing will be suspended if it is deemed necessary to replace either a hardware or software item integral to the specific test. In addition, the testing will be suspended if the software fails to provide the expected result (see Section 6.0). Testing will not be resumed until the reason for the failure has been identified and corrected. The test director, with the concurrence of the test witness, will determine whether regression testing will be performed in the event software changes are made which may impact other tests.

\subsection{TEST DELIVERABLES}

\subsection{TEST LOG}

A test log will be maintained to document the results of dry run and formal shop and site testing. (see Figure 1). The test director for each test will be responsible for updating the log.

\subsection{TEST PROCEDURES}

Test cases will be defined to test all of the software items and software features during system testing. Test procedures will then be prepared for each of the test cases. Definition of the test cases and preparation of the test procedures is the responsibility of the WRAP Project. An example test procedure cover sheet is shown in Figure 2.

\subsection{DISCREPANCY REPORTS}

A discrepancy report will be completed whenever an anomaly or problem in the system is detected during shop or acceptance testing (see Figure 3 ).

\subsection{TEST REPORTS}

The shop and site system test reports will contain all the test results and discrepancy reports. The test reports will be prepared by the WRAP Project.

\subsection{TESTING TASKS}

The following tasks are necessary to prepare for and perform the system and acceptance testing.

- Identify test cases

- Evaluate the SQA TeamTest automated testing tool

- Prepare test procedures

- Identify testing responsibilities

- Schedule the testing activities

- Establish the testing environment

- Perform testing 
WHC-SD-W026-TP-010, Rev. 0

\subsection{ENVIRONMENTAL NEEDS}

The following hardware, software, and documentation are required to perform the DMS system acceptance testing. The test director is responsible for ensuring the required resources are available and in-place prior to the start of each test.

- HP-9000 Model 800/E35 computer

- HP-UX operating system

- ORACLE Data Base Management System software

- Five 486/66 PC computers, or equivatent (Minimum of three for system testing on development system)

- MS-DOS and MS Windows for Workgroups for the PC computers

- Two HP Laserjet printers

- One bar code printer

- Five X-terminals (Minimum of one for system testing on development system)

- LAN software and LAN access (TCP/IP)

- DMS appitication software

- DMS test database

- SQA TeamTest software and automated test scripts (if used)

- LAN access to SWITS development system and SWITS test database

- DMS Software Requirements Specification

- DMS Software Design Description

- DMS Test P7an

- DMS Test Procedures

\subsection{RESPONSIBILITIES}

\subsection{DMS DEVELOPERS}

The DMS developers, under the direction of the BCSR Project Manager, are responsible for providing all applications software, including security programs, scripts, and command files. They are also responsible for software unit and functional testing prior to turn over of the fully configured system for system testing. The developers will provide input and review for test plan and test procedure development, and will be available to answer questions during test execution. The DMS developers will be responsible for software configuration management and software changes required to correct test discrepancies. 
WHC-SD-W026-TP-010, Rev. 0

\subsection{DMS TEST TEAMS}

The WRAP Project DMS Test Team will be responsible for overall execution and management of the formal DMS acceptance testing, including test plan development, test procedure development, scheduling, execution, and documenting each test. The WRAP Project test team is under the direction of the WRAP Project cognizant engineer.

The BCSR DMS Test Team will be responsible for execution and management of shop and site dry run testing, including test plan review, test procedure review, scheduling, execution, and documenting each test. The BCSR test team is under the direction of the BCSR DMS Project Manager.

\subsection{QUALITY ASSURANCE}

Quality Assurance will approve the Test Plan.

\subsection{STAFFING AND TRAINING}

\section{I TEST CASE PREPARATION}

If the SQA TeamTest software is used, two individuals with training on the SQA TeamTest software will be required to prepare automated test scripts.

\subsection{SYSTEM OPERATION AND MAINTENANCE}

HP system administration support, ORACLE system administration support, LAN administration support, and DMS development team support will be required to establish the test environment and to respond to operational problems during testing.

\subsection{TEST EXECUTION}

A test director, test operators, and test witness wil1 be required for test execution. The test operators must be trained in use of the DMS and the SQA TeamTest software, if used.

\subsection{SCHEDULE}

Test Plan Approved

System Test Procedures Complete

Unit Testing Complete

Shop Testing on Deveiopment System Complete

Software Installed on Production Hardware

* Shop Test Report Issued

* Site Acceptance Testing Complete

* Final Test Report Issued
9/ $1 / 95$

2/ $1 / 96$

$2 / 16 / 96$

$2 / 23 / 96$

3/ $1 / 96$

4) $1 / 96$

6/ 1/96

7/ $1 / 96$

* BCSR support not provided for these activities under Phase 1 funding. 
WHC-SD-W026-TP-010, Rev. 0

\subsection{RISKS AND CONTINGENCIES}

Any delay in installation of the DMS computer system in WRAP 1 and turnover of the system for software testing will impact the acceptance testing schedule and could potentially impact the WRAP operational testing and plant startup.

\subsection{REFERENCES}

BNFL, 1994, Waste Receiving and Processing Facility Drum NDA Integrated Assemb7y - Interface Definition Document, Ro, British Nuclear Fuels Ltd., Sellafjeld, Seascale, Cumbria, United Kingdom.

IEEE, 1983 (Reaffirmed 1991), IEEE Standard for Software Test Documentation, ANSI/IEEE Standard 829-1983, IEEE, New York, New York.

IEEE, 1989, IEEE Standard Glossary of Sof tware Engineering Techno7ogy, ANSI/IEEE Standard 729-1983, New York, New York.

SII, 1994, Waste Receiving and Processing (WRAP) Facility Plant Management System - Network Communications Protoco 7 Definition Document, Rev. 2.1, Systems Interface, Inc., Bothel1, Washington.

WHC, 1990, Quality Assurance Manual, WHC-CM-4-2, Westinghouse Hanford Company, Richland, Washington.

WHC, 1994a, Waste Receiving and Processing Facility Module 1 Computer Interface Definition Document, WHC-SD-W026-CSWD-001, Rev. 0, Westinghouse Hanford Company, Richland, Washington.

WHC, 1994b, Waste Receiving and Processing Facility Module 1 Data Management System Software Project Management Plan, WHC-SD-W026-SDP-001, Rev. 0, Westinghouse Hanford Company, Richland, Washington.

WHC, 1994c, Waste Receiving and Processing Facility Module 1 Data Management System Software Requirements Specification, WHC-SD-W026-CSRS-001, Rev. 0, Westinghouse Hanford Company, Richland, Washington.

WHC, 1995, Waste Receiving and Processing Facility Module 1 Data Management System Software Design Description, WHC-SO-W026-SDD-001, Rev. 1, Westinghouse Hanford Company, Richland, Washington.

\subsection{TRADEMARKS}

A. Oracle is a trademark of Oracle Corporation, World Headquarters, 500 Oracle Parkway, Redwood Shores, CA 94063

B. TeamTest is a trademark of Software QuaTity Automation, Inc., 10 State Street, Woburn, MA 01801 
WHC-SD-W026-TP-010, Rev, 0

\subsection{GLOSSARY}

Acceptance Testing. Formal testing conducted to determine whether or not a system satisfies its acceptance criteria and to enable the customer to determine whether or not to accept the system. (IEEE 1012-1986)

Execution Number. A sequential number signifying the number of times a test procedure has been executed. The first time a test procedure is used the execution number is one.

Functional Testing. Testing conducted to evaluate the compliance of a system or component with specified functional requirements. (IEEE 610.12-1990)

Interface Testing. Testing conducted to evaluate whether systems or components pass data and control correctly to one another. (IEEE $610.12-1990)$

Integration Testing. Testing in which software components, hardware components, or both are combined and tested to evaluate the interaction between them. (IEEE 610.12-1990)

Pass/Fail Criteria. Decision rules used to determine whether a software item or a software feature passes or fails a test. (IEEE 829-1983)

Regression Testing. Selective retesting of a system or component to verify that modifications have not caused unintended effects and that the system or component still complies with its specified requirements. (IEEE 610.12-1990)

Software Item. Source code, object code, job control code, control data, or a collection of these items. (IEEE 829-1983)

System Testing. Testing conducted on a complete, integrated system to evaluate the system's compliance with its specified requirements. 610.12-1990)

Test (Procedure) Discrepancy. An occurrence where actual output differs substantialiy from expected output, resulting in test failure.

Test Case. Documentation specifying inputs, predicted results, and a set of execution conditions for a test item. (IEEE 829-1983)

Test Director. The person designated to facilitate and manage testing.

Test Item. A software item which is an object of testing. (IEEE 829-1983)

Test Log. A chronological record of relevant details about the execution of tests. (IEEE 610.12-1990)

Test operator. The person designated to prepare equipment for testing and perform Test Procedures. 


$$
\text { WHC-SD-W026-TP-010, Rev. } 0
$$

Test Plan. A document describing the scope, approach, resources, and schedule of intended testing activities. It jdentifies test items, the features to be tested, the testing tasks, who will do each task, and any risks requiring contingency planning. (IEEE 829-1983)

Test Procedure. Documentation specifying a sequence of actions for the execution of a test. (IEEE 829-1983)

Test Report. A document that describes the conduct and results of the testing carried out for a system or component. (IEEE 610.12-1990)

Test Witness. The person designated to witness testing. The witness verifies that each test procedure step is correctiy executed and that the output received from each step matches the output expected from each step. Multiple test witnesses may participate in testing.

Unit Testing. Testing of individuat hardware or software units or groups of related units. (IEEE 610.12-1990) 
WHC-SD-W026-TP-010, Rev. 0

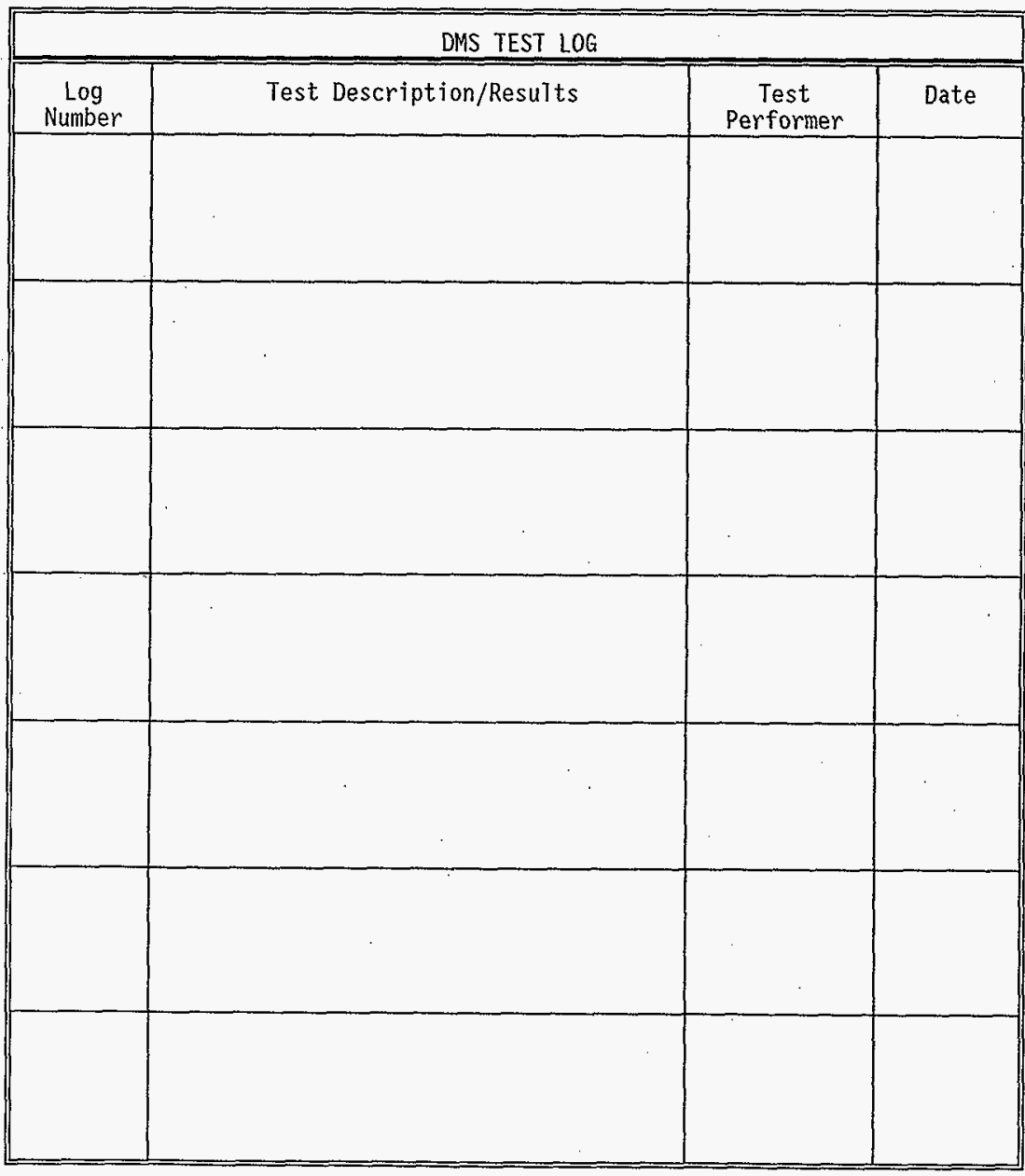

Figure 1. Example DMS Unit and FunctionaT Test Log 
WHC-SD-W026-TP-010, Rev. 0

\section{EXAMPLE TEST PROCEDURE COVER SHEET}

Test Procedure Name:

Test Procedure ID:

Revision Number:

Execution Number:

Start Date-Time:

Test Operator:

End Date-Time:

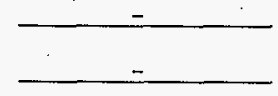

Witness:

\section{Test Exceptions Recorded?}

$\square$ Yes

$\square$ No

\section{COMMENTS}

\section{TEST PROCEDURE APPROVAL}

TEST $\square$ Successfu] $\square$ UnsuccessfuT

BCSR Representative:

Date:

WHC Projects Representative:

Date:

WHC NFS Representative:

Date:

(NFS = New Facility Startup)

Figure 2. Example Test Procedure Cover Page 
WHC-SD-W026-TP-010, Rev. 0

\section{EXAMPLE TEST PROCEDURE DISCREPANCY REPORT}

Test Procedure Name:

Test Procedure ID:

Revision \#:

Procedure Step \#:

Execution:

Test Operator:

Date:

Witness:

Date:

Expected Result

Actual Result

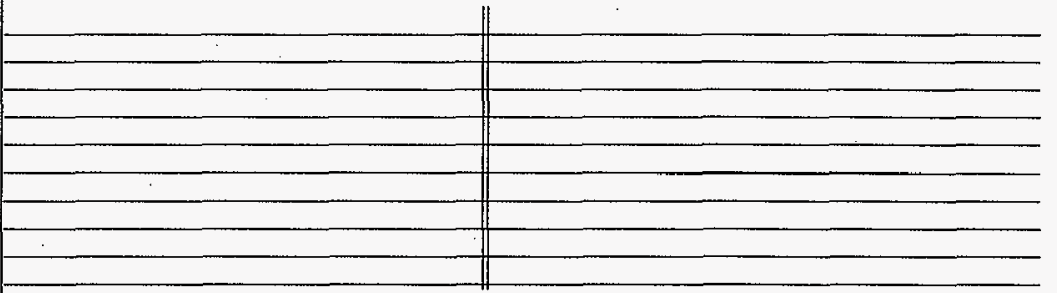

RESOLUTION:

Date Resolved:

APPROVAL OF RESOLUTION

BCSR Representative:

WHC Projects Representative:

Date:

WHC NFS Representative:

Date:

Date:

Figure 3. Example Test Procedure Discrepancy Report 


\begin{tabular}{|c|c|c|}
\hline 13 WRAP W WI & 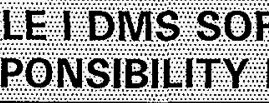 & $\mathrm{MABE} / \mathrm{FESTNG}$ \\
\hline 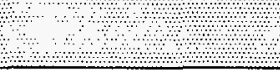 & , S $^{\text {SHOP IESTING }}$ & S \\
\hline & $\begin{array}{c}\text { BCSR Shop Testing } \\
\text { (Dry Run) }\end{array}$ & WRAP Project Shop Testing \\
\hline Test Director & & \\
\hline Test Operator(s) & & \\
\hline Test Witness & & \\
\hline Quality Assurance & & \\
\hline & SIIE IESTING & \\
\hline & $\begin{array}{c}\text { BCSR Site Testing } \\
\text { (Dry Run) }\end{array}$ & WRAP Project Sịte Testing \\
\hline Test Director & & \\
\hline Test Operator(s) & & \\
\hline Test Witness & & \\
\hline Quality Assurance & & \\
\hline WHC Project Witness & & \\
\hline WHC N.F.S. Witness & & \\
\hline
\end{tabular}

Figure 4. Testing responsibility Matrix

${ }^{1}$ BCSR support of DMS Site Testing is not provided under Phase 1 funding. 
HNF-SD-WM-TRP-287, Rev. 0

\section{APPENDIX B \\ Functional Test Results}




\begin{tabular}{|c|c|c|c|c|c|c|c|c|c|}
\hline PROC \# & $\begin{array}{l}\text { PROCEDURE } \\
\text { NAME }\end{array}$ & $\underset{\#}{\text { CASE }}$ & $\begin{array}{c}\text { STE } \\
\mathrm{P} \\
\# \\
\end{array}$ & STEP NAME & $\underset{\#}{\mathrm{REV}}$ & $\begin{array}{l}\text { PASS } \\
1 \text { st } \\
\text { TEST }\end{array}$ & $\begin{array}{c}\text { PASS } \\
\text { RETEST }\end{array}$ & COMMENTS & $\begin{array}{l}\text { SIGNOFF } \\
\text { DATE }\end{array}$ \\
\hline \multirow[t]{11}{*}{ DMS-F01 } & \multirow{11}{*}{$\begin{array}{l}\text { DMS/PCS Communication Interface and } \\
\text { Associated DMSCOM Processing for } \\
\text { DMSPCS, PCSDMS, and PCSRDMS } \\
\text { Messages }\end{array}$} & \multirow[t]{7}{*}{01} & 1 & $\begin{array}{l}\text { Run script folu to load data into the database. } \\
\text { Using the PCS simulator, transmit the } \\
\text { F01.01-1 message as defined in Appendix B }\end{array}$ & 0 & $\mathrm{x}$ & & & $3 / 4 / 96$ \\
\hline & & & 2 & $\begin{array}{l}\text { Using the PCS simulator, transmit the } \\
\text { F01.01-2 message as defined in Appendix B }\end{array}$ & 1 & & $\mathrm{x}$ & $\begin{array}{l}\text { requirement deleted } \\
\text { from SDD - step } \\
\text { not used }\end{array}$ & $3 / 21 / 96$ \\
\hline & & & 3 & $\begin{array}{l}\text { Using the PCS simulator, transmit the } \\
\text { F01.01-3 message as defined in Appendix B }\end{array}$ & 0 & $\mathrm{x}$ & & & $3 / 4 / 96$ \\
\hline & & & 4 & $\begin{array}{l}\text { Using the PCS simulator, transmit the } \\
\text { F01.01-4 message as defined in Appendix B }\end{array}$ & 0 & $\mathrm{x}$ & & & $3 / 4 / 96$ \\
\hline & & & 5 & Shut down the Oracle software. & 2 & & $\mathrm{x}$ & & $6 / 3 / 96$ \\
\hline & & & 6 & Bring Oracle back up. & 0 & $\mathrm{x}$ & & & $3 / 4 / 96$ \\
\hline & & & 7 & $\begin{array}{l}\text { Using the PCS simulator, transmit the } \\
\text { F01.01-6 message as defined in Appendix B }\end{array}$ & 0 & $\mathrm{x}$ & & . & $3 / 4 / 96$ \\
\hline & & \multirow[t]{3}{*}{02} & 1 . & $\begin{array}{l}\text { Using the PCS simulator, transmit the } \\
\text { F01.02-1 message as defined in Appendix B }\end{array}$ & 0 & $\mathrm{x}$ & & & $3 / 4 / 96$ \\
\hline & & & 2 & $\begin{array}{l}\text { Using the PCS simulator, transmit the } \\
\text { F01.02-2 message as defined in Appendix B }\end{array}$ & 0 & $\mathrm{x}$ & & & $3 / 4 / 96$ \\
\hline & & & 3 & $\begin{array}{l}\text { Using the PCS simulator, transmit the } \\
\text { F01.02-3 message as defined in Appendix B }\end{array}$ & 0 & $\mathrm{x}$ & & & $3 / 4 / 96$ \\
\hline & & 03 & 1 & $\begin{array}{l}\text { Using the PCS simulator, transmit the } \\
\text { F01.03-1 message as defined in Appendix B }\end{array}$ & 0 & $\mathrm{x}$ & & & $3 / 4 / 96$ \\
\hline
\end{tabular}




\begin{tabular}{|c|c|c|c|c|c|c|c|c|c|}
\hline PROC \# & $\begin{array}{l}\text { PROCEDURE } \\
\text { NAME }\end{array}$ & $\underset{\#}{\text { CASE }}$ & $\begin{array}{l}\text { STE } \\
\mathbf{P} \\
\# \\
\end{array}$ & STEP NAME & $\underset{\#}{\text { REV }}$ & $\begin{array}{l}\text { PASS } \\
\text { 1st } \\
\text { TEST }\end{array}$ & $\begin{array}{c}\text { PASS } \\
\text { RETEST }\end{array}$ & COMMENTS & $\begin{array}{l}\text { SIGNOFF } \\
\text { DATE }\end{array}$ \\
\hline \multirow[t]{11}{*}{ DMS-F01 } & \multirow{11}{*}{$\begin{array}{l}\text { DMS/PCS Communication Interface and } \\
\text { Associated DMSCOM Processing for } \\
\text { DMSPCS, PCSDMS, and PCSRDMS } \\
\text { Messages }\end{array}$} & \multirow[t]{11}{*}{03} & 2 & $\begin{array}{l}\text { Using the PCS simulator, transmit the } \\
\text { F01.03-2 message as defined in Appendix B }\end{array}$ & 0 & $x$ & & & $3 / 4 / 96$ \\
\hline & & & 3 & $\begin{array}{l}\text { Using the PCS simulator, transmit the two } \\
\text { F01.03-3 messages as defined in Appendix B }\end{array}$ & 1 & & $\mathrm{x}$ & 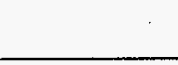 & $3 / 21 / 96$ \\
\hline & & & 4 & $\begin{array}{l}\text { Using the PCS simulator, transmit the } \\
\text { F01.03-4 message as defined in Appendix B }\end{array}$ & 1 & & $\mathrm{x}$ & $\begin{array}{l}\text { dependent on } \\
\text { F01-03-3 }\end{array}$ & $3 / 21 / 96$ \\
\hline & & & 5 & $\begin{array}{l}\text { Using the PCS simulator, transmit the } \\
\text { F01.03-5 message as defined in Appendix B }\end{array}$ & 1 & & $x$ & $\begin{array}{l}\text { dependent on } \\
\text { F01-03-3 }\end{array}$ & $3 / 21 / 96$ \\
\hline & & & 6 & $\begin{array}{l}\text { Using the PCS simulator, transmit the } \\
\text { F01.03-6 message as defined in Appendix B }\end{array}$ & 1 & & $\mathrm{x}$ & & $3 / 21 / 96$ \\
\hline & & & 7 & $\begin{array}{l}\text { Using the PCS simulator, transmit the } \\
\text { F01.03-7 message as defined in Appendix B }\end{array}$ & 2 & & $\mathrm{x}$ & & $6 / 3 / 96$ \\
\hline & & & 8 & $\begin{array}{l}\text { Using the PCS simulator, transmit the } \\
\text { F01.03-8 message as defined in Appendix B }\end{array}$ & 2 & & $x$ & & $6 / 3 / 9.6$ \\
\hline & & & 9 & $\begin{array}{l}\text { Using the PCS simulator, transmit the } \\
\text { F01.03-9 message as defined in Appendix B }\end{array}$ & 0 & $\mathrm{x}$ & & & $3 / 4 / 96$ \\
\hline & & & 10 & $\begin{array}{l}\text { Using the PCS simulator, transmit the } \\
\text { F01.03-10 message as defined in Appendix B }\end{array}$ & 0 & $\mathrm{x}$ & & & $3 / 4 / 96$ \\
\hline & & & 11 & $\begin{array}{l}\text { Using the PCS simulator, transmit the } \\
\text { F01.03-11 message as defined in Appendix B }\end{array}$ & 1 & & $\mathrm{x}$ & test script revised & $3 / 21 / 96$ \\
\hline & & & 12 & $\begin{array}{l}\text { Using the PCS simulator, transmit the } \\
\text { F01.03-12 message as defined in Appendix B }\end{array}$ & 0 & $x$ & & & $3 / 4 / 96$ \\
\hline
\end{tabular}




\begin{tabular}{|c|c|c|c|c|c|c|c|c|c|}
\hline PROC \# & $\begin{array}{l}\text { PROCEDURE } \\
\text { NAME }\end{array}$ & $\begin{array}{c}\text { CASE } \\
\#\end{array}$ & $\begin{array}{c}\text { STE } \\
\mathbf{P} \\
\#\end{array}$ & STEP NAME & $\begin{array}{c}\text { REV } \\
\#\end{array}$ & $\begin{array}{c}\text { PASS } \\
\text { 1st } \\
\text { TEST }\end{array}$ & $\begin{array}{l}\text { PASS } \\
\text { RETEST }\end{array}$ & COMMIENTS & $\begin{array}{l}\text { SIGNOFF } \\
\text { DATE }\end{array}$ \\
\hline \multirow[t]{11}{*}{ DMS-F01 } & \multirow{11}{*}{$\begin{array}{l}\text { DMS/PCS Communication Interface and } \\
\text { Associated DMSCOM Processing for } \\
\text { DMSPCS, PCSDMS, and PCSRDMS } \\
\text { Messages }\end{array}$} & \multirow[t]{11}{*}{04} & 1 & $\begin{array}{l}\text { Using the PCS simulator, transmit the } \\
\text { F01.04-1 message as defined in Appendix B }\end{array}$ & 1 & & $\mathrm{x}$ & & $3 / 21 / 96$ \\
\hline & & & 2 & $\begin{array}{l}\text { Using the PCS simulator, transmit the } \\
\text { F01.04-2 message as defined in Appendix B }\end{array}$ & 1 & & $\dot{x}$ & & $3 / 21 / 96$ \\
\hline & & & 3 & $\begin{array}{l}\text { Using the PCS simulator, transmit the } \\
\text { F01.04-3 message as defined in Appendix B }\end{array}$ & 1 & & $\mathrm{X}$ & & $3 / 21 / 96$ \\
\hline & & & 4 & $\begin{array}{l}\text { Using the PCS simulator, transmit the } \\
\text { F01.04-4 message as defined in Appendix B }\end{array}$ & 1 & & $\mathrm{X}$ & & $3 / 21 / 96$ \\
\hline & & & 5 & $\begin{array}{l}\text { Using the PCS simulator, transmit the } \\
\text { F01.04-5 message as defined in Appendix B }\end{array}$ & 1 & & $\mathrm{X}$ & & $3 / 21 / 96$ \\
\hline & & & 6 & $\begin{array}{l}\text { Using the PCS simulator, transmit the } \\
\text { F01.04-6 message as defined in Appendix B }\end{array}$ & 1 & & $\mathrm{X}$ & . & $3 / 21 / 96$ \\
\hline & & & 7 & $\begin{array}{l}\text { Using the PCS simulator, transmit the } \\
\text { F01.04-7 message as defined in Appendix B }\end{array}$ & 1 & & $\mathrm{x}$ & . & $3 / 21 / 96$ \\
\hline & & & 8 & $\begin{array}{l}\text { Using the PCS simulator, transmit the } \\
\text { F01.04-8 message as defined in Appendix B }\end{array}$ & 0 & $X$ & & & $3 / 4 / 96$ \\
\hline & & & 9 & $\begin{array}{l}\text { Using the PCS simulator, transmit the } \\
\text { F01.04-9 message as defined in Appendix B }\end{array}$ & 2 & & $\mathbf{x}$ & & $6 / 3 / 96$ \\
\hline & & & 10 & $\begin{array}{l}\text { Using the PCS simulator, transmit the } \\
\text { F01.04-10 messages as defined in Appendix B }\end{array}$ & 2 & & $\mathrm{X}$ & & $6 / 3 / 96$ \\
\hline & & & 11 & $\begin{array}{l}\text { Using the PCS simulator, transmit the } \\
\text { F01.04-11 message as defined in Appendix B }\end{array}$ & 2 & & $\mathrm{X}$ & & $6 / 3 / 96$ \\
\hline
\end{tabular}




\begin{tabular}{|c|c|c|c|c|c|c|c|c|c|}
\hline PROC $\not$ & $\begin{array}{l}\text { PROCEDURE } \\
\text { NAME }\end{array}$ & $\underset{\#}{\text { CASE }}$ & $\begin{array}{c}\text { STE } \\
\mathbf{p} \\
\#\end{array}$ & STEP NAME & $\underset{\#}{\text { REV }}$ & $\begin{array}{c}\text { PASS } \\
\text { 1st } \\
\text { TEST }\end{array}$ & $\begin{array}{l}\text { PASS } \\
\text { RETEST }\end{array}$ & COMMENTS & $\begin{array}{l}\text { SIGNOFF } \\
\text { DATE }\end{array}$ \\
\hline \multirow[t]{9}{*}{ DMS-F01 } & \multirow{9}{*}{$\begin{array}{l}\text { DMS/PCS Communication Interface and } \\
\text { Associated DMSCOM Processing for } \\
\text { DMSPCS, PCSDMS, and PCSRDMS } \\
\text { Messages }\end{array}$} & 04 & 12 & $\begin{array}{l}\text { Using the PCS simulator, transmit the } \\
\text { F01.04-12 message as defined in Appendix B }\end{array}$ & 2 & & $\mathrm{x}$ & & $6 / 3 / 96$ \\
\hline & & \multirow[t]{8}{*}{05} & 1 & $\begin{array}{l}\text { Using the PCS simulator, transmit the } \\
\text { F01.05-1 messages as defined in Appendix B }\end{array}$ & 2 & & $x$ & & $6 / 3 / 96$ \\
\hline & & & 2 & $\begin{array}{l}\text { Using the PCS simulator, transmit the } \\
\text { F01.05-2 messages as defined in Appendix B }\end{array}$ & 2 & & $\mathrm{x}$ & & $6 / 3 / 96$ \\
\hline & & & 3 & $\begin{array}{l}\text { Using the PCS simulator, transmit the } \\
\text { F01.05-3 messages as defined in Appendix B }\end{array}$ & 2 & & $x$ & & $3 / 29 / 96$ \\
\hline & & & 4 & $\begin{array}{l}\text { Using the PCS simulator, transmit the } \\
\text { F01.05-4 message as defined in Appendix B }\end{array}$ & 0 & $\mathrm{x}$ & & & $3 / 4 / 96$ \\
\hline & & & 5 & $\begin{array}{l}\text { Using the PCS simulator, transmit the } \\
\text { F01.05-5 message as defined in Appendix B }\end{array}$ & 0 & $\mathrm{x}$ & & & $3 / 4 / 96$ \\
\hline & & & 6 & $\begin{array}{l}\text { Using the PCS simulator, transmit the } \\
\text { F01.05-6 message as defined in Appendix B }\end{array}$ & 0 & $x$ & & & $3 / 4 / 96$ \\
\hline & & & 7 & $\begin{array}{l}\text { Using the PCS simulator, transmit the } \\
\text { F01.05-7 message as defined in Appendix B }\end{array}$ & 0 & $\mathrm{x}$ & & & $3 / 4 / 96$ \\
\hline & & & 8 & $\begin{array}{l}\text { Provide multiple messages to DMSCOM using } \\
\text { both the PCS and StE simulator (sce F01-05-8 } \\
\text { in Appendix B). }\end{array}$ & 2 & & $\mathrm{x}$ & & $6 / 3 / 96$ \\
\hline DMS-F04 & $\begin{array}{l}\text { DMS/SIE Communication Interface and } \\
\text { Associated DMSCOM Processing for } \\
\text { DMSSIE DD, DMSRSIE, and SIEDMS } \\
\text { Messages }\end{array}$ & 01 & 1 & $\begin{array}{l}\text { Using the PCS simulator, transmit the } \\
\text { F04.01-1 message as defined in Appendix B }\end{array}$ & 1 & & $\mathrm{x}$ & & $4 / 23 / 96$ \\
\hline
\end{tabular}




\begin{tabular}{|c|c|c|c|c|c|c|c|c|c|}
\hline PROC \# & $\begin{array}{l}\text { PROCEDURE } \\
\text { NAME }\end{array}$ & $\begin{array}{c}\text { CASE } \\
\#\end{array}$ & $\begin{array}{c}\text { STE } \\
\mathbf{P} \\
\#\end{array}$ & STEP NAME & $\underset{\#}{\text { REV }}$ & $\begin{array}{c}\text { PASS } \\
\text { Ist } \\
\text { TEST }\end{array}$ & $\begin{array}{c}\text { PASS } \\
\text { RETEST }\end{array}$ & COMMENTS & $\begin{array}{l}\text { SIGNOFF } \\
\text { DATE }\end{array}$ \\
\hline \multirow[t]{3}{*}{ DMS-F04 } & \multirow{8}{*}{$\begin{array}{l}\text { DMS/SIE Communication Interface and } \\
\text { Associated DMSCOM Processing for } \\
\text { DMSSIE DD, DMSRSIE, and SIEDMS } \\
\text { Messages }\end{array}$} & \multirow[t]{7}{*}{01} & 2 & $\begin{array}{l}\text { Using the PCS simulator, transmit the } \\
\text { F04.01-2 message as defined in Appendix B }\end{array}$ & 1 & & $\mathrm{X}$ & & $4 / 23 / 96$ \\
\hline & & & 3 & $\begin{array}{l}\text { Using the PCS simulator, transmit the } \\
\text { F04.01-3 message for drum TST-F04-WV-13A } \\
\text { as defined in Appendix.B }\end{array}$ & 1 & & $X$ & & $4 / 23 / 96$ \\
\hline & & & 4 & $\begin{array}{l}\text { Using the PCS simulator, transmit the } \\
\text { F04.01-4 message for drum TST-F04-WV-13B } \\
\text { as defined in Appendix B }\end{array}$ & 1 & & $\mathrm{x}$ & & $4 / 23 / 96$ \\
\hline \multirow{5}{*}{. } & & & 5 & $\begin{array}{l}\text { Using the PCS simulator, transmit the } \\
\text { F04.01-5 message as defined in Appendix B to } \\
\text { test the transmittal of SIE data for } 55 \text {-gallon } \\
\text { waste drums overpacked in } 85-\mathrm{g}\end{array}$ & 1 & & $\mathrm{X}$ & & $4 / 23 / 96$ \\
\hline & & & 6 & $\begin{array}{l}\text { Using the PCS simulator, transmit the } \\
\text { F04.01-6 message as defined in Appendix B to } \\
\text { test the transmittal of SIE data for 55-gallon } \\
\text { waste drums which are not over }\end{array}$ & 1 & & $\mathrm{x}$ & & $4 / 23 / 96$ \\
\hline & & & 7 & $\begin{array}{l}\text { Using the PCS simulator, transmit the } \\
\text { F04.01-7 message as defined in Appendix B }\end{array}$ & 1 & & $\mathrm{X}$ & . & $4 / 23 / 96$ \\
\hline & & & 8 & $\begin{array}{l}\text { Using the PCS simulator, transmit the } \\
\text { F04.01-8 message as defined in Appendix B }\end{array}$ & 1 & & $\mathrm{X}$ & & $4 / 23 / 96$ \\
\hline & & 02 & 1 & $\begin{array}{l}\text { Using the PCS simulator, transmit the } \\
\text { F04.02-1 message as defined in Appendix B }\end{array}$ & 0 & $\mathrm{X}$ & & & $3 / 20 / 96$ \\
\hline
\end{tabular}




\begin{tabular}{|c|c|c|c|c|c|c|c|c|c|}
\hline PROC \# & $\begin{array}{l}\text { PROCEDURE } \\
\text { NAME }\end{array}$ & $\begin{array}{c}\text { CASE } \\
\#\end{array}$ & $\begin{array}{c}\text { STE } \\
\mathbf{P} \\
\# \\
\end{array}$ & STEP NAME & $\underset{\#}{\mathrm{REV}}$ & $\begin{array}{l}\text { PASS } \\
\text { Ist } \\
\text { TEST }\end{array}$ & $\begin{array}{c}\text { PASS } \\
\text { RETEST }\end{array}$ & COMMENTS & $\begin{array}{l}\text { SIGNOFF } \\
\text { DATE }\end{array}$ \\
\hline \multirow[t]{8}{*}{ DMS-F04 } & \multirow{8}{*}{$\begin{array}{l}\text { DMS/SIE Communication Interface and } \\
\text { Associated DMSCOM Processing for } \\
\text { DMSSIE DD, DMSRSIE, and SIEDMS } \\
\text { Messages }\end{array}$} & \multirow[t]{3}{*}{02} & 2 & $\begin{array}{l}\text { Using the PCS simulator, transmit the } \\
\text { F04.02-1 message as defined in Appendix B }\end{array}$ & 0 & $\mathrm{x}$ & & & $3 / 20 / 96$ \\
\hline & & & 3 & $\begin{array}{l}\text { Using the PCS simulator, transmit the } \\
\text { F04.02-1 message as defined in Appendix B }\end{array}$ & 0 & $\mathrm{x}$ & & & $3 / 20 / 96$ \\
\hline & & & $\dot{4}$ & $\begin{array}{l}\text { Using the PCS simulator, transmit the } \\
\text { F04.02-1 message as defined in Appendix B }\end{array}$ & 0 & $\mathrm{x}$ & & & $3 / 20 / 96$ \\
\hline & & \multirow[t]{5}{*}{03} & 1 & $\begin{array}{l}\text { Using the SIE simulator, transmit the F04:03-1 } \\
\text { message as defined in Appendix B to check for } \\
\text { criticality message transmittal }\end{array}$ & 0 & $\mathrm{x}$ & . & & $3 / 20 / 96$ \\
\hline & & & 2 & $\begin{array}{l}\text { Using the SIE simulator, transmit the F04.03-2 } \\
\text { message as defined in Appendix B to verify } \\
\text { SIE status messaging functionality }\end{array}$ & 0 & $\mathrm{x}$ & & & $3 / 20 / 96$ \\
\hline & & & 3 & $\begin{array}{l}\text { Using the SIE simulator, transmit the F04.03-3 } \\
\text { message as defined in Appendix B to simulate } \\
\text { the transmittal of LLW assay results from the } \\
\text { SIE }\end{array}$ & 1 & & $\mathrm{x}$ & & $4 / 23 / 96$ \\
\hline & & & 4 & $\begin{array}{l}\text { Using the SIE simulator, transmit the F04.03-4 } \\
\text { message as defined in Appendix B to simulate } \\
\text { the transmittal of TRU assay results from the } \\
\text { SIE }\end{array}$ & 1 & & $\mathrm{x}$ & & $4 / 23 / 96$ \\
\hline & & & 5 & $\begin{array}{l}\text { Using the SIE simulator, transmit the } \\
\text { F04.03-05 message as defined in Appendix B }\end{array}$ & 1 & & $x$ & & $4 / 23 / 96$ \\
\hline \multirow[t]{2}{*}{ DMS-F11 } & \multirow[t]{2}{*}{ User Logon and Main Menu Screens } & \multirow[t]{2}{*}{01} & 1 & Display the Oracle Logon window. & 0 & $\mathrm{x}$ & & & $2 / 27 / 96$ \\
\hline & & & 2 & $\begin{array}{l}\text { Logon to DMS system as a user with test role } \\
\text { UPDATE privileges. }\end{array}$ & 0 & $\mathrm{x}$ & & & $2 / 27 / 96$ \\
\hline
\end{tabular}




\begin{tabular}{|c|c|c|c|c|c|c|c|c|c|}
\hline PROC \# & $\begin{array}{l}\text { PROCEDURE } \\
\text { NAME }\end{array}$ & $\underset{\#}{\mathrm{CASE}}$ & $\begin{array}{l}\text { STE } \\
\mathbf{P} \\
\# \\
\end{array}$ & STEP NAME & $\underset{\#}{\text { REV }}$ & $\begin{array}{c}\text { PASS } \\
\text { 1st } \\
\text { TEST }\end{array}$ & $\begin{array}{c}\text { PASS } \\
\text { RETEST }\end{array}$ & COMMENTS & $\begin{array}{l}\text { SIGNOFF } \\
\text { DATE }\end{array}$ \\
\hline \multirow[t]{2}{*}{ DMS-F11 } & \multirow[t]{2}{*}{ User Logon and Main Menu Screens } & \multirow[t]{2}{*}{01} & 3 & $\begin{array}{l}\text { Verify that the proper screen is displayed with } \\
\text { the proper attributes. }\end{array}$ & 0 & $\dot{x}$ & & & $2 / 27 / 96$ \\
\hline & & & 4 & Exit the DMS system. & 0 & $\mathrm{x}$ & & & $2 / 27 / 96$ \\
\hline \multirow[t]{10}{*}{ DMS-F12 } & \multirow[t]{10}{*}{ Container Receiving screen (DMSSO101) } & \multirow[t]{10}{*}{01} & 1 & $\begin{array}{l}\text { Logon to DMS system as a user (USER1) with } \\
\text { test role UPDATE privileges. }\end{array}$ & 0 & $\mathrm{x}$ & & & $4 / 3 / 96$ \\
\hline & & & 2 & $\begin{array}{l}\text { Enter the Container Receiving screen } \\
\text { (DMSSO101) and perform the following tests. }\end{array}$ & 0 & & $\mathrm{x}$ & $\begin{array}{l}\text { WHC revised test } \\
\text { proc to match } \\
\text { approved design }\end{array}$ & $4 / 4 / 96$ \\
\hline & & & 3 & $\begin{array}{l}\text { Test the [Insert], [Commit], and [Delete] } \\
\text { button operability and Outer PIN, Inner PIN, } \\
\text { Error Status, and Signature Password fields. }\end{array}$ & 1 & & $\mathrm{x}$ & & $4 / 19 / 96$ \\
\hline & & & 4 & $\begin{array}{l}\text { Test wrong Signature Password and [Exit]. } \\
\text { button. }\end{array}$ & 0 & $x$ & & & $4 / 3 / 96$ \\
\hline & & & 5 & $\begin{array}{l}\text { Test [Next Receipt], [Previous Receipt], and } \\
\text { [Select Receipt] buttons and "Receipt \#" field. }\end{array}$ & 0 & $\mathrm{x}$ & & & $4 / 3 / 96$ \\
\hline & & & 6 & Test the $[<],[>],[<<]$, and $[>>]$ buttons. & 0 & $x$ & & & $4 / 3 / 96$ \\
\hline & & & 7 & Test the [Confirm Data] button. & 2 & & $x$ & - & $4 / 22 / 96$ \\
\hline & & & 8 & $\begin{array}{l}\text { Test the Rad Inv Alarm check when [Confirm } \\
\text { Data] button is used. }\end{array}$ & 2 & & $x$ & & $4 / 22 / 96$ \\
\hline & & & 9 & Test the [Request Cert Data] button. & 2 & & $x$ & & $4 / 22 / 96$ \\
\hline & & & 10 & $\begin{array}{l}\text { Test the functionality of "Labels" selection on } \\
\text { DMSS0101 menu. }\end{array}$ & 0 & $x$ & & & $4 / 3 / 96$ \\
\hline
\end{tabular}


DMS PHASE 1 TEST REPORT

\begin{tabular}{|c|c|c|c|c|c|c|c|c|c|}
\hline PROC \# & $\begin{array}{l}\text { PROCEDURE } \\
\text { NAME }\end{array}$ & CASE & $\begin{array}{c}\text { STE } \\
\mathbf{P} \\
\# \\
\end{array}$ & STEP NAME & $\underset{\#}{\text { REV }}$ & $\begin{array}{c}\text { PASS } \\
\text { 1st } \\
\text { TEST }\end{array}$ & $\begin{array}{c}\text { PASS } \\
\text { RETEST }\end{array}$ & COMMENTS & $\begin{array}{l}\text { SIGNOFF } \\
\text { DATE }\end{array}$ \\
\hline \multirow[t]{2}{*}{ DMS-F12 } & \multirow[t]{2}{*}{ Container Receiving screen (DMSS0101) } & \multirow[t]{2}{*}{01} & 11 & Exit the screen and the DMS system. & 0 & $x$ & & & $4 / 3 / 96$ \\
\hline & & & 12 & Exit the screen and the DMS system. & 0 & $\mathrm{x}$ & & & $4 / 3 / 96$ \\
\hline \multirow[t]{5}{*}{ DMS-F13 } & \multirow[t]{5}{*}{ Bar Code Generator screen (DMSS0102) } & \multirow[t]{5}{*}{01} & 1 & $\begin{array}{l}\text { Logon to DMS system as a user (USER1) with } \\
\text { test role UPDATE privileges. }\end{array}$ & 0 & $\mathrm{x}$ & & & $3 / 26 / 96$ \\
\hline & & & 2 & $\begin{array}{l}\text { Enter the DMSS0102 Bar Code Generator } \\
\text { screen and perform the following tests. }\end{array}$ & 0 & $\mathrm{x}$ & & & $3 / 26 / 96$ \\
\hline & & & 3 & Print a barcode & 0 & & $\mathrm{x}$ & & $8 / 9 / 96$ \\
\hline & & & 4 & Test the [EXIT] button. & 0 & $\mathrm{x}$ & & & $3 / 26 / 96$ \\
\hline & & & 5 & Exit the DMS system. & 0 & $\mathrm{x}$ & & & $3 / 26 / 96$ \\
\hline \multirow[t]{7}{*}{ DMS-F14 } & \multirow[t]{7}{*}{ NDE screen (DMSS0201) } & \multirow[t]{7}{*}{01} & 1 & Load Data Script F14.01-01U. & 0 & $\mathrm{x}$ & & & $5 / 23 / 96$ \\
\hline & & & 2 & $\begin{array}{l}\text { Logon to DMS system as a user (USER1) with } \\
\text { test role UPDATE privileges. }\end{array}$ & 0 & $\mathrm{x}$ & & & $5 / 23 / 96$ \\
\hline & & & 3 & $\begin{array}{l}\text { Select the NDE screen (DMSS0201) from the } \\
\text { DMS Main Menu: }\end{array}$ & 0 & $\mathrm{x}$ & & & $5 / 23 / 96$ \\
\hline & & & 4 & $\begin{array}{l}\text { Test NDE Results PIN field for required or } \\
\text { optional entry: }\end{array}$ & 0 & $\mathrm{x}$ & & & $5 / 23 / 96$ \\
\hline & & & 5 & $\begin{array}{l}\text { Test NDE Results PIN field for updatability, } \\
\text { case restriction, format, length, and valid } \\
\text { value: }\end{array}$ & 1 & & $\mathrm{x}$ & & $6 / 20 / 96$ \\
\hline & & & 6 & $\begin{array}{l}\text { Enter NDE Results PIN which has previous } \\
\text { NDE results: }\end{array}$ & 0 & $\mathrm{x}$ & & & $5 / 23 / 96$ \\
\hline & & & 7 & Tesst $[<]$ and $[>]$ buttons for entered PIN: & 0 & $\mathrm{x}$ & & & $5 / 23 / 96$ \\
\hline
\end{tabular}




\begin{tabular}{|c|c|c|c|c|c|c|c|c|c|}
\hline PROC \# & $\begin{array}{l}\text { PROCEDURE } \\
\text { NAME }\end{array}$ & CASE & $\begin{array}{c}\text { STE } \\
\mathbf{P} \\
\#\end{array}$ & STEP NAME & REV & $\begin{array}{c}\text { PASS } \\
\text { 1st } \\
\text { TEST }\end{array}$ & $\begin{array}{l}\text { PASS } \\
\text { RETEST }\end{array}$ & COMMENTS & $\begin{array}{l}\text { SIGNOFF } \\
\text { DATE }\end{array}$ \\
\hline \multirow[t]{12}{*}{ DMS-F14 } & \multirow[t]{12}{*}{ NDE sereen (DMSS0201) } & \multirow[t]{12}{*}{01} & 8 & $\begin{array}{l}\text { Enter another NDE Results PIN which has } \\
\text { previous NDE results: }\end{array}$ & 0 & $\mathrm{x}$ & & & $5 / 23 / 96$ \\
\hline & & & 9 & $\begin{array}{l}\text { Test [Exit] button for prompt to commit } \\
\text { changes: }\end{array}$ & 0 & $\mathrm{x}$ & & & $5 / 23 / 96$ \\
\hline & & & 10 & $\begin{array}{l}\text { Test [Waste Record] button; no NDE Results } \\
\text { PIN displayed: }\end{array}$ & 0 & $\mathrm{x}$ & & $\therefore$ & $5 / 23 / 96$ \\
\hline & & & 11 & $\begin{array}{l}\text { Test [PhysComp] button; no NDE Results PIN } \\
\text { displayed: }\end{array}$ & 0 & $\mathrm{X}$ & & & $5 / 23 / 96$ \\
\hline & & & 12 & $\begin{array}{l}\text { Test [Rad Detail] button; no NDE Results PIN } \\
\text { displayed: }\end{array}$ & 0 & $\mathrm{x}$ & & & $5 / 23 / 96$ \\
\hline & & & 13 & Select Previous NDE Results Drum PIN: & 0 & $\mathrm{x}$ & & & $5 / 23 / 96$ \\
\hline & & & 14 & $\begin{array}{l}\text { Test }[<<] \text { and }[>>] \text { buttons for selected } \\
\text { PIN: }\end{array}$ & 0 & $\mathrm{x}$ & & . & $5 / 23 / 96$ \\
\hline & & & 16 & $\begin{array}{l}\text { Test up and down arrow keys to view records } \\
\text { for selected PIN (F14-TST-WP-005): }\end{array}$ & 0 & $\mathrm{x}$ & & & $5 / 23 / 96$ \\
\hline & & & 17 & $\begin{array}{l}\text { Select another drum PIN from View Previous } \\
\text { NDE Results list: }\end{array}$ & 0 & $\mathrm{x}$ & & & $5 / 23 / 96$ \\
\hline & & & 18 & Test [Commit] button: & 0 & $\mathrm{x}$ & & & $5 / 23 / 96$ \\
\hline & & & 19 & Load Data Script F14.01-19U & 0 & $\mathrm{x}$ & & & $5 / 23 / 96$ \\
\hline & & & 20 & $\begin{array}{l}\text { DMSCOM CL message received - Outer Drum } \\
\text { PIN 'TST-FI4-OD-002' at Vault B: }\end{array}$ & 0 & $\mathrm{x}$ & & & $5 / 23 / 96$ \\
\hline
\end{tabular}




\begin{tabular}{|c|c|c|c|c|c|c|c|c|c|}
\hline PROC \# & $\begin{array}{l}\text { PROCEDURE } \\
\text { NAME }\end{array}$ & $\underset{\#}{\mathrm{CASE}}$ & $\begin{array}{c}\text { STE } \\
\mathbf{P} \\
\#\end{array}$ & STEP NAME & $\underset{\#}{\text { REV }}$ & $\begin{array}{l}\text { PASS } \\
\text { 1st } \\
\text { TEST }\end{array}$ & $\begin{array}{c}\text { PASS } \\
\text { RETEST }\end{array}$ & COMMENTS & $\begin{array}{l}\text { SIGNOFF } \\
\text { DATE }\end{array}$ \\
\hline \multirow[t]{12}{*}{ DMS-F14 } & \multirow[t]{12}{*}{ NDE screen (DMSS0201) } & \multirow[t]{12}{*}{01} & 21. & $\begin{array}{l}\text { Reselect PIN 'TST-F14-PD-002' from View } \\
\text { Previous NDE Results list: }\end{array}$ & 1 & & OPEN & & \\
\hline & & & 22 & $\begin{array}{l}\text { Test [Waste Record] button for selected PIN } \\
\text { (F14-TST-PD-002): }\end{array}$ & 1 & & OPEN & $\begin{array}{l}\text { Dependent on } \\
\text { F14.01-21 }\end{array}$ & \\
\hline & & & $22 \mathrm{~A}$ & Test screen for record scrolling: & 1 & & $\mathrm{x}$ & & $6 / 20 / 96$ \\
\hline & & & $22 \mathrm{~B}$ & Test delete record function: & 1 & & $\mathrm{x}$ & & $6 / 20 / 96$ \\
\hline & & & $22 \mathrm{C}$ & $\begin{array}{l}\text { Test Primary Waste Type field for Autohint } \\
\text { and updatability: }\end{array}$ & 1 & . & $x$ & & $6 / 20 / 96$ \\
\hline & & & $22 \mathrm{D}$ & $\begin{array}{l}\text { Test Chemical Nature field for Autohint and } \\
\text { updatability: }\end{array}$ & 1 & & $x$ & & $6 / 20 / 96$ \\
\hline & & & $22 \mathrm{E}$ & $\begin{array}{l}\text { Test Storage Category field for Autohint and } \\
\text { updatability: }\end{array}$ & 1 & & $\mathrm{x}$ & & $6 / 20 / 96$ \\
\hline & & & $22 \mathrm{~F}$ & $\begin{array}{l}\text { Test Primary Waste Type field for Autohint } \\
\text { and updatability: }\end{array}$ & 1 & & $\mathrm{x}$ & & $6 / 20 / 96$ \\
\hline & & & $22 \mathrm{G}$ & $\begin{array}{l}\text { Test Primary Waste Type field for Autohint } \\
\text { and updatability: }\end{array}$ & 1 & & $\mathrm{x}$ & & $6 / 20 / 96$ \\
\hline & & & $22 \mathrm{H}$ & $\begin{array}{l}\text { Test Routine field for Autohint and } \\
\text { updatability: }\end{array}$ & 1 & & $\mathrm{x}$ & & $6 / 20 / 96$ \\
\hline & & & $22 I$ & $\begin{array}{l}\text { Test Secondary PIN field for Autohint and } \\
\text { updatability: }\end{array}$ & 1 & . & $\mathrm{x}$ & & $6 / 20 / 96$ \\
\hline & & & $22 J$ & $\begin{array}{l}\text { Test Gross Weight field for Autohint and } \\
\text { updatability: }\end{array}$ & 1 & & $\mathrm{x}$ & & $8 / 20 / 96$ \\
\hline
\end{tabular}




\begin{tabular}{|c|c|c|c|c|c|c|c|c|c|}
\hline PROC \# & $\begin{array}{l}\text { PROCEDURE } \\
\text { NAME }\end{array}$ & $\underset{\#}{\text { CASE }}$ & $\begin{array}{c}\text { STE } \\
\mathbf{P} \\
\#\end{array}$ & STEP NAME & $\underset{\#}{\operatorname{REV}}$ & $\begin{array}{l}\text { PASS } \\
\text { 1st } \\
\text { TEST }\end{array}$ & $\begin{array}{c}\text { PASS } \\
\text { RETEST }\end{array}$ & COMMENTS & $\begin{array}{l}\text { SIGNOFF } \\
\text { DATE }\end{array}$ \\
\hline \multirow[t]{12}{*}{ DMS-F14 } & \multirow[t]{12}{*}{ NDE screen (DMSS0201) } & \multirow[t]{12}{*}{01 . } & $22 \mathrm{~K}$ & $\begin{array}{l}\text { Test Waste Weight field for Autohint and } \\
\text { updatability: }\end{array}$ & 1 & & $\mathrm{x}$ & & $8 / 20 / 96$ \\
\hline & & & $22 \mathrm{~L}$ & $\begin{array}{l}\text { Test Generator Comment field for Autohint } \\
\text { and updatability: }\end{array}$ & 1 & & $\mathrm{x}$ & & $6 / 20 / 96$ \\
\hline & & & $22 \mathrm{M}$ & $\begin{array}{l}\text { Test Waste Description field for Autohint and } \\
\text { updatability: }\end{array}$ & 1 & & $\mathrm{x}$ & . & $6 / 20 / 96$ \\
\hline & & & $22 \mathrm{~N}$ & $\begin{array}{l}\text { Test Accumulation Date field for Autohint and } \\
\text { updatability: }\end{array}$ & 1 & & $\mathrm{x}$ & & $6 / 20 / 96$ \\
\hline & & & 220 & $\begin{array}{l}\text { Test Package Date field for Autohint and } \\
\text { updatability: }\end{array}$ & 1 & & $\mathrm{x}$ & & $6 / 20 / 96$ \\
\hline & & & $22 \mathrm{P}$ & $\begin{array}{l}\text { Test Treatment Date field for Autohint and } \\
\text { updatability: }\end{array}$ & 1 & & $\mathrm{x}$ & . & $6 / 20 / 96$ \\
\hline & & & $22 \mathrm{Q}$ & $\begin{array}{l}\text { Test TSD Accept Date field for Autohint and } \\
\text { updatability: }\end{array}$ & 1 & & $\mathrm{x}$ & & $6 / 20 / 96$ \\
\hline & & & $22 R$ & $\begin{array}{l}\text { Test Source Cmpny Id field for Autohint and } \\
\text { updatability: }\end{array}$ & 1 & & $\mathrm{x}$ & & $6 / 20 / 96$ \\
\hline & & & $22 S$ & $\begin{array}{l}\text { Test Source Facil Id field for Autohint and } \\
\text { updatability: }\end{array}$ & 1. & & $\mathrm{x}$ & . & $6 / 20 / 96$ \\
\hline & & & 23 & Test [Return to Main 201 Screen] button: & 0 & $\mathrm{x}$ & & & $5 / 23 / 96$ \\
\hline & & & 24 & $\begin{array}{l}\text { Test [PhysComp] button for selected PIN } \\
\text { (TST-F14-PD-002): }\end{array}$ & 0 & $\mathrm{x}$ & & & $5 / 23 / 96$ \\
\hline & & & $25 \mathrm{~A}$ & Test screen for record navigation: & 0 & $x$ & & & $5 / 23 / 96$ \\
\hline
\end{tabular}




\begin{tabular}{|c|c|c|c|c|c|c|c|c|c|}
\hline PROC \# & $\begin{array}{l}\text { PROCEDURE } \\
\text { NAME }\end{array}$ & $\underset{\#}{\mathrm{CASE}}$ & $\begin{array}{l}\text { STE } \\
\mathbf{P} \\
\# \\
\end{array}$ & STEP NAME & $\underset{\#}{\text { REV }}$ & $\begin{array}{l}\text { PASS } \\
1 \text { st } \\
\text { TEST }\end{array}$ & $\begin{array}{c}\text { PASS } \\
\text { RETEST }\end{array}$ & COMMENTS & $\begin{array}{l}\text { SIGNOFF } \\
\text { DATE }\end{array}$ \\
\hline \multirow[t]{13}{*}{ DMS-F14 } & \multirow[t]{13}{*}{ NDE screen (DMSS0201) } & \multirow[t]{13}{*}{01} & $25 \mathrm{~B}$ & Test delete record function: & 0 & $\mathrm{x}$ & & & $5 / 23 / 96$ \\
\hline & & & $25 \mathrm{C}$ & Test Description field for updatability: & 0 & $\mathrm{x}$ & & & $5 / 23 / 96$ \\
\hline & & & $25 \mathrm{D}$ & $\begin{array}{l}\text { Test Volume Percent field for Autohint and } \\
\text { updatability: }\end{array}$ & 0 & $\mathrm{x}$ & & & $5 / 23 / 96$ \\
\hline & & & 26 & Test [Return to Main 201 Screen] button: & 0 & $\mathrm{x}$ & & & $5 / 23 / 96$ \\
\hline & & & 27 & $\begin{array}{l}\text { Test [Rad Detail] button for selected (previous } \\
\text { record) PIN: }\end{array}$ & 1 & & OPEN & $\begin{array}{l}\text { dependent on } \\
\text { F14.01-21 }\end{array}$ & \\
\hline & & & $28 \mathrm{~A}$ & Test screen for record scrolling: & 0 & $\mathrm{x}$ & & & $5 / 23 / 96$ \\
\hline & & & $28 \mathrm{~B}$ & Test delete record function: & 0 & $\mathrm{x}$ & & & $5 / 23 / 96$ \\
\hline & & & $28 \mathrm{C}$ & $\begin{array}{l}\text { Test Secondary Waste Type field for Autohint } \\
\text { and updatability: }\end{array}$ & 1 & & $\mathrm{x}$ & & $6 / 20 / 96$ \\
\hline & & & $28 D$ & $\begin{array}{l}\text { Test Sec. Waste Type Group field for Autohint } \\
\text { and updatability: }\end{array}$ & 1 & & $x$ & & $6 / 20 / 96$ \\
\hline & & & $28 \mathrm{E}$ & $\begin{array}{l}\text { Test Content Thermal Poweer field for } \\
\text { Autohint and updatability: }\end{array}$ & 1 & & $\mathrm{x}$ & & $6 / 20 / 96$ \\
\hline & & & $28 \mathrm{~F}$ & $\begin{array}{l}\text { Test Seal Number field for Autohint and } \\
\text { updatability: }\end{array}$ & 1 & & $\mathrm{x}$ & & $6 / 20 / 96$ \\
\hline & & & $28 G$ & $\begin{array}{l}\text { Test Assay Number field for Autohint and } \\
\text { updatability: }\end{array}$ & 1 & & $\mathrm{x}$ & & $6 / 20 / 96$ \\
\hline & & & $28 \mathrm{H}$ & $\begin{array}{l}\text { Test Assay Date field for Autohint and } \\
\text { updatability: }\end{array}$ & 1 & & $\mathrm{x}$ & & $6 / 20 / 96$ \\
\hline
\end{tabular}




\begin{tabular}{|c|c|c|c|c|c|c|c|c|c|}
\hline PROC $\#$ & $\begin{array}{l}\text { PROCEDURE } \\
\text { NAME }\end{array}$ & $\underset{\#}{\text { CASE }}$ & $\begin{array}{c}\text { STE } \\
\mathrm{P} \\
\# \\
\end{array}$ & STEP. NAME & $\underset{\#}{\mathrm{REV}}$ & $\begin{array}{c}\text { PASS } \\
\text { 1st } \\
\text { TEST } \\
\end{array}$ & $\begin{array}{c}\text { PASS } \\
\text { RETEST }\end{array}$ & COMMENTS & $\begin{array}{l}\text { SIGNOFF } \\
\text { DATE }\end{array}$ \\
\hline \multirow[t]{11}{*}{ DMS-F14 } & \multirow[t]{11}{*}{ NDE screen (DMSS0201) } & \multirow[t]{11}{*}{01} & $28 !$ & $\begin{array}{l}\text { Test Waste Category field for Autohint and } \\
\text { updatability: }\end{array}$ & 1 & & $\mathrm{x}$ & & $6 / 20 / 96$ \\
\hline & & & $28 \mathrm{~J}$ & $\begin{array}{l}\text { Test Waste Makeup field for Autohint and } \\
\text { updatability: }\end{array}$ & 1 & & $\mathrm{x}$ & & $6 / 20 / 96$ \\
\hline & & & $28 \mathrm{~K}$ & $\begin{array}{l}\text { Test Contact/Remote Handling field for } \\
\text { Autohing and updatability: }\end{array}$ & 1 & & $\mathrm{x}$ & & $6 / 20 / 96$ \\
\hline & & & $28 \mathrm{~L}$ & $\begin{array}{l}\text { Test Void Space Filler field for Autohint and } \\
\text { updatability: }\end{array}$ & 1 & & $\mathrm{x}$ & - & $6 / 20 / 96$ \\
\hline & & & $28 \mathrm{M}$ & $\begin{array}{l}\text { Test SWIMS Category field for Autohint and } \\
\text { updatability: }\end{array}$ & 1 & & $\mathrm{x}$ & & $6 / 20 / 96$ \\
\hline & & & $28 \mathrm{~N}$ & $\begin{array}{l}\text { Test WRAP Category field for Autohint and } \\
\text { updatability: }\end{array}$ & 1 & & $\mathrm{x}$ & & $6 / 20 / 96$ \\
\hline & & & 280 & $\begin{array}{l}\text { Test RSWIMS Container Count field for } \\
\text { Autohint and updatability: }\end{array}$ & 1 & & $\mathrm{x}$ & & $6 / 20 / 96$ \\
\hline & & & $28 \mathrm{P}$ & $\begin{array}{l}\text { Test Container Dose Rate (mrem/hr) freld for } \\
\text { Autohint and updatability: }\end{array}$ & 2. & & $\mathrm{X}$ & & $6 / 20 / 96$ \\
\hline & & & $28 Q$ & $\begin{array}{l}\text { Test Container Neutron Dose Rate field for } \\
\text { Autohint and updatability: }\end{array}$ & 1 & & $\mathrm{x}$ & & $6 / 20 / 96$ \\
\hline & & & $28 \mathrm{R}$ & $\begin{array}{l}\text { Test Content Organic Volume \% field for } \\
\text { Autohint and updatability: }\end{array}$ & 1 & & $\mathrm{x}$ & & $6 / 20 / 96$ \\
\hline & & & $28 \mathrm{~S}$ & $\begin{array}{l}\text { Test Content Organic Weight }(\mathrm{kg}) \text { freld for } \\
\text { Autohint and updatability: }\end{array}$ & 1 & & $\mathrm{x}$ & & $6 / 20 / 96$ \\
\hline
\end{tabular}




\begin{tabular}{|c|c|c|c|c|c|c|c|c|c|}
\hline PROC \# & $\begin{array}{l}\text { PROCEDURE } \\
\text { NAME }\end{array}$ & CASE & $\begin{array}{c}\text { STE } \\
\mathbf{P} \\
\#\end{array}$ & STEP NAME & $\underset{\#}{\text { REV }}$ & $\begin{array}{l}\text { PASS } \\
\text { Ist } \\
\text { TEST }\end{array}$ & $\begin{array}{l}\text { PASS } \\
\text { RETEST }\end{array}$ & COMMENTS & $\begin{array}{l}\text { SIGNOFF } \\
\text { DATE }\end{array}$ \\
\hline \multirow[t]{13}{*}{ DMS-F14 } & \multirow[t]{13}{*}{ NDE screen (DMSS0201) } & \multirow[t]{13}{*}{01} & $28 \mathrm{~T}$ & $\begin{array}{l}\text { Test Total Alpha Curies freld for Autohint and } \\
\text { updatability: }\end{array}$ & 1 & & $\mathrm{x}$ & & $6 / 20 / 96$ \\
\hline & & & $28 \mathrm{U}$ & $\begin{array}{l}\text { Test Total Beta/Gamma Curies field for } \\
\text { Autohint and updatability: }\end{array}$ & 1 & & $\mathrm{x}$ & & $6 / 20 / 96$ \\
\hline & & & $28 \mathrm{~V}$ & $\begin{array}{l}\text { Test Total Dose Equivalent Curies field for } \\
\text { Autohint and updatability: }\end{array}$ & 1 & & $\mathrm{x}$ & & $6 / 20 / 96$ \\
\hline & & & $28 \mathrm{~W}$ & $\begin{array}{l}\text { Test Total PU Equivalent Curies field for } \\
\text { Autohint and updatability: }\end{array}$ & 1 & & $x$ & & $6 / 20 / 96$ \\
\hline & & & $28 \mathrm{x}$ & $\begin{array}{l}\text { Test Total PU Fissle Gram Equiv. field for } \\
\text { Autohint and updatability: }\end{array}$ & 1 & & $\mathrm{x}$ & & $6 / 20 / 96$ \\
\hline & & & $28 \mathrm{Y}$ & $\begin{array}{l}\text { Test Void Space Filler Descr. field for } \\
\text { Autohint and updatability: }\end{array}$ & 1 & & $\mathrm{x}$ & & $6 / 20 / 96$ \\
\hline & & & $28 Z$ & $\begin{array}{l}\text { Test SWMS Category Descr. field for } \\
\text { Autohint and updatability: }\end{array}$ & 1 & & $\mathrm{x}$ & & $6 / 20 / 96$ \\
\hline & & & $28 \mathrm{AA}$ & $\begin{array}{l}\text { Test WRAP Category Descr. field for Autohint } \\
\text { and updatability: }\end{array}$ & 1 & & $\mathrm{x}$ & & $6 / 20 / 96$ \\
\hline & & & 29 & Test [Return to Main 201 Screen] button: & 0 & $\mathrm{x}$ & & & $5 / 23 / 96$ \\
\hline & & & 30 & Test 'Box' radio button: & 0 & $\mathrm{x}$ & & & $5 / 23 / 96$ \\
\hline & & & 31 & Test [Vault B] button: & 0 & $x$ & & & $5 / 23 / 96$ \\
\hline & & & 32 & Load Data Script F14.01-32U & 0 & $\mathrm{x}$ & & & $5 / 23 / 96$ \\
\hline & & & 33 & $\begin{array}{l}\text { DMSCOM CL message received - PIN } \\
\text { 'TST-F14-BX-002' at Box NDE: }\end{array}$ & 0 & $\mathrm{x}$ & & & $5 / 23 / 96$ \\
\hline
\end{tabular}




\begin{tabular}{|c|c|c|c|c|c|c|c|c|c|}
\hline PROC \# & $\begin{array}{l}\text { PROCEDURE } \\
\text { NAME }\end{array}$ & $\underset{\#}{\text { CASE }}$ & $\begin{array}{c}\text { STE } \\
\mathbf{P} \\
\# \\
\end{array}$ & STEP NAME & $\underset{\#}{\text { REV }}$ & $\begin{array}{c}\text { PASS } \\
\text { 1st } \\
\text { TEST } \\
\end{array}$ & $\begin{array}{c}\text { PASS } \\
\text { RETEST }\end{array}$ & COMMENTS & $\begin{array}{l}\text { SIGNOFF } \\
\text { DATE }\end{array}$ \\
\hline \multirow[t]{13}{*}{ DMS-F14 } & \multirow[t]{13}{*}{ NDE screen (DMSS0201) } & \multirow[t]{13}{*}{01} & 34 & Test [Commit] button: & 1 & OPEN & & $\begin{array}{l}\text { dependent on } \\
\text { F14.01-21 }\end{array}$ & \\
\hline & & & 35 & $\begin{array}{l}\text { Test [Vault B] button (current location of PIN } \\
\text { with no previous NDE records): }\end{array}$ & 0 & $\mathrm{x}$ & & & $5 / 23 / 96$ \\
\hline & & & 36 & Test [Waste Record] button for new PIN: & 1 & & $\mathrm{x}$ & & $6 / 20 / 96$ \\
\hline & & & 37 & $\begin{array}{l}\text { Close Container and Content Record (WASTE) } \\
\text { Screen: }\end{array}$ & 0 & $\mathrm{x}$ & & & $5 / 23 / 96$ \\
\hline & & & 38 & Test [PhysComp] button for new PIN: & 0 & $\mathrm{x}$ & & & $5 / 23 / 96$ \\
\hline & & & 39 & Close Physical Component Screen: & 0 & $\mathrm{x}$ & & & $5 / 23 / 96$ \\
\hline & & & 40 & Test [Rad Detail] button for new PIN: & 1 & & $x$ & & $6 / 20 / 96$ \\
\hline & & & 41 & $\begin{array}{l}\text { Close Radioactive Waste Container Detail } \\
\text { Record Screen: }\end{array}$ & 0 & $\mathrm{x}$ & & & $5 / 23 / 96$ \\
\hline & & & 43 & Test Outer Drum PIN field for updatability: & 0 & $\mathrm{x}$ & & & $5 / 23 / 96$ \\
\hline & & & 44 & $\begin{array}{l}\text { Test Date field for updatability, format, and } \\
\text { valid value: }\end{array}$ & 1 & & $\mathrm{x}$ & & $8 / 20 / 96$ \\
\hline & & & 45 & Test Operator field for updatability: & 0 & $\mathrm{x}$ & & & $5 / 23 / 96$ \\
\hline & & & 46 & $\begin{array}{l}\text { Test updatability, case restriction, format, and } \\
\text { length of Video Disk File field: }\end{array}$ & 0 & $\mathrm{x}$ & & & $5 / 23 / 96$ \\
\hline & & & 47 & $\begin{array}{l}\text { Test updatability, case restriction, format, and } \\
\text { length of Video Tape No. field: }\end{array}$ & 0 & $\mathrm{x}$ & & & $5 / 23 / 96$ \\
\hline
\end{tabular}


DATE: 9/27/96

\begin{tabular}{|c|c|c|c|c|c|c|c|c|c|}
\hline PROC \# & $\begin{array}{l}\text { PROCEDURE } \\
\text { NAME }\end{array}$ & $\underset{\#}{\text { CASE }}$ & $\begin{array}{c}\text { STE } \\
\mathbf{P} \\
\#\end{array}$ & STEP NAME & $\underset{\#}{\mathrm{REV}}$ & $\begin{array}{c}\text { PASS } \\
\text { 1st } \\
\text { TEST }\end{array}$ & $\begin{array}{l}\text { PASS } \\
\text { RETEST }\end{array}$ & COMMENTS & $\begin{array}{l}\text { SIGNOFF } \\
\text { DATE }\end{array}$ \\
\hline \multirow[t]{14}{*}{ DMS-F14 } & \multirow[t]{14}{*}{ NDE screen (DMSS0201) } & \multirow[t]{14}{*}{01} & 48 & $\begin{array}{l}\text { Test updatability, case restriction, format, and } \\
\text { length of Video Disk No. field: }\end{array}$ & 0 & $\mathrm{x}$ & & & $5 / 23 / 96$ \\
\hline & & & 49 & $\begin{array}{l}\text { Test updatability, case restriction, format, and } \\
\text { length of Video Tape Start field: }\end{array}$ & 0 & $\mathrm{x}$ & & & $5 / 23 / 96$ \\
\hline & & & 50 & $\begin{array}{l}\text { Test updatability, case restriction, format, and } \\
\text { length of NDE Comments field: }\end{array}$ & 0 & $x$ & & & $5 / 23 / 96$ \\
\hline & & & 51 & $\begin{array}{l}\text { Test updatability, case restriction, format, and } \\
\text { length of Contents Verified field: }\end{array}$ & 1 & & $\mathrm{x}$ & & $8 / 20 / 96$ \\
\hline & & & 52 & $\begin{array}{l}\text { Test updatability, case restriction, format, and } \\
\text { length of Compliant field: }\end{array}$ & 0 & $x$ & & & $5 / 23 / 96$ \\
\hline & & & 53 & Load Data Script F14.01-53U. & 0 & $\mathrm{x}$ & & & $5 / 23 / 96$ \\
\hline & & & 54 & $\begin{array}{l}\text { DMSCOM CL messages received - PIN } \\
\text { TST-F14-WP-004 at Vault A, PIN } \\
\text { 'TST-F14-OD-002' at PAN B, PIN } \\
\text { 'TST-F14-PD-005' at Vault B: }\end{array}$ & 0 & $\mathrm{x}$ & & & $5 / 23 / 96$ \\
\hline & & & 55 & Test [Insert] button: & & & OPEN & & \\
\hline & & & 56 & Test for required data fields: & 0 & $\mathrm{x}$ & & & $5 / 23 / 96$ \\
\hline & & & 57 & Test [Commit] button: & 0 & $\mathrm{x}$ & & & $5 / 23 / 96$ \\
\hline & & & 58 & Test [Vault B] button: & 0 & $\mathrm{x}$ & & & $5 / 23 / 96$ \\
\hline & & & 59 & Insert a new record: & 0 & $\mathrm{x}$ & & & $5 / 23 / 96$ \\
\hline & & & 60 & Test Delete function for newly entered record: & 0 & $\mathrm{x}$ & & & $5 / 23 / 96$ \\
\hline & & & 61 & Test Rollback function: & 0 & $x$ & & & $5 / 23 / 96$ \\
\hline
\end{tabular}




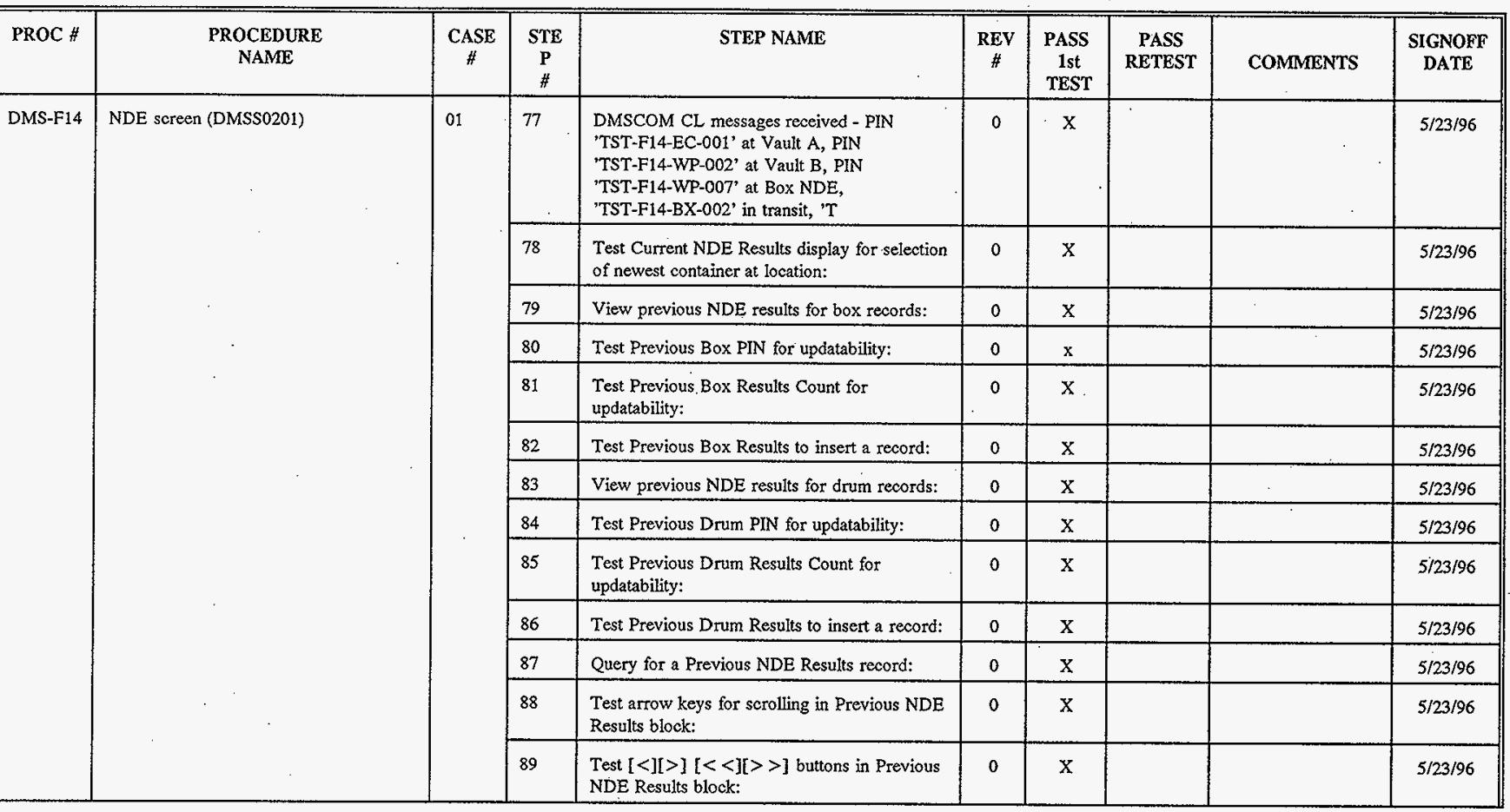




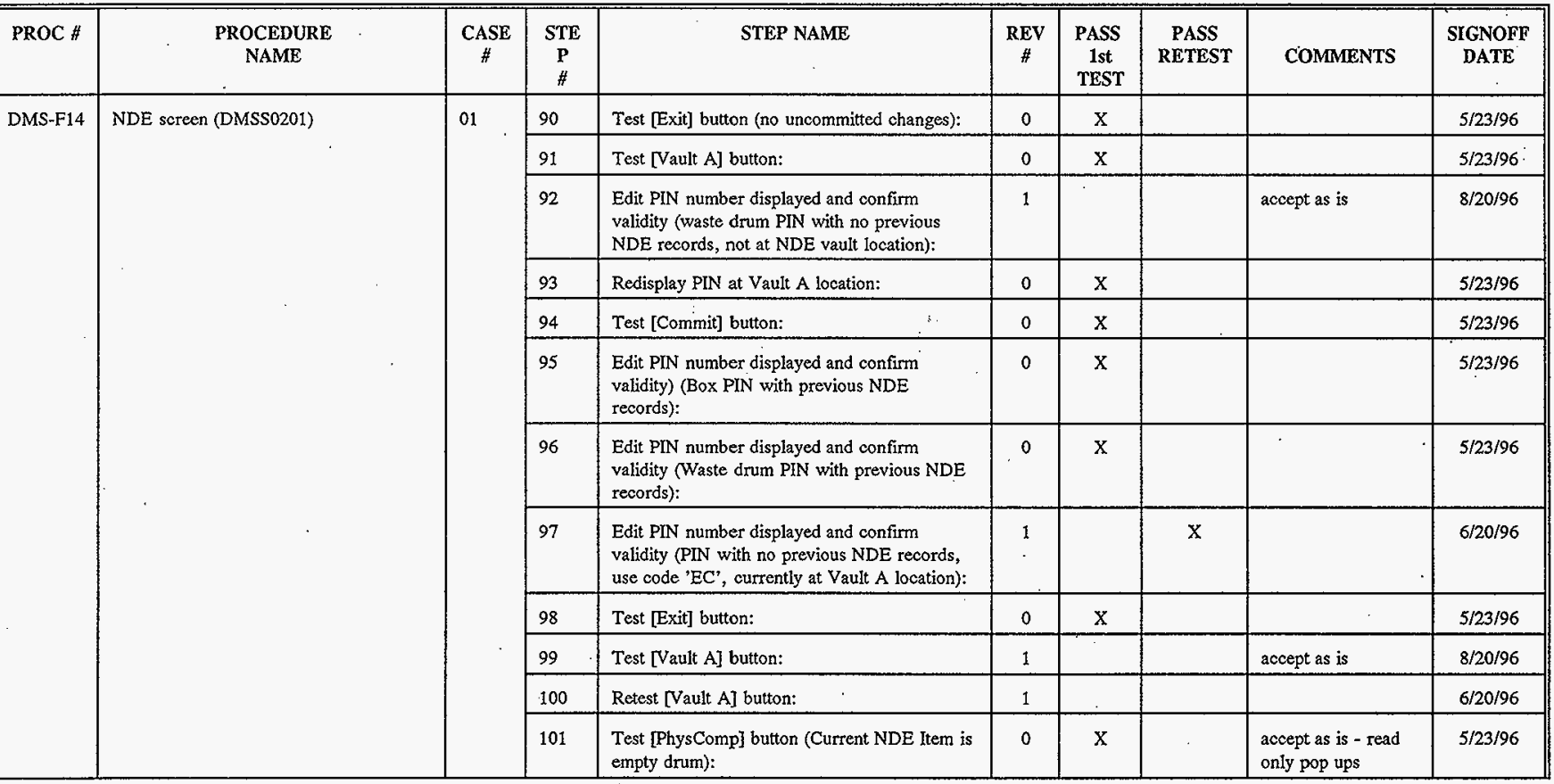




\begin{tabular}{|c|c|c|c|c|c|c|c|c|c|}
\hline PROC \# & $\begin{array}{l}\text { PROCEDURE } \\
\text { NAME }\end{array}$ & $\underset{\#}{\text { CASE }}$ & $\begin{array}{c}\text { STE } \\
P \\
\#\end{array}$ & STEP NAME & $\underset{\#}{\operatorname{REV}}$ & $\begin{array}{l}\text { PASS } \\
\text { 1st } \\
\text { TEST }\end{array}$ & $\begin{array}{c}\text { PASS } \\
\text { RETEST }\end{array}$ & COMMENTS & $\begin{array}{l}\text { SIGNOFF } \\
\text { DATE }\end{array}$ \\
\hline \multirow[t]{13}{*}{ DMS-F14 } & \multirow[t]{13}{*}{ NDE screen (DMSS0201) } & \multirow[t]{9}{*}{01} & 102 & $\begin{array}{l}\text { Test [Rad Detail] button (Current NDE ftem is } \\
\text { empty drum): }\end{array}$ & 0 & $\mathrm{x}$ & & $\begin{array}{l}\text { accept as is - read } \\
\text { only pop ups }\end{array}$ & $5 / 23 / 96$ \\
\hline & & & 103 & Load Data Script F14.01-103U & 0 & $\mathrm{x}$ & & & $5 / 23 / 96$ \\
\hline & & & 104 & $\begin{array}{l}\text { DMSCOM CL messages received - PIN } \\
\text { 'TST-F14-EC-001' at Empty Container } \\
\text { Storage, PIN 'TST-F14-WP-002' at AS/RS: }\end{array}$ & 1 & & $\mathrm{x}$ & $\begin{array}{l}\text { test data specs and } \\
\text { test script revised }\end{array}$ & $8 / 26 / 96$ \\
\hline & & & 105 & Test [Box] button: & 0 & $\mathrm{x}$ & & & $5 / 23 / 96$ \\
\hline & & & 106 & $\begin{array}{l}\text { Confirm validity of current PIN (drum PIN at } \\
\text { Box location): }\end{array}$ & 0 & $\mathrm{x}$ & & & $5 / 23 / 96$ \\
\hline & & & 107 & Test [Exit] button: & 0 & $x$ & & & $5 / 23 / 96$ \\
\hline & & & $108^{\circ}$ & Exit the DMS system. & 0 & $\mathrm{x}$ & & & $5 / 23 / 96$ \\
\hline & & & 109 & Perform post-test database query 'f14.01q a'. & 0 & $\mathrm{x}$ & & & $5 / 23 / 96$ \\
\hline & & & 110 & $\begin{array}{l}\text { Close test database, DMSCOM, and PCS } \\
\text { simulator Dynacomm instances. }\end{array}$ & 0 & $\mathbf{x}$ & & & $5 / 23 / 96$ \\
\hline & & \multirow[t]{4}{*}{02} & 1 & Load Data Script F14.02-01U. & 1 & $\mathrm{x}$ & & & $9 / 4 / 96$ \\
\hline & & & 2 & $\begin{array}{l}\text { Logon to DMS system as a user (USER1) with } \\
\text { test role UPDATE privileges. }\end{array}$ & 1 & $\mathrm{x}$ & & & $9 / 4 / 96$ \\
\hline & & & 3 & $\begin{array}{l}\text { Select the NDE screen (DMSS0201) from the } \\
\text { DMS Main Menu: }\end{array}$ & 1 & $\mathrm{x}$ & & & $9 / 4 / 96$ \\
\hline & & & 4 & $\begin{array}{l}\text { Test NDE Results PIN field for required or } \\
\text { optional entry: }\end{array}$ & 1 & $x$ & & & $9 / 4 / 96$ \\
\hline
\end{tabular}




\begin{tabular}{|c|c|c|c|c|c|c|c|c|c|}
\hline PROC \# & $\begin{array}{l}\text { PROCEDURE } \\
\text { NAME }\end{array}$ & $\underset{\#}{\text { CASE }}$ & $\begin{array}{c}\text { STE } \\
\mathbf{P} \\
\#\end{array}$ & STEP NAME & $\underset{\#}{\text { REV }}$ & $\begin{array}{l}\text { PASS } \\
\text { 1st } \\
\text { TEST }\end{array}$ & $\begin{array}{c}\text { PASS } \\
\text { RETEST }\end{array}$ & COMMENTS & $\begin{array}{l}\text { SIGNOFF } \\
\text { DATE }\end{array}$ \\
\hline \multirow[t]{12}{*}{ DMS-F14 } & \multirow[t]{12}{*}{ NDE screen (DMSS0201) } & \multirow[t]{12}{*}{02} & 5 & $\begin{array}{l}\text { Test NDE Results PIN field for updatability, } \\
\text { case restriction, format, length, and valid } \\
\text { value: }\end{array}$ & 1 & $\mathrm{x}$ & & & $9 / 4 / 96$ \\
\hline & & & 6 & $\begin{array}{l}\text { Enter NDE Results PIN which has previous } \\
\text { NDE results: }\end{array}$ & 1 & $\mathrm{x}$ & & & $9 / 4 / 96$ \\
\hline & & & 7 & Test $[<]$ and $[>]$ buttons for entered PIN: & 1 & $\mathrm{x}$ & & & 9/4/96 \\
\hline & & & 8 & $\begin{array}{l}\text { Enter another NDE Results PIN which has } \\
\text { previous NDE results: }\end{array}$ & 1 & $\mathrm{x}$ & & & $9 / 4 / 96$ \\
\hline & & & 9 & $\begin{array}{l}\text { Test [Exit] button for prompt to commit } \\
\text { changes: }\end{array}$ & & & OPEN & & \\
\hline & & & 10 & $\begin{array}{l}\text { Test [Waste Record] button; no NDE Results } \\
\text { PIN displayed: }\end{array}$ & 1 & $\mathrm{x}$ & & & $9 / 4 / 96$ \\
\hline & & & 11 & $\begin{array}{l}\text { Test [PhysComp] button; no NDE Results PIN } \\
\text { displayed: }\end{array}$ & 1 & $\mathrm{x}$ & & & $9 / 4 / 96$ \\
\hline & & & 12 & $\begin{array}{l}\text { Test [Rad Detail] button; no NDE Results PIN } \\
\text { displayed: }\end{array}$ & 1 & $\mathrm{x}$ & & & $9 / 4 / 96$ \\
\hline & & & 13 & Select Previous NDE Results Drum PIN: & 1 & $x$ & & & 9/4/96 \\
\hline & & & 14 & $\begin{array}{l}\text { Test }[<<] \text { and }[>>] \text { buttons for selected } \\
\text { PIN: }\end{array}$ & 1 & $\mathrm{x}$ & & & $9 / 4 / 96$ \\
\hline & & & 15 & Enter new record for selected PIN: & 1 & $\mathbf{x}$ & & & $9 / 4 / 96$ \\
\hline & & & 16 & $\begin{array}{l}\text { Test up and down arrow keys to view records } \\
\text { for selected PIN (F14-TST-WP-005): }\end{array}$ & 1 & $\mathrm{x}$ & & & 9/4/96 \\
\hline
\end{tabular}




\begin{tabular}{|c|c|c|c|c|c|c|c|c|c|}
\hline PROC \# & $\begin{array}{l}\text { PROCEDURE } \\
\text { NAME }\end{array}$ & $\underset{\#}{\text { CASE }}$ & $\begin{array}{c}\text { STE } \\
\mathrm{P} \\
\# \\
\end{array}$ & STEP NAME & $\underset{\#}{\text { REV }}$ & $\begin{array}{c}\text { PASS } \\
\text { 1st } \\
\text { TEST }\end{array}$ & $\begin{array}{c}\text { PASS } \\
\text { RETEST }\end{array}$ & COMMENTS & $\begin{array}{c}\text { SIGNOFF } \\
\text { DATE }\end{array}$ \\
\hline \multirow[t]{12}{*}{ DMS-F14 } & \multirow[t]{12}{*}{ NDE screen (DMSS0201) } & \multirow[t]{12}{*}{02} & 17 & $\begin{array}{l}\text { Select another drum PIN from View Previous } \\
\text { NDE Results list: }\end{array}$ & 1 & $\mathrm{x}$ & & & $9 / 4 / 96$ \\
\hline & & & 18 & Test [Commit] button: & 1 & $\mathrm{x}$ & & & $9 / 4 / 96$ \\
\hline & & & 19 & Load Data Script F14.02-19U & 1 & $\mathrm{x}$ & & & $9 / 4 / 96$ \\
\hline & & & 20 & $\begin{array}{l}\text { DMSCOM CL message received - Outer Drum } \\
\text { PIN 'TST-F14-OD-002' at Vault B. }\end{array}$ & 1 & $\mathrm{x}$ & & & $9 / 4 / 96$ \\
\hline & & & 21 & $\begin{array}{l}\text { Reselect PIN 'TST-F14-PD-002' from View } \\
\text { Previous NDE Results list: }\end{array}$ & & & OPEN & same as F14-01.21 & \\
\hline & & & 22 & $\begin{array}{l}\text { Test [Waste Record] button for selected PIN } \\
\text { (F14-TST-PD-002): }\end{array}$ & 1 & & OPEN & $\begin{array}{l}\text { dep on F14-01.21 } \\
\text { same as F14-01.22 }\end{array}$ & \\
\hline & & & $22 \mathrm{~A}$ & Test screen for record scrolling: & 1 & $\mathrm{x}$ & & & $9 / 4 / 96$ \\
\hline & & & $22 \mathrm{~B}$ & Test delete record function: & 1 & $x$ & & & $9 / 4 / 96$ \\
\hline & & & 22D & $\begin{array}{l}\text { Test Chemical Nature field for Autohint and } \\
\text { updatability: }\end{array}$ & 1 & $\mathrm{x}$ & & & $9 / 4 / 96$ \\
\hline & & & $22 \mathrm{E}$ & $\begin{array}{l}\text { Test Storage Category field for Autohint and } \\
\text { updatability: }\end{array}$ & 1 & $\mathrm{x}$ & & & $9 / 4 / 96$ \\
\hline & & & $22 \mathrm{~F}$ & $\begin{array}{l}\text { Test Primary Waste Type field for Autohint } \\
\text { and updatability: }\end{array}$ & 1 & $\mathrm{x}$ & & & $9 / 4 / 96$ \\
\hline & & & $22 G$ & $\begin{array}{l}\text { Test Primary Waste Type field for Autohint } \\
\text { and updatability: }\end{array}$ & 1 & $\mathrm{x}$ & & & $9 / 4 / 96$ \\
\hline
\end{tabular}




\begin{tabular}{|c|c|c|c|c|c|c|c|c|c|}
\hline PROC \# & $\begin{array}{l}\text { PROCEDURE } \\
\text { NAME }\end{array}$ & $\underset{\#}{\text { CASE }}$ & $\begin{array}{c}\text { STE } \\
\mathbf{P} \\
\#\end{array}$ & STEP NAME & $\underset{\#}{\mathrm{REV}}$ & $\begin{array}{c}\text { PASS } \\
\text { 1st } \\
\text { TEST }\end{array}$ & $\begin{array}{c}\text { PASS } \\
\text { RETEST }\end{array}$ & COMMENTS & $\begin{array}{l}\text { SIGNOFF } \\
\text { DATE }\end{array}$ \\
\hline \multirow[t]{11}{*}{ DMS-F14 } & \multirow[t]{11}{*}{ NDE screen (DMSS0201) } & \multirow[t]{11}{*}{02} & $22 \mathrm{H}$ & $\begin{array}{l}\text { Test Routine field for Autohint and } \\
\text { updatability: }\end{array}$ & 1 & $\mathrm{x}$ & & & 9/4/96 \\
\hline & & & $22 I$ & $\begin{array}{l}\text { Test Secondary PIN field for Autohint and } \\
\text { updatability: }\end{array}$ & 1 & $\mathrm{X}$ & & & $9 / 4 / 96$ \\
\hline & & & $22 \mathrm{~J}$ & $\begin{array}{l}\text { Test Gross Weight field for Autohint and } \\
\text { updatability: }\end{array}$ & 1 & $\mathrm{x}$ & & & $9 / 4 / 96$ \\
\hline & & & $22 \mathrm{~K}$ & $\begin{array}{l}\text { Test Waste Weight field for Autohint and } \\
\text { updatability: }\end{array}$ & 1 & $\mathrm{x}$. & & & $9 / 4 / 96$ \\
\hline & & & $22 \mathrm{~L}$ & $\begin{array}{l}\text { Test Generator Comment field for Autohint } \\
\text { and updatability: }\end{array}$ & 1 & $\mathrm{x}$ & & & $9 / 4 / 96$ \\
\hline & & & $22 \mathrm{M}$ & $\begin{array}{l}\text { Test Waste Description field for Autohint and } \\
\text { updatability: }\end{array}$ & 1 & $x$ & & & $9 / 4 / 96$ \\
\hline & & & $22 \mathrm{~N}$ & $\begin{array}{l}\text { Test Accumulation Date field for Autohint and } \\
\text { updatability: }\end{array}$ & 1 & $\mathrm{x}$ & & & $9 / 4 / 96$ \\
\hline & & & 220 & $\begin{array}{l}\text { Test Package Date field for Autohint and } \\
\text { updatability: }\end{array}$ & 1 & $x$ & & & $9 / 4 / 96$ \\
\hline & & & $22 P$ & $\begin{array}{l}\text { Test Treatment Date field for Autohint and } \\
\text { updatability: }\end{array}$ & 1 & $\mathrm{x}$ & & & $9 / 4 / 96$ \\
\hline & & & $22 Q$ & $\begin{array}{l}\text { Test TSD Accept Date field for Autohint and } \\
\text { updatability: }\end{array}$ & 1 & $x$ & & . & $9 / 4 / 96$ \\
\hline & & & $22 R$ & $\begin{array}{l}\text { Test Source Cmpny Id field for Autohint and } \\
\text { updatability: }\end{array}$ & 1 & $\mathrm{x}$ & & & $9 / 4 / 96$ \\
\hline
\end{tabular}




\begin{tabular}{|c|c|c|c|c|c|c|c|c|c|}
\hline PROC \# & $\begin{array}{l}\text { PROCEDURE } \\
\text { NAME }\end{array}$ & $\underset{\#}{\mathrm{CASE}}$ & $\begin{array}{c}\text { STE } \\
\mathrm{P} \\
\# \\
\end{array}$ & STEP NAME & $\underset{\#}{\text { REV }}$ & $\begin{array}{c}\text { PASS } \\
\text { 1st } \\
\text { TEST } \\
\end{array}$ & $\begin{array}{l}\text { PASS } \\
\text { RETEST }\end{array}$ & COMMENTS & $\begin{array}{l}\text { SIGNOFF } \\
\text { DATE }\end{array}$ \\
\hline \multirow[t]{14}{*}{ DMS-F14 } & \multirow[t]{14}{*}{ NDE screen (DMSS0201) } & \multirow[t]{14}{*}{02} & $22 S$ & $\begin{array}{l}\text { Test Source Facil Id field for Autohint and } \\
\text { updatability: }\end{array}$ & 1 & $\mathrm{x}$ & & & 9/4/96 \\
\hline & & & 23 & Test [Retum to Main 201 Screen] button: & 1 & $\mathrm{x}$ & & : & $9 / 4 / 96$ \\
\hline & & & 24 & $\begin{array}{l}\text { Test [PhysComp] button for selected PIN } \\
\text { (TST-F14-PD-002): }\end{array}$ & 1 & $\mathrm{x}$ & & & $9 / 4 / 96$ \\
\hline & & & $25 \mathrm{~A}$ & Test screen for record navigation: & 1 & $x$ & & & $9 / 4 / 96$ \\
\hline & & & $25 \mathrm{~B}$ & Test delete record function: & 1 & $\mathrm{x}$ & & . & $9 / 4 / 96$ \\
\hline & & & $25 \mathrm{C}$ & Test Description field for updatability: & 1 & $\mathrm{x}$ & & & $9 / 4 / 96$ \\
\hline & & & $25 \mathrm{D}$ & $\begin{array}{l}\text { Test Volume Percent field for Autohint and } \\
\text { updatability: }\end{array}$ & 1 & $\mathrm{x}$ & & & $9 / 4 / 96$ \\
\hline & & & $25 \mathrm{E}$ & $\begin{array}{l}\text { Test Weight }(\mathrm{Kg}) \text { field for Autohint and } \\
\text { updatability: }\end{array}$ & 1 & $\mathrm{x}$ & & & $9 / 4 / 96$ \\
\hline & & & 26 & Test [Return to Main 201 Screen] button: & 1 & $\mathrm{x}$ & & & $9 / 4 / 96$ \\
\hline & & & 27 & $\begin{array}{l}\text { Test [Rad Detail] button for selected (previous } \\
\text { record) PIN: }\end{array}$ & & & OPEN & same as F14.01-27 & \\
\hline & & & $28 \mathrm{~A}$ & Test screen for record scrolling: & 1 & $\mathrm{x}$ & & & $9 / 4 / 96$ \\
\hline & & & $28 \mathrm{AA}$ & $\begin{array}{l}\text { Test WRAP Category Descr. field for Autohint } \\
\text { and updatability: }\end{array}$ & 1 & $\mathrm{x}$ & & & $9 / 4 / 96$ \\
\hline & & & $28 \mathrm{~B}$ & Test delete record function: & 1 & $\mathrm{x}$ & & & $9 / 4 / 96$ \\
\hline & & & $28 \mathrm{C}$ & $\begin{array}{l}\text { Test Secondary Waste Type field for Autohint } \\
\text { and updatability: }\end{array}$ & 1 & $x$ & & & $9 / 4 / 96$ \\
\hline
\end{tabular}




\begin{tabular}{|c|c|c|c|c|c|c|c|c|c|}
\hline PROC \# & $\begin{array}{l}\text { PROCEDURE } \\
\text { NAME }\end{array}$ & $\underset{\#}{\text { CASE }}$ & $\begin{array}{c}\text { STE } \\
\text { P } \\
\#\end{array}$ & STEP NAME & $\underset{\#}{\mathbf{R E V}}$ & $\begin{array}{c}\text { PASS } \\
\text { Ist } \\
\text { TEST }\end{array}$ & $\begin{array}{c}\text { PASS } \\
\text { RETEST }\end{array}$ & COMMENTS & $\begin{array}{l}\text { SIGNOFF } \\
\text { DATE }\end{array}$ \\
\hline \multirow[t]{11}{*}{ DMS-F14 } & \multirow[t]{11}{*}{ NDE screen (DMSS0201) } & \multirow[t]{11}{*}{02} & $28 \mathrm{D}$ & $\begin{array}{l}\text { Test Sec. Waste Type Group field for Autohint } \\
\text { and updatability: }\end{array}$ & 1 & $\mathrm{x}$ & & & $9 / 4 / 96$ \\
\hline & & & $28 \mathrm{E}$ & $\begin{array}{l}\text { Test Content Thermal Poweer field for } \\
\text { Autohint and updatability: }\end{array}$ & 1 & $\mathrm{x}$ & & & $9 / 4 / 96$ \\
\hline & & & $28 \mathrm{~F}$ & $\begin{array}{l}\text { Test Seal Number field for Autohint and } \\
\text { updatability: }\end{array}$ & .1 & $\mathrm{x}$ & & & $9 / 4 / 96$ \\
\hline & & & $28 \mathrm{G}$ & $\begin{array}{l}\text { Test Assay Number freld for Autohint and } \\
\text { updatability: }\end{array}$ & 1 & $\mathrm{x}$ & & & 9/4/96 \\
\hline & & & $28 \mathrm{H}$ & $\begin{array}{l}\text { Test Assay Date field for Autohint and } \\
\text { updatability: }\end{array}$ & 1 & $\mathrm{x}$ & & & 9/4/96 \\
\hline & & & $28 \mathrm{I}$ & $\begin{array}{l}\text { Test Waste Category field for Autohint and } \\
\text { updatability: }\end{array}$ & 1 & $\mathrm{x}$ & & & $9 / 4 / 96$ \\
\hline & & & $28 \mathrm{~J}$ & $\begin{array}{l}\text { Test Waste Makeup field for Autohint and } \\
\text { updatability: }\end{array}$ & 1 & $\mathrm{x}$ & & & $9 / 4 / 96$ \\
\hline & & & $28 \mathrm{~K}$ & $\begin{array}{l}\text { Test Contact/Remote Handling field for } \\
\text { Autohing and updatability: }\end{array}$ & 1 & $\mathrm{x}$ & & & $9 / 4 / 96$ \\
\hline & & & $28 \mathrm{~L}$ & $\begin{array}{l}\text { Test Void Space Filler field for Autohint and } \\
\text { updatability: }\end{array}$ & 1 & $\mathrm{x}$ & & & $9 / 4 / 96$ \\
\hline & & & $28 \mathrm{M}$ & $\begin{array}{l}\text { Test SWIMS Category field for Autohint and } \\
\text { updatability: }\end{array}$ & 1 & $\mathrm{x}$ & & & $9 / 4 / 96$ \\
\hline & & & $28 \mathrm{~N}$ & $\begin{array}{l}\text { Test WRAP Category field for Autohint and } \\
\text { updatability: }\end{array}$ & 1 & $\mathrm{x}$ & & & 9/4/96 \\
\hline
\end{tabular}




\begin{tabular}{|c|c|c|c|c|c|c|c|c|c|}
\hline PROC \# & $\begin{array}{l}\text { PROCEDURE } \\
\text { NAME }\end{array}$ & $\underset{\#}{\text { CASE }}$ & $\begin{array}{c}\text { STE } \\
\mathbf{P} \\
\# \\
\end{array}$ & STEP NAME & $\begin{array}{c}\text { REV } \\
\#\end{array}$ & $\begin{array}{c}\text { PASS } \\
\text { 1st } \\
\text { TEST }\end{array}$ & $\begin{array}{c}\text { PASS } \\
\text { RETEST }\end{array}$ & COMMENTS & $\begin{array}{l}\text { SIGNOFF } \\
\text { DATE }\end{array}$ \\
\hline \multirow[t]{11}{*}{ DMS-F14 } & \multirow[t]{11}{*}{ NDE screen (DMSSO201) } & \multirow[t]{11}{*}{02} & 280 & $\begin{array}{l}\text { Test RSWIMS Container Count field for } \\
\text { Autohint and updatability: . }\end{array}$ & 1 & $\mathrm{x}$ & & & $9 / 4 / 96$ \\
\hline & & & $28 \mathrm{P}$ & $\begin{array}{l}\text { Test Container Dose Rate (mrem/hr) field for } \\
\text { Autohint and updatability: }\end{array}$ & 1 & $\mathrm{x}$ & & & 9/4/96 \\
\hline & & & $28 \mathrm{Q}$ & $\begin{array}{l}\text { Test Container Neutron Dose Rate field for } \\
\text { Autohint and updatability: }\end{array}$ & 1 & $\mathrm{x}$ & & & $9 / 4 / 96$ \\
\hline & & & $28 \mathrm{R}$ & $\begin{array}{l}\text { Test Content Organic Volume \% field for } \\
\text { Autohint and updatability: }\end{array}$ & 1 & $\mathrm{x}$ & . & & $9 / 4 / 96$ \\
\hline & & & $28 S$ & $\begin{array}{l}\text { Test Content Organic Weight }(\mathrm{kg}) \text { field for } \\
\text { Autohint and updatability: }\end{array}$ & 1 & $x$ & & & $9 / 4 / 96$ \\
\hline & & & $28 \mathrm{~T}$ & $\begin{array}{l}\text { Test Total Alpha Curies field for Autohint and } \\
\text { updatability: }\end{array}$ & 1 & $\mathrm{x}$ & & & $9 / 4 / 96$ \\
\hline & & & $28 \mathrm{U}$ & $\begin{array}{l}\text { Test Total Beta/Gamma Curies field for } \\
\text { Autohint and updatability: }\end{array}$ & 1 & $\mathrm{x}$ & & & $9 / 4 / 96$ \\
\hline & & & $28 \mathrm{~V}$ & $\begin{array}{l}\text { Test Total Dose Equivalent Curies field for } \\
\text { Autohint and updatability: }\end{array}$ & 1 & $\mathrm{x}$ & & & 9/4/96 \\
\hline & & & $28 \mathrm{~W}$ & $\begin{array}{l}\text { Test Total PU Equivalent Curies field for } \\
\text { Autohint and updatability: }\end{array}$ & 1 & $\mathrm{x}$. & & & 9/4/96 \\
\hline & & & $28 \mathrm{X}$ & $\begin{array}{l}\text { Test Total PU Fissle Gram Equiv: field for } \\
\text { Autohint and updatability: }\end{array}$ & 1 & $\mathrm{x}$ & . & & 9/4/96 \\
\hline & & & $28 Y$ & $\begin{array}{l}\text { Test Void Space Filler Descr. field for } \\
\text { Autohint and updatability: }\end{array}$ & 1 & $\mathrm{x}$ & & & 9/4/96 \\
\hline
\end{tabular}




\begin{tabular}{|c|c|c|c|c|c|c|c|c|c|}
\hline PROC \# & $\begin{array}{l}\text { PROCEDURE } \\
\text { NAME }\end{array}$ & $\underset{\#}{\text { CASE }}$ & $\begin{array}{c}\text { STE } \\
\mathbf{p} \\
\# \\
\end{array}$ & STEP NAME & $\underset{\#}{\operatorname{REV}}$ & $\begin{array}{c}\text { PASS } \\
\text { 1st } \\
\text { TEST }\end{array}$ & $\begin{array}{l}\text { PASS } \\
\text { RETEST }\end{array}$ & COMMENTS & $\begin{array}{l}\text { SIGNOFF- } \\
\text { DATE }\end{array}$ \\
\hline \multirow[t]{15}{*}{ DMS-F14 } & \multirow[t]{15}{*}{ NDE screen (DMSS0201) } & \multirow[t]{15}{*}{02} & $28 \mathrm{Z}$ & $\begin{array}{l}\text { Test SWIMS Category Descr. field for } \\
\text { Autohint and updatability: }\end{array}$ & 1 & $\mathrm{x}$ & & & $9 / 4 / 96$ \\
\hline & & & 29 & Test [Return to Main 201 Screen] button: & 1 & $\mathrm{x}$ & & & 9/4/96 \\
\hline & & & 30 & Test 'Box' radio button: & 1 & $\mathrm{x}$ & & & $9 / 4 / 96$ \\
\hline & & & 31 & Test [Vault B] button: & 1 & $\mathrm{x}$ & & & $9 / 4 / 96$ \\
\hline & & & 32 & Load Data Script F14.02-32U & 1 & $\mathrm{x}$ & & & 9/4/96 \\
\hline & & & 33 & $\begin{array}{l}\text { DMSCOM CL message received - PIN } \\
\text { 'TST-F14-BX-002' at Box NDE. }\end{array}$ & 1 & $\mathrm{x}$ & & & $9 / 4 / 96$ \\
\hline & & & 34 & Test [Commit] button: & & & OPEN & same as F14.01-34 & \\
\hline & & & 35 & $\begin{array}{l}\text { Test [Vault B] button (current location of PIN } \\
\text { with no previous NDE records): }\end{array}$ & 1. & $\mathrm{x}$ & & & $9 / 4 / 96$ \\
\hline & & & 36 & Test [Waste Record] button for new PIN: & 1 & $\mathrm{x}$ & & & $9 / 4 / 96$ \\
\hline & & & 37 & $\begin{array}{l}\text { Close Container and Content Record (WASTE) } \\
\text { Screen: }\end{array}$ & 1 & $\mathrm{x}$ & & & 9/4/96 \\
\hline & & & 38 & Test [PhysComp] button for new PIN: & 1 & $\mathrm{x}$ & & . & $9 / 4 / 96$ \\
\hline & & & 39 & Close Physical Component Screen: & 1 & $\mathrm{x}$ & & & 9/4/96 \\
\hline & & & 40 & Test [Rad Detail] button for new PIN: & 1 & $\mathrm{x}$ & & & 9/4/96 \\
\hline & & & 41 & $\begin{array}{l}\text { Close Radioactive Waste Container Detail } \\
\text { Record Screen: }\end{array}$ & 1 & $\mathrm{x}$ & & & $9 / 4 / 96$ \\
\hline & & & 42 & Test Location field for updatability: & 1 & $\mathrm{x}$ & & & $9 / 4 / 96$ \\
\hline
\end{tabular}




\begin{tabular}{|c|c|c|c|c|c|c|c|c|c|}
\hline PROC \# & $\begin{array}{l}\text { PROCEDURE } \\
\text { NAME }\end{array}$ & $\begin{array}{c}\text { CASE } \\
\#\end{array}$ & $\begin{array}{c}\text { STE } \\
\text { P } \\
\#\end{array}$ & STEP NAME & $\begin{array}{c}\text { REV } \\
\#\end{array}$ & $\begin{array}{c}\text { PASS } \\
\text { 1st } \\
\text { TEST }\end{array}$ & $\begin{array}{l}\text { PASS } \\
\text { RETEST }\end{array}$ & COMMENTS & $\begin{array}{l}\text { SIGNOFF } \\
\text { DATE }\end{array}$ \\
\hline \multirow[t]{12}{*}{ DMS-F14 } & \multirow[t]{12}{*}{ NDE screen (DMSS0201) } & \multirow[t]{12}{*}{.02} & 43 & Test Outer Dram PIN field for updatability: & 1 & $\mathrm{X}$ & & & $9 / 4 / 96$ \\
\hline & & & 44 & $\begin{array}{l}\text { Test Date field for updatability, format, and } \\
\text { valid value: }\end{array}$ & 1 & $\mathrm{X}$ & & . & $9 / 4 / 96$ \\
\hline & & & 45 & Test Operator field for updatability: & 1 & $\mathrm{X}$ & & & $9 / 4 / 96$ \\
\hline & & & 46 & $\begin{array}{l}\text { Test updatability, case restriction, format, and } \\
\text { length of Video Disk File field: }\end{array}$ & 1 & $\mathrm{X}$ & & & $9 / 4 / 96$ \\
\hline & & & 47 & $\begin{array}{l}\text { Test updatability, case restriction, format, and } \\
\text { length of Video Tape No. freld: }\end{array}$ & 1 & $\mathrm{X}$ & & & $9 / 4 / 96$ \\
\hline & & & 48 & $\begin{array}{l}\text { Test updatability, case restriction, format, and } \\
\text { length of Video Disk No. field: }\end{array}$ & 1 & $x$ & & & $9 / 4 / 96$ \\
\hline & & & 49 & $\begin{array}{l}\text { Test updatability, case restriction, format, and } \\
\text { length of Video Tape Start field: }\end{array}$ & 1 & $\mathrm{x}$ & & & $9 / 4 / 96$ \\
\hline & & & 50 & $\begin{array}{l}\text { Test updatability, case restriction, format, and } \\
\text { length of NDE Comments field: }\end{array}$ & 1 & $\mathrm{X}$ & & & $9 / 4 / 96$ \\
\hline & & & 51 & $\begin{array}{l}\text { Test updatability, case restriction, format, and } \\
\text { length of Contents Verified field: }\end{array}$ & 1 & $\mathrm{X}$ & & . & $9 / 4 / 96$ \\
\hline & & & 52 & $\begin{array}{l}\text { Test updatability, case restriction, format, and } \\
\text { length of Compliant field: }\end{array}$ & 1 & $\mathbf{x}$ & & & $9 / 4 / 96$ \\
\hline & & & 53 & Load Data Soript F14.02-53U. & 1 & $\mathrm{X}$ & & & $9 / 4 / 96$ \\
\hline & & & 54 & $\begin{array}{l}\text { DMSCOM CL messages received - PIN } \\
\text { TST-F14-WP-004 at Vault A, PIN } \\
\text { 'TST-F14-OD-002' at PAN B, PIN } \\
\text { 'TST-F14-PD-005' at Vault B. }\end{array}$ & 1 & $x$ & & & $9 / 4 / 96$ \\
\hline
\end{tabular}




\begin{tabular}{|c|c|c|c|c|c|c|c|c|c|}
\hline PROC \# & $\begin{array}{l}\text { PROCEDURE } \\
\text { NAME }\end{array}$ & $\underset{\#}{\text { CASE }}$ & $\begin{array}{c}\text { STE } \\
\mathbf{P} \\
\#\end{array}$ & STEP NAME & $\begin{array}{c}\text { REV } \\
\#\end{array}$ & $\begin{array}{c}\text { PASS } \\
\text { 1st } \\
\text { TEST }\end{array}$ & $\begin{array}{c}\text { PASS } \\
\text { RETEST }\end{array}$ & COMMENTS & $\begin{array}{l}\text { SIGNOFF } \\
\text { DATE }\end{array}$ \\
\hline \multirow[t]{16}{*}{ DMS-F14 } & \multirow[t]{16}{*}{ NDE screen (DMSS0201) } & \multirow[t]{16}{*}{02} & 55 & Test [insert] button: & & & OPEN & same as F14.01-55 & \\
\hline & & & 56 & Test for required data fields: & 1 & $\mathrm{x}$ & & & $9 / 4 / 96$ \\
\hline & & & 57 & Test [Commit] button: & 1 & $\mathrm{x}$ & & & $9 / 4 / 96$ \\
\hline & & & 58 & Test [Vault B] button: & 1 & $\mathrm{x}$ & & & $9 / 4 / 96$ \\
\hline & & & 60 & Test Delete function for newly entered record: & 1 & $\mathrm{x}$ & & & $9 / 4 / 96$ \\
\hline & & & 61 & Test Rollback function: & 1 & $\mathrm{x}$ & & & $9 / 4 / 96$ \\
\hline & & & 62 & Test [Box] button: & 1 & $\mathrm{x}$ & & & $9 / 4 / 96$ \\
\hline & & & 63 & Test 'Box' radio button: & 1 & $\mathrm{x}$ & & & $9 / 4 / 96$ \\
\hline & & & 64 & Create new record for box PIN: & 1 & $\mathrm{x}$ & & & $9 / 4 / 96$ \\
\hline & & & 65 & Test 'Drum' radio button: & 1 & $\mathbf{x}$ & & & $9 / 4 / 96$ \\
\hline & & & 66 & Test scroll bar functionality: & 1 & $\mathrm{x}$ & & & $9 / 4 / 96$ \\
\hline & & & 67 & Test [Select Item] button: & 1 & $\mathrm{x}$ & & & $9 / 4 / 96$ \\
\hline & & & 68 & Test screen for scrolling through records: & 1 & $\mathrm{x}$ & & & $9 / 4 / 96$ \\
\hline & & & 69 & Test Delete function: & 1 & $\mathrm{x}$ & & & $9 / 4 / 96$ \\
\hline & & & 70 & $\begin{array}{l}\text { Test Commit function with unentered new } \\
\text { record: }\end{array}$ & 1 & $\mathrm{x}$ & & & $9 / 4 / 96$ \\
\hline & & & 71 & Load Data Script F.14:02-71U. & 1 & $\mathrm{x}$ & & & $9 / 4 / 96$ \\
\hline
\end{tabular}




\begin{tabular}{|c|c|c|c|c|c|c|c|c|c|}
\hline PROC \# & $\begin{array}{l}\text { PROCEDURE } \\
\text { NAME }\end{array}$ & $\underset{\#}{\text { CASE }}$ & $\begin{array}{l}\text { STE } \\
\mathbf{P} \\
\#\end{array}$ & STEP NAME & $\underset{\#}{\mathrm{REV}}$ & $\begin{array}{l}\text { PASS } \\
\text { 1st } \\
\text { TEST }\end{array}$ & $\begin{array}{c}\text { PASS } \\
\text { RETEST }\end{array}$ & COMMENTS & $\begin{array}{l}\text { SIGNOFF } \\
\text { DATE }\end{array}$ \\
\hline \multirow[t]{12}{*}{ DMS-F14 } & \multirow[t]{12}{*}{ NDE screen (DMSS0201) } & \multirow[t]{12}{*}{02} & 72 & $\begin{array}{l}\text { DMSCOM CL messages received - PIN } \\
\text { 'TST-F14-PD-001' at Vault B, PIN } \\
\text { 'TST-F14-BX-002' at Box NDA. }\end{array}$ & 1 & $\mathrm{x}$ & & & $9 / 4 / 96$ \\
\hline & & & 73 & Test [Vault A] button: & 1 & $\mathrm{x}$ & & & $9 / 4 / 96$ \\
\hline & & & 74 & Create NDE Record: & 1 & $\mathrm{X}$ & & & $9 / 4 / 96$ \\
\hline & & & 75 & $\begin{array}{l}\text { Test Current NDE Results display for selection } \\
\text { of newest container at location: }\end{array}$ & 1 & $\mathrm{x}$ & & & $9 / 4 / 96$ \\
\hline & & & 76 & Load Data Script. F14.02-76U. & 1 & $\mathrm{x}$ & & & $9 / 4 / 96$ \\
\hline & & & 77 & $\begin{array}{l}\text { DMSCOM CL messages received - PIN } \\
\text { 'TST-F14-EC-001' at Vault A, PIN } \\
\text { 'TST-F14-WP-002' at Vault B, PIN } \\
\text { 'TST-F14-WP-007' at Box NDE, } \\
\text { 'TST-F14-BX-002' in transit, 'T }\end{array}$ & 1 & $\mathrm{x}$ & $=$ & & $9 / 4 / 96$ \\
\hline & & & 79 & View previous NDE results for box records: & 1 & $\mathrm{x}$ & & & $9 / 4 / 96$ \\
\hline & & & 80 & Test Previous Box PIN for updatability: & 1 & $\mathrm{x}$ & & & $9 / 4 / 96$ \\
\hline & & & 81 & $\begin{array}{l}\text { Test Previous Box Results Count for } \\
\text { updatability: }\end{array}$ & 1 & $\mathrm{x}$ & & & $9 / 4 / 96$ \\
\hline & & & 82 & Test Previous Box Results to insert a record: & 1 & $\mathrm{x}$ & & & 9/4/96 \\
\hline & & & 83 & View previous NDE results for drum records: & 1 & $\mathrm{x}$ & & & $9 / 4 / 96$ \\
\hline & & & 84 & Test Previous Drum PIN for updatability: & 1 & $\mathrm{x}$ & & & $9 / 4 / 96$ \\
\hline
\end{tabular}




\begin{tabular}{|c|c|c|c|c|c|c|c|c|c|}
\hline PROC \# & $\begin{array}{c}\text { PROCEDURE } \\
\text { NAME }\end{array}$ & $\underset{\#}{\text { CASE }}$ & $\begin{array}{c}\text { STE } \\
\mathbf{P} \\
\# \\
\end{array}$ & STEP NAME & $\underset{\#}{\text { REV }}$ & $\begin{array}{c}\text { PASS } \\
\text { 1st } \\
\text { TEST }\end{array}$ & $\begin{array}{l}\text { PASS } \\
\text { RETEST }\end{array}$ & COMMENTS & $\begin{array}{l}\text { SIGNOFF } \\
\text { DATE }\end{array}$ \\
\hline \multirow[t]{11}{*}{ DMS-F14 } & \multirow[t]{11}{*}{ NDE screen (DMSS0201) } & \multirow[t]{11}{*}{02} & 85 & $\begin{array}{l}\text { Test Previous Drum Resuits Count for } \\
\text { updatability: }\end{array}$ & 1 & $x$ & & & $9 / 4 / 96$ \\
\hline & & & 86 & Test Previous Drum Results to insert a record: & 1 & $\mathrm{x}$ & & & $9 / 4 / 96$ \\
\hline & & & 87 & Query for a Previous NDE Results record: & 1 & $\mathrm{X}$ & & & $9 / 4 / 96$ \\
\hline & & & 89 & $\begin{array}{l}\text { Test }[<][>][<<][>>] \text { buttons in Previous } \\
\text { NDE Results block: }\end{array}$ & 1 & $x$ & & & $9 / 4 / 96$ \\
\hline & & & 90 & Test [Exit] button (no uncommitted changes): & 1 & $\mathrm{x}$ & & & $9 / 4 / 96$ \\
\hline & & & 91 & Test [Vault A] button: & 1 & $x$ & & & $9 / 4 / 96$ \\
\hline & & & 92 & $\begin{array}{l}\text { Edit PIN number displayed and confirm } \\
\text { validity (waste drum PIN with no previous } \\
\text { NDE records, not at NDE vault location): }\end{array}$ & 1 & & & accept as is & $9 / 4 / 96$ \\
\hline & & & 93 & Redisplay PIN at Vault A location: & 1 & $\mathrm{x}$ & & & 9/4/96 \\
\hline & & & 94 & Test [Commit] button: & 1 & $\mathrm{x}$ & & & $9 / 4 / 96$ \\
\hline & & & 95 & $\begin{array}{l}\text { Edit PIN number displayed and confirm } \\
\text { validity) (Box PIN with previous NDE } \\
\text { records): }\end{array}$ & 1 & $x$ & . & & $9 / 4 / 96$ \\
\hline & & & 96 & $\begin{array}{l}\text { Edit PIN number displayed and confirm } \\
\text { validity (Waste drum PIN with previous NDE } \\
\text { records): }\end{array}$ & 1 & $x$ & & & $9 / 4 / 96$ \\
\hline
\end{tabular}


MS PHASE 1 TEST REPORT

APPENDIX B: FUNCTIONAL TEST RESULTS
DOC NUMBER: HNE-SD-WM-TRP-287

REVISION: 0

DATE: $9 / 27 / 96$

\begin{tabular}{|c|c|c|c|c|c|c|c|c|c|}
\hline PROC \# & $\begin{array}{l}\text { PROCEDURE } \\
\text { NAME }\end{array}$ & $\begin{array}{c}\text { CASE } \\
\#\end{array}$ & $\begin{array}{l}\text { STE } \\
\mathbf{P} \\
\#\end{array}$ & STEP NAME & $\begin{array}{c}\text { REV } \\
\#\end{array}$ & $\begin{array}{c}\text { PASS } \\
\text { 1st } \\
\text { TEST }\end{array}$ & $\begin{array}{l}\text { PASS } \\
\text { RETEST }\end{array}$ & COMMENTS & $\begin{array}{l}\text { SIGNOFF } \\
\text { DATE }\end{array}$ \\
\hline \multirow[t]{14}{*}{ DMS-F14 } & \multirow[t]{14}{*}{ NDE screen (DMSS0201) } & \multirow[t]{14}{*}{02} & 97 & $\begin{array}{l}\text { Edit PIN number displayed and confirm } \\
\text { validity (PIN with no previous NDE records, } \\
\text { use code 'EC', currently at Vault A location): }\end{array}$ & 1 & $X$ & & & $9 / 4 / 96$ \\
\hline & & & 98 & Test [Exit] button: & 1 & $X$ & & & $9 / 4 / 96$ \\
\hline & & & 99 & Test [Vault A] button: & 1 & $X$ & & accept as is & $9 / 4 / 96$ \\
\hline & & & 100 & Retest [Vault A] button: & 1 & $\mathrm{X}$ & & & $9 / 4 / 96$ \\
\hline & & & 101 & $\begin{array}{l}\text { Test [PhysComp] button (Current NDE Item is } \\
\text { empty drum): }\end{array}$ & 1 & $\mathrm{x}$ & & & $9 / \dot{4} / 96$ \\
\hline & & & 102 & $\begin{array}{l}\text { Test [Rad Detail] button (Current NDE Item is } \\
\text { empty drum): }\end{array}$ & 1 & $\mathrm{X}$ & & . & $9 / 4 / 96$ \\
\hline & & & 103 & Load Data Script F14.02-103U & 1 & $x$ & & & $9 / 4 / 96$ \\
\hline & & & 104 & $\begin{array}{l}\text { DMSCOM CL messages received - PIN } \\
\text { 'TST-F14-EC-001' at Empty Container } \\
\text { Storage, PIN 'TST-FI4-WP-002' at AS/RS. }\end{array}$ & 1 & $\mathbf{x}$ & & & $9 / 4 / 96$ \\
\hline & & & 105 & Test [Box] button: & 1 & $\mathbf{x}$ & & & $9 / 4 / 96$ \\
\hline & & & 106 & $\begin{array}{l}\text { Confirm validity of current PIN (drum PIN at } \\
\text { Box location): }\end{array}$ & 1 & $\mathbf{x}$ & & & $9 / 4 / 96$ \\
\hline & & & 107 & Test [Exit] button: & 1 & $\mathrm{X}$ & & & $9 / 4 / 96$ \\
\hline & & & 108 & Exit the DMS system. & 1 & $\mathbf{X}$ & & & $9 / 4 / 96$ \\
\hline & & & 109 & Perform post-test database query 'f1 $4.02 q a^{\prime}$ '. & 1 & $\mathrm{X}$ & & & $9 / 4 / 96$ \\
\hline & & & 110 & $\begin{array}{l}\text { Close test database, DMSCOM, and PCS } \\
\text { simulator Dynacomm instances. }\end{array}$ & 1 & $X$ & & . & $9 / 4 / 96$ \\
\hline
\end{tabular}




\begin{tabular}{|c|c|c|c|c|c|c|c|c|c|}
\hline PROC \# & $\begin{array}{l}\text { PROCEDURE } \\
\text { NAME }\end{array}$ & $\underset{\#}{\text { CASE }}$ & $\begin{array}{c}\text { STE } \\
\mathrm{P} \\
\# \\
\end{array}$ & STEP NAME & $\begin{array}{c}\text { REV } \\
\#\end{array}$ & $\begin{array}{c}\text { PASS } \\
\text { 1st } \\
\text { TEST }\end{array}$ & $\begin{array}{c}\text { PASS } \\
\text { RETEST }\end{array}$ & COMMENTS & $\begin{array}{l}\text { SIGNOFF } \\
\text { DATE }\end{array}$ \\
\hline \multirow[t]{14}{*}{ DMS-F15 } & \multirow[t]{14}{*}{ NDA screen (DMSS0202) } & \multirow[t]{14}{*}{01} & 1 & Load Data Script F15.01-01U & 0 & $\mathrm{X}$ & & & $5 / 30 / 96$ \\
\hline & & & 2 & $\begin{array}{l}\text { Logon to DMS system as a user (USER1) with } \\
\text { test role UPDATE privileges. }\end{array}$ & 1 & $\mathrm{x}$ & & & $5 / 30 / 96$ \\
\hline & & & 3 & $\begin{array}{l}\text { Select the NDA screen (DMSS0202) from the } \\
\text { DMS Main Menu: }\end{array}$ & 1 & OPEN & & 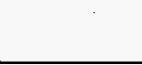 & \\
\hline & & & 4 & Test [NDA Results] button; no PIN displayed: & 0 & $\mathrm{x}$ & & & $5 / 30 / 96$ \\
\hline & & & 5 & Test [Isotopic Data] button; no PIN displayed: & 0 & $\mathrm{x}$ & & & $5 / 30 / 96$ \\
\hline & & & 6 & Test PIN field for required or optional entry: & 0 & $\mathrm{x}$ & & & $5 / 30 / 96$ \\
\hline & & & 7 & Test PIN field for enterability: & 0 & $\mathrm{x}$ & & & $5 / 30 / 96$ \\
\hline & & & 8 & Test PIN freld for insertability: & 0 & $\mathrm{x}$ & & & $5 / 30 / 96$ \\
\hline & & & 9 & Test [List of Values]. button for PIN field: & 0 & $\mathrm{x}$ & & & $5 / 30 / 96$ \\
\hline & & & 11 & $\begin{array}{l}\text { Test up and down arrow navigational keys for } \\
\text { selected PIN: }\end{array}$ & 0 & $\mathrm{x}$ & & & $5 / 30 / 96$ \\
\hline & & & 12 & Test selected PIN for editability: & 0 & $\mathrm{x}$ & & & $5 / 30 / 96$ \\
\hline & & & 13 & $\begin{array}{l}\text { Test tabbing sequence; test Outer Drum PIN } \\
\text { field for Autohint: }\end{array}$ & 0 & $\mathrm{x}$ & & & $5 / 30 / 96$ \\
\hline & & & 14 & $\begin{array}{l}\text { Test tabbing sequence; test Profile ID field for } \\
\text { Autohint: }\end{array}$ & 2 & & $\mathrm{x}$ & & $9 / 4 / 96$ \\
\hline & & & 15 & Test Assay No. field for Autohint: & 0 & $\mathrm{x}$ & & & $5 / 30 / 96$ \\
\hline
\end{tabular}


REVISION: 0

DATE: $9 / 27 / 96$

\begin{tabular}{|c|c|c|c|c|c|c|c|c|c|}
\hline PROC \# & $\begin{array}{l}\text { PROCEDURE } \\
\text { NAME }\end{array}$ & $\underset{\#}{\text { CASE }}$ & $\begin{array}{c}\text { STE } \\
\mathbf{P} \\
\# \\
\end{array}$ & STEP NAME & $\underset{\#}{\text { REV }}$ & $\begin{array}{c}\text { PASS } \\
\text { 1st } \\
\text { TEST }\end{array}$ & $\begin{array}{c}\text { PASS } \\
\text { RETEST }\end{array}$ & COMMENTS & $\begin{array}{l}\text { SIGNOFF } \\
\text { DATE }\end{array}$ \\
\hline \multirow[t]{14}{*}{ DMS-F15 } & \multirow[t]{14}{*}{ NDA screen (DMSS0202) } & \multirow[t]{14}{*}{01} & 16 & Test Assay Date field for Autohint: & 0 & $\mathrm{X}$ & & & $5 / 30 / 96$ \\
\hline & & & 17 & $\begin{array}{l}\text { Test NLLL Nuclide Detected field for } \\
\text { Autohint: }\end{array}$ & 0 & $x$ & & & $5 / 30 / 96$ \\
\hline & & & 18 & Test Profile Used field for Autohint: & 0 & $\mathrm{x}$ & & & $5 / 30 / 96$ \\
\hline & & & 19 & Test Revisit Required field for Autohint: & 0 & $\mathrm{x}$ & & - & $5 / 30 / 96$ \\
\hline & & & 20 & Test Container Type field for Autohint: & 0 & $\mathrm{x}$ & & & $5 / 30 / 96$ \\
\hline & & & 21 & Test Generator Description field for Autohint: & 0 & $x$ & & & $5 / 30 / 96$ \\
\hline & & & 22 & Test Generator Comments field for Autohint: & 0 & $\mathrm{x}$ & & & $5 / 30 / 96$ \\
\hline & & & 23 & Test Secondary Waste Type field for Autohint: & 1 & & $\mathrm{X}$ & . & $6 / 20 / 96$ \\
\hline & & & 25 & Test Waste Category field for Autohint: & 0 & $\mathrm{x}$ & & & $5 / 30 / 96$ \\
\hline & & & 26 & $\begin{array}{l}\text { Test Ratio Check Acceptable field for } \\
\text { Autohint: }\end{array}$ & 0 & $\mathrm{x}$ & & & $5 / 30 / 96$ \\
\hline & & & 27 & $\begin{array}{l}\text { Test Isotopic Confidence Check freld for } \\
\text { Autohint: }\end{array}$ & 1 & & & $\begin{array}{l}\text { accept as is - } \\
\text { reevaluate: Phase } 2\end{array}$ & $6 / 20 / 96$ \\
\hline & & & 28 & Test Total PE Ci field for Autohint: & 1 & & & $\begin{array}{l}\text { accept as is - } \\
\text { reevaluate: Phase } 2\end{array}$ & $6 / 20 / 96$ \\
\hline & & & 29 & Test Total FGE field for Autohint: & 1 & & & $\begin{array}{l}\text { accept as is - } \\
\text { reevaluate: Phase } 2\end{array}$ & $6 / 20 / 96$ \\
\hline & & & 30 & Test Thermal Power field for Autohint: & 1 & & & $\begin{array}{l}\text { accept as is - } \\
\text { reevaluate: Phase } 2\end{array}$ & $6 / 20 / 96$ \\
\hline
\end{tabular}




\begin{tabular}{|c|c|c|c|c|c|c|c|c|c|}
\hline PROC \# & $\begin{array}{l}\text { PROCEDURE } \\
\text { NAME }\end{array}$ & $\begin{array}{c}\text { CASE } \\
\#\end{array}$ & $\begin{array}{c}\text { STE } \\
\mathbf{P} \\
\# \\
\end{array}$ & STEP NAME & $\underset{\#}{\text { REV }}$ & $\begin{array}{c}\text { PASS } \\
\text { 1st } \\
\text { TEST }\end{array}$ & $\begin{array}{c}\text { PASS } \\
\text { RETEST }\end{array}$ & COMMENTS & $\begin{array}{c}\text { SIGNOFF } \\
\text { DATE }\end{array}$ \\
\hline \multirow[t]{13}{*}{ DMS-F15 } & \multirow[t]{13}{*}{ NDA screen (DMSSO202) } & \multirow[t]{13}{*}{01} & 31 & Test [NDA Results] button: & 0 & $\mathrm{x}$ & & $\begin{array}{l}\text { accept as is - } \\
\text { reevalute: Phase } 2\end{array}$ & $5 / 30 / 96$ \\
\hline & & & $32 \mathrm{~A}$ & Test sereen for record scrolling: & 0 & $\mathrm{x}$ & & $\therefore$ & $5 / 30 / 96$ \\
\hline & & & $32 \mathrm{~B}$ & Test delete record function: & 0 & $\mathrm{x}$ & - & & $5 / 30 / 96$ \\
\hline & & & $32 \mathrm{C}$ & $\begin{array}{l}\text { Test Assay Date field for Autohint and } \\
\text { updatability: }\end{array}$ & 0 & $x$ & & & $5 / 30 / 96$ \\
\hline & & & $32 \mathrm{D}$ & $\begin{array}{l}\text { Test Assay No. field for Autohint and } \\
\text { updatability: }\end{array}$ & 0 & $\mathrm{x}$ & & & $5 / 30 / 96$ \\
\hline & & & $32 \mathrm{E}$ & $\begin{array}{l}\text { Test Waste Category field for Autohint and } \\
\text { updatability: }\end{array}$ & 0 & $\mathrm{x}$ & & & $5 / 30 / 96$ \\
\hline & & & $32 \mathrm{~F}$ & $\begin{array}{l}\text { Test Sec. Waste Type field for Autohint and } \\
\text { updatability: }\end{array}$ & 0 & $x$ & & & $5 / 30 / 96$ \\
\hline & & & $32 \mathrm{G}$ & Test.Profile field for Autohint and updatability: & 0 & $\mathrm{x}$ & & & $5 / 30 / 96$ \\
\hline & & & $32 \mathrm{H}$ & $\begin{array}{l}\text { Test Profile ID field for Autohint and } \\
\text { updatability: }\end{array}$ & 0 & $\mathrm{x}$ & & & $5 / 30 / 96$ \\
\hline & & & $32 I$ & $\begin{array}{l}\text { Test Total Isotopic Records field for Autohint } \\
\text { and updatability: }\end{array}$ & 0 & $\mathrm{x}$ & & & $5 / 30 / 96$ \\
\hline & & & $32 \mathrm{~J}$ & $\begin{array}{l}\text { Test Ratio Pu239/Pu240 field for Autohint and } \\
\text { updatability: }\end{array}$ & 0 & $\mathrm{x}$ & & & $5 / 30 / 96$ \\
\hline & & & $32 \mathrm{~K}$ & Test Revisit field for Autohint and updatability: & 0 & $\mathrm{x}$ & & & $5 / 30 / 96$ \\
\hline & & & $32 \mathrm{~L}$ & $\begin{array}{l}\text { Test Future Eval. Required field for Autohint } \\
\text { and updatability: }\end{array}$ & 0 & $\mathrm{x}$ & & - & $5 / 30 / 96$ \\
\hline
\end{tabular}




\begin{tabular}{|c|c|c|c|c|c|c|c|c|c|}
\hline PROC \# & $\begin{array}{l}\text { PROCEDURE } \\
\text { NAME }\end{array}$ & $\underset{\#}{\text { CASE }}$ & $\begin{array}{c}\text { STE } \\
\mathbf{P} \\
\# \\
\end{array}$ & STEP NAME & $\underset{\#}{\text { REV }}$ & $\begin{array}{c}\text { PASS } \\
\text { 1st } \\
\text { TEST }\end{array}$ & $\begin{array}{l}\text { PASS } \\
\text { RETEST }\end{array}$ & COMMENTS & $\begin{array}{l}\text { SIGNOFF } \\
\text { DATE }\end{array}$ \\
\hline \multirow[t]{12}{*}{ DMS-F15 } & \multirow[t]{12}{*}{ NDA screen (DMSS0202) } & \multirow[t]{12}{*}{01} & $32 \mathrm{M}$ & $\begin{array}{l}\text { Test Thermai Power field for Autohint and } \\
\text { updatability: }\end{array}$ & 0 & $\mathrm{x}$ & & & $5 / 30 / 96$ \\
\hline & & & $32 \mathrm{~N}$ & $\begin{array}{l}\text { Test Total Alpha Ci field for Autohint and } \\
\text { updatability: }\end{array}$ & 0 & $\mathrm{x}$ & & & $5 / 30 / 96$ \\
\hline & & & 320 & $\begin{array}{l}\text { Test Total PE CI field for Autohint and } \\
\text { updatability: }\end{array}$ & 0 & $\mathrm{x}$ & & t & $5 / 30 / 96$ \\
\hline & & & $32 P$ & $\begin{array}{l}\text { Test Total PU FGE field for Autohint and } \\
\text { updatability: }\end{array}$ & 0 & $\mathrm{x}$ & & & $5 / 30 / 96$ \\
\hline & & & $32 \mathrm{Q}$ & $\begin{array}{l}\text { Test TMU Thermal Power field for Autohint } \\
\text { and updatability: }\end{array}$ & 0 & $\mathrm{x}$ & & & $5 / 30 / 96$ \\
\hline & & & $32 \mathrm{R}$ & $\begin{array}{l}\text { Test TMU Alpha } \mathrm{Ci} \text { field for Autohint and } \\
\text { updatability: }\end{array}$ & 0 & $x$ & & & $5 / 30 / 96$ \\
\hline & & & $32 \mathrm{~S}$ & $\begin{array}{l}\text { Test TMU PE Ci freld for Autohint and } \\
\text { updatability: }\end{array}$ & 0 & $\mathrm{x}$ & & & $5 / 30 / 96$ \\
\hline & & & $32 \mathrm{~T}$ & $\begin{array}{l}\text { Test TMU PU FGE field for Autohint and } \\
\text { updatability: }\end{array}$ & 0 & $\mathrm{x}$ & & & $5 / 30 / 96$ \\
\hline & & & $32 \mathrm{U}$ & Test PIN field for Autohint and updatability: & 0 & $\mathrm{x}$ & & & $5 / 30 / 96$ \\
\hline & & & 33 & Test [Return to Main 202 Screen] button: & 0 & $\mathrm{x}$ & & & $5 / 30 / 96$ \\
\hline & & & 34 & Test [lsotopic Data] button for selected PIN: & 0 & $\mathrm{x}$ & & $\begin{array}{l}\text { accept as is - } \\
\text { reevalute during } \\
\text { phase } 2\end{array}$ & $5 / 30 / 96$ \\
\hline & & & $34 \mathrm{~A}$ & Test screen for record scrolling: & 0 & $\mathrm{x}$ & & & $5 / 30 / 96$ \\
\hline
\end{tabular}




\begin{tabular}{|c|c|c|c|c|c|c|c|c|c|}
\hline .PROC \# & $\begin{array}{l}\text { PROCEDURE } \\
\text { NAME }\end{array}$ & $\underset{\#}{\text { CASE }}$ & $\begin{array}{c}\text { STE } \\
\mathbf{P} \\
\# \\
\end{array}$ & STEP NAME & $\underset{\#}{\text { REV }}$ & $\begin{array}{c}\text { PASS } \\
\text { 1st } \\
\text { TEST }\end{array}$ & $\begin{array}{l}\text { PASS } \\
\text { RETEST }\end{array}$ & COMMENTS & $\begin{array}{l}\text { SIGNOFF } \\
\text { DATE }\end{array}$ \\
\hline \multirow[t]{14}{*}{ DMS-F15 } & \multirow[t]{14}{*}{ NDA screen (DMSS0202) } & \multirow[t]{14}{*}{01} & $34 \mathrm{~B}$ & Test delete record function for PIN: & 1 & OPEN & & & \\
\hline & & & $34 \mathrm{C}$ & Test Name field for Autohint and updatability: & 0 & $\mathrm{x}$ & & & $5 / 30 / 96$ \\
\hline & & & $34 \mathrm{D}$ & $\begin{array}{l}\text { Test Quantity field for Autohint and } \\
\text { updatability: }\end{array}$ & 0 & $\mathrm{x}$ & & & $5 / 30 / 96$ \\
\hline & & & $34 \mathrm{E}$ & Test TMU field for Autohint and updatability: & 0 & $\mathrm{x}$ & & & $5 / 30 / 96$ \\
\hline & & & $34 \mathrm{~F}$ & $\begin{array}{l}\text { Test Conf. Check field for Autohint and } \\
\text { updatability: }\end{array}$ & 0 & $\mathrm{x}$ & & & $5 / 30 / 96$ \\
\hline & & & $34 \mathrm{G}$ & $\begin{array}{l}\text { Test Meas. Status field for Autohint and } \\
\text { updatability: }\end{array}$ & 0 & $\mathrm{x}$ & . & & $5 / 30 / 96$. \\
\hline & & & $34 \mathrm{H}$ & $\begin{array}{l}\text { Test PAN Active field for Autohint and } \\
\text { updatability: }\end{array}$ & 0 & $\mathrm{x}$ & & & $5 / 30 / 96$ \\
\hline & & & $34 \mathrm{I}$ & $\begin{array}{l}\text { Test PAN Passive field for Autohint and } \\
\text { updatability: }\end{array}$ & 0 & $\mathrm{x}$ & & & $5 / 30 / 96$ \\
\hline & & & $34 \mathrm{~J}$ & $\begin{array}{l}\text { Test GEA Data field for Autohint and } \\
\text { updatability: }\end{array}$ & 0 & $\mathrm{x}$ & & & $5 / 30 / 96$ \\
\hline & & & $34 \mathrm{~K}$ & Test tabbing sequence: & 0 & $\mathrm{x}$ & & & $5 / 30 / 96$ \\
\hline & & & $34 \mathrm{~L}$ & Test previous block function key: & 0 & $\mathrm{x}$ & & & $5 / 30 / 96$ \\
\hline & & & $34 \mathrm{M}$ & $\begin{array}{l}\text { Test record scrolling function for Isotope } \\
\text { records: }\end{array}$ & 0 & $\mathrm{x}$ & & & $5 / 30 / 96$ \\
\hline & & & $34 \mathrm{~N}$ & Test for insert of isotope record: & 2 & $\mathrm{x}$ & & & 9/4/96 \\
\hline & & & 35 & Test [Return to Main 202 Screen] button: & 0 & $\mathrm{X}$ & & & $5 / 30 / 96$ \\
\hline
\end{tabular}


REVISION: 0

DATE: $9 / 27 / 96$

\begin{tabular}{|c|c|c|c|c|c|c|c|c|c|}
\hline PROC \# & $\begin{array}{l}\text { PROCEDURE } \\
\text { NAME }\end{array}$ & $\underset{\sharp}{\text { CASE }}$ & $\begin{array}{c}\text { STE } \\
\mathbf{P} \\
\# \\
\end{array}$ & STEP NAME & $\begin{array}{c}\text { REV } \\
\#\end{array}$ & $\begin{array}{c}\text { PASS } \\
\text { 1st } \\
\text { TEST }\end{array}$ & $\begin{array}{c}\text { PASS } \\
\text { RETEST }\end{array}$ & COMMENTS & $\begin{array}{c}\text { SIGNOFF } \\
\text { DATE }\end{array}$ \\
\hline \multirow[t]{13}{*}{ DMS-F15 } & \multirow[t]{13}{*}{ NDA screen (DMSS0202) } & \multirow[t]{13}{*}{01} & 36 & Test 'Box' tadio button: & 0 & $\mathrm{x}$ & & & $5 / 30 / 96$ \\
\hline & & & 37 & Test [List of Values] button for box PIN: & 0 & $\mathrm{x}$ & & & $5 / 30 / 96$ \\
\hline & & & 38 & Test 'Drum' radio button: & 0 & $\mathrm{x}$ & $\therefore$ & & $5 / 30 / 95$ \\
\hline & & & 39 & Select Drum PIN: & 0 & $\mathrm{x}$ & & & $5 / 30 / 96$ \\
\hline & & & 41 & Select Profile ID from list: & 1 & & $\mathrm{x}$ & & $6 / 20 / 96$ \\
\hline & & & 42 & Test Exit button with uncommitted changes: & 0 & $\mathrm{x}$ & & & $5 / 30 / 96$ \\
\hline & & & 43 & Test [Request Revisit] button: & 1 & & & $\begin{array}{l}\text { accept.as-is for } \\
\text { Phase } 1\end{array}$ & $6 / 20 / 96$ \\
\hline & & & 44 & Test Commit function: & 0 & $\mathrm{x}$ & & & $5 / 30 / 96$ \\
\hline & & & 46 & Re-Enter Signature Password: & 1 & & $\mathrm{x}$ & & $6 / 20 / 96$ \\
\hline & & & 47 & $\begin{array}{l}\text { Start DMSCOM in its own Dyncaomm screen } \\
\text { by typing 'tstdmscom' at the Dynacomm } \\
\text { prompt. }\end{array}$ & 0 & $\mathrm{x}$ & & & $5 / 30 / 96$ \\
\hline & & & 48 & Retest [Request Revisit] button: & 1 & & $x$ & & $6 / 20 / 96$ \\
\hline & & & 49 & Enter query to retrieve PIN records: & 0 & $\mathrm{x}$ & & & $5 / 30 / 96$ \\
\hline & & & 50 & Re-enter query: & 1 & & $\mathrm{x}$ & & $6 / 20 / 96$ \\
\hline
\end{tabular}




\begin{tabular}{|c|c|c|c|c|c|c|c|c|c|}
\hline PROC \# & $\begin{array}{l}\text { PROCEDURE } \\
\text { NAME }\end{array}$ & $\underset{\#}{\text { CASE }}$ & $\begin{array}{c}\text { STE } \\
\mathbf{P} \\
\# \\
\end{array}$ & STEP NAME & $\underset{\#}{\operatorname{REV}}$ & $\begin{array}{c}\text { PASS } \\
\text { Ist } \\
\text { TEST }\end{array}$ & $\begin{array}{c}\text { PASS } \\
\text { RETEST }\end{array}$ & COMMENTS & $\begin{array}{l}\text { SIGNOFF } \\
\text { DATE }\end{array}$ \\
\hline \multirow[t]{14}{*}{ DMS-F15 } & \multirow[t]{14}{*}{ NDA screen (DMSSO202) } & \multirow[t]{14}{*}{01} & 51 & Cancel query: & 1 & OPEN & & & \\
\hline & & & 52 & $\begin{array}{l}\text { Test Profile ID field for format, length, and } \\
\text { case restriction: }\end{array}$ & 1 & & $\mathrm{x}$ & & $6 / 20 / 96$ \\
\hline & & & 53 & $\begin{array}{l}\text { Enter Signature Password; test Commit } \\
\text { function: }\end{array}$ & 0 & $\mathrm{x}$ & & & $5 / 30 / 96$ \\
\hline & & & 54 & Select another PIN: & 0 & $\mathrm{X}$ & & & $5 / 30 / 96$ \\
\hline & & & 55 & $\begin{array}{l}\text { Enter Signature Password; test Commit } \\
\text { function: }\end{array}$ & 1 & & $\mathrm{x}$ & & $6 / 20 / 96$ \\
\hline & & & 56 & Select another PIN: & 0 & & OPEN & & \\
\hline & & & 57 & $\begin{array}{l}\text { Enter Signature Password; test Commit } \\
\text { function: }\end{array}$ & 0 & $\mathrm{x}$ & & & $5 / 30 / 96$ \\
\hline & & & 58 & $\begin{array}{l}\text { Test Isotopic Confidence Check field for } \\
\text { Autohint: }\end{array}$ & 0 & $\mathrm{x}$ & & & $5 / 30 / 96$ \\
\hline & & & 59 & Test Total PE Ci field for Autohint: & 0 & $\mathrm{x}$ & & & $5 / 30 / 96$ \\
\hline & & & 60 & Test Total FGE field for Autohint: & 0 & $x$ & & & $5 / 30 / 96$ \\
\hline & & & 61 & Test Thermal Power field for Autohint: & 0 & $\mathrm{x}$ & & & $5 / 30 / 96$ \\
\hline & & & 62 & Select another PIN: & 0 & $x$ & & & $5 / 30 / 96$ \\
\hline & & & 63 & $\begin{array}{l}\text { Enter Signature Password; test Commit } \\
\text { function: }\end{array}$ & 0 & $x$ & & & $5 / 30 / 96$ \\
\hline & & & 64 & Select another PIN: & 0 & $x$ & & & $5 / 30 / 96$ \\
\hline
\end{tabular}




\begin{tabular}{|c|c|c|c|c|c|c|c|c|c|}
\hline PROC \# & $\begin{array}{l}\text { PROCEDURE } \\
\text { NAME }\end{array}$ & $\underset{\sharp}{\text { CASE }}$ & $\begin{array}{c}\text { STE } \\
\mathbf{P} \\
\#\end{array}$ & STEP NAME & $\underset{\#}{\mathrm{REV}}$ & $\begin{array}{c}\text { PASS } \\
\text { 1st } \\
\text { TEST }\end{array}$ & $\begin{array}{l}\text { PASS } \\
\text { RETEST }\end{array}$ & COMMENTS & $\begin{array}{l}\text { SIGNOFF } \\
\text { DATE }\end{array}$ \\
\hline \multirow[t]{16}{*}{ DMS-F15 } & \multirow[t]{16}{*}{ NDA screen (DMSS0202) } & \multirow[t]{8}{*}{01} & 65 & $\begin{array}{l}\text { Enter Signature Password; test Commit } \\
\text { function: }\end{array}$ & 0 & $\mathrm{x}$ & & & $5 / 30 / 96$ \\
\hline & & & 66 & Select another PIN: & 0 & $x$ & & & $5 / 30 / 96$ \\
\hline & & & 67 & Select another PIN: & 0 & $\mathrm{x}$ & & & $5 / 30 / 96$ \\
\hline & & & 68 & Select another PIN: & 0 & $x$ & & & $5 / 30 / 96$ \\
\hline & & & 69 & Select another PIN: & 0 & $\mathrm{x}$ & & & $5 / 30 / 96$ \\
\hline & & & 70 & Test [Exit] button: & 0 & $x$ & & & $5 / 30 / 96$ \\
\hline & & & 71 & Exit the DMS system. & 0 & $\mathrm{x}$ & & . & $5 / 30 / 96$ \\
\hline & & & 72 & Run post-test query 'f15.01q a'. & 0 & $x$ & . & & $5 / 30 / 96$ \\
\hline & & \multirow[t]{8}{*}{02} & 1 & Load Data Script F15.02-01U & 0 & $\mathrm{x}$ & & & $5 / 30 / 96$ \\
\hline & & & 2 & $\begin{array}{l}\text { Logon to DMS system as a user (USERI) with } \\
\text { test role UPDATE privileges. }\end{array}$ & 0 & $x$ & & & $5 / 30 / 96$ \\
\hline & & & 3 & $\begin{array}{l}\text { Select the NDA screen (DMSS0202) from the } \\
\text { DMS Main Menu: }\end{array}$ & 0 & $x$ & & & $5 / 30 / 96$ \\
\hline & & & 4 & Test [NDA Results] button; no PIN displayed: & 0 & $x$ & & & $5 / 30 / 96$ \\
\hline & & & 5 & Test [Isotopic Data] button; no PIN displayed: & 0 & $\mathrm{x}$ & & & $5 / 30 / 96$ \\
\hline & & & 6 & Test PIN field for required or optional entry: & 0 & $\mathrm{x}$ & & & $5 / 30 / 96$ \\
\hline & & & 7 & Test PIN field for enterability: & 0 & $\mathrm{x}$ & & & $5 / 30 / 96$ \\
\hline & & & 8 & $\begin{array}{l}- \text { Press Alt }+r, i \text { (menu selection Record, } \\
\text { Insert). }\end{array}$ & 0 & $\mathrm{x}$ & & & $5 / 30 / 96$ \\
\hline
\end{tabular}




\begin{tabular}{|c|c|c|c|c|c|c|c|c|c|}
\hline PROC \# & $\begin{array}{l}\text { PROCEDURE } \\
\text { NAME }\end{array}$ & $\underset{\#}{\mathrm{CASE}}$ & $\begin{array}{c}\text { STE } \\
\mathrm{P} \\
\# \\
\end{array}$ & STEP NAME & $\underset{\#}{\text { REV }}$ & $\begin{array}{c}\text { PASS } \\
\text { Ist } \\
\text { TEST }\end{array}$ & $\begin{array}{c}\text { PASS } \\
\text { RETEST }\end{array}$ & COMMENTS & $\begin{array}{c}\text { SIGNOFF } \\
\text { DATE }\end{array}$ \\
\hline \multirow[t]{16}{*}{ DMS-F15 } & \multirow[t]{16}{*}{ NDA screen (DMSS0202) } & \multirow[t]{16}{*}{02} & 9 & Test [List of Values] button for PIN field: & 0 & $\mathrm{x}$ & & & $5 / 30 / 96$ \\
\hline & & & 10 & Test $[<]$ and $[>]$ buttons for selected PIN: & 0 & $\mathrm{x}$ & & & $5 / 30 / 96$ \\
\hline & & & 11 & $\begin{array}{l}\text { Test up and down arrow navigational keys for } \\
\text { selected PIN: }\end{array}$ & 0 & $\mathrm{x}$ & . & & $5 / 30 / 96$ \\
\hline & & & 12 & Test selected PIN for editability: & 0 & $x$ & & & $5 / 30 / 96$ \\
\hline & & & 13 & $\begin{array}{l}\text { Test tabbing sequence; test Outer Drum PIN } \\
\text { field for Autohint: }\end{array}$ & 0 & $\mathrm{x}$ & & & $5 / 30 / 96$ \\
\hline & & & 14 & $\begin{array}{l}\text { Test tabbing sequence; test Profile ID field for } \\
\text { Autohint: }\end{array}$ & 0 & $\mathrm{x}$ & & & $5 / 30 / 96$ \\
\hline & & & 15 & Test Assay No. freld for Autohint: & 0 & $\mathrm{x}$ & & & $5 / 30 / 96$ \\
\hline & & & 16 & Test Assay Date field for Autohint: & 0 & $x$ & & & $5 / 30 / 96$ \\
\hline & & & 17 & $\begin{array}{l}\text { Test NLLL Nuclide Detected field for } \\
\text { Autohint: }\end{array}$ & 0 & $\mathrm{x}$ & & & $5 / 30 / 96$ \\
\hline & & & 18 & Test Profile Used field for Autohint: & 0 & $\mathrm{x}$ & & & $5 / 30 / 96$ \\
\hline & & & 19 & Test Revisit Required field for Autohint: & 0 & $\mathrm{x}$ & & & $5 / 30 / 96$ \\
\hline & & & 20 & Test Container Type field for Autohint: & 0 & $\mathrm{x}$ & & & $5 / 30 / 96$ \\
\hline & & & 21 & Test Generator Description field for Autohint: & 0 & $\mathrm{x}$ & & . & $5 / 30 / 96$ \\
\hline & & & 22 & Test Generator Comments field for Autohint: & 0 & $\mathrm{x}$ & & & $5 / 30 / 96$ \\
\hline & & & 23 & Test Secondary Waste Type field for Autohint: & 0 & $\mathrm{x}$ & & & $5 / 30 / 96$ \\
\hline & & & 25 & Test Waste Category field for Autohint: & 0 & $\mathrm{x}$ & & & $5 / 30 / 96$ \\
\hline
\end{tabular}




\begin{tabular}{|c|c|c|c|c|c|c|c|c|c|}
\hline PROC \# & $\begin{array}{l}\text { PROCEDURE } \\
\text { NAME }\end{array}$ & $\begin{array}{c}\text { CASE } \\
\#\end{array}$ & $\begin{array}{c}\text { STE } \\
P \\
\# \\
\end{array}$ & STEP NAMR & $\begin{array}{c}\text { REV } \\
\#\end{array}$ & $\begin{array}{c}\text { PASS } \\
\text { Ist } \\
\text { TEST }\end{array}$ & $\begin{array}{l}\text { PASS } \\
\text { RETEST }\end{array}$ & COMMENTS & $\begin{array}{l}\text { SIGNOFF } \\
\text { DATE }\end{array}$ \\
\hline \multirow[t]{14}{*}{ DMS-F15 } & \multirow[t]{14}{*}{ NDA screen (DMSS0202) } & \multirow[t]{14}{*}{02} & 26 & $\begin{array}{l}\text { Test Ratio Check Acceptable field for } \\
\text { Autohint: }\end{array}$ & 0 & $\mathrm{X}$ & & & $5 / 30 / 96$ \\
\hline & & & 27 & $\begin{array}{l}\text { Test Isotopic Confidence Check field for } \\
\text { Autohint: }\end{array}$ & 0 & $\mathrm{x}$ & & & $5 / 30 / 96$ \\
\hline & & & 28 & Test Total PE Ci field for Autohint: & 0 & $\mathrm{X}$ & & & $5 / 30 / 96$ \\
\hline & & & 29 & Test Total FGE field for Autohint: & 0 & $\mathrm{x}$ & & & $5 / 30 / 96$ \\
\hline & & & 30 & Test Thermal Power field for Autohint: & 0 & $\mathrm{X}$ & & & $5 / 30 / 96$ \\
\hline & & & 31 & Test [NDA Results] button: & 0 & $\mathrm{X}$ & . & & $5 / 30 / 96$ \\
\hline & & & $32 \mathrm{~A}$ & Test screen for record scrolling: & 0 & $\mathrm{X}$ & & & $5 / 30 / 96$ \\
\hline & & & $32 \mathrm{~B}$ & Test delete record function: & 0. & $\mathrm{x}$ & & & $5 / 30 / 96$ \\
\hline & & & $32 \mathrm{C}$ & $\begin{array}{l}\text { Test Assay Date field for Autohint and } \\
\text { updatability: }\end{array}$ & 0 & $x$ & & & $5 / 30 / 96$ \\
\hline & & & $32 \mathrm{D}$ & $\begin{array}{l}\text { Test Assay No. field for Autohint and } \\
\text { updatability: }\end{array}$ & 0 & $x$ & & & $5 / 30 / 96$ \\
\hline & & & $32 E$ & $\begin{array}{l}\text { Test Waste Category field for Autohint and } \\
\text { updatability: }\end{array}$ & 0 & $x$ & & & $5 / 30 / 96$ \\
\hline & & & $32 \mathrm{~F}$ & $\begin{array}{l}\text { Test Sec. Waste Type field for Autohint and } \\
\text { updatability: }\end{array}$ & 0 & $x$ & & & $5 / 30 / 96$ \\
\hline & & & $32 G$ & Test Profile field for Autohint and updatability: & 0 & $x$ & & & $5 / 30 / 96$ \\
\hline & & & $32 \mathrm{H}$ & $\begin{array}{l}\text { Test Profile ID field for Autohint and } \\
\text { updatability: }\end{array}$ & 0 & $x$ & & & $5 / 30 / 96$ \\
\hline
\end{tabular}




\begin{tabular}{|c|c|c|c|c|c|c|c|c|c|}
\hline PROC $\#$ & $\begin{array}{l}\text { PROCEDURE } \\
\text { NAME }\end{array}$ & $\begin{array}{c}\text { CASE } \\
\# \\
\end{array}$ & $\begin{array}{c}\text { STE } \\
\mathbf{P} \\
\# \\
\end{array}$ & STEP NAME & $\underset{\#}{\text { REV }}$ & $\begin{array}{c}\text { PASS } \\
\text { 1st } \\
\text { TEST }\end{array}$ & $\begin{array}{c}\text { PASS } \\
\text { RETEST }\end{array}$ & COMMENTS & $\begin{array}{l}\text { SIGNOFE } \\
\text { DATE }\end{array}$ \\
\hline \multirow[t]{12}{*}{ DMS-F15 } & \multirow[t]{12}{*}{ NDA screen (DMSSO202) } & \multirow[t]{12}{*}{02} & $32 I$ & $\begin{array}{l}\text { Test Total Isotopic Records field for Autohint } \\
\text { and updatability: }\end{array}$ & 0 & $\mathrm{x}$ & & & $5 / 30 / 96$ \\
\hline & & & $32 J$ & $\begin{array}{l}\text { Test Ratio Pu239/Pu240 field for Autohint and } \\
\text { updatability: }\end{array}$ & 0 & $\mathrm{x}$ & & & $5 / 30 / 96$ \\
\hline & & & $32 \mathrm{~K}$ & Test Revisit field for Autohint and updatability: & 0 & $\mathrm{x}$ & & & $5 / 30 / 96$ \\
\hline & & & $32 \mathrm{~L}$ & $\begin{array}{l}\text { Test Future Eval. Required field for Autohint } \\
\text { and updatability: }\end{array}$ & 0 & $\mathrm{x}$ & & & $5 / 30 / 96$ \\
\hline & & & $32 \mathrm{M}$ & $\begin{array}{l}\text { Test Thermal Power field for Autohint and } \\
\text { updatability: }\end{array}$ & 0 & $\mathrm{x}$ & & & $5 / 30 / 96$ \\
\hline & & & $32 \mathrm{~N}$ & $\begin{array}{l}\text { Test Total Alpha Ci field for Autohint and } \\
\text { updatability: }\end{array}$ & 0 & $\mathrm{x}$ & & & $5 / 30 / 96$ \\
\hline & & & 320 & $\begin{array}{l}\text { Test Total PE CI field for Autohint and } \\
\text { updatability: }\end{array}$ & 0 & $\mathrm{x}$ & & & $5 / 30 / 96$ \\
\hline & & & $32 \mathrm{P}$ & $\begin{array}{l}\text { Test Total PU FGE field for Autohint and } \\
\text { updatability: }\end{array}$ & 0 & $\mathrm{x}$ & & & $5 / 30 / 96$ \\
\hline & & & $32 Q$ & $\begin{array}{l}\text { Test TMU Thermal Power field for Autohint } \\
\text { and updatability: }\end{array}$ & 0 & $x$ & & & $5 / 30 / 96$ \\
\hline & & & $32 R$ & $\begin{array}{l}\text { Test TMU Alpha Ci field for Autohint and } \\
\text { updatability: }\end{array}$ & 0 & $\mathrm{x}$ & & & $5 / 30 / 96$ \\
\hline & & & $32 S$ & $\begin{array}{l}\text { Test TMU PE Ci field for Autohint and } \\
\text { updatability: }\end{array}$ & 0 & $x$ & & & $5 / 30 / 96$ \\
\hline & & & $32 \mathrm{~T}$ & $\begin{array}{l}\text { Test TMU PU FGE field for Autohint and } \\
\text { updatability: }\end{array}$ & 0 & $x$ & & & $5 / 30 / 96$ \\
\hline
\end{tabular}




\begin{tabular}{|c|c|c|c|c|c|c|c|c|c|}
\hline PROC \# & $\begin{array}{l}\text { PROCEDURE } \\
\text { NAME }\end{array}$ & $\underset{\#}{\text { CASE }}$ & $\begin{array}{c}\text { STE } \\
\mathbf{P} \\
\#\end{array}$ & STEP NAME & $\underset{\#}{\mathrm{REV}}$ & $\begin{array}{c}\text { PASS } \\
\text { 1st } \\
\text { TEST }\end{array}$ & $\begin{array}{c}\text { PASS } \\
\text { RETEST }\end{array}$ & COMMENTS & $\begin{array}{l}\text { SIGNOFF } \\
\text { DATE }\end{array}$ \\
\hline \multirow[t]{15}{*}{ DMS-F15 } & \multirow[t]{15}{*}{ NDA screen (DMSS0202) } & \multirow[t]{15}{*}{02} & $32 \mathrm{U}$ & Test PIN field for Autohint and updatability: & 0 & $\dot{x}$ & & & $5 / 30 / 96$ \\
\hline & & & 33 & Test [Return to Main 202 Screen] button: & 0 & $x$ & & & $5 / 30 / 96$ \\
\hline & & & 34 & Test [Isotopic Data] button for selected PIN: & 0 & $\mathrm{x}$ & & & $5 / 30 / 96$ \\
\hline & & & $34 \mathrm{~A}$ & Test screen for record scrolling: & 0 & $\mathrm{x}$ & & & $5 / 30 / 96$ \\
\hline & & & $34 \mathrm{~B}$ & Test delete record function for PIN: & 0 & $\mathrm{x}$ & & & $5 / 30 / 96$ \\
\hline & & & $34 \mathrm{C}$ & Test Name field for Autohint and updatability: & 0 & $x$ & & & $5 / 30 / 96$ \\
\hline & & & $34 \mathrm{D}$ & $\begin{array}{l}\text { Test Quantity field for Autohint and } \\
\text { updatability: }\end{array}$ & 0 & $\mathrm{x}$ & & & $5 / 30 / 96$ \\
\hline & & & $34 \mathrm{E}$ & Test TMU field for Autohint and updatability: & 0 & $\mathrm{x}$ & & & $5 / 30 / 96$ \\
\hline & & & $34 \mathrm{~F}$ & $\begin{array}{l}\text { Test Conf. Check field for Autohint and } \\
\text { updatability: }\end{array}$ & 0 & $x$ & & & $5 / 30 / 96$ \\
\hline & & & $34 \mathrm{G}$ & $\begin{array}{l}\text { Test Meas. Status field for Autohint and } \\
\text { updatability: }\end{array}$ & 0 & $\mathrm{x}$ & . & & $5 / 30 / 96$ \\
\hline & & & $34 \mathrm{H}$ & $\begin{array}{l}\text { Test PAN Active field for Autohint and } \\
\text { updatability: }\end{array}$ & 0 & $\mathrm{x}$ & & & $5 / 30 / 96$ \\
\hline & & & $34 \mathrm{I}$ & $\begin{array}{l}\text { Test PAN Passive field for Autohint and } \\
\text { updatability: }\end{array}$ & 0 & $\mathrm{x}$ & & & $5 / 30 / 96$ \\
\hline & & & $34 \mathrm{~J}$ & $\begin{array}{l}\text { Test GEA Data field for Autohint and } \\
\text { updatability: }\end{array}$ & 0 & $x$ & & & $5 / 30 / 96$ \\
\hline & & & $34 \mathrm{~K}$ & Test tabbing sequence: & 0 & $x$ & & & $5 / 30 / 96$ \\
\hline & & & $34 \mathrm{~L}$ & Test previous block function key: & 0 & $\mathrm{X}$ & & & $5 / 30 / 96$ \\
\hline
\end{tabular}




\begin{tabular}{|c|c|c|c|c|c|c|c|c|c|}
\hline PROC \# & $\begin{array}{l}\text { PROCEDURE } \\
\text { NAME }\end{array}$ & $\underset{\#}{\text { CASE }}$ & $\begin{array}{l}\text { STE } \\
P \\
\#\end{array}$ & STEP NAME & $\underset{\#}{\mathbf{R E V}}$ & $\begin{array}{l}\text { PASS } \\
\text { 1st } \\
\text { TEST }\end{array}$ & $\begin{array}{c}\text { PASS } \\
\text { RETEST }\end{array}$ & COMMENTS & $\begin{array}{l}\text { SIGNOFF } \\
\text { DATE }\end{array}$ \\
\hline \multirow[t]{15}{*}{ DMS-F15 } & \multirow[t]{15}{*}{ NDA screen (DMSS0202) } & \multirow[t]{15}{*}{02} & $34 \mathrm{M}$ & $\begin{array}{l}\text { Test record serolling function for Isotope } \\
\text { records: }\end{array}$ & 0 & $\mathrm{x}$ & & & $5 / 30 / 96$ \\
\hline & & & $34 \mathrm{~N}$ & Test for insext of isotope record: & 0 & $\mathrm{x}$ & . & & $5 / 30 / 96$ \\
\hline & & & 35 & Test [Return to Main 202 Screen] button: & 0 & $\mathrm{x}$ & & & $5 / 30 / 96$ \\
\hline & & & 36 & Test 'Box' radio button: & 0 & $x$ & & & $5 / 30 / 96$ \\
\hline & & & 37 & Test [List of Values] button for box PIN: & 0 & $x$ & & & $5 / 30 / 96$ \\
\hline & & & 38 & Test 'Drum' radio button: & 0 & $\mathrm{x}$ & & & $5 / 30 / 96$ \\
\hline & & & 39 & Select Drum PIN: & 0 & $\mathrm{x}$ & & & $5 / 30 / 96$ \\
\hline & & & 40 & $\begin{array}{l}\text { Test [List of Values] button for Profile ID } \\
\text { (Profile ID same as Profile Used; Revisit } \\
\text { Required 'Y') }\end{array}$ & 0 & $\mathrm{x}$ & & . & $5 / 30 / 96$ \\
\hline & & & 41 & Select Profile ID from list: & 0 & $\mathrm{x}$ & & & $5 / 30 / 96$ \\
\hline & & & 42 & Test Exit button with uncommitted changes: & 0 & $\mathrm{x}$ & & & $5 / 30 / 96$ \\
\hline & & & 43 & Test [Request Revisit] button: & 0 & $\mathrm{x}$ & & & $5 / 30 / 96$ \\
\hline & & & 44 & Test Commit function: & 0 & $\mathrm{x}$ & & & $5 / 30 / 96$ \\
\hline & & & 45 & $\begin{array}{l}\text { Test Signature Password function (enter. } \\
\text { inaccurate password): }\end{array}$ & 0 & $\mathrm{x}$ & & & $5 / 30 / 96$ \\
\hline & & & 46 & Re-Enter Signature Password: & 0 & $\mathrm{x}$ & & & $5 / 30 / 96$ \\
\hline & & & 47 & $\begin{array}{l}\text { Start DMSCOM in its own Dyncaomm screen } \\
\text { by typing 'tstdmscom' at the Dynacomm } \\
\text { prompt. }\end{array}$ & 0 & $\mathrm{x}$ & & & $5 / 30 / 96$ \\
\hline
\end{tabular}




\begin{tabular}{|c|c|c|c|c|c|c|c|c|c|}
\hline PROC \# & $\begin{array}{l}\text { PROCEDURE } \\
\text { NAME } \\
\end{array}$ & $\underset{\#}{\text { CASE }}$ & $\begin{array}{c}\text { STE } \\
\mathbf{P} \\
\# \\
\end{array}$ & STEP NAME & $\underset{\#}{\text { REV }}$ & $\begin{array}{c}\text { PASS } \\
\text { 1st. } \\
\text { TEST }\end{array}$ & $\begin{array}{l}\text { PASS } \\
\text { RETEST }\end{array}$ & COMMENTS & $\begin{array}{l}\text { SIGNOFF } \\
\text { DATE }\end{array}$ \\
\hline \multirow[t]{15}{*}{ DMS-F15 } & \multirow[t]{15}{*}{ NDA screen (DMSS0202) } & \multirow[t]{15}{*}{02} & 48 & Retest [Request Revisit] button: & 0 & $\mathrm{x}$ & & & $5 / 30 / 96$ \\
\hline & & & 49 & Enter query to retrieve PIN records: & 0 & $\mathrm{x}$ & & & $5 / 30 / 96$ \\
\hline & & & 50 & Re-enter query: & 0 & $\mathrm{x}$ & & & $5 / 30 / 96$ \\
\hline & & & 51 & Cancel query: & 0 & $\mathrm{x}$ & . & . & $5 / 30 / 96$ \\
\hline & & & 52 & $\begin{array}{l}\text { Test Profile ID field for format, length, and } \\
\text { case restriction: }\end{array}$ & 0 & $\mathrm{x}$ & & & $5 / 30 / 96$ \\
\hline & & & 53 & $\begin{array}{l}\text { Enter Signature Password; test Commit } \\
\text { function: }\end{array}$ & 0 & $\mathrm{x}$ & & & $5 / 30 / 96$ \\
\hline & & & 54 & Select another PIN: & 0 & $\mathrm{x}$ & & & $5 / 30 / 96$ \\
\hline & & & 55 & $\begin{array}{l}\text { Enter Signature Password; test Commit } \\
\text { function: }\end{array}$ & 0 & $x$ & & & $5 / 30 / 96$ \\
\hline & & & 56 & Select another PIN: & 0 & $\mathrm{x}$ & & & $5 / 30 / 96$ \\
\hline & & & 57 & $\begin{array}{l}\text { Enter Signature Password; test Commit } \\
\text { function: }\end{array}$ & 0 & $\mathrm{x}$ & & & $5 / 30 / 96$ \\
\hline & & & 58 & $\begin{array}{l}\text { Test lsotopic Confidence Check field for } \\
\text { Autohint: }\end{array}$ & 0 & $\mathrm{x}$ & & & $5 / 30 / 96$ \\
\hline & & & 59 & Test Total PE Ci field for Autohint: & 0 & $\mathrm{x}$ & & & $5 / 30 / 96$ \\
\hline & & & 60 & Test Total FGE field for Autohint: & 0 & $\mathrm{x}$ & & & $5 / 30 / 96$ \\
\hline & & & 61 & Test Thermal Power field for Autohint: & 0 & $\mathrm{x}$ & & & $5 / 30 / 96$ \\
\hline & & & 62 & Select another PIN: & 0 & $\mathrm{x}$ & & & $5 / 30 / 96$ \\
\hline
\end{tabular}


REVISION: 0

DATE: $9 / 27 / 96$

\begin{tabular}{|c|c|c|c|c|c|c|c|c|c|}
\hline PROC \# & $\begin{array}{l}\text { PROCEDURE } \\
\text { NAME }\end{array}$ & $\underset{\#}{\text { CASE }}$ & $\begin{array}{c}\operatorname{STE} \\
\mathbf{P} \\
\# \\
\end{array}$ & STEP NAME & $\underset{\#}{\text { REV }}$ & $\begin{array}{l}\text { PASS } \\
\text { 1st } \\
\text { TEST }\end{array}$ & $\begin{array}{c}\text { PASS } \\
\text { RETEST }\end{array}$ & COMMENTS & $\begin{array}{l}\text { SIGNOFF } \\
\text { DATE }\end{array}$ \\
\hline \multirow[t]{11}{*}{ DMS-F15 } & \multirow[t]{11}{*}{ NDA screen (DMSSO202) } & \multirow[t]{11}{*}{02} & 63 & $\begin{array}{l}\text { Enter Signature Password; test Commit } \\
\text { function: }\end{array}$ & 0 & $\mathrm{x}$ & & & $5 / 30 / 96$ \\
\hline & & & 64 & Select another PIN: & 0 & $\mathrm{x}$ & & & $5 / 30 / 96$ \\
\hline & & & 65 & $\begin{array}{l}\text { Enter Signature Password; test Commit } \\
\text { function: }\end{array}$ & 0 & $\mathrm{x}$ & & & $5 / 30 / 96$ \\
\hline & & & 66 & Select another PIN: & 0 & $\mathrm{x}$ & & & $5 / 30 / 96$ \\
\hline & & & 67 & Select another PIN: & 0 & $x$ & & & $5 / 30 / 96$ \\
\hline & & & 68 & Select another PIN: & 0 & $x$ & & & $5 / 30 / 96$ \\
\hline & & & 69 & Select another PIN: & 0 & $\mathrm{x}$ & & & $5 / 30 / 96$ \\
\hline & & & 70 & Test [Exit] button: & 0 & $\mathrm{x}$ & & & $5 / 30 / 96$ \\
\hline & & & 71 & Exit the DMS system. & 0 & $\mathrm{x}$ & & & $5 / 30 / 96$ \\
\hline & & & 72 & Run post-test query' 'f15.01q a'. & 0 & $\mathrm{x}$ & & & $5 / 30 / 96$ \\
\hline & & & 73 & Shut down SIE simulator. & 0 & $\mathrm{x}$ & & & $5 / 30 / 96$ \\
\hline \multirow[t]{4}{*}{ DMS-F16 } & \multirow[t]{4}{*}{$\begin{array}{l}\text { LLW Drum Status at Entry } \\
\text { (DMSS0311) }\end{array}$} & \multirow[t]{4}{*}{01} & 1 & $\begin{array}{l}\text { Logon to DMS system as a user (USER1) with } \\
\text { test role UPDATE privileges. }\end{array}$ & 0 & $\mathrm{x}$ & & & $3 / 22 / 96$ \\
\hline & & & 2 & $\begin{array}{l}\text { Enter the LLW Drum Status at Entry screen } \\
\text { (DMSS0311) and perform the following tests. }\end{array}$ & 0 & $\mathrm{x}$ & & & $3 / 22 / 96$ \\
\hline & & & 3 & Test the [EXIT] button. & 0 & $x$ & & & $3 / 22 / 96$ \\
\hline & & & 4 & $\begin{array}{l}\text { Run script (f16.01-04u) to change the location } \\
\text { of drum TST-F16-OP-002 from LEW_ENTRY } \\
\text { to LLW_EXIT. }\end{array}$ & 0 & $\mathrm{x}$ & & & $3 / 22 / 96$ \\
\hline
\end{tabular}




\begin{tabular}{|c|c|c|c|c|c|c|c|c|c|}
\hline PROC \# & $\begin{array}{l}\text { PROCEDURE } \\
\text { NAME }\end{array}$ & $\underset{\#}{\text { CASE }}$ & $\begin{array}{c}\text { STE } \\
\mathbf{P} \\
\# \\
\end{array}$ & STEP NAME & $\begin{array}{c}\text { REV } \\
\#\end{array}$ & $\begin{array}{c}\text { PASS } \\
\text { 1st } \\
\text { TEST }\end{array}$ & $\begin{array}{c}\text { PASS } \\
\text { RETEST }\end{array}$ & COMMENTS & $\begin{array}{l}\text { SIGNOFF } \\
\text { DATE }\end{array}$ \\
\hline \multirow[t]{15}{*}{ DMS-F17 } & \multirow[t]{15}{*}{ LLW Sorting Table screen (DMSS0312) } & \multirow[t]{13}{*}{01} & 3 & $\begin{array}{l}\text { Select the LLW Sorting Table screen } \\
\text { (DMSS0312) from the DMS Main Menu: }\end{array}$ & 0 & $\mathrm{x}$ & & & $7 / 09 / 96$ \\
\hline & & & 4 & $\begin{array}{l}\text { Test Parent PIN field for insert on LLW } \\
\text { Sorting Table Screen: }\end{array}$ & 0 & $\mathrm{x}$ & & & $7 / 09 / 96$ \\
\hline & & & 5 & Test [Non-Compliant] button: & 0 & OPEN & & & \\
\hline & & & 6 & $\begin{array}{l}\text { Test LLW Non-Compliant Screen Parent PIN } \\
\text { field for insert: }\end{array}$ & 0 & OPEN & & & · \\
\hline & & & 7 & $\begin{array}{l}\text { Test LLW Non-Compliant Screen Transfer } \\
\text { PIN field for insert: }\end{array}$ & 0 & OPEN & & & \\
\hline & & & 8 & $\begin{array}{l}\text { Test LLW Non-Compliant Screen Packet PIN } \\
\text { field for insert: }\end{array}$ & 0 & $\mathrm{x}$ & & & $7 / 09 / 96$ \\
\hline & & & 9 & Test [Return to Sorting Table Screen] button: & 0 & $x$ & & & $7 / 09 / 96$ \\
\hline & & & 10 & Test [Sample] button: & 0 & $\mathrm{x}$ & & & $7 / 09 / 96^{\circ}$ \\
\hline & & & 11 & Test [Chemcomp] button: & 0 & OPEN & & & \\
\hline & & & 12 & Test [Physcomp] button: & 0 & OPEN & & & \\
\hline & & & 13 & Test [NDE Comment] button: & 0 & OPEN & & & \\
\hline & & & 14 & Test [Exit] button: & 0 & $x$ & 1 & & $7 / 09 / 96$ \\
\hline & & & 15 & Exit the DMS system. & 0 & $\mathrm{x}$ & & & $7 / 09 / 96$ \\
\hline & & \multirow[t]{2}{*}{02} & 1 & RUn WRESTORE. & 0 & $\mathrm{x}$ & & & $7 / 09 / 96$ \\
\hline & & & 2 & $\begin{array}{l}\text { Logon to DMS system as a user (USER1) with } \\
\text { test role UPDATE privileges. }\end{array}$ & 0 & $\mathrm{x}$ & & & $7 / 09 / 96$ \\
\hline
\end{tabular}




\begin{tabular}{|c|c|c|c|c|c|c|c|c|c|}
\hline PROC \# & $\begin{array}{l}\text { PROCEDURE } \\
\text { NAME }\end{array}$ & $\underset{\#}{\text { CASE }}$ & $\begin{array}{c}\text { STE } \\
\mathrm{P} \\
\# \\
\end{array}$ & STEP NAME & $\underset{\#}{\text { REV }}$ & $\begin{array}{l}\text { PASS } \\
\text { 1st } \\
\text { TEST }\end{array}$ & $\begin{array}{l}\text { PASS } \\
\text { RETEST }\end{array}$ & COMMENTS & $\begin{array}{l}\text { SIGNOFF } \\
\text { DATE }\end{array}$ \\
\hline \multirow[t]{14}{*}{ DMS-F17 } & \multirow[t]{14}{*}{ LLW Sorting Table screen (DMSS0312) } & \multirow[t]{14}{*}{02} & 3 & $\begin{array}{l}\text { Select the LLW Sorting Table screen } \\
\text { (DMSS0312) from the DMS Main Menu: }\end{array}$ & 0 & $\mathrm{x}$ & & & $7 / 09 / 96$ \\
\hline & & & 4. & Test [Non-Compliant] button: & 0 & OPEN & & & \\
\hline & & & 5 & Load Data Script F17.02-05U & 0 & $\mathrm{x}$ & & & $7 / 09 / 96$ \\
\hline & & & 6 & Test [Refresh] button: & 0 & $\mathrm{x}$ & & & $7 / 09 / 96$ \\
\hline & & & 7 & Load Data Script F17.02-07U. & 0 & $\mathrm{x}$ & & script correction & $7 / 14 / 96$ \\
\hline & & & 8 & Exit and re-enter LLW Non-Compliant Screen: & 0 & $\mathrm{x}$ & & & $7 / 09 / 96$ \\
\hline & & & 9 & $\begin{array}{l}\text { Test Refresh function at LLW Sorting Table } \\
\text { Screen: }\end{array}$ & 0 & $\mathrm{x}$ & & & $7 / 09 / 96$ \\
\hline & & & 10 & $\begin{array}{l}\text { Exit and re-enter LLW Sorting Table Screen; } \\
\text { verify that correct data set is displayed: }\end{array}$ & 0 & $\mathrm{x}$ & & & $7 / 09 / 96$ \\
\hline & & & $1 I$ & $\begin{array}{l}\text { Select the LLW Non-Compliant Screen from } \\
\text { the LLW Sorting Table Screen; verify that } \\
\text { correct data set is displayed: }\end{array}$ & 0 & $\mathrm{x}$ & & & $7 / 09 / 96$ \\
\hline & & & 12 & Load Data Script F17.02-12U. & 0 & $\mathrm{x}$ & & & $7 / 09 / 96$ \\
\hline & & & 13 & Test [Refresh] button: & 0 & $\mathrm{x}$ & & & $7 / 09 / 96$ \\
\hline & & & 14 & Load Data Script F17.02-14U. & 0 & $\mathrm{x}$ & & & $7 / 09 / 96$ \\
\hline & & & 15 & $\begin{array}{l}\text { Close LLW Non-Compliant Screen; exit and } \\
\text { re-enter LLW Sorting Table Screen; verify that } \\
\text { correct data set is displayed: }\end{array}$ & 0 & $x$ & & & $7 / 09 / 96$ \\
\hline & & & 16 & $\begin{array}{l}\text { Open LLW Non-Compliant Screen; verify that } \\
\text { correct data set is displayed: }\end{array}$ & 0 & $\mathrm{x}$ & & & $7 / 09 / 96$ \\
\hline
\end{tabular}




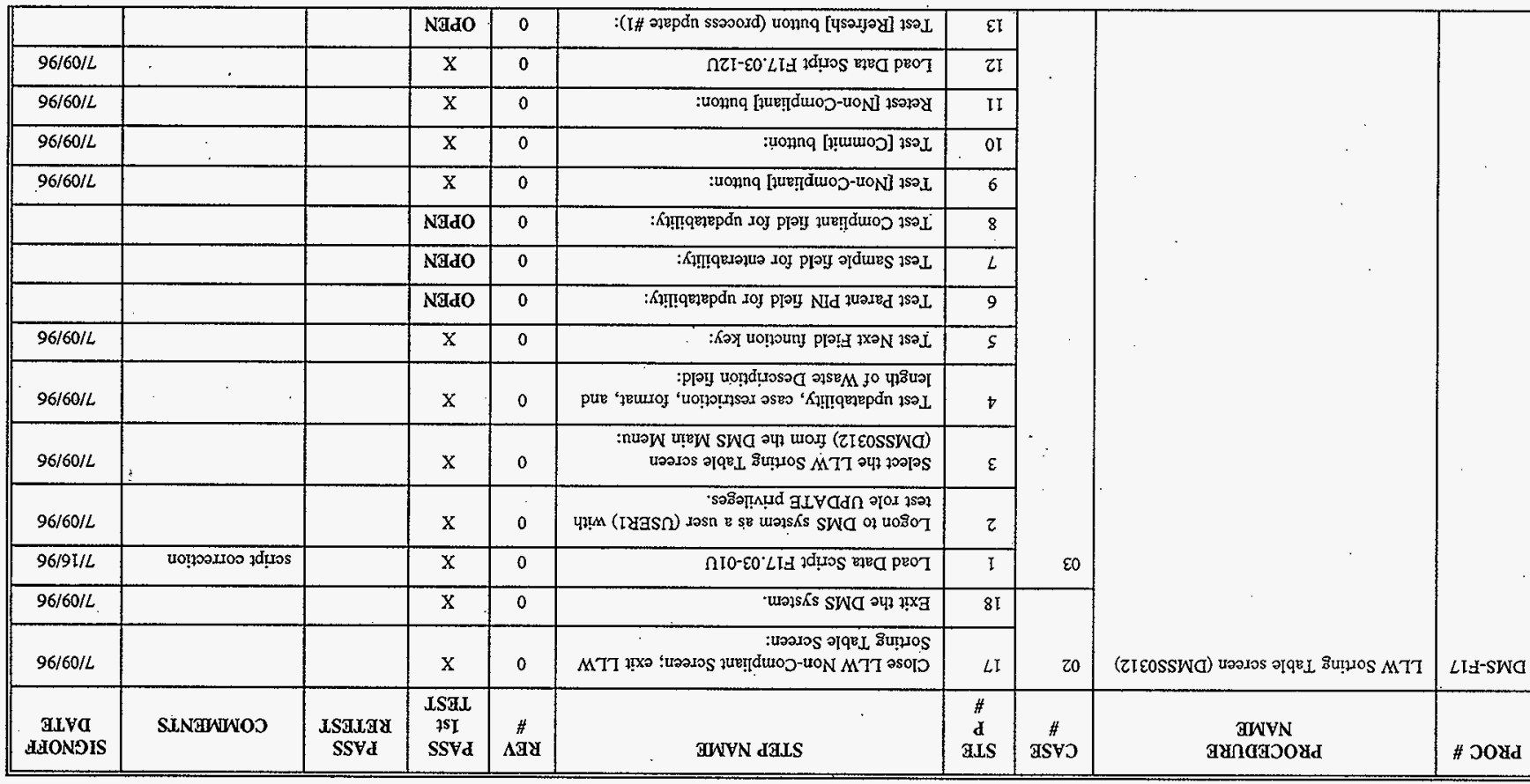

96/LZ/6:GLVO

0 :NOISInay

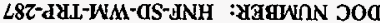

SITASA\& LSGX TYNOLLNAX : LAOdGy LSGL I GSVHd SWC 


\begin{tabular}{|c|c|c|c|c|c|c|c|c|c|}
\hline PROC \# & $\begin{array}{l}\text { PROCEDURE } \\
\text { NAME }\end{array}$ & $\underset{\#}{\text { CASE }}$ & $\begin{array}{c}\text { STE } \\
\mathrm{P} \\
\# \\
\end{array}$ & STEP NAME & $\underset{\#}{\text { REV }}$ & $\begin{array}{l}\text { PASS } \\
\text { 1st } \\
\text { TEST }\end{array}$ & $\begin{array}{c}\text { PASS } \\
\text { RETEST }\end{array}$ & COMMENTS & $\begin{array}{l}\text { SIGNOFF } \\
\text { DATE }\end{array}$ \\
\hline \multirow[t]{14}{*}{ DMS-F17 } & \multirow[t]{14}{*}{ LLW Sorting Table screen (DMSS0312) } & \multirow[t]{14}{*}{03} & 14 & $\begin{array}{l}\text { Test LLW Non-Compliant Screen Parent PIN } \\
\text { field for editability: }\end{array}$ & 0 & $\mathrm{x}$ & & & $7 / 09 / 96$ \\
\hline & & & 15 & $\begin{array}{l}\text { Test Group field for editability, length, case } \\
\text { restriction, and valid data values: }\end{array}$ & 0 & $\mathrm{x}$ & & $\begin{array}{l}\text { accept as-is for } \\
\text { Phase } 1 \text {. }\end{array}$ & $7 / 09 / 96$ \\
\hline & & & 16 & $\begin{array}{l}\text { Test Waste Description field for editability, } \\
\text { length, case restriction, and valid data values: }\end{array}$ & 0 & $x$ & & & $7 / 09 / 96$ \\
\hline & & & 17 & Test Group field for insertability: & 0 & OPEN & & & \\
\hline & & & 18 & Load Data Script F17.03-18U & 0 & OPEN & & & \\
\hline & & & 19 & Test [Refresh] button (process update \#2): & 0 & OPEN & & & \\
\hline & & & 20 & Test Signature Password function: & 0 & OPEN & & & \\
\hline & & & 21 & $\begin{array}{l}\text { Pre-enter Signature Password for next } \\
\text { operation: }\end{array}$ & 0 & $\mathrm{x}$ & & & $7 / 09 / 96$ \\
\hline & & & 22 & Redisplay LLW Non-Compliant Screen: & 0 & $\mathrm{x}$ & & & $7 / 09 / 96$ \\
\hline & & & 23 & $\begin{array}{l}\text { Test Transfer Drum Status for updatability, } \\
\text { field length, and case restriction: }\end{array}$ & 0 & $\mathrm{x}$ & & & $7 / 09 / 96$ \\
\hline & & & $24^{\circ}$ & $\begin{array}{l}\text { Enter Material Group Code and Waste } \\
\text { Description for new packets: }\end{array}$ & 0 & $\mathrm{x}$ & & & $7 / 09 / 96$ \\
\hline & & & 25 - & $\begin{array}{l}\text { Test screen navigation keys in Packet PIN } \\
\text { block: }\end{array}$ & 0 & $\mathrm{x}$ & & & $7 / 09 / 96$ \\
\hline & & & 26 & Test [Retum to Sorting Table Screen] button: & 0 & $\mathrm{x}$ & & & $7 / 09 / 96$ \\
\hline & & & 27 & Retest [Non-Compliant] button: & 0 & $\mathrm{X}$ & & & $7 / 09 / 96$. \\
\hline
\end{tabular}




\begin{tabular}{|c|c|c|c|c|c|c|c|c|c|}
\hline PROC \# & $\begin{array}{l}\text { PROCEDURE } \\
\text { NAME }\end{array}$ & $\underset{\#}{\text { CASE }}$ & $\begin{array}{c}\text { STE } \\
\mathbf{P} \\
\# \\
\end{array}$ & STEP NAME & $\underset{\#}{\text { REV }}$ & $\begin{array}{c}\text { PASS } \\
\text { 1st } \\
\text { TEST }\end{array}$ & $\begin{array}{c}\text { PASS } \\
\text { RETEST }\end{array}$ & COMMENTS & $\begin{array}{l}\text { SIGNOFF } \\
\text { DATE }\end{array}$ \\
\hline \multirow[t]{11}{*}{ DMS-F17 } & \multirow[t]{11}{*}{ LLW Sorting Table screen (DMSS0312) } & \multirow[t]{11}{*}{03} & 28 & Test [Chemcomp] button: & 0 & $\mathrm{x}$ & & & $7 / 09 / 96$ \\
\hline & & & 29 & $\begin{array}{l}\text { Test Chemical Composition Screen ID field for } \\
\text { editability: }\end{array}$ & 0 & $\mathrm{x}$ & & & $7 / 09 / 96$ \\
\hline & & & 30 & $\begin{array}{l}\text { Test Chemical Composition Screen Description } \\
\text { field for editability: }\end{array}$ & 0 & OPEN & & & \\
\hline & & & 31 & $\begin{array}{l}\text { Test Chemical Composition Screen Weight } \\
\text { (kg) field for editablity: }\end{array}$ & 0 & $\mathrm{x}$ & & & $7 / 09 / 96$ \\
\hline & & & 32 & $\begin{array}{l}\text { Test Chemical Composition Screen Weight } \\
\text { Percent field for editablity: }\end{array}$ & 0 & $\mathrm{x}$ & & & $7 / 09 / 96$ \\
\hline & & & 33 & $\begin{array}{l}\text { Test Chemical Composition Screen ID field for } \\
\text { insertability and required field: }\end{array}$ & 0 & $\mathrm{x}$ & & & $7 / 09 / 96$ \\
\hline & & & 34 & $\begin{array}{l}\text { Test [List of Values] button for Chemical } \\
\text { Composition Screen ID field: }\end{array}$ & 0 & $x$ & & & $7 / 09 / 96$ \\
\hline & & & 36 & $\begin{array}{l}\text { Select Chemical Composition ID field value } \\
\text { from Valid values pop-up: }\end{array}$ & 0 & $x$ & & & $7 / 09 / 96$ \\
\hline & & & 37 & $\begin{array}{l}\text { Test Chemical Composition Description field } \\
\text { for enterability, format, length, case } \\
\text { restriction, and valid data value: }\end{array}$ & 0 & OPEN & & . & \\
\hline & & & 38 & $\begin{array}{l}\text { Test Chemical Composition Weight field for } \\
\text { enterability, length, and case restriction: }\end{array}$ & 0 & $\mathrm{x}$ & & & $7 / 09 / 96$ \\
\hline & & & 39 & $\begin{array}{l}\text { Test Chemical Composition Weight field for } \\
\text { valid value format: }\end{array}$ & 0 & OPEN & & & \\
\hline
\end{tabular}




\begin{tabular}{|c|c|c|c|c|c|c|c|c|c|}
\hline PROC \# & $\begin{array}{l}\text { PROCEDURE } \\
\text { NAME }\end{array}$ & $\begin{array}{c}\text { CASE } \\
\#\end{array}$ & $\begin{array}{l}\text { STE } \\
\text { P } \\
\#\end{array}$ & STEP NAME & $\begin{array}{c}\text { REV } \\
\#\end{array}$ & $\begin{array}{l}\text { PASS } \\
\text { 1st } \\
\text { TEST }\end{array}$ & $\begin{array}{l}\text { PASS } \\
\text { RETEST }\end{array}$ & COMMENTS & $\begin{array}{l}\text { SIGNOFF } \\
\text { DATE }\end{array}$ \\
\hline \multirow[t]{12}{*}{ DMS-F17 } & \multirow[t]{12}{*}{ LLW Sorting Table screen (DMSS0312) } & \multirow[t]{12}{*}{03} & 40 & $\begin{array}{l}\text { Test Chemical Composition Weight Percent } \\
\text { field for format, length, and case restriction: }\end{array}$ & 0 & OPEN & & · & \\
\hline & & & 41 & Test duplicate item function in ID block: & 0 & $\mathrm{X}$ & & & $7 / 09 / 96$ \\
\hline & & & 42 & Test Weight (kg) field for required entry: & 0 & OPEN & & & \\
\hline & & & 43 & $\begin{array}{l}\text { Test [Delete] button in Chemical Composition } \\
\text { field: }\end{array}$ & 0 & $\mathrm{X}$ & & . & $7 / 09 / 96$ \\
\hline & & & 44 & Test [Return to Sorting Table Screen] button: & 0 & $\mathrm{X}$ & & & $7 / 09 / 96$ \\
\hline & & & 45 & Test [Physcomp] button: & 0 & $\mathrm{X}$ & & script correction & $7 / 16 / 96$ \\
\hline & & & 46 & $\begin{array}{l}\text { Test Physical Composition Screen Description } \\
\text { field for editability: }\end{array}$ & 0 & $\mathrm{X}$ & & & $7 / 09 / 96$ \\
\hline & & & 47 & $\begin{array}{l}\text { Test Physical Composition Screen Volume } \\
\text { Percent field for editablity: }\end{array}$ & 0 & $\mathrm{X}$ & & & $7 / 09 / 96$ \\
\hline & & & 48 & $\begin{array}{l}\text { Test Physical Composition Screen Weight }(\mathrm{Kg}) \\
\text { field for editablity: }\end{array}$ & 0 & $\mathrm{X}$ & - & & $7 / 09 / 96$ \\
\hline & & & 49 & $\begin{array}{l}\text { Test Physical Composition Screen Description } \\
\text { field for insertability, format, length, and case } \\
\text { restriction: }\end{array}$ & 0 & $\mathrm{X}$ & & & $7 / 09 / 96$ \\
\hline & & & 50 & $\begin{array}{l}\text { Test [List of Values] button for Physical } \\
\text { Composition Screen Description field: }\end{array}$ & 0 & $\mathrm{X}$ & & & $7 / 09 / 96$ \\
\hline & & & 51 & $\begin{array}{l}\text { Test Physical Composition Volume Percent } \\
\text { field for editability, length, and case } \\
\text { restriction: }\end{array}$ & 0 & OPEN & & & \\
\hline
\end{tabular}




\begin{tabular}{|c|c|c|c|c|c|c|c|c|c|}
\hline PROC \# & $\begin{array}{l}\text { PROCEDURE } \\
\text { NAME }\end{array}$ & $\underset{\#}{\text { CASE }}$ & $\begin{array}{c}\text { STE } \\
\mathbf{P} \\
\#\end{array}$ & STEP NAME & $\underset{\#}{\mathbf{R E V}}$ & $\begin{array}{c}\text { PASS } \\
\text { 1st } \\
\text { TEST }\end{array}$ & $\begin{array}{c}\text { PASS } \\
\text { RETEST }\end{array}$ & COMMENTS & $\begin{array}{l}\text { SIGNOFF } \\
\text { DATE }\end{array}$ \\
\hline \multirow[t]{13}{*}{ DMS-F17 } & \multirow[t]{13}{*}{ LLW Sorting Table screen (DMSS0312) } & \multirow[t]{13}{*}{03} & 52 & $\begin{array}{l}\text { Test Physical Composition Weight }(\mathrm{kg}) \text { field } \\
\text { for editability, length, and case restriction: }\end{array}$ & 0 & $\mathrm{x}$ & - & & $7 / 09 / 96$ \\
\hline & & & 53 & $\begin{array}{l}\text { Test Physical Composition Weight }(\mathrm{kg}) \text { field } \\
\text { for format valid value: }\end{array}$ & 0 . & OPEN & & & \\
\hline & & & 54 & $\begin{array}{l}\text { Test [Delete] button in Physical Composition } \\
\text { Screen: }\end{array}$ & 0 & $\mathrm{x}$ & & & $7 / 09 / 96$ \\
\hline & & & 55 & Test [Return to Sorting Table Screen] button: & 0 & $\mathrm{x}$ & & & $7 / 09 / 96$ \\
\hline & & & 56 & Test [NDE Comment] button: & 0 & OPEN & & & \\
\hline & & & 57 & $\begin{array}{l}\text { Test NDE Comment Screen Date field for } \\
\text { updatability: }\end{array}$ & 0 & $\mathrm{x}$ & & & $7 / 09 / 96$ \\
\hline & & & 58 & $\begin{array}{l}\text { Test NDE Comment Screen Comments field } \\
\text { for updatability: }\end{array}$ & 0 & $\mathrm{x}$ & . & & $7 / 09 / 96$ \\
\hline & & & 59 & $\begin{array}{l}\text { Test scroll up and scroll down functions in } \\
\text { NDE Comment Screen: }\end{array}$ & 0 & $\mathrm{x}$ & & & $7 / 09 / 96$ \\
\hline & & & 60 & $\begin{array}{l}\text { Test NDE Comment Screen for insert record } \\
\text { function: }\end{array}$ & 0 & $\mathrm{x}$ & & & $7 / 09 / 96$ \\
\hline & & & 61 & Test [Return to Sorting Table Screen] button: & 0 & $\mathrm{x}$ & & & $7 / 09 / 96$ \\
\hline & & & 62 & $\begin{array}{l}\text { Test for prompt for uncommitted changes on } \\
\text { Exit: }\end{array}$ & 0 & $\mathrm{x}$ & & & $7 / 09 / 96$ \\
\hline & & & 63 & $\begin{array}{l}\text { Enter Signature Password and test [Commit] } \\
\text { button: }\end{array}$ & 0 & OPEN & & & \\
\hline & & & 64 & Retest commit function: & 0 & OPEN & & & \\
\hline
\end{tabular}




\begin{tabular}{|c|c|c|c|c|c|c|c|c|c|}
\hline $\mathrm{PROC} \#$ & $\begin{array}{l}\text { PROCEDURE } \\
\text { NAME }\end{array}$ & $\underset{\#}{\text { CASE }}$ & $\begin{array}{c}\text { STE } \\
\mathbf{P} \\
\#\end{array}$ & STEP NAME & $\underset{\#}{\mathrm{REV}}$ & $\begin{array}{l}\text { PASS } \\
\text { 1st } \\
\text { TEST }\end{array}$ & $\begin{array}{c}\text { PASS } \\
\text { RETEST }\end{array}$ & COMMENTS & $\begin{array}{l}\text { SIGNOFF } \\
\text { DATE }\end{array}$ \\
\hline \multirow{15}{*}{ DMS-F17 } & \multirow[t]{15}{*}{ LLW Sorting Table screen (DMSS0312) } & \multirow[t]{15}{*}{03} & 65 & Retest commit function: & 0 & $\mathrm{x}$ & & & $7 / 09 / 96$ \\
\hline & & & 66 & Redisplay Chemical Component screen: & 0 & $\mathrm{x}$ & & & $7 / 09 / 96$ \\
\hline & & & 67 & Test [Exit] button: & 0 & $\mathrm{x}$ & & & $7 / 09 / 96$ \\
\hline & & & 68 & Load Data Script F17.03-68U & 0 & $\mathrm{x}$ & & & $7 / 09 / 96$ \\
\hline & & & 69 & $\begin{array}{l}\text { Re-enter the LLW Sorting Table screen } \\
\text { (DMSS0312) from the DMS Main Menu; } \\
\text { verify that correct data set is displayed; }\end{array}$ & 0 & $\mathrm{x}$ & & & $7 / 09 / 96$ \\
\hline & & & 70 & Test Sample field for updatability: & 0 & $\mathrm{x}$ & & & $7 / 09 / 96$ \\
\hline & & & 71 & Test Compliant field for enterability: & 0 & $\mathrm{x}$ & & & $7 / 09 / 96$ \\
\hline & & & 72 & $\begin{array}{l}\text { Test [Non-Compliant] button; verify that } \\
\text { correct data set is displayed: }\end{array}$ & 0 & $\mathrm{x}$ & & & $7 / 09 / 96$ \\
\hline & & & 73 & Load Data Script F17.03-73U & 0 & $\mathrm{x}$ & & & $7 / 09 / 96$ \\
\hline & & & 74 & Test [Refresh] button (process update \#5): & 0 & OPEN & & & \\
\hline & & & 75 & Load Data Script F17.03-75U & 0 & & $\mathrm{x}$ & script correction & $7 / 16 / 96$ \\
\hline & & & 76 & Test [Refresh] button (process update \#6): & 0 & & $\mathrm{x}$ & script correction & $7 / 16 / 96$ \\
\hline & & & 77 & $\begin{array}{l}\text { Test [NDE Comment] button; no NDE records } \\
\text { associated with Parent PIN: }\end{array}$ & 0 & $\mathrm{x}$ & & & $7 / 09 / 96$ \\
\hline & & & 78 & Test [Chemcomp] button: & 0 & $\mathrm{x}$ & & & $7 / 09 / 96$ \\
\hline & & & 79 & Re-enter Chemical Component Screen: & 0 & OPEN & & & \\
\hline
\end{tabular}




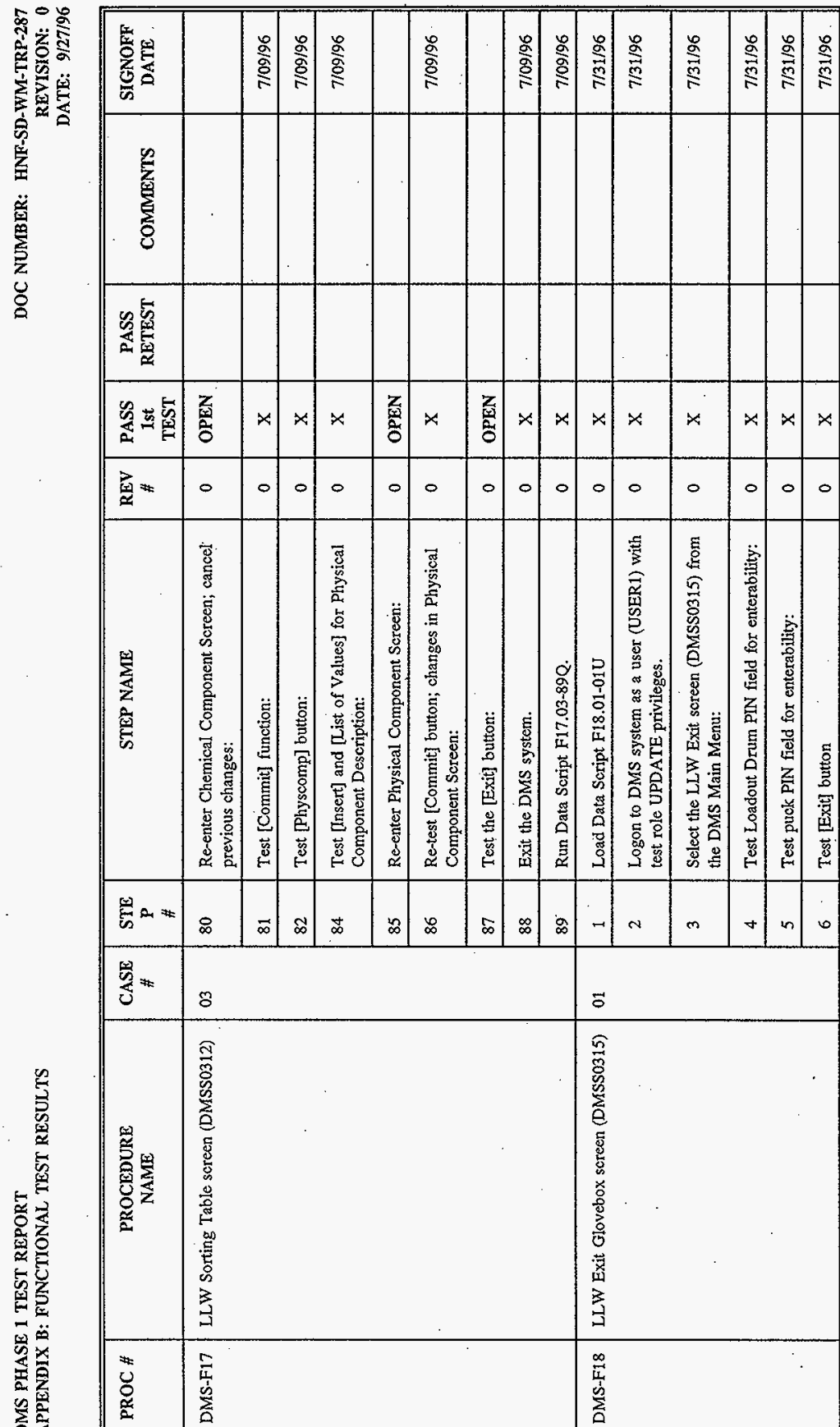


MS PHASE 1 TEST REPORT

APPENDIX B: FUNCTIONAL TEST RESULTS
DOC NUMBER: HNF-SD-WM-TRP-287

REVISION: 0

DATE: $9 / 27 / 96$

\begin{tabular}{|c|c|c|c|c|c|c|c|c|c|}
\hline PROC \# & $\begin{array}{l}\text { PROCEDURE } \\
\text { NAME }\end{array}$ & $\underset{\#}{\text { CASE }}$ & $\begin{array}{c}\text { STE } \\
\mathbf{P} \\
\#\end{array}$ & STEP NAME & $\underset{\#}{\text { REV }}$ & $\begin{array}{c}\text { PASS } \\
\text { 1st } \\
\text { TEST }\end{array}$ & $\begin{array}{l}\text { PASS } \\
\text { RETEST }\end{array}$ & COMMENTS & $\begin{array}{l}\text { SIGNOFF } \\
\text { DATE }\end{array}$ \\
\hline \multirow[t]{15}{*}{ DMS-F18 } & \multirow[t]{15}{*}{ LLW Exit Glovebox screen (DMSS0315) } & 01 & 7 & Exit the DMS system. & 0 & $\mathbf{X}$ & & & $7 / 31 / 96$ \\
\hline & & \multirow[t]{12}{*}{02} & 1 & Run WRESTORE to reinitialize the database. & 0 & $\mathrm{X}$ & & & $7 / 31 / 96$ \\
\hline & & & 2 & $\begin{array}{l}\text { Logon to DMS system as a user (USER1) with } \\
\text { test role UPDATE privileges. }\end{array}$ & 0 & $x$ & & & $7 / 31 / 96$ \\
\hline & & & 3 & $\begin{array}{l}\text { Select the LLW Exit screen (DMSS0315) from } \\
\text { the DMS Main Menu: }\end{array}$ & 0 & OPEN & & & \\
\hline & & & 4 & Test [Exit] button & 0 & $\mathrm{x}$ & & & $7 / 31 / 96$ \\
\hline & & & 5 & Load Data Script F18.02-05U & 0 & & & & $7 / 31 / 96$ \\
\hline & & & 6 & $\begin{array}{l}\text { Select the LLW Exit screen (DMSS0312) from } \\
\text { the DMS Main Menu: }\end{array}$ & 0 & & & & $7 / 31 / 96$ \\
\hline & & & 7 & Test [Exit] button & 0 & & & & $7 / 31 / 96$ \\
\hline & & & 8 & Load Data Script F18.02-08U. & 0 & & & & $7 / 31 / 96$ \\
\hline & & & 9 & $\begin{array}{l}\text { Select the LLW Exit screen (DMSS0315) from } \\
\text { the DMS Main Menu: }\end{array}$ & 0 & . & & & $7 / 31 / 96$ \\
\hline & & & 10 & Test [Exit] button & 0 & & & & $7 / 31 / 96$ \\
\hline & & & 11 & Exit the DMS system. & 0 & & & & $7 / 31 / 96$ \\
\hline & & & 12 & Run post-test query 'f18.02q a'. & 0 & & & & $7 / 31 / 96$ \\
\hline & & \multirow[t]{2}{*}{03} & 1 & Run WRESTORE to reinitialize the database. & 0 & & & & $7 / 31 / 96$ \\
\hline & & & 2 & $\begin{array}{l}\text { Logon to DMS systein as a user (USER1) with } \\
\text { test role UPDATE privileges. }\end{array}$ & 0 & & . & & $7 / 31 / 96$ \\
\hline
\end{tabular}




\begin{tabular}{|c|c|c|c|c|c|c|c|c|c|}
\hline PROC \# & $\begin{array}{l}\text { PROCEDURE } \\
\text { NAME }\end{array}$ & $\underset{\#}{\mathrm{CASE}}$ & $\begin{array}{c}\text { STE } \\
\mathbf{P} \\
\# \\
\end{array}$ & STEP NAME & $\underset{\#}{\mathrm{REV}}$ & $\begin{array}{c}\text { PASS } \\
\text { 1st } \\
\text { TEST }\end{array}$ & $\begin{array}{c}\text { PASS } \\
\text { RETEST }\end{array}$ & COMMENTS & $\begin{array}{l}\text { SIGNOFF } \\
\text { DATE }\end{array}$ \\
\hline \multirow[t]{13}{*}{ DMS-F18 } & \multirow[t]{13}{*}{ LLW Exit Glovebox screen (DMSS0315) } & \multirow[t]{13}{*}{03} & 3 & $\begin{array}{l}\text { Select the LLW Exit Glovebox screen } \\
\text { (DMSS0315) from the DMS Main Menu: }\end{array}$ & 0 & & & & $7 / 31 / 96$ \\
\hline & & & 4 & Test Loadout Drum PIN field for editability: & 0 & & & & $7 / 31 / 96$ \\
\hline & & & 5 & Test tabbing order on screen: & 0 & & & & $7 / 31 / 96$ \\
\hline & & & 6 & Test puck PIN field for enterability: & 0 & & & & $7 / 31 / 96$ \\
\hline & & & 7 & Test puck PIN field for insertability: & 0 & & & & $7 / 31 / 96$ \\
\hline & & & 8 & $\begin{array}{l}\text { Test Generator Waste Description field for } \\
\text { editability, format, length, and case restriction: }\end{array}$ & 0 & & & & $7 / 31 / 96$ \\
\hline & & & 9 & $\begin{array}{l}\text { - Test Estimated Gross Weight (Kg) field for } \\
\text { editability: }\end{array}$ & 0 & & & & $7 / 31 / 96$ \\
\hline & & & 10 & Test Estimated Height (m) field for editability: & 0 & & & & $7 / 31 / 96$ \\
\hline & & & 11 & $\begin{array}{l}\text { Test Primary Waste Type field for editability, } \\
\text { case restriction, format, and length: }\end{array}$ & 0 & & & $\dot{-}$ & $7 / 31 / 96$ \\
\hline & & & 12 & $\begin{array}{l}\text { Test Haz Waste Storage Cat, field for } \\
\text { editability, case restriction, format, and length: }\end{array}$ & 0 & & & . & $7 / 31 / 96$ \\
\hline & & & 13 & $\begin{array}{l}\text { Test Beta-Gamma Dose Rate (mrem } / \mathrm{hr} \text { ) field } \\
\text { for editability, case restriction, and length: }\end{array}$ & 0 & & & & $7 / 31 / 96$ \\
\hline & & & 14 & $\begin{array}{l}\text { Test Seal No. field for editability, case } \\
\text { restriction, format, and length: }\end{array}$ & 0 & & & & $7 / 31 / 96$ \\
\hline & & & 15 & $\begin{array}{l}\text { Test Filler Type field for editability, case } \\
\text { restriction, format, and length: }\end{array}$ & 0 & & & & $7 / 31 / 96$ \\
\hline
\end{tabular}




\begin{tabular}{|c|c|c|c|c|c|c|c|c|c|}
\hline PROC \# & $\begin{array}{l}\text { PROCEDURE } \\
\text { NAME }\end{array}$ & $\underset{\#}{\text { CASE }}$ & $\begin{array}{c}\text { STE } \\
\mathbf{P} \\
\#\end{array}$ & STEP NAME & $\underset{\#}{\text { REV }}$ & $\begin{array}{c}\text { PASS } \\
\text { 1st } \\
\text { TEST }\end{array}$ & $\begin{array}{c}\text { PASS } \\
\text { RETEST }\end{array}$ & COMMENTS & $\begin{array}{l}\text { SIGNOFF } \\
\text { DATE }\end{array}$ \\
\hline \multirow[t]{16}{*}{ DMS-F18 } & \multirow[t]{16}{*}{ LLW Exit Glovebox screen (DMSS0315) } & \multirow[t]{16}{*}{03} & 16 & $\begin{array}{l}\text { Test Filler Weight }(\mathrm{kg}) \text { field for editability, } \\
\text { case restriction, format, and length: }\end{array}$ & 0 & & & & $7 / 31 / 96$ \\
\hline & & & 17 & Load Data Script F18.02-17U. & 0 & & & & $7 / 31 / 96$ \\
\hline & & & 18 & Test [Refresh] button & 0 & & & & $7 / 31 / 96$ \\
\hline & & & 19 & $\begin{array}{l}\text { Test Signature Password and Commit } \\
\text { functions: }\end{array}$ & 0 & & & . & $7 / 31 / 96$ \\
\hline & & & 20 & Retest [Refresh] button: & 0 & OPEN & & & \\
\hline & & & 21 & Test PIN column field for editability: & 0 & & & & $7 / 31 / 96$ \\
\hline & & & 22 & Test Drum PIN column field for editability: & 0 & & & & $7 / 31 / 96$ \\
\hline & & & 23 & $\begin{array}{l}\text { Test Puck Height }(\mathrm{m}) \text { column field for } \\
\text { editability, case restriction, format, and length: }\end{array}$ & 0 & & & & $7 / 31 / 96$ \\
\hline & & & 24 & Receive DMSCOM message F18.03-24U. & 0 & & & & $7 / 31 / 96$ \\
\hline & & & 25 & $\begin{array}{l}\text { Test Puck Weight (kg) column field for } \\
\text { editability: }\end{array}$ & 0 & & & & $7 / 31 / 96$ \\
\hline & & & 26 & Test [Commit] button: & 0 & & & & $7 / 31 / 96$ \\
\hline & & & 27 & Test [Sum Waste Records] button: & 0 & OPEN & & & \\
\hline & & & 28 & Test [Refresh] button: & 0 & OPEN & & & \\
\hline & & & 29 & Test [Sum Waste Records] button: & 0 & OPEN & & & - \\
\hline & & & 30 & Test tabbing sequence for Puck Data block: & 0 & & & & $7 / 31 / 96$ \\
\hline & & & 31 & Test [Sum Waste Records] button: & 0 & OPEN & & & \\
\hline
\end{tabular}




\begin{tabular}{|c|c|c|c|c|c|c|c|c|c|}
\hline PROC \# & $\begin{array}{l}\text { PROCEDURE } \\
\text { NAME }\end{array}$ & $\underset{\#}{\text { CASE }}$ & $\begin{array}{l}\text { STE } \\
\mathbf{P} \\
\#\end{array}$ & STEP NAME & $\stackrel{\text { REV }}{\#}$ & $\begin{array}{l}\text { PASS } \\
\text { 1st } \\
\text { TEST }\end{array}$ & $\begin{array}{c}\text { PASS } \\
\text { RETEST }\end{array}$ & COMMENTS & $\begin{array}{l}\text { SIGNOFF } \\
\text { DATE }\end{array}$ \\
\hline \multirow[t]{16}{*}{ DMS-F18 } & \multirow[t]{16}{*}{ LLW Exit Glovebox screen (DMSS0315) } & \multirow[t]{16}{*}{03} & 32 & Receive DMSCOM message F18.03-30U. & 0 & & & & $7 / 31 / 96$ \\
\hline & & & 33 & Test [Refresh] button: & 0 & OPEN & & & \\
\hline & & & 34 & Receive DMSCOM message F18.03-32U. & 0 & & & & $7 / 31 / 96$ \\
\hline & & & 35 & Test [Sum Waste Records] button: & 0 & OPEN & & & \\
\hline & & & 36 & Test [Refresh] button again: & 0 & OPEN & & & \\
\hline & & & 37 & Test [Commit] button: & 0 & OPEN & & & \\
\hline & & & 38 & Edit Generator Waste Description field: & 0 & OPEN & 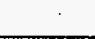 & & \\
\hline & & & 39. & Test [Sum Waste Records] button: & 0 & OPEN & & & \\
\hline & & & 40 & Retest [Sum Waste Records] button: & 0 & OPEN & & & \\
\hline & & & 41 & $\begin{array}{l}\text { Test editability of Puck Height (m) after } \\
\text { performing Sum Waste Records: }\end{array}$ & 0 & & & & $7 / 31 / 96$ \\
\hline & & & 42 & Receive DMSCOM message F18.03-39U. & 0 & & & & $7 / 31 / 96$ \\
\hline & & & 43 & Test [Refresh] button: & 0 & OPEN & & & \\
\hline & & & 44 & Test [Sum Waste Records] button: & 0 & OPEN & & & \\
\hline & & & 45 & Receive DMSCOM message F18.03-42U. & 0 & & & . & $7 / 31 / 96$ \\
\hline & & & 46 & Test [Refresh] button: & 0 & OPEN & & & \\
\hline & & & 47 & $\begin{array}{l}\text { Test }[<] \text { button in Loadout Drum block; } \\
\text { screen displays data for PIN provided by } \\
\text { DMSCOM with location at LLW_EXIT: }\end{array}$ & 0 & & & & $7 / 31 / 96$ \\
\hline
\end{tabular}




\begin{tabular}{|c|c|c|c|c|c|c|c|c|c|}
\hline PROC \# & $\begin{array}{l}\text { PROCEDURE } \\
\text { NAME }\end{array}$ & $\underset{\#}{\text { CASE }}$ & $\begin{array}{c}\text { STE } \\
\text { P } \\
\#\end{array}$ & STEP NAME & $\underset{\#}{\text { REV }}$ & $\begin{array}{l}\text { PASS } \\
\text { 1st } \\
\text { TEST }\end{array}$ & $\begin{array}{l}\text { PASS } \\
\text { RETEST. }\end{array}$ & COMMENTS & $\begin{array}{l}\text { SIGNOFF } \\
\text { DATE }\end{array}$ \\
\hline \multirow[t]{15}{*}{ DMS-F18 } & \multirow[t]{15}{*}{ LLW Exit Glovebox screen (DMSS0315) } & \multirow[t]{15}{*}{03} & 48 & Test $[>]$ button in Puck Data block: & 0 & & & & $7 / 31 / 96$ \\
\hline & & & 49 & $\begin{array}{l}\text { Test scrolling function keys in Puck Data } \\
\text { block: }\end{array}$ & 0 & & & & $7 / 31 / 96$ \\
\hline & & & 50 & Modify Puick Height $(m)$ data values: & 0 & & & & $7 / 31 / 96$ \\
\hline & & & 51 & $\begin{array}{l}\text { Test Next Block function key from Puck Data } \\
\text { block: }\end{array}$ & 0 & & & & $7 / 31 / 96$ \\
\hline & & & 52 & $\begin{array}{l}\text { Test List of Values for Primary Waste Type } \\
\text { field: }\end{array}$ & 0 & & & & $7 / 31 / 96$ \\
\hline & & & 53 & $\begin{array}{l}\text { Test List of Values for Haz. Waste Storage } \\
\text { Cat. field: }\end{array}$ & 0 & & & & $7 / 31 / 96$ \\
\hline & & & 54 & Test List of Values for Filler Type field: & 0 & & & & $7 / 31 / 96$ \\
\hline & & & 55 & Enter Signature Password: & 0 & - & & & $7 / 31 / 96$ \\
\hline & & & 56 & Reccive DMSCOM message F18.03-53U. & 0 & & & v & $7 / 31 / 96$. \\
\hline & & & 57 & Test [Refresh] button: & 0 & OPEN & & & \\
\hline & & & 58 & Retest Refresh function: & 0 & OPEN & & & \\
\hline & & & 59 & Enter simple query to retrieve drum record: & 0 & & & & $7 / 31 / 96$ \\
\hline & & & 60 & Enter missing data values for Loadout Drum: & 0 & & & & $7 / 31 / 96$ \\
\hline & & & 61 & Enter Signature Password: & 0 & & & & $7 / 31 / 96$ \\
\hline & & & 62 & $\begin{array}{l}\text { Enter complex query to retrieve data for } \\
\text { Loadout Drum PINs containing pucks that have } \\
\text { not had waste records summed: }\end{array}$ & 0 & OPEN & & & \\
\hline
\end{tabular}




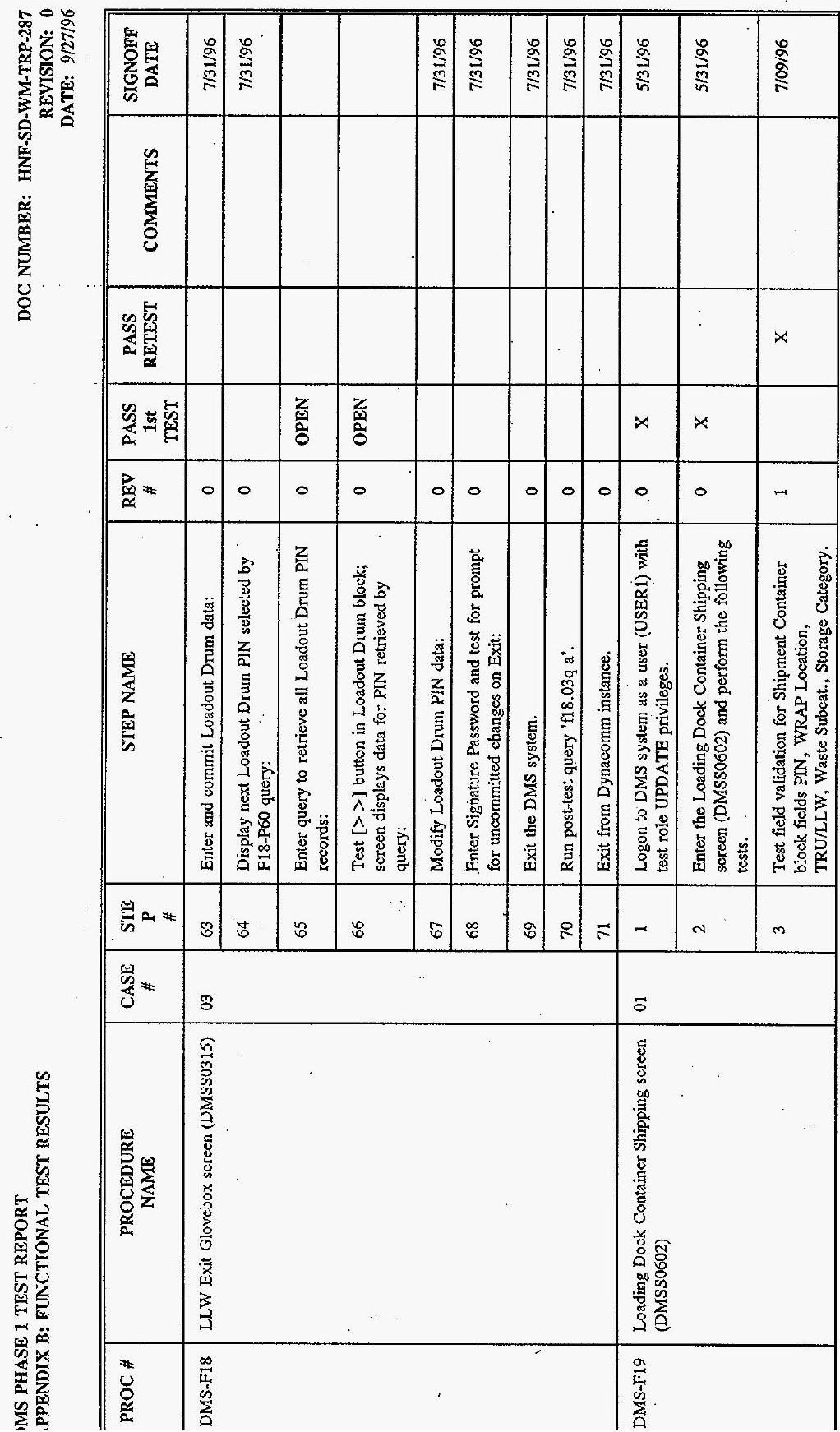




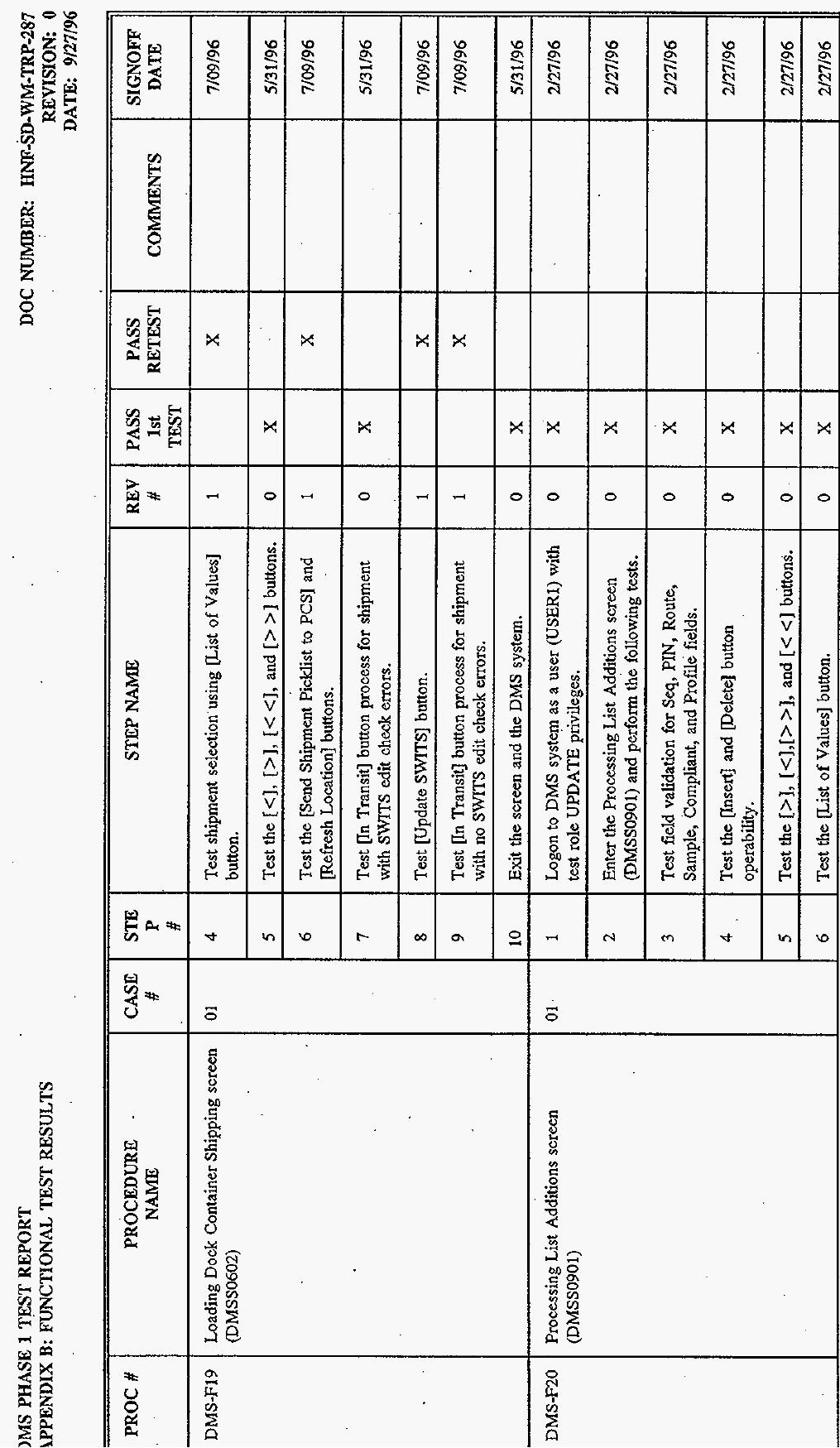




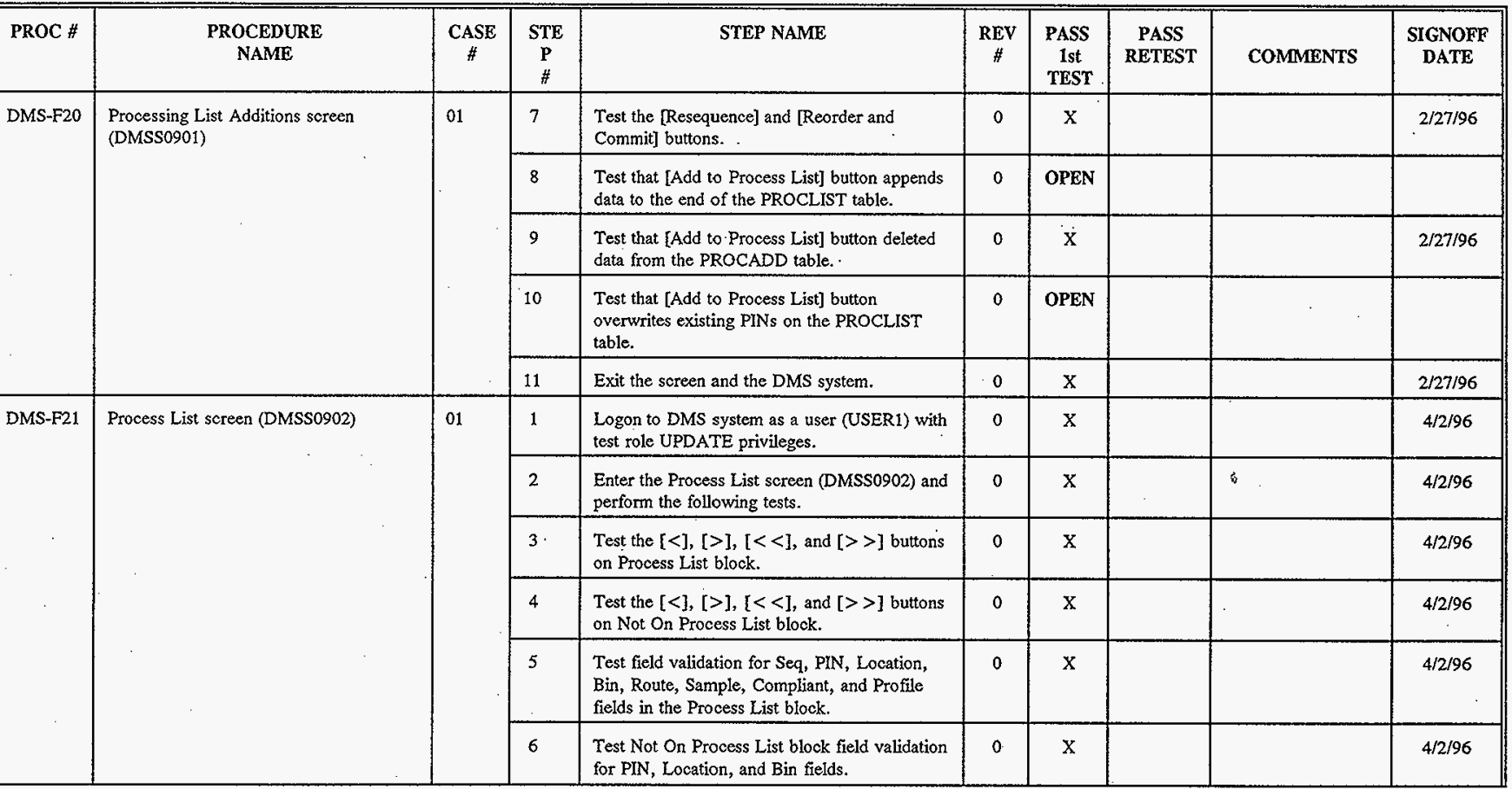


OMS PHASE 1 TEST REPORT

APPENDIX B: FUNCTIONAL TEST RESULTS

DOC NUMBER: HNF-SD-WM-TRP-287

- REVISION: 0

DATE: $9 / 27 / 96$

\begin{tabular}{|c|c|c|c|c|c|c|c|c|c|}
\hline PROC \# & $\begin{array}{l}\text { PROCEDURE } \\
\text { NAME }\end{array}$ & $\underset{\#}{\text { CASE }}$ & $\begin{array}{l}\text { STE } \\
\mathbf{P} \\
\#\end{array}$ & STEP NAME & $\begin{array}{c}\text { REV } \\
\#\end{array}$ & $\begin{array}{l}\text { PASS } \\
\text { 1st } \\
\text { TEST }\end{array}$ & $\begin{array}{c}\text { PASS } \\
\text { RETEST }\end{array}$ & COMMENTS & $\begin{array}{l}\text { SIGNOFF } \\
\text { DATE }\end{array}$ \\
\hline \multirow[t]{7}{*}{ DMS-F21 } & \multirow[t]{7}{*}{ Process List screen (DMSS0902) } & \multirow[t]{7}{*}{01} & 7 & $\begin{array}{l}\text { Test [Exit] and [Delete] buttons and the } \\
\text { signature password field operability }\end{array}$ & 0 & $x$ & & & $4 / 2 / 96$ \\
\hline & & & 8 & Test [List of Values] button operability. & 0 & $\mathrm{X}$ & & & $4 / 2 / 96$ \\
\hline & & & 9 & Test [Move] button operability. & 0 & $\mathrm{x}$ & & & $4 / 2 / 96$ \\
\hline & & & 10 & $\begin{array}{l}\text { Test [Resequence] and [Reorder and Commit] } \\
\text { button operability. }\end{array}$ & 0 & $\mathrm{x}$ & & & $4 / 2 / 96$ \\
\hline & & & 11 & Test Pick List Selection. & 0 & $\mathrm{X}$ & & & $4 / 2 / 96$ \\
\hline & & & 12 & Test [Send Process Pick List to PCS] button. & 1 & & $\mathrm{x}$ & & $6 / 6 / 96$ \\
\hline & & & 13 & Exit the screen and the DMS system. & 0 & $\mathrm{X}$ & & & $4 / 2 / 96$ \\
\hline \multirow[t]{6}{*}{ DMS-F22 } & \multirow{6}{*}{$\begin{array}{l}\text { AS/RS Storage Pick List screen } \\
\text { (DMSS0903) } \\
\end{array}$} & \multirow[t]{6}{*}{01} & 1 & $\begin{array}{l}\text { Logon to DMS system as a user (USER1) with } \\
\text { test role UPDATE privileges. }\end{array}$ & 0 & $x$ & & & $4 / 8 / 96$ \\
\hline & & & 2 & $\begin{array}{l}\text { Enter the AS/RS Storage Pick List screen } \\
\text { (DMSS0903) and perform the following tests. }\end{array}$ & 0 & $x$ & & & $4 / 8 / 96$ \\
\hline & & & 3 & $\begin{array}{l}\text { Test the }[<],[>],[<<] \text {, and }[>>] \text { buttons } \\
\text { on AS/RS Storage Pick List block. }\end{array}$ & 0 & $x$ & & & $4 / 8 / 96$ \\
\hline & & & 4 & $\begin{array}{l}\text { Test AS/RS Storage Pick List block field } \\
\text { validation for Bin, PIN, Waste Type, LLW } \\
\text { category, and Storage Code }\end{array}$ & 0 & $x$ & & & $4 / 8 / 96$ \\
\hline & & & 5 & $\begin{array}{l}\text { Test Discharge Conveyor block field validation } \\
\text { for PIN, Waste Type, LLW category, and } \\
\text { Storage Code }\end{array}$ & 0 & $x$ & . & & $4 / 8 / 96$ \\
\hline & & & 6 & Test [Exit] button operability & 0 & $\mathbf{x}$ & & & $4 / 8 / 96$ \\
\hline
\end{tabular}




\begin{tabular}{|c|c|c|c|c|c|c|c|c|c|}
\hline PROC \# & $\begin{array}{l}\text { PROCEDURE } \\
\text { NAME }\end{array}$ & $\underset{\#}{\text { CASE }}$ & $\begin{array}{c}\text { STE } \\
\mathbf{P} \\
\sharp\end{array}$ & STEP NAME & $\stackrel{\text { REV }}{\#}$ & $\begin{array}{l}\text { PASS } \\
\text { 1st } \\
\text { TEST }\end{array}$ & $\begin{array}{l}\text { PASS } \\
\text { RETEST }\end{array}$ & COMMENTS & $\begin{array}{l}\text { SIGNOFF } \\
\text { DATE }\end{array}$ \\
\hline \multirow[t]{4}{*}{ DMS-F22 } & \multirow{4}{*}{$\begin{array}{l}\text { AS/RS Storage Pick List screen } \\
\text { (DMSS0903) }\end{array}$} & \multirow[t]{4}{*}{01} & 7 & Test default display type & 0 & $\mathrm{x}$ & & $\cdot$ & $4 / 8 / 96$ \\
\hline & & & 8 & Test [LLW] and [TRU] buttons & 0 & $\mathrm{x}$ & & & $4 / 8 / 96$ \\
\hline & & & 9 & Test [Retrieve PIN] button. & 1 & & $\mathrm{x}$ & & $6 / 6 / 96$ \\
\hline & & & 10 & Exit the screen and the DMS system. & 0 & $\mathrm{x}$ & & & $4 / 8 / 96$ \\
\hline \multirow[t]{9}{*}{ DMS-F23 } & \multirow[t]{9}{*}{$\begin{array}{l}\text { AS/RS Shipping Pick List screen } \\
\text { (DMSS0904) }\end{array}$} & \multirow[t]{9}{*}{01} & 1 & $\begin{array}{l}\text { Logon to DMS system as a user (USER1) with } \\
\text { test role UPDATE privileges. }\end{array}$ & 1 & $x$ & & & $5 / 31 / 96$ \\
\hline & & & 2 & $\begin{array}{l}\text { Enter the AS/RS Shipping Pick List screen } \\
\text { (DMSS0904) and perform the following tests. }\end{array}$ & 1 & $\mathrm{x}$ & & & $5 / 31 / 96$ \\
\hline & & & 3 & $\begin{array}{l}\text { Test Data fields on both left and right sides of } \\
\text { the screen to ensure that data is not manually } \\
\text { enterable in any fields. }\end{array}$ & 1 & & $\mathrm{x}$ & & $6 / 5 / 96$ \\
\hline & & & 4 & Test [LLW] and [TRU] buttons. & 1 & $\mathrm{x}$ & & & $5 / 31 / 96$ \\
\hline & & & 5 & $\begin{array}{l}\text { Test the }[<],[>],[<<] \text {, and }[>>] \text { buttons } \\
\text { on Right side of sereen. }\end{array}$ & 1 & $\mathrm{x}$ & & & $5 / 31 / 96$ \\
\hline & & & 6 & $\begin{array}{l}\text { Test [Clear Form], [Move], and [Commit] } \\
\text { buttons. }\end{array}$ & 1 & $\mathrm{x}$ & & & $5 / 31 / 96$ \\
\hline & & & 7. & $\begin{array}{l}\text { Test [LOV] button, shipment \# popup, and } \\
\text { [Commit] button. }\end{array}$ & 1 & $\mathrm{x}$ & & & $5 / 31 / 96$ \\
\hline & & & 8 & $\begin{array}{l}\text { Test the }[<],[>],[<<] \text {, and }[>>] \text { buttons } \\
\text { on Left side of screen. }\end{array}$ & 1 & $x$ & & & $5 / 31 / 96$ \\
\hline & & & 9 & Exit the screen and the DMS system. & 1 & $\mathrm{x}$ & & & $5 / 31 / 96$ \\
\hline
\end{tabular}




\begin{tabular}{|c|c|c|c|c|c|c|c|c|c|}
\hline PROC \# & $\begin{array}{l}\text { PROCEDURE } \\
\text { NAME }\end{array}$ & $\underset{\#}{\text { CASE }}$ & $\begin{array}{c}\text { SrE } \\
\mathbf{p} \\
\#\end{array}$ & STEP NAME & $\underset{\#}{\mathrm{REV}}$ & $\begin{array}{l}\text { PASS } \\
\text { 1st } \\
\text { TEST }\end{array}$ & $\begin{array}{c}\text { PASS } \\
\text { RETEST }\end{array}$ & COMMENTS & $\begin{array}{l}\text { SIGNOFF } \\
\text { DATE }\end{array}$ \\
\hline \multirow[t]{6}{*}{ DMS-F24 } & \multirow[t]{6}{*}{$\begin{array}{l}\text { Radiological Inventory Summary screen } \\
\text { (DMSS1101) }\end{array}$} & \multirow[t]{6}{*}{01} & 1 & $\begin{array}{l}\text { Logon to DMS system as a user (USER1) with } \\
\text { test role UPDATE privileges. }\end{array}$ & 0 & $\mathrm{x}$ & & & $3 / 1 / 96$ \\
\hline & & & 2 & $\begin{array}{l}\text { Enter the Radiological Inventory Summary } \\
\text { screen (DMSS1 101) and perform the following } \\
\text { tests. }\end{array}$ & 0 & $\mathrm{x}$ & & & $3 / 1 / 96$ \\
\hline & & & 4 & $\begin{array}{l}\text { Test the effects of changing the value in the } \\
\text { Total field. }\end{array}$ & 1 & & $\mathrm{x}$ & & $3 / 13 / 96$ \\
\hline & & & 5 & Test the [Exit] button. & 0 & $\mathrm{x}$ & & & $3 / 1 / 96$ \\
\hline & & & 6 & Test the [Print] button. & 0 & $\mathrm{x}$ & & & $3 / 1 / 96$ \\
\hline & & & 7 & Exit screen and DMS system. & 0 & $\mathrm{x}$ & & & $3 / 1 / 96$ \\
\hline \multirow[t]{5}{*}{ DMS-F25 } & \multirow{5}{*}{$\begin{array}{l}\text { Processed Waste Data Review \& Mod. } \\
\text { screen (DMSS1221) }\end{array}$} & \multirow[t]{5}{*}{01} & 1 & Load Data Script F25.01-01U & 0 & $\mathrm{x}$ & & & $7 / 22 / 96$ \\
\hline & & & 3 & $\begin{array}{l}\text { Select the Processed Waste Data Review \& } \\
\text { Mod. screen (DMSS1221) from the DMS Main } \\
\text { Menu: }\end{array}$ & 0 & $\mathrm{x}$ & & & $7 / 22 / 96$ \\
\hline & & & 4 & Test [Waste Packet Desig.] button: & 0 & $\mathrm{x}$ & & & $7 / 22 / 96$ \\
\hline & & & 5 & Test [Perform Data Review] button: & 0 & $\mathrm{x}$ & & & $7 / 22 / 96$ \\
\hline & & & 6 & Test [Data Review Complete] button: & 0 & $\mathrm{x}$ & & & $7 / 22 / 96$ \\
\hline
\end{tabular}




\begin{tabular}{|c|c|c|c|c|c|c|c|c|c|}
\hline PROC \# & $\begin{array}{l}\text { PROCEDURE } \\
\text { NAME }\end{array}$ & $\underset{\#}{\mathrm{CASE}}$ & $\begin{array}{c}\text { STE } \\
\mathbf{P} \\
\# \\
\end{array}$ & STEP NAM̄E & $\underset{\#}{\text { REV }}$ & $\begin{array}{l}\text { PASS } \\
\text { 1st } \\
\text { TEST }\end{array}$ & $\begin{array}{l}\text { PASS } \\
\text { RETEST }\end{array}$ & COMMENTS & $\begin{array}{l}\text { SIGNOFF } \\
\text { DATE }\end{array}$ \\
\hline \multirow[t]{14}{*}{ DMS-F25 } & \multirow{14}{*}{$\begin{array}{l}\text { Processed Waste Data Review \& Mod. } \\
\text { screen (DMSS1221) }\end{array}$} & \multirow[t]{14}{*}{01} & 7 & Test [WASTE] button: & 0 & $\mathrm{x}$ & & & $7 / 22 / 96$ \\
\hline & & & 8 & Test [RADDETAIL] button: & 0 & $\mathrm{X}$ & & & $7 / 22 / 96$ \\
\hline & & & 9 & Test [SSOQTY] button: & 0 & $\mathrm{x}$ & & & $7 / 22 / 96$ \\
\hline & & & 10 & Test [PHYSCOMP] button: & 0 & $\mathrm{x}$ & & & $7 / 22 / 96$ \\
\hline & & & 11 & Test [HAZDETAUL] button: & 1 & & $\mathrm{x}$ & & $8 / 14 / 96$ \\
\hline & & & 12 & Test [CHEMCOMP] button: & 0 & $\mathrm{x}$ & & & $7 / 22 / 96$ \\
\hline & & & 13 & Test [APPMSDS] button: & 0 & $\mathrm{x}$ & & & $7 / 22 / 96$ \\
\hline & & & 14 & Test [CONREL] button: & 0 & $\mathrm{x}$ & & & $7 / 22 / 96$ \\
\hline & & & 15 & $\begin{array}{l}\text { Test Drum PIN field for required entry/tabbing } \\
\text { sequence: }\end{array}$ & 0 & $\mathrm{x}$ & & & $7 / 22 / 96$ \\
\hline & & & 16 & $\begin{array}{l}\text { Test Drum PIN field for updatability, case } \\
\text { restriction, format, length, and valid value; test } \\
\text { [Perform Data Review] button: }\end{array}$ & 0 & $x$ & & & $7 / 22 / 96$ \\
\hline & & & 17 & $\begin{array}{l}\text { Test [list of Values] button - default selection } \\
\text { criteria ('LLW' and 'Not Reviewed'): }\end{array}$ & 0 & $\dot{x}$ & & & $7 / 22 / 96$ \\
\hline & & & 18 & $\begin{array}{l}\text { Test waste type selection criteria; test [List of } \\
\text { Values] button for 'TRU' and 'Not Reviewed': }\end{array}$ & 0 & $\mathrm{x}$. & & & $7 / 22 / 96$ \\
\hline & & & 19 & Test [Perform Data Review] button: & 0 & $\mathrm{x}$ & & & $7 / 22 / 96$ \\
\hline & & & 20 & $\begin{array}{l}\text { Test waste type selection criteria; test [List of } \\
\text { Values] button for 'All' and 'Not Reviewed': }\end{array}$ & 0 & $\mathrm{x}$ & & & $7 / 22 / 96$ \\
\hline
\end{tabular}




\begin{tabular}{|c|c|c|c|c|c|c|c|c|c|}
\hline PROC \# & $\begin{array}{l}\text { PROCEDURE } \\
\text { NAME }\end{array}$ & $\underset{\#}{\mathrm{CASE}}$ & $\begin{array}{c}\text { STE } \\
P \\
\# \\
\end{array}$ & STEP NAME & $\underset{\#}{\text { REV }}$ & $\begin{array}{c}\text { PASS } \\
\text { 1st } \\
\text { TEST }\end{array}$ & $\begin{array}{c}\text { PASS } \\
\text { RETEST }\end{array}$ & COMMENTS & $\begin{array}{l}\text { SIGNOFF } \\
\text { DATE }\end{array}$ \\
\hline \multirow[t]{16}{*}{ DMS-F25 } & \multirow{16}{*}{$\begin{array}{l}\text { Processed Waste Data Review \& Mod. } \\
\text { screen (DMSS1221) }\end{array}$} & \multirow[t]{11}{*}{02} & 9 & Test [WASTE] button: & 0 & $\mathrm{x}$ & & & $7 / 22 / 96$ \\
\hline & & & 10 & Test [RADDETAIL] button: & 0 & $\mathrm{x}$ & & & $7 / 22 / 96$ \\
\hline & & & 11 & Test [ISOQTY] button: & 0 & $\mathrm{x}$ & & & $7 / 22 / 96$ \\
\hline & & & 12 & Test [PHYSCOMP] button: & 1 & & $\mathrm{x}$ & . & $8 / 14 / 96$ \\
\hline & & & 13 & Test [HAZDETAIL] button: & 0 & $x$ & & & $7 / 22 / 96$ \\
\hline & & & 14 & Teșt [CHEMCOMP] button: & 0 & $\mathrm{x}$ & & & $7 / 22 / 96$ \\
\hline & & & 15 & Test [APPMSDS] button: & 0 & $x$ & & & $7 / 22 / 96$ \\
\hline & & & 16 & Test [CONREL] button: & 0 & $\mathrm{x}$ & & & $7 / 22 / 96$ \\
\hline & & & 17 & Test [Exit] button: & 0 & $x$ & & & $7 / 22 / 96$ \\
\hline & & & 18 & Exit the DMS system. & 0 & $x$ & & & $7 / 22 / 96$ \\
\hline & & & 19 & Run post-test query ' $225.02 q a^{\prime}$ '. & 0 & $x$ & & & $7 / 22 / 96$ \\
\hline & & \multirow[t]{5}{*}{03} & 1 & Run pre-test query ' $25.03 \mathrm{q}$ b'. & 0 & $\mathrm{x}$ & & & $7 / 22 / 96$ \\
\hline & & & 2 & $\begin{array}{l}\text { Logon to DMS system as a user (USER1) with } \\
\text { test role UPDATE privileges. }\end{array}$ & 0 & $\mathrm{x}$ & & & $7 / 22 / 96$ \\
\hline & & & 3 & $\begin{array}{l}\text { Select the Processed Waste Data Review \& } \\
\text { Mod. screen (DMSS1221) from the DMS Main } \\
\text { Menu: }\end{array}$ & 0 & $x$ & & & $7 / 22 / 96$ \\
\hline & & & 4 & Enter a PIN for review: & 0 & $\mathrm{x}$ & & & $7 / 22 / 96$ \\
\hline & & & 5 & Test [Data Review Complete] button: & 1 & & $\mathbf{x}$ & & $8 / 14 / 96$ \\
\hline
\end{tabular}




\begin{tabular}{|c|c|c|c|c|c|c|c|c|c|}
\hline PROC \# & $\begin{array}{l}\text { PROCEDURE } \\
\text { NAME }\end{array}$ & $\underset{\#}{\text { CASE }}$ & $\begin{array}{c}\text { STE } \\
\mathbf{p} \\
\#\end{array}$ & STEP NAME & $\underset{\#}{\mathrm{REV}}$ & $\begin{array}{c}\text { PASS } \\
\text { 1st } \\
\text { TEST }\end{array}$ & $\begin{array}{c}\text { PASS } \\
\text { RETEST }\end{array}$ & COMMENTS & $\begin{array}{l}\text { SIGNOFF } \\
\text { DATE }\end{array}$ \\
\hline \multirow[t]{18}{*}{ DMS-F25 } & \multirow{18}{*}{$\begin{array}{l}\text { Processed Waste Data Review \& Mod. } \\
\text { screen (DMSS1221) }\end{array}$} & \multirow[t]{18}{*}{03} & 6 & Test [WASTE] button: & 0 & $x$ & & & $7 / 22 / 96$ \\
\hline & & & 7 & Test [RADDETAIL] button: & 1 & & $\mathrm{x}$ & & $8 / 14 / 96$ \\
\hline & & & 8 & Test [ISOQTY] button: & 1 & & $\mathrm{x}$ & & $8 / 14 / 96$ \\
\hline & & & 9 & Test [PHYSCOMPI button: & 0 & $\mathrm{X}$ & & & $7 / 22 / 96$ \\
\hline & & & 10 & Test [HAZDETAIL] button: & 0 & $\mathrm{x}$ & & & $7 / 22 / 96$ \\
\hline & & & 11 & Test [CHEMCOMP] button: & 0 & $\mathrm{x}$ & & & $7 / 22 / 96$ \\
\hline & & & 12 & Test [APPMSDS] button: & 0 & $\mathrm{X}$ & & & $7 / 22 / 96$ \\
\hline & & & 13 & Test [CONREL] button: & 0 & $\mathrm{x}$ & & & $7 / 22 / 96$ \\
\hline & & & 14 & Retest [ISOQTY] button: & 0 & $\mathrm{x}$ & & & $7 / 22 / 96$ \\
\hline & & & 15 & Test [Perform Data Review] button: & 0 & $x$ & 4 & & $7 / 22 / 96$ \\
\hline & & & 16 & Enter PIN for review: & 0 & $\mathrm{x}$ & . & & $7 / 22 / 96$ \\
\hline & & & 17 & Test [Perform Data Review] button: & 0 & $\mathrm{x}$ & & & $7 / 22 / 96$ \\
\hline & & & 18 & Test [WASTE] button: & 0 & $x$ & & & $7 / 22 / 96$ \\
\hline & & & 19 & Test [RADDETAIL] button: & 0 & $\mathrm{x}$ & & & $7 / 22 / 96$ \\
\hline & & & 20 & Test [SOQTY] button: & 0 & $\mathrm{x}$ & & & $7 / 22 / 96$ \\
\hline & & & 21 & Test [PHYSCOMP] button: & 0 & $\mathrm{x}$ & & & $7 / 22 / 96$ \\
\hline & & & 22 & Test [HAZDETAIL] button: & 0 & $\mathrm{x}$ & & & $7 / 22 / 96$ \\
\hline & & & 23 & Test [CHEMCOMP] button: & 0 & $x$ & & & $7 / 22 / 96$ \\
\hline
\end{tabular}




\begin{tabular}{|c|c|c|c|c|c|c|c|c|c|}
\hline PROC \# & $\begin{array}{l}\text { PROCEDURE } \\
\text { NAME }\end{array}$ & $\underset{\#}{\text { CASE }}$ & $\begin{array}{l}\text { STE } \\
\mathbf{P} \\
\# \\
\end{array}$ & STEP NAME & $\underset{\#}{\mathbf{R E V}}$ & $\begin{array}{l}\text { PASS } \\
\text { 1st } \\
\text { TEST }\end{array}$ & $\begin{array}{c}\text { PASS } \\
\text { RETEST }\end{array}$ & COMMENTS & $\begin{array}{l}\text { SIGNOFF } \\
\text { DATE }\end{array}$ \\
\hline \multirow[t]{13}{*}{ DMS-F25 } & \multirow[t]{13}{*}{$\begin{array}{l}\text { Processed Waste Data Review \& Mod. } \\
\text { screen (DMSS1221) }\end{array}$} & \multirow[t]{13}{*}{04} & 3 & $\begin{array}{l}\text { Select the Processed Waste Data Review \& } \\
\text { Mod. screen (DMSS1221) from the DMS Main } \\
\text { Menu: }\end{array}$ & 0 & $\mathrm{x}$ & & & $7 / 22 / 96$ \\
\hline & & & 4 & Test [List of Values] button for drum PIN: & 0 & $\mathrm{x}$ & & & $7 / 22 / 96$ \\
\hline & & & 5 & Test [Perform Data Review] button: & 0 & $\mathrm{x}$ & & & $7 / 22 / 96$ \\
\hline & & & 6 & $\begin{array}{l}\text { Test [Commit] button for Certification Data } \\
\text { Error Summary: }\end{array}$ & 0 & $\mathrm{x}$ & & & $7 / 22 / 96$ \\
\hline & & & 7 & $\begin{array}{l}\text { Test down function key for Certification Data } \\
\text { Error Summary: }\end{array}$ & 0 & $\mathrm{x}$ & & & $7 / 22 / 96$ \\
\hline & & & 8 & $\begin{array}{l}\text { Test }[<] \text { and }[>] \text { buttons for Certification } \\
\text { Data Error Summary: }\end{array}$ & 0 & $\mathrm{x}$ & & & $7 / 22 / 96$ \\
\hline & & & 9 & Test [WASTE] button: & 0 & $\mathrm{x}$ & & & $7 / 22 / 96$ \\
\hline & & & 10 & Test screen for record scrolling: & 0 & $\mathrm{x}$ & & & $7 / 22 / 96$ \\
\hline & & & 11 & Test delete record function: & 0 & $\mathrm{x}$ & & & $7 / 22 / 96$ \\
\hline & & & 12 & $\begin{array}{l}\text { Test Primary Waste Type field for length, } \\
\text { format, and case restriction: }\end{array}$ & 0 & $\mathrm{x}$ & & & $7 / 22 / 96$ \\
\hline & & & 13 & $\begin{array}{l}\text { Test Chemical Nature field for length, format, } \\
\text { and case restriction: }\end{array}$ & 0 & $\mathrm{x}$ & & & $7 / 22 / 96$ \\
\hline & & & 14 & $\begin{array}{l}\text { Test Storage Category field for length, format, } \\
\text { and case restriction: }\end{array}$ & 0 & $\mathrm{x}$ & & & $7 / 22 / 96$ \\
\hline & & & 15 & $\begin{array}{l}\text { Test Package Status field for length, format, } \\
\text { and case restriction: }\end{array}$ & 0 & $x$ & & & $7 / 22 / 96$ \\
\hline
\end{tabular}




\begin{tabular}{|c|c|c|c|c|c|c|c|c|c|}
\hline PROC \# & $\begin{array}{l}\text { PROCEDURE } \\
\text { NAME }\end{array}$ & $\underset{\#}{\text { CASE }}$ & $\begin{array}{c}\text { STE } \\
\mathbf{P} \\
\# \\
\end{array}$ & STEP NAME & $\underset{H}{\mathrm{REV}}$ & $\begin{array}{c}\text { PASS } \\
\text { 1st } \\
\text { TEST } \\
\end{array}$ & $\begin{array}{c}\text { PASS } \\
\text { RETEST }\end{array}$ & COMMENTS & $\begin{array}{l}\text { SIGNOFF } \\
\text { DATE }\end{array}$ \\
\hline \multirow[t]{14}{*}{ DMS-F25 } & \multirow[t]{14}{*}{$\begin{array}{l}\text { Processed Waste Data Review \& Mod. } \\
\text { screen (DMSS1221) }\end{array}$} & \multirow[t]{14}{*}{04} & 16 & $\begin{array}{l}\text { Test Labpack Flag field for length, format, and } \\
\text { case restriction: }\end{array}$ & 1 & & $\mathrm{x}$ & & $8 / 14 / 96$ \\
\hline & & & 17 & $\begin{array}{l}\text { Test Routine field for length, format, and case } \\
\text { restriction: }\end{array}$ & 0 & $\mathrm{x}$ & & & $7 / 22 / 96$ \\
\hline & & & 18 & Test Gross Weight (kg) field for updatability: & 0 & $x$ & & & $7 / 22 / 96$ \\
\hline & & & 19 & Test Waste Weight $(\mathrm{kg})$ field for updatability: & 0 & $\mathrm{x}$ & & & $7 / 22 / 96$ \\
\hline & & & 20 & Test Secondary PIN field for updatability: & 0 & $\mathrm{x}$ & & & $7 / 22 / 96$ \\
\hline & & & 21 & $\begin{array}{l}\text { Test Generator Comment field for length, } \\
\text { format, and case restriction: }\end{array}$ & 0 & $x$ & & & $7 / 22 / 96$ \\
\hline & & & 22 & $\begin{array}{l}\text { Test Waste Description field for Autohint and } \\
\text { updatability: }\end{array}$ & 0 & $\mathrm{x}$ & & & $7 / 22 / 96$ \\
\hline & & & 23 & $\begin{array}{l}\text { Test Accumulation Date field for format, } \\
\text { length, and case restriction: }\end{array}$ & 1 & · & $\mathrm{x}$ & & $8 / 20 / 96$ \\
\hline & & & 24 & $\begin{array}{l}\text { Test Package Date field for format, length, and } \\
\text { case restriction: }\end{array}$ & 0 & $\mathrm{x}$ & & & $7 / 22 / 96$ \\
\hline & & & 25 & $\begin{array}{l}\text { Test Treatment Date field for format, length, } \\
\text { and case restriction: }\end{array}$ & 0 & $\mathrm{x}$ & & & $7 / 22 / 96$ \\
\hline & & & 26 & Test TSD Accept Date field for updatability: & 0 & $\mathrm{x}$ & & & $7 / 22 / 96$ \\
\hline & & & 27 & Test Source Company ID field for updatability: & 0 & $x$ & & & $7 / 22 / 96$ \\
\hline & & & 28 & Test Source Facility ID field for updatability: & 0 & $x$ & & & $7 / 22 / 96$. \\
\hline & & & 29 & Test [Return to Main 1221 Screen] button: & 0 & $\mathrm{x}$ & & & $7 / 22 / 96$ \\
\hline
\end{tabular}




\begin{tabular}{|c|c|c|c|c|c|c|c|c|c|}
\hline PROC \# & $\begin{array}{l}\text { PROCEDURE } \\
\text { NAME }\end{array}$ & $\underset{\#}{\text { CASE }}$ & $\begin{array}{c}\text { STE } \\
\mathbf{P} \\
\# \\
\end{array}$ & STEP NAME & $\begin{array}{c}\text { REV } \\
\# \\
\end{array}$ & $\begin{array}{l}\text { PASS } \\
\text { 1st } \\
\text { TEST }\end{array}$ & $\begin{array}{c}\text { PASS } \\
\text { RETEST }\end{array}$ & COMMENTS & $\begin{array}{l}\text { SIGNOFF } \\
\text { DATE }\end{array}$ \\
\hline \multirow[t]{13}{*}{ DMS-F25 } & \multirow{13}{*}{$\begin{array}{l}\text { Processed Waste Data Review \& Mod. } \\
\text { screen (DMSS1221) }\end{array}$} & \multirow[t]{13}{*}{04} & 30 & Test [Perform Data Review] button: & 0 & $\mathrm{x}$ & & & $7 / 22 / 96$ \\
\hline & & & 31 & Test [RADDETAIL] button: & 0 & $\mathrm{x}$ & & & $7 / 22 / 96$ \\
\hline & & & 32 & Test screen for record scrolling: & 0 & $\mathrm{x}$ & & & $7 / 22 / 96$ \\
\hline & & & 33 & Test delete record function: & 0 & $\mathrm{x}$ & & . & $7 / 22 / 96^{\circ}$ \\
\hline & & & 34 & $\begin{array}{l}\text { Test Secondary Waste Type field for length, } \\
\text { format, and case restriction: }\end{array}$ & 0 & $\mathrm{x}$ & & & $7 / 22 / 96$ \\
\hline & & & 35 & $\begin{array}{l}\text { Test Sec. Waste Type Group field for Autohint } \\
\text { and updatability: }\end{array}$ & 0 & $\mathrm{x}$ & & & $7 / 22 / 96$ \\
\hline & & & 36 & $\begin{array}{l}\text { Test Content Thermal Power field for case } \\
\text { restriction: }\end{array}$ & 1 & & $\mathrm{x}$ & & $8 / 20 / 96$ \\
\hline & & & 37 & $\begin{array}{l}\text { Test Seal Number field for format, length, and } \\
\text { case restriction: }\end{array}$ & 0 & $\mathrm{x}$ & & & $7 / 22 / 96$ \\
\hline & & & 38 & $\begin{array}{l}\text { Test Assay Number field for format, length, } \\
\text { and case restriction: }\end{array}$ & 0 & $\mathrm{x}$ & & & $7 / 22 / 96$ \\
\hline & & & 39 & $\begin{array}{l}\text { Test Assay Date field for format, length, and } \\
\text { case restrictions: }\end{array}$ & 0 & $\mathrm{x}$ & & & $7 / 22 / 96$ \\
\hline & & & 40 & $\begin{array}{l}\text { Test Waste Category field for format, length, } \\
\text { and case restriction: }\end{array}$ & 0 & $\mathrm{x}$ & & & $7 / 22 / 96$ \\
\hline & & & 41 & $\begin{array}{l}\text { Test Waste Makeup field for format, length, } \\
\text { and case restriction: }\end{array}$ & 0 & $\mathrm{x}$ & & & $7 / 22 / 96$ \\
\hline & & & 42 & $\begin{array}{l}\text { Test Contact/Remote Handling field for } \\
\text { format, length, and case restriction: }\end{array}$ & 0 & $\mathbf{x}$ & & & $7 / 22 / 96$ \\
\hline
\end{tabular}




\begin{tabular}{|c|c|c|c|c|c|c|c|c|c|}
\hline PROC \# & $\begin{array}{l}\text { PROCEDURE } \\
\text { NAME }\end{array}$ & $\underset{\#}{\operatorname{CASE}}$ & $\begin{array}{c}\text { STE } \\
\mathbf{P} \\
\# \\
\end{array}$ & STEP NAME & $\underset{\#}{\text { REV }}$ & $\begin{array}{c}\text { PASS } \\
\text { 1st } \\
\text { TEST }\end{array}$ & $\begin{array}{l}\text { PASS } \\
\text { RETEST }\end{array}$ & COMMENTS & $\begin{array}{l}\text { SIGNOFF } \\
\text { DATE }\end{array}$ \\
\hline \multirow[t]{11}{*}{ DMS-F25 } & \multirow[t]{11}{*}{$\begin{array}{l}\text { Processed Waste Data Review \& Mod. } \\
\text { screen (DMSS1221) }\end{array}$} & \multirow[t]{11}{*}{04} & 43 & $\begin{array}{l}\text { Test Void Space Filler field for format, length, } \\
\text { and case restriction: }\end{array}$ & 0 & $\mathrm{x}$ & & & $7 / 22 / 96$ \\
\hline & & & 44 & $\begin{array}{l}\text { Test Void Space Filler Descr. field for } \\
\text { Autohint and updatability: }\end{array}$ & 1 & & $\mathrm{x}$ & & $8 / 14 / 96$ \\
\hline & & & 45 & $\begin{array}{l}\text { Test SWIMS Category field for format, length, } \\
\text { and case restriction: }\end{array}$ & 0 & $\mathrm{x}$ & & & $7 / 22 / 96$ \\
\hline & & & 46 & $\begin{array}{l}\text { Test SWMS Category Deser. field for } \\
\text { Authohint and updatability: }\end{array}$ & 1 & & $\mathrm{x}$ & & $8 / 14 / 96$ \\
\hline & & & 47 & $\begin{array}{l}\text { Test WRAP Category field for length, format, } \\
\text { and case restriction: }\end{array}$ & 0 & $\mathrm{x}$ & & & $7 / 22 / 96$ \\
\hline & & & 48 & $\begin{array}{l}\text { Test WRAP Category Descr. for Autohint and } \\
\text { updatability: }\end{array}$ & 1 & & $\mathrm{x}$ & & $8 / 14 / 96$ \\
\hline & & & 49 & $\begin{array}{l}\text { Test RSWIMS Container Count field for } \\
\text { updatability: }\end{array}$ & 0 & $\mathrm{x}$ & & & $7 / 22 / 96$ \\
\hline & & & 50 & $\begin{array}{l}\text { Test Container Dose Rate (mrem/hr) field for } \\
\text { format.and case restriction: }\end{array}$ & 0 & $x$ & & & $7 / 22 / 96$ \\
\hline & & & 51 & $\begin{array}{l}\text { Test Container Neutron Dose Rate field for } \\
\text { length, format, and case restriction: }\end{array}$ & 0 & $\mathrm{x}$ & & & $7 / 22 / 96$ \\
\hline & & & 52 & $\begin{array}{l}\text { Test Content Organic Volume \% field for } \\
\text { length, format, and case restriction: }\end{array}$ & 1 & & $\mathrm{x}$ & & $8 / 20 / 96$ \\
\hline & & & 53 & $\begin{array}{l}\text { Test Content Organic Weight }(\mathrm{kg}) \text { field for } \\
\text { length, format, and case restriction: }\end{array}$ & 1 & & $\mathrm{x}$ & & $8 / 14 / 96$ \\
\hline
\end{tabular}




\begin{tabular}{|c|c|c|c|c|c|c|c|c|c|}
\hline PROC \# & $\begin{array}{l}\text { PROCEDURE } \\
\text { NAME }\end{array}$ & $\underset{\#}{\text { CASE }}$ & $\begin{array}{c}\text { STE } \\
\mathbf{P} \\
\# \\
\end{array}$ & STEP NAME & $\underset{\#}{\text { REV }}$ & $\begin{array}{c}\text { PASS } \\
\text { 1st } \\
\text { TEST }\end{array}$ & $\begin{array}{l}\text { PASS } \\
\text { RETEST }\end{array}$ & COMMENTS & $\begin{array}{l}\text { SIGNOFF } \\
\text { DATE }\end{array}$ \\
\hline \multirow[t]{14}{*}{ DMS-F25 } & \multirow[t]{14}{*}{$\begin{array}{l}\text { Processed Waste Data Review \& Mod. } \\
\text { screen (DMSS1221) }\end{array}$} & \multirow[t]{14}{*}{04} & 54 & $\begin{array}{l}\text { Test Total Alpha Curies field for Autohint and } \\
\text { updatability: }\end{array}$ & 0 & $\mathrm{x}$ & & & $7 / 22 / 96$. \\
\hline & & & 55 & $\begin{array}{l}\text { Test Total Beta/Gamma Curies freld for } \\
\text { Autohint and updatability: }\end{array}$ & 0 & $\mathrm{x}$ & & & $7 / 22 / 96$ \\
\hline & & & 56 & $\begin{array}{l}\text { Test Total Dose Equivalent Curies field for } \\
\text { Autohint and updatability: }\end{array}$ & 0 & $\mathrm{x}$ & & & $7 / 22 / 96$ \\
\hline & & & 57 & $\begin{array}{l}\text { Test Total pu Equivalent Cuxies field for } \\
\text { Autohint and updatability: }\end{array}$ & 0 & $\mathrm{x}$. & & & $7 / 22 / 96$ \\
\hline & & & 58 & $\begin{array}{l}\text { Test Total PU Fissile Gram Equiv. field for } \\
\text { Autohint and updatability: }\end{array}$ & 0 & $\mathrm{x}$ & & & $7 / 22 / 96$ \\
\hline & & & 59 & Test [Return to Main 1221 Screen] button: & 0 & $\mathrm{x}$ & & & $7 / 22 / 96$ \\
\hline & & & 60 & Test [Perform Data Review] button: & 0 & $\mathrm{x}$ & & & $7 / 22 / 96$ \\
\hline & & & 61 & Test [ISOQTY] button: & 0 & $\mathrm{x}$ & & & $7 / 22 / 96$ \\
\hline & & & 62 & $\begin{array}{l}\text { Test [Return to Main } 1221 \text { Screen] button, no } \\
\text { ISOQTY records entered: }\end{array}$ & 0 & $\mathrm{x}$ & & & $7 / 22 / 96$ \\
\hline & & & 63 & Test [Perform Data Review] button: & 0 & $\mathrm{x}$ & & & $7 / 22 / 96$ \\
\hline & & & 64 & Re-test [ISOQTY] button: & 0 & $\mathrm{x}$ & & & $7 / 22 / 96$ \\
\hline & & & 65 & Test Iso Num field for required entry: & 1 & & $\mathrm{x}$ & & $8 / 14 / 96$ \\
\hline & & & 66 & Test Quantity field for required entry: & 0 & $\mathrm{x}$ & & & $7 / 22 / 96$ \\
\hline & & & 67 & Test TMU field for required entry: & 0 & $x$ & & & $7 / 22 / 96$ \\
\hline
\end{tabular}




\begin{tabular}{|c|c|c|c|c|c|c|c|c|c|}
\hline PROC \# & $\begin{array}{l}\text { PROCEDURE } \\
\text { NAME }\end{array}$ & $\underset{\#}{\text { CASE }}$ & $\begin{array}{c}\text { STE } \\
\mathbf{P} \\
\# \\
\end{array}$ & $\begin{array}{c}\text { STEP NAME } \\
\vdots\end{array}$ & $\underset{\#}{\mathrm{REV}}$ & $\begin{array}{c}\text { PASS } \\
\text { 1st } \\
\text { TEST }\end{array}$ & $\begin{array}{l}\text { PASS } \\
\text { RETEST }\end{array}$ & COMMENTS & $\begin{array}{l}\text { SIGNOFF } \\
\text { DATE }\end{array}$ \\
\hline \multirow[t]{15}{*}{ DMS-F25 } & \multirow{15}{*}{$\begin{array}{l}\text { Processed Waste Data Review \& Mod. } \\
\text { screen (DMSS1221) }\end{array}$} & \multirow[t]{15}{*}{04.} & 68 & Test Alpha Ci field for required entry: & 0 & $\mathrm{x}$ & & & $7 / 22 / 96$ \\
\hline & & & 69 & Test PE Ci field for required entry: & 0 & $\mathrm{x}$ & & & $7 / 22 / 96$ \\
\hline & & & 70 & Test Pu FGE field for required entry: & 0 & $\mathrm{x}$ & & & $7 / 22 / 96$ \\
\hline & & & 71 & Test [List of Values] button for Iso Num field: & 0 & $x$ & & & $7 / 22 / 96$ \\
\hline & & & 72 & Test Quantity field for formatting of value: & 0 & $\mathrm{x}$ & & & $7 / 22 / 96$ \\
\hline & & & 73 & $\begin{array}{l}\text { Test TMU field for length, format, case } \\
\text { restriction, and valid value: }\end{array}$ & 0 & $x$ & & & $7 / 22 / 96$ \\
\hline & & & 74 & $\begin{array}{l}\text { Test Alpha } \mathrm{Ci} \text { field for length, format, case } \\
\text { restriction, and valid value }\end{array}$ & 0 & $\mathrm{x}$ & & & $7 / 22 / 96$ \\
\hline & & & 75 & $\begin{array}{l}\text { Test PE Ci field for length, format, case } \\
\text { restriction, and valid value: }\end{array}$ & 0 & $\mathrm{x}$ & & & $7 / 22 / 96$ \\
\hline & & & 76 & $\begin{array}{l}\text { Test Pu FGE field for length, format, case } \\
\text { restriction, and valid value: }\end{array}$ & 0 & $x$ & & & $7 / 22 / 96$ \\
\hline & & & 77 & Test Isotope field for Autohint: & 0 & $\mathrm{x}$ & & & $7 / 22 / 96$ \\
\hline & & & 78 & Test Unit field for Autohint: & 1 & & $\mathrm{x}$ & & $8 / 14 / 96^{\circ}$ \\
\hline & & & 79 & $\begin{array}{l}\text { Test [Return to Main } 1221 \text { Screen] button for } \\
\text { ISOQTY screen: }\end{array}$ & 0 & $\mathrm{x}$ & & & $7 / 22 / 96$ \\
\hline & & & 80 & Test [RADDETAIL] button: & 0 & $x$ & & & $7 / 22 / 96$ \\
\hline & & & 81 & Test [PHYSCOMP] button: & 0 & $x$ & & & $7 / 22 / 96$ \\
\hline & & & 82 & $\begin{array}{l}\text { Test Description field for length, format, and } \\
\text { case restriction: }\end{array}$ & 0 & $\mathrm{x}$ & & & $7 / 22 / 96$ \\
\hline
\end{tabular}




\begin{tabular}{|c|c|c|c|c|c|c|c|c|c|}
\hline PROC \# & $\begin{array}{l}\text { PROCEDURE } \\
\text { NAME }\end{array}$ & $\underset{\#}{\text { CASE }}$ & $\begin{array}{c}\text { STE } \\
\mathrm{P} \\
\not \\
\end{array}$ & STEP NAME & $\begin{array}{c}\text { REV } \\
\#\end{array}$ & $\begin{array}{c}\text { PASS } \\
\text { 1st } \\
\text { TEST }\end{array}$ & $\begin{array}{l}\text { PASS } \\
\text { RETEST }\end{array}$ & COMMENTS & $\begin{array}{l}\text { SIGNOFF } \\
\text { DATE }\end{array}$ \\
\hline \multirow[t]{14}{*}{ DMS-F25 } & \multirow[t]{14}{*}{$\begin{array}{l}\text { Processed Waste Data Review \& Mod. } \\
\text { screen (DMSS1221) }\end{array}$} & \multirow[t]{14}{*}{04} & 83 & $\begin{array}{l}\text { Test Physical Composition Screen Volume } \\
\text { Percent field for required entry: }\end{array}$ & 1 & & $x$ & & $8 / 14 / 96$ \\
\hline & & & 84 & $\begin{array}{l}\text { Test Physical Composition Screen Weight }(\mathrm{kg}) \\
\text { field for required entry: }\end{array}$ & 0 & $\mathrm{X}$ & & & $7 / 22 / 96$ \\
\hline & & & 85 & $\begin{array}{l}\text { Test [List of Values] button for Physical } \\
\text { Composition Screen Description freld: }\end{array}$ & 0 & $X$ & & & $7 / 22 / 96$ \\
\hline & & & 86 & $\begin{array}{l}\text { Enter-values for Volume Percent and Weight } \\
(\mathrm{kg}) \text { : }\end{array}$ & 0 & $\mathrm{X}$ & & & $7 / 22 / 96$ \\
\hline & & & 87 & Test [Return to Main 1221 Screen] button: & 0 & $\mathrm{X}$ & & & $7 / 22 / 96$ \\
\hline & & & 88 & Re-test [Return to Main 1221 Screen] button: & 0 & $\mathrm{X}$ & & $\because=$ & $7 / 22 / 96$ \\
\hline & & & 89 & Test [Perform Data Review] button: & $\dot{0}$ & $\mathrm{X}$ & & & $7 / 22 / 96$ \\
\hline & & & 90 & Test [HAZDETAIL] button: & 0 & $\mathbf{x}$ & & & $7 / 22 / 96$ \\
\hline & & & 91 & Test screen for record scrolling: & 0 & $\mathrm{X}$ & & & $7 / 22 / 96$ \\
\hline & & & 92 & Test delete record function: & 0 & $X$ & & & $7 / 22 / 96$ \\
\hline & & & 93 & $\begin{array}{l}\text { Test Waste Status field for enterability, } \\
\text { format, length, and case restriction: }\end{array}$ & 0 & $\mathbf{x}$ & & - & $7 / 22 / 96$ \\
\hline & & & 94 & Test [Return to Main 1221 Screen] button: & 0 & $\mathrm{X}$ & & & $7 / 22 / 96$ \\
\hline & & & 95 & $\begin{array}{l}\text { Test Container Status field for enterability, } \\
\text { format, length, and case restriction: }\end{array}$ & 0 & $X$ & & & $7 / 22 / 96$ \\
\hline & & & 96 & $\begin{array}{l}\text { Test Waste Volume (m3) field for enterability, } \\
\text { format, length, and case restriction: }\end{array}$ & 1 & & $\mathrm{X}$ & & $8 / 14 / 96$ \\
\hline
\end{tabular}




\begin{tabular}{|c|c|c|c|c|c|c|c|c|c|}
\hline PROC \# & $\begin{array}{l}\text { PROCEDURE } \\
\text { NAME }\end{array}$ & $\begin{array}{c}\text { CASE } \\
\#\end{array}$ & $\begin{array}{l}\text { STE } \\
\mathbf{P} \\
\#\end{array}$ & STEP NAME & $\underset{\#}{\text { REV }}$ & $\begin{array}{c}\text { PASS } \\
\text { lst } \\
\text { TEST }\end{array}$ & $\begin{array}{c}\text { PASS } \\
\text { RETEST }\end{array}$ & COMMENTS & $\begin{array}{l}\text { SIGNOFF } \\
\text { DATE }\end{array}$ \\
\hline \multirow[t]{11}{*}{ DMS-F25 } & \multirow[t]{11}{*}{$\begin{array}{l}\text { Processed Waste Data Review \& Mod. } \\
\text { screen (DMS\$1221) }\end{array}$} & \multirow[t]{11}{*}{04} & 97 & $\begin{array}{l}\text { Test Haz. Property Code field for enterability, } \\
\text { format, length, and case restriction: }\end{array}$ & 1 & & $\mathrm{X}$ & & $8 / 14 / 96$ \\
\hline & & & 98 & $\begin{array}{l}\text { Test Flashpoint field for enterability, format, } \\
\text { length, case restriction, and valid value: }\end{array}$ & 0 & $x$ & & & $7 / 22 / 96$ \\
\hline & & & 99 & Test pH field for enterability: & 1 & & $\mathrm{X}$ & & $8 / 14 / 96$ \\
\hline & & & 100 & Test Designation Code for Autohint: & 1 & & $\mathrm{x}$ & & $8 / 14 / 96$ \\
\hline & & & 101 & $\begin{array}{l}\text { Test Item Type field for enterability, format, } \\
\text { length, and case restriction: }\end{array}$ & 0 & $\mathrm{x}$ & & & $7 / 22 / 96$ \\
\hline & & & 102 & $\begin{array}{l}\text { Test Subtype field for enterability, format, } \\
\text { length, and case restriction: }\end{array}$ & 0 & $\mathrm{X}$ & & & $7 / 22 / 96$ \\
\hline & & & 103 & $\begin{array}{l}\text { Test Contents/Description field for enterability, } \\
\text { format, length, and case restriction: }\end{array}$ & 0 & $\mathrm{X}$ & & & $7 / 22 / 96$ \\
\hline & & & 104 & $\begin{array}{l}\text { Test Removed from Service field for } \\
\text { enterability, format, length, and case } \\
\text { restriction: }\end{array}$ & 0 & $x$ & & . & $7 / 22 / 96$ \\
\hline & & & 105 & $\begin{array}{l}\text { Test Concentration (ppM) field for enterability, } \\
\text { format, length, and case restriction: }\end{array}$ & 0 & $\mathrm{X}$ & & & $7 / 22 / 96$ \\
\hline & & & 106 & $\begin{array}{l}\text { Test PCB Waste Weight }(\mathrm{kg}) \text { field for } \\
\text { enterability, format, length, and case } \\
\text { restriction: }\end{array}$ & 0 & $X$ & & & $7 / 22 / 96$ \\
\hline & & & 107 & $\begin{array}{l}\text { Test Other Landban } 1 \text { field for enterability, } \\
\text { format, length, and case restriction: }\end{array}$ & 0 & $\mathrm{X}$ & & & $7 / 22 / 96$ \\
\hline
\end{tabular}




\begin{tabular}{|c|c|c|c|c|c|c|c|c|c|}
\hline PROC \# & $\begin{array}{l}\text { PROCEDURE } \\
\text { NAME }\end{array}$ & $\underset{\#}{\mathrm{CASE}}$ & $\begin{array}{c}\text { STE } \\
\mathbf{P} \\
\#\end{array}$ & STEP NAME & $\underset{\#}{\text { REV }}$ & $\begin{array}{l}\text { PASS } \\
\text { 1st } \\
\text { TEST }\end{array}$ & $\begin{array}{c}\text { PASS } \\
\text { RETEST }\end{array}$ & COMMENTS & $\begin{array}{l}\text { SIGNOFF } \\
\text { DATE }\end{array}$ \\
\hline \multirow[t]{15}{*}{ DMS-F25 } & \multirow[t]{15}{*}{$\begin{array}{l}\text { Processed Waste Data Review \& Mod. } \\
\text { screen (DMSS1221) }\end{array}$} & \multirow[t]{15}{*}{04} & 108 & $\begin{array}{l}\text { Test Other Landban } 2 \text { field for enterability, } \\
\text { format, length, and case restriction: }\end{array}$ & 0 & $\mathrm{x}$ & & & $7 / 22 / 96$ \\
\hline & & & 109 & $\begin{array}{l}\text { Test Other Landban } 3 \text { freld for enterability, } \\
\text { format, length, and case restriction: }\end{array}$ & 0 & $\mathrm{x}$ & & & $7 / 22 / 96$ \\
\hline & & & 110 & Test tabbing sequence: & 0 & $\mathrm{x}$ & & & $7 / 22 / 96$ \\
\hline & & & 111 & $\begin{array}{l}\text { Test Dangerous Waste Numbers field for } \\
\text { Autohint: }\end{array}$ & 0 & $\mathrm{x}$ & & & $7 / 22 / 96$ \\
\hline & & & 112 & Test Landban field for Autohint: & 0 & $x$ & & & $7 / 22 / 96$ \\
\hline & & & 113 & Test [DW_NUM] button: & 0 & $\mathrm{x}$ & & & $7 / 22 / 96$ \\
\hline & & & 114 & Test DW Number freld for required entry: & 1 & & $\mathrm{X}$ & & $8 / 14 / 96$ \\
\hline & & & 115 & $\begin{array}{l}\text { Test Landban field for editability, length, } \\
\text { format, case restriction, and valid value: }\end{array}$ & 0 & $\mathrm{x}$ & & & $7 / 22 / 96$ \\
\hline & & & 116 & Test Description field for Autohint: & 1 & & $\mathrm{x}$ & & $8 / 14 / 96$ \\
\hline & & & 117 & Test Sort Order field for Autohint: & 0 & $\mathrm{x}$ & & & $7 / 22 / 96$ \\
\hline & & & 118 & Test [Return to HAZDETAIL Screen] button: & 0 & $\mathrm{x}$ & & & $7 / 22 / 96$. \\
\hline & & & 119 & Test [Return to Main 1221 ScreenI button: & 0 & $\mathrm{x}$ & & & $7 / 22 / 96$ \\
\hline & & & 120 & Test [Perform Data Review] button: & 0 & $\mathrm{x}$ & & & $7 / 22 / 96$ \\
\hline & & & 121 & Test [CHEMCOMP] button: & 0 & $\mathrm{x}$ & & & $7 / 22 / 96$ \\
\hline & & & 122 & Test ID field for required entry: & 0 & $\mathrm{x}$ & & & $7 / 22 / 96$ \\
\hline
\end{tabular}




\begin{tabular}{|c|c|c|c|c|c|c|c|c|c|}
\hline PROC \# & $\begin{array}{l}\text { PROCEDURE } \\
\text { NAME }\end{array}$ & $\underset{\#}{\text { CASE }}$ & $\begin{array}{c}\text { STE } \\
\mathbf{P} \\
\# \\
\end{array}$ & STEP NAME & $\underset{\#}{\mathrm{REV}}$ & $\begin{array}{c}\text { PASS } \\
\text { 1st } \\
\text { TEST }\end{array}$ & $\begin{array}{c}\text { PASS } \\
\text { RETEST }\end{array}$ & COMMENTS & $\begin{array}{l}\text { SIGNOFF } \\
\text { DATE }\end{array}$ \\
\hline \multirow[t]{14}{*}{ DMS-F25 } & \multirow[t]{14}{*}{$\begin{array}{l}\text { Processed Waste Data Review \& Mod. } \\
\text { screen (DMSS1221) }\end{array}$} & \multirow[t]{14}{*}{04} & 123 & $\begin{array}{l}\text { Test ID field for format, length, and case } \\
\text { restriction: }\end{array}$ & 0 & $\mathrm{x}$ & & & $7 / 22 / 96$ \\
\hline & & & 124 & $\begin{array}{l}\text { Test [List of Values] button from Chemical } \\
\text { Component Screen: }\end{array}$ & 0 & $\mathrm{x}$ & & & $7 / 22 / 96$ \\
\hline & & & 125 & Test tabbing sequence: & 0 & $\mathrm{x}$ & & & $7 / 22 / 96$ \\
\hline & & & 126 & $\begin{array}{l}\text { Test Weight (kg) field for case restriction, } \\
\text { format, and length: }\end{array}$ & 0 & $\mathrm{x}$ & & & $7 / 22 / 96$ \\
\hline & & & 127 & $\begin{array}{l}\text { Test ID field for direct entry (not using List of } \\
\text { Values): }\end{array}$ & 0 & $\mathrm{x}$ & & & $7 / 22 / 96$ \\
\hline & & & 128 & $\begin{array}{l}\text { Test Weight Percent field for case restriction, } \\
\text { format, length, and valid value: }\end{array}$ & 1 & & $\mathrm{x}$ & & $8 / 14 / 96$ \\
\hline & & & 129 & Test Description field for editability: & 0 & $\mathrm{x}$ & & & $7 / 22 / 96$ \\
\hline & & & 130 & Test [Return to Main 1221 Screen] button: & 0 & $\mathrm{x}$ & & & $7 / 22 / 96$ \\
\hline & & & 131 & Test [Perform Data Review] button: & 0 & $\mathrm{x}$ & & & $7 / 22 / 96$ \\
\hline & & & 132 & Test [APPMSDS] button & 0 & $\mathrm{x}$ & & & $7 / 22 / 96$ \\
\hline & & & 133 & Test MSDS ID field for required entry: & 0 & $\mathrm{x}$ & & & $7 / 22 / 96$ \\
\hline & & & 134 & $\begin{array}{l}\text { Test MSDS ID field for enterability, format, } \\
\text { length, case restriction, and valid value: }\end{array}$ & 0 & $\mathrm{x}$ & & & $7 / 22 / 96$ \\
\hline & & & 135 & Test MSDS Description field for Autohint: & 0 & $\mathrm{x}$ & & & $7 / 22 / 96$ \\
\hline & & & 136 & $\begin{array}{l}\text { Test [Return to Main } 1221 \text { Screen] button on } \\
\text { Applicable MSDS Screen: }\end{array}$ & 0 & $\mathrm{x}$ & & & $7 / 22 / 96$ \\
\hline
\end{tabular}




\begin{tabular}{|c|c|c|c|c|c|c|c|c|c|}
\hline PROC \# & $\begin{array}{l}\text { PROCEDURE } \\
\text { NAME }\end{array}$ & $\underset{\#}{\text { CASE }}$ & $\begin{array}{c}\text { STE } \\
\mathbf{P} \\
\#\end{array}$ & STEP NAME & $\underset{\#}{\mathrm{REV}}$ & $\begin{array}{l}\text { PASS } \\
\text { 1st } \\
\text { TEST }\end{array}$ & $\begin{array}{c}\text { PASS } \\
\text { RETEST }\end{array}$ & COMMENTS & $\begin{array}{l}\text { SIGNOFF } \\
\text { DATE }\end{array}$ \\
\hline \multirow[t]{11}{*}{ DMS-F25 } & \multirow{11}{*}{$\begin{array}{l}\text { Processed Waste Data Review \& Mod. } \\
\text { screen (DMSS1221) }\end{array}$} & \multirow[t]{11}{*}{04} & 137 & Test [Perform Data Review] button: & 0 & $\mathrm{x}$ & & & $7 / 22 / 96$ \\
\hline & & & 138 & Test [CONREL] button: & 0 & $x$ & & & $7 / 22 / 96$ \\
\hline & & & 139 & $\begin{array}{l}\text { Test Container Relationship Display Tree for } \\
\text { enterability: }\end{array}$ & 0 & $\mathrm{x}$ & & & $7 / 22 / 96$ \\
\hline & & & 140 & $\begin{array}{l}\text { Enter a query in Container Relationship } \\
\text { Display Tree block: }\end{array}$ & 0 & $\mathrm{x}$ & & & $7 / 22 / 96$ \\
\hline & & & 141 & $\begin{array}{l}\text { Test Next Block function key from Container } \\
\text { Relationship Display Tree block: }\end{array}$ & 0 & $x$ & & & $7 / 22 / 96$. \\
\hline & & & 142 & $\begin{array}{l}\text { Test CONREL Record Maintenance From PIN } \\
\text { for format, length, case restriction, and valid } \\
\text { entry: }\end{array}$ & 0 & $x$ & & & $7 / 22 / 96$ \\
\hline & & & 143 & $\begin{array}{l}\text { Test CONREL Record Maintenance To PIN } \\
\text { for format, length, case restriction, and valid } \\
\text { entry: }\end{array}$ & 0 & $\mathrm{x}$ & & & $7 / 22 / 96$ \\
\hline & & & 144 & $\begin{array}{l}\text { Test CONREL Record Maintenance Date for } \\
\text { format, length, case restriction, and valid } \\
\text { entry: }\end{array}$ & 0 & $\mathrm{x}$ & & & $7 / 22 / 96$ \\
\hline & & & 145 & $\begin{array}{l}\text { Test CONREL Record Maintenance Cd for } \\
\text { format, length, case restriction, and valid } \\
\text { entry: }\end{array}$ & 0 & $x$ & & & $7 / 22 / 96$ \\
\hline & & & 146 & $\begin{array}{l}\text { Enter a query in CONREL Record } \\
\text { Maintenance block: }\end{array}$ & 1 & & $\mathrm{x}$ & & $8 / 14 / 96$ \\
\hline & & & 147 & $\begin{array}{l}\text { Test up and down function keys in CONREL } \\
\text { Record Maintenance block; }\end{array}$ & 0 & $\mathrm{x}$ & & & $7 / 22 / 96$ \\
\hline
\end{tabular}




\begin{tabular}{|c|c|c|c|c|c|c|c|c|c|}
\hline PROC \# & $\begin{array}{l}\text { PROCEDURE } \\
\text { NAME }\end{array}$ & $\underset{\#}{\text { CASE }}$ & $\begin{array}{c}\text { STE } \\
\mathbf{P} \\
\# \\
\end{array}$ & STEP NAME & $\underset{\#}{\mathrm{REV}}$ & $\begin{array}{l}\text { PASS } \\
\text { 1st } \\
\text { TEST }\end{array}$ & $\begin{array}{l}\text { PASS } \\
\text { RETEST }\end{array}$ & COMMENTS & $\begin{array}{l}\text { SIGNOFF } \\
\text { DATE }\end{array}$ \\
\hline \multirow[t]{15}{*}{ DMS-F25 } & \multirow[t]{15}{*}{$\begin{array}{l}\text { Processed Waste Data Review \& Mod. } \\
\text { screen (DMSS1221) }\end{array}$} & \multirow[t]{15}{*}{04} & 148 & $\begin{array}{l}\text { Test delete record function in CONREL } \\
\text { Record Maintenance block: }\end{array}$ & 0 & $\mathrm{x}$ & & & $7 / 22 / 96$ \\
\hline & & & 149 & Test From PIN for updatability: & 0 & $\mathrm{x}$ & & & $7 / 22 / 96$ \\
\hline & & & 150 & Test To PIN for updatability: & 0 & $\mathrm{x}$ & & & $7 / 22 / 96$ \\
\hline & & & 151 & Test Date for updatability: & 0 & $\mathrm{x}$ & & & $7 / 22 / 96$ \\
\hline & & & 152 & Test Cd for updatability: & 0 & $\mathrm{x}$ & & & $7 / 22 / 96$ \\
\hline & & & 153 & Test [Update CONREL Records] button: & 0 & $x$ & & & $7 / 22 / 96$ \\
\hline & & & 154 & $\begin{array}{l}\text { Test [Insert] button for CONREL Record } \\
\text { Maintenance block: }\end{array}$ & 0 & $x$ & $\dot{0}$ & & $7 / 22 / 96$ \\
\hline & & & 155 & $\begin{array}{l}\text { Test duplicate record function in CONREL } \\
\text { Record Maintenance block: }\end{array}$ & 0 & $x$ & . & & $7 / 22 / 96$ \\
\hline & & & 156 & Test [Commit] button: & 0 & $\mathrm{x}$ & & & $7 / 22 / 96$ \\
\hline & & & 157 & $\begin{array}{l}\text { Create CONREL records for PIN being } \\
\text { reviewed: }\end{array}$ & 0 & $\mathrm{x}$ & & . & $7 / 22 / 96$ \\
\hline & & & 158 & $\begin{array}{l}\text { Test displayed CONREL relationship for } \\
\text { editability: }\end{array}$ & 0 & $\mathrm{x}$ & & & $7 / 22 / 96$ \\
\hline & & & 159 & Test [Return to Main 1221 Screen] button: & 0 & $\mathrm{x}$ & & & $7 / 22 / 96$ \\
\hline & & & 160 & Test [Perform Data Review] button: & 0 & $\mathrm{x}$ & & & $7 / 22 / 96$ \\
\hline & & & 161 & Retest [CONREL] button: & 0 & $\mathrm{x}$ & & & $7 / 22 / 96$ \\
\hline & & & 162 & Test [Update CONREL Records] button: & 0 & $x$ & & & $7 / 22 / 96$ \\
\hline
\end{tabular}




\begin{tabular}{|c|c|c|c|c|c|c|c|c|c|}
\hline PROC \# & $\begin{array}{l}\text { PROCEDURE } \\
\text { NAME }\end{array}$ & $\underset{\#}{\text { CASE }}$ & $\begin{array}{c}\text { STE } \\
\mathrm{P} \\
\# \\
\end{array}$ & STEP NAME & $\underset{\#}{\mathrm{REV}}$ & $\begin{array}{c}\text { PASS } \\
\text { 1st } \\
\text { TEST }\end{array}$ & $\begin{array}{c}\text { PASS } \\
\text { RETEST }\end{array}$ & COMMENTS & $\begin{array}{l}\text { SIGNOFF } \\
\text { DATE }\end{array}$ \\
\hline \multirow[t]{15}{*}{ DMS-F25 } & \multirow{15}{*}{$\begin{array}{l}\text { Processed Waste Data Review \& Mod. } \\
\text { screen (DMSS1221) }\end{array}$} & \multirow[t]{4}{*}{04} & 163 & Test [Return to Main 1221 Screen] button: & 0 & $\mathrm{x}$ & & & $7 / 22 / 96$ \\
\hline & & & 164 & Test [Perform Data Review] button: & 0 & $\mathrm{x}$ & & & $7 / 22 / 96$ \\
\hline & & & 166 & Test [Exit] button: & 0 & $\mathrm{x}$ & & & $7 / 22 / 96$ \\
\hline & & & 167 & Exit the DMS system. & 0 & $\mathrm{x}$ & & & $7 / 22 / 96$ \\
\hline & & \multirow[t]{11}{*}{05} & 1 & Run pre-test query ' $\mathrm{f} 25.05 \mathrm{q} \mathrm{b}^{\prime}$. & 0 & $\mathrm{x}$ & & & $7 / 22 / 96$ \\
\hline & & & 2 & $\begin{array}{l}\text { Logon to DMS system as a user (USER1) with } \\
\text { test .role UPDATE privileges. }\end{array}$ & 0 & $\mathrm{x}$ & & & $7 / 22 / 96$ \\
\hline & & & 3 & $\begin{array}{l}\text { Select the Processed Waste Data Review \& } \\
\text { Mod. screen (DMSS1221) from the DMS Main } \\
\text { Menu: }\end{array}$ & 0 & $\mathrm{X}$ & & & $7 / 22 / 96$ \\
\hline & & & 4 & Test [Perform Data Review] button: & 0 & $\mathrm{x}$ & & & $7 / 22 / 96$ \\
\hline & & & 5 & Test [List of Values] button for drum PIN: & 0 & $\mathrm{X}$ & & & $7 / 22 / 96$ \\
\hline & & & 6 & Test [WASTE] button: & 0 & $\mathrm{x}$ & & & $7 / 22 / 96$ \\
\hline & & & 7 & $\begin{array}{l}\text { Test Primary Waste Type field for } \\
\text { updatability: }\end{array}$ & 0 & $\mathrm{x}$ & & & $7 / 22 / 96$ \\
\hline & & & 8 & Test [Return to Main 1221 Screen] button: & 0 & $\mathrm{x}$ & & & $7 / 22 / 96$ \\
\hline & & & 9 & Test [RADDETAIL] button: & 0 & $\mathrm{x}$ & & & $7 / 22 / 96$ \\
\hline & & & 10 & Test [List of Values] for Waste Category: & 0 & $\mathrm{x}$ & &. & $7 / 22 / 96$ \\
\hline & & & 11 & Test [Return to Main 1221 Screen] button: & 0 & $\mathrm{x}$ & & & $7 / 22 / 96$ \\
\hline
\end{tabular}




\begin{tabular}{|c|c|c|c|c|c|c|c|c|c|}
\hline PROC \# & $\begin{array}{l}\text { PROCEDURE } \\
\text { NAME }\end{array}$ & $\underset{\#}{\mathrm{CASE}}$ & $\begin{array}{l}\text { STE } \\
\mathbf{P} \\
\#\end{array}$ & STEP NAME & $\underset{\#}{\text { REV }}$ & $\begin{array}{l}\text { PASS } \\
\text { 1st } \\
\text { TEST }\end{array}$ & $\begin{array}{c}\text { PASS } \\
\text { RETEST }\end{array}$ & COMMENTS & $\begin{array}{l}\text { SIGNOFF } \\
\text { DATE }\end{array}$ \\
\hline \multirow[t]{16}{*}{ DMS-F25 } & \multirow{16}{*}{$\begin{array}{l}\text { Processed Waste Data Review \& Mod. } \\
\text { screen (DMSS1221) }\end{array}$} & \multirow[t]{13}{*}{05} & 12 & Test [ISOQTY] button: & 0 & $\mathrm{x}$ & & . & $7 / 22 / 96$ \\
\hline & & & 13 & $\begin{array}{l}\text { Test [Return to Main } 1221 \text { Screen] button for } \\
\text { ISOQTY screen: }\end{array}$ & 0 & $\mathrm{x}$ & & & $7 / 22 / 96$ \\
\hline & & & 14 & Test [PHYSCOMP] button: & 0 & $x$ & & & $7 / 22 / 96$. \\
\hline & & & 15 & Test [Return to Main 1221 Screen] button: & 0 & $\mathrm{x}$ & & & $7 / 22 / 96$ \\
\hline & & & 16 & Test [HAZDETAlL] button: & 0 & $\mathrm{x}$ & & & $7 / 22 / 96$ \\
\hline & & & 17 & Test delete record function: & 0 & $\mathrm{x}$ & & & $7 / 22 / 96$ \\
\hline & & & 18 & Test [Return to Main 1221 Screen] button: & 0 & $\mathrm{x}$ & & & $7 / 22 / 96$ \\
\hline & & & 19 & Re-test [HAZDETAIL] button: & 0 & $\mathrm{x}$ & & & $7 / 22 / 96$ \\
\hline & & & 20 & Test [CHEMCOMP] button: & 0 & $\mathrm{x}$ & & & $7 / 22 / 96$ \\
\hline & & & 21 & Test [Perform Data Review] button: & 1 & & $\mathbf{x}$ & & $8 / 20 / 96$ \\
\hline & & & 22 & Test [Exit] button: & 0 & $\mathrm{x}$ & & & $7 / 22 / 96$ \\
\hline & & & 23 & Exit the DMS system. & 0 & $\mathrm{x}$ & & & $7 / 22 / 96$ \\
\hline & & & 24 & Perform post-test query ' $\$ 25.05 q \mathrm{a}$ '. & 0 & $\mathrm{x}$ & & . & $7 / 22 / 96$ \\
\hline & & \multirow[t]{3}{*}{06} & 1 & Run pre-test query ' $\mathrm{f} 25.06 \mathrm{q}$ b'. & 0 & $\mathrm{x}$ & & & $7 / 22 / 96$ \\
\hline & & & 2 & $\begin{array}{l}\text { Logon to DMS system as a user (USER1) with } \\
\text { test role UPDATE privileges. }\end{array}$ & 0 & $\mathrm{x}$ & & & $7 / 22 / 96$ \\
\hline & & & 3 & $\begin{array}{l}\text { Select the Processed Waste Data Review \& } \\
\text { Mod. screen (DMSS1221) from the DMS Main } \\
\text { Menu: }\end{array}$ & 0 & $x$ & & & $7 / 22 / 96$ \\
\hline
\end{tabular}




\begin{tabular}{|c|c|c|c|c|c|c|c|c|c|}
\hline PROC \# & $\begin{array}{l}\text { PROCEDURE } \\
\text { NAME }\end{array}$ & $\underset{\#}{\text { CASE }}$ & $\begin{array}{c}\text { STE } \\
P \\
\# \\
\#\end{array}$ & STEP NAME & $\underset{\#}{\text { REV }}$ & $\begin{array}{c}\text { PASS } \\
\text { 1st } \\
\text { TEST }\end{array}$ & $\begin{array}{l}\text { PASS } \\
\text { RETEST }\end{array}$ & COMOMENTS & $\begin{array}{l}\text { SIGNOFF } \\
\text { DATE }\end{array}$ \\
\hline \multirow{16}{*}{ DMS-F25 } & \multirow{16}{*}{$\begin{array}{l}\text { Processed Waste Data Review \& Mod. } \\
\text { screen (DMSS1221) }\end{array}$} & \multirow[t]{16}{*}{06} & 4 & Test Drum Selection radio button: & 0 & $\mathrm{x}$ & & & $7 / 22 / 96$ \\
\hline & & & 5 & Test [List of Values] button for drum PIN: & 0 & $\mathrm{x}$ & & & $7 / 22 / 96$ \\
\hline & & & 6 & Test [Perform Data Review] button: & 1 & & $\mathrm{x}$ & & $8 / 20 / 96$ \\
\hline & & & 7 & Test [WASTE] button: & 0 & $\mathrm{x}$ & & & $7 / 22 / 96$ \\
\hline & & & 8 & Test [Return to Main 1221 Screen] button: & 0 & $\mathrm{x}$ & & & $7 / 22 / 96$ \\
\hline & & & 9 & Test [RADDETAIL] button: & 0 & $\mathrm{x}$ & & & $7 / 22 / 96$ \\
\hline & & & 10 & Test Waste Category field for editability: & 0 & $\mathrm{x}$ & & & $7 / 22 / 96$ \\
\hline & & & 11 & $\begin{array}{l}\text { Enter valid value for Content Organic Volume } \\
\% \text { field: }\end{array}$ & 0 & $\mathrm{x}$ & & & $7 / 22 / 96$ \\
\hline & & & 12 & $\begin{array}{l}\text { Test Content Organic Weight }(\mathrm{kg}) \text { field for } \\
\text { format: }\end{array}$ & 0 & $\mathrm{x}$ & & & $7 / 22 / 96$ \\
\hline & & & 13 & Test [Return to Main 1221 Screen] button: & 0 & $\mathrm{x}$ & & & $7 / 22 / 96$ \\
\hline & & & 14 & Re-test [RADDETAIL] button: & 0 & $\mathrm{x}$ & & & $7 / 22 / 96$ \\
\hline & & & 15 & Test [HAZDETAIL] button: & 0 & $\mathrm{X}$ & & & $7 / 22 / 96$ \\
\hline & & & 16 & Test [CHEMCOMP] button: & 0 & $\mathrm{x}$ & & & $7 / 22 / 96$ \\
\hline & & & 17 & Test [CONREL] button: & 0 & $\mathrm{x}$ & & & $7 / 22 / 96$ \\
\hline & & & 18 & Test [Update CONREL Records] button: & 0 & $\mathrm{x}$ & & & $7 / 22 / 96$ \\
\hline & & & 19 & $\begin{array}{l}\text { Test Next Block function key from Container } \\
\text { Relationship Display Tree block: }\end{array}$ & 0 & $\mathrm{x}$ & & & $7 / 22 / 96$ \\
\hline
\end{tabular}


DMS PHASE I TEST REPORT

APPENDIX B: FUNCTIONAL TEST RESULTS
DOC NUMBER: HNF-SD-WM-TRP-287

REVISION: 0

DATE: $9 / 27 / 96$

\begin{tabular}{|c|c|c|c|c|c|c|c|c|c|}
\hline PROC \# & $\begin{array}{l}\text { PROCEDURE } \\
\text { NAME }\end{array}$ & $\begin{array}{c}\text { CASE } \\
\#\end{array}$ & $\begin{array}{c}\text { STE } \\
\mathbf{P} \\
\#\end{array}$ & STEP NAME & $\begin{array}{c}\text { REV } \\
\#\end{array}$ & $\begin{array}{c}\text { PASS } \\
\text { Ist } \\
\text { TEST }\end{array}$ & $\begin{array}{l}\text { PASS } \\
\text { RETEST }\end{array}$ & COMMENTS & $\begin{array}{l}\text { SIGNOFF } \\
\text { DATE }\end{array}$ \\
\hline \multirow[t]{16}{*}{ DMS-F25 } & \multirow{16}{*}{$\begin{array}{l}\text { Processed Waste Data Review \& Mod. } \\
\text { screen (DMSS1221) }\end{array}$} & \multirow[t]{16}{*}{06} & 20 & Enter value for From PIN: & 0 & $\mathrm{X}$ & & & $7 / 22 / 96$ \\
\hline & & & 21 & Enter value for To PIN: & 0 & $\mathrm{X}$ & & & $7 / 22 / 96$ \\
\hline & & & 22 & Enter value for Date: & 0 & $\dot{x}$ & & & $7 / 22 / 96$ \\
\hline & & & 23 & Test [List of Values] button for Cd field: & 0 & $x$ & & & $7 / 22 / 96$ \\
\hline & & & 24 & Test [Update CONREL Records] button: & 1 & & $x$ & & $8 / 20 / 96$ \\
\hline & & & 25 & Enter another container relationship record: & 0 & $\mathrm{X}$ & & & $7 / 22 / 96$ \\
\hline & & & 26 & Test [Commit] button: & 0 & $\mathrm{X}$ & & & $7 / 22 / 96$ \\
\hline & & & 27 & Test [Update CỌNREL Records] button: & 0 & $x$ & & & $7 / 22 / 96$ \\
\hline & & & 28 & Test [Return to Main 1221 Screen] button: & 0 & $\mathrm{X}$ & & & $7 / 22 / 96$ \\
\hline & & & 29 & Re-test [CONREL] button: & 0 & $x$ & & & $7 / 22 / 96$ \\
\hline & & & 30 & Test [Data Review Complete] button: & 0 & $\mathrm{X}$ & & & $7 / 22 / 96$ \\
\hline & & & 31 & Test [Exit] button: & 0 & $X$ & & & $7 / 22 / 96$ \\
\hline & & & 32. & Exit the DMS system. & 0 & $\mathrm{x}$ & & & $7 / 22 / 96$ \\
\hline & & & 33 & Perform post-test query ' $25.06 \mathrm{q} \mathrm{a}^{\prime}$. & 0 & $x$ & 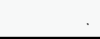 & & $7 / 22 / 96$ \\
\hline & & & 1 & Run pre-test query ' $25.07 \mathrm{q} b$ '. & 0 & $\mathrm{x}$ & & . & $7 / 22 / 96$ \\
\hline & & & 2 & $\begin{array}{l}\text { Logon to DMS system as a user (USER1) with } \\
\text { test role UPDATE privileges. }\end{array}$ & 0 & $\mathrm{x}$ & & & $7 / 22 / 96$ \\
\hline
\end{tabular}


DATE: $9 / 27 / 96$

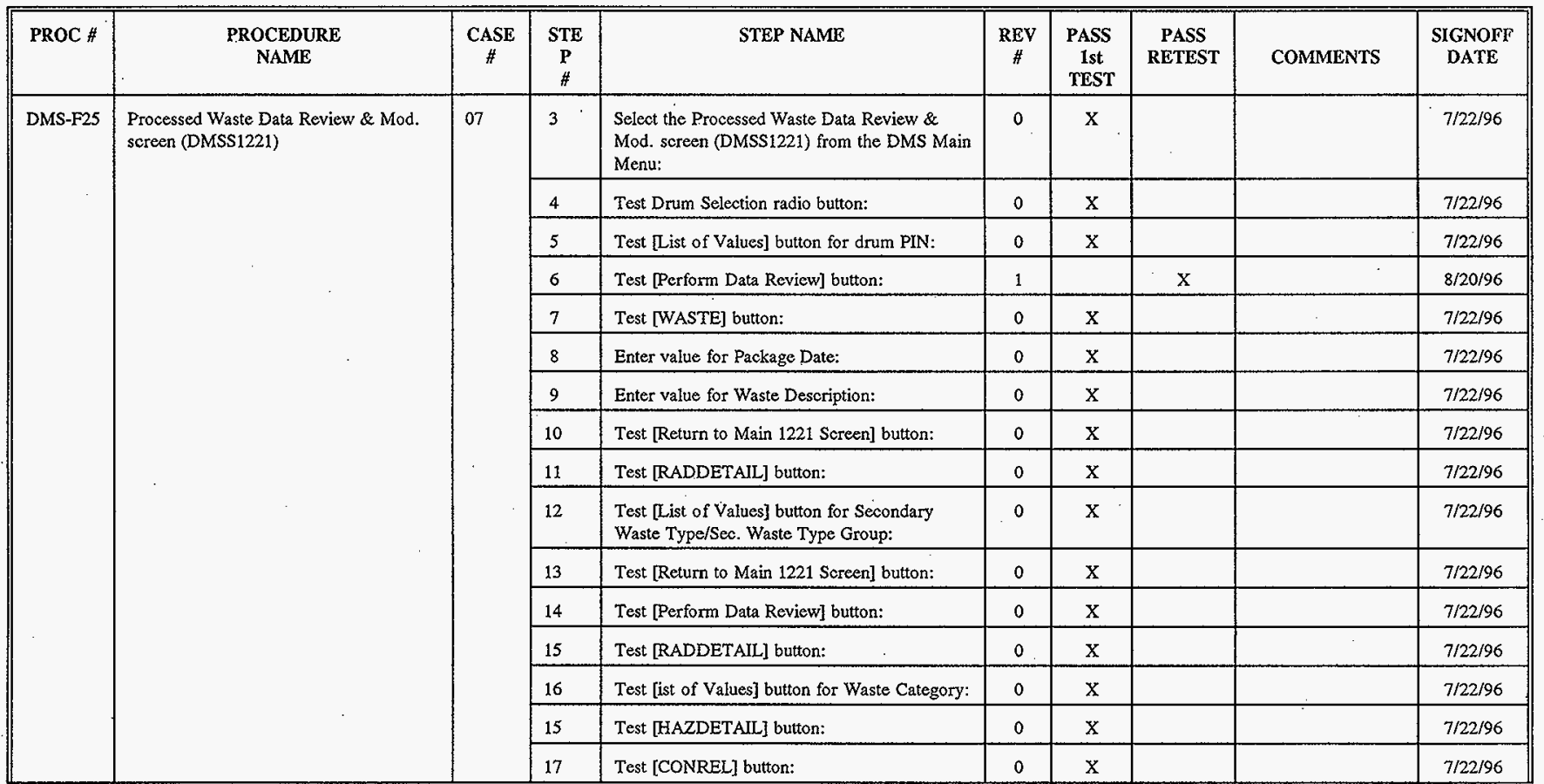




\begin{tabular}{|c|c|c|c|c|c|c|c|c|c|}
\hline PROC \# & $\begin{array}{l}\text { PROCEDURE } \\
\text { NAME }\end{array}$ & $\underset{\#}{\text { CASE }}$ & $\begin{array}{c}\text { STE } \\
\mathbf{P} \\
\#\end{array}$ & STEP NAME & $\underset{\#}{\mathrm{REV}}$ & $\begin{array}{c}\text { PASS } \\
\text { 1st } \\
\text { TEST }\end{array}$ & $\begin{array}{c}\text { PASS } \\
\text { RETEST }\end{array}$ & COMMENTS & $\begin{array}{l}\text { SIGNOFF } \\
\text { DATE }\end{array}$ \\
\hline \multirow[t]{15}{*}{ DMS-F25 } & \multirow[t]{15}{*}{$\begin{array}{l}\text { Processed Waste Data Review \& Mod. } \\
\text { screen (DMSS1221) }\end{array}$} & \multirow[t]{15}{*}{07} & 18 & $\begin{array}{l}\text { Test Next Block function key from Container } \\
\text { Relationship Display Tree block: }\end{array}$ & 0 & $\mathrm{x}$ & & & $7 / 22 / 96$ \\
\hline & & & 19 & Enter value for From PIN: & 0 & $\mathrm{x}$ & & & $7 / 22 / 96$ \\
\hline & & & 20 & Enter value for To PIN: & 0 & $\mathrm{x}$ & & & $7 / 22 / 96$ \\
\hline & & & 21 & Enter value for Date: & 0 & $\mathrm{x}$ & & & $7 / 22 / 96$ \\
\hline & & & 22 & Test [List of Values] button for $\mathrm{Cd}$ field: & 0 & $\mathrm{x}$ & & & $7 / 22 / 96$ \\
\hline & & & 23 & Test [Update CONREL Records] button: & 0 & $\mathrm{x}$ & & & $7 / 22 / 96$ \\
\hline & & & 25 & Test [Commit] button: & 0 & $\mathrm{x}$ & & & $7 / 22 / 96$ \\
\hline & & & 27 & Test [Return to Main 1221 Screen] button: & 0 & $\mathrm{x}$ & & & $7 / 22 / 96$ \\
\hline & & & 28 & Re-test [CONREL] button: & 0 & $\mathrm{x}$ & & & $7 / 22 / 96$ \\
\hline & & & 29 & Test [Perform Data Review] button: & 0 & $\mathrm{x}$ & & & $7 / 22 / 96$ \\
\hline & & & 31 & Test [Exit] button: & 0 & $\mathrm{x}$ & & & $7 / 22 / 96$ \\
\hline & & & 32 & Exit the DMS system. & 0 & $\mathrm{x}$ & & & $7 / 22 / 96$ \\
\hline & & & 33 & Perform post-test query ' $25.07 \mathrm{q}^{\mathrm{a}}$ '. & 0 & $\mathrm{x}$ & & & $7 / 22 / 96$ \\
\hline & & & 1 & Run pre-test query ' $\mathrm{f} 25.08 \mathrm{q}$ b'. & 0 & $\mathrm{x}$ & & & $7 / 22 / 96$ \\
\hline & & & 2 & $\begin{array}{l}\text { Logon to DMS system as a user (USER1) with } \\
\text { test role UPDATE privileges. }\end{array}$ & 0 & $\mathrm{x}$ & & & $7 / 22 / 96$ \\
\hline
\end{tabular}




\begin{tabular}{|c|c|c|c|c|c|c|c|c|c|}
\hline PROC \# & $\begin{array}{l}\text { PROCEDURE } \\
\text { NAME }\end{array}$ & $\underset{\#}{\mathrm{CASE}}$ & $\begin{array}{c}\text { STE } \\
\mathbf{P} \\
\#\end{array}$ & STEP NAME & $\begin{array}{c}\text { REV } \\
\#\end{array}$ & $\begin{array}{l}\text { PASS } \\
\text { 1st } \\
\text { TEST }\end{array}$ & $\begin{array}{c}\text { PASS } \\
\text { RETEST }\end{array}$ & COMMENTS & $\begin{array}{l}\text { SIGNOFF } \\
\text { DATE }\end{array}$ \\
\hline \multirow[t]{13}{*}{ DMS-F25 } & \multirow[t]{13}{*}{$\begin{array}{l}\text { Processed Waste Data Review \& Mod. } \\
\text { screen (DMSS1221) }\end{array}$} & \multirow[t]{13}{*}{08} & 3 & $\begin{array}{l}\text { Select the Processed Waste Data Review \& } \\
\text { Mod. screen (DMSS1221) from the DMS Main } \\
\text { Menu: }\end{array}$ & 0 & $\mathrm{x}$ & & & $7 / 22 / 96$ \\
\hline & & & 4 & $\begin{array}{l}\text { Enter value for drum PIN (LLW, not } \\
\text { reviewed): }\end{array}$ & 0 & $\mathrm{x}$ & . & & $7 / 22 / 96$ \\
\hline & & & 5 & Test [Perform Data Review] button: & 0 & $\mathrm{x}$ & & & $7 / 22 / 96$ \\
\hline & & & 6 & Test [RADDETAIL] button: & 0 & $x$ & & & $7 / 22 / 96$ \\
\hline & & & 7 & Test [Return to Main 1221 Screen] button: & 0 & $\mathrm{x}$ & & & $7 / 22 / 96$ \\
\hline & & & 8 & Test [SOQTY] button: & 0 & $\mathrm{x}$ & & & $7 / 22 / 96$ \\
\hline & & & 9 & Test [List of Values] button for Iso Num field: & 0 & $\mathrm{x}$ & & & $7 / 22 / 96$ \\
\hline & & & 10 & Test Quantity field for formatting of value: & 0 & $x$ & & & $7 / 22 / 96$ \\
\hline & & & 11 & $\begin{array}{l}\text { Enter value for TMU field; test TMU field for } \\
\text { formatting of input: }\end{array}$ & 0 & $\mathrm{x}$ & & & $7 / 22 / 96$ \\
\hline & & & 12 & $\begin{array}{l}\text { Enter value for Alpha Ci field; test Alpha } \mathrm{Ci} \\
\text { field for formatting of input: }\end{array}$ & 0 & $\mathrm{x}$ & & & $7 / 22 / 96$ \\
\hline & & & 13 & $\begin{array}{l}\text { Processed Waste Data Review \& Mod. screen } \\
\text { (DMSS1221) }\end{array}$ & 0 & $\mathrm{x}$ & & & $7 / 22 / 96$ \\
\hline & & & 14 & $\begin{array}{l}\text { Enter value for Pu FGE field; test Pu FGE } \\
\text { fieid for formatting of input: }\end{array}$ & 0 & $x$ & & & $7 / 22 / 96$ \\
\hline & & & 15 & $\begin{array}{l}\text { Test [Return to Main } 1221 \text { Screen] button for } \\
\text { ISOQTY screen: }\end{array}$ & 0 & $\mathrm{x}$ & & & $7 / 22 / 96$ \\
\hline
\end{tabular}




\begin{tabular}{|c|c|c|c|c|c|c|c|c|c|}
\hline PROC \# & $\begin{array}{l}\text { PROCEDURE } \\
\text { NAME }\end{array}$ & $\underset{\#}{\mathrm{CASE}}$ & $\begin{array}{c}\text { STE } \\
\mathbf{P} \\
\# \\
\end{array}$ & STEP NAME & $\underset{\#}{\text { REV }}$ & $\begin{array}{l}\text { PASS } \\
\text { Ist } \\
\text { TEST }\end{array}$ & $\begin{array}{c}\text { PASS } \\
\text { RETEST }\end{array}$ & COMMENTS & $\begin{array}{c}\text { SIGNOF } \\
F \\
\text { DATE }\end{array}$ \\
\hline \multirow[t]{16}{*}{ DMS-F25 } & \multirow{16}{*}{$\begin{array}{l}\text { Processed Waste Data Review \& Mod. } \\
\text { screen (DMSS1221) }\end{array}$} & \multirow[t]{13}{*}{08} & 16 & Re-test [SSOQTY] button: & 0 & $\mathrm{x}$ & & & $7 / 22 / 96$ \\
\hline & & & 17 & Re-test [Return to Main 1221 Screen] button: & 0 & $\mathrm{x}$ & & & $7 / 22 / 96$ \\
\hline & & & 18 & Re-test [RADDETAIL] button: & 0 & $\mathrm{x}$ & & & $7 / 22 / 96$ \\
\hline & & & 19 & Re-test [Return to Main 1221 Screen] button: & 0 & $\mathrm{x}$ & & & $7 / 22 / 96$ \\
\hline & & & 20 & Test [HAZDETAIL] button: & 0 & $\mathrm{x}$ & & & $7 / 22 / 96$ \\
\hline & & & 21 & Test [CHEMCOMP] button: & 0 & $\mathrm{x}$ & & & $7 / 22 / 96$ \\
\hline & & & 22 & Test [Delete] button for CHEMCOMP records: & 0 & $\mathrm{x}$ & . & & $7 / 22 / 96$ \\
\hline & & & 23. & Test [Retum to Main 1221 Screen] button: & 0 & $\mathrm{x}$ & & & $7 / 22 / 96$ \\
\hline & & & 24 & Test [Perform Data Review] button: & 0 & $\mathrm{x}$ & & & $7 / 22 / 96$ \\
\hline & & & 25 & Test [Data Review Complete] button: & 0 & $\mathrm{x}$ & & & $7 / 22 / 96$ \\
\hline & & & 26 & Test [Exit] button: & 0 & $\mathrm{x}$ & & & $7 / 22 / 96$ \\
\hline & & & 27 & Exit the DMS system. & 0 & $\mathrm{x}$ & & & $7 / 22 / 96$ \\
\hline & & & 28 & Perform post-test query ' $f 25.08 \mathrm{q} \mathrm{a}^{\prime}$. & 0 & $\mathrm{x}$ & & & $7 / 22 / 96$ \\
\hline & & \multirow[t]{3}{*}{09} & 1 & Run pre-test query ' $25.09 \mathrm{q} \mathrm{b}$ '. & 0 & $\mathrm{x}$ & & & $7 / 22 / 96$ \\
\hline & & & 2 & $\begin{array}{l}\text { Logon to DMS system as a user (USERI) with } \\
\text { test role UPDATE privileges. }\end{array}$ & 0 & $\mathrm{x}$ & & & $7 / 22 / 96$ \\
\hline & & & 3 & $\begin{array}{l}\text { Select the Processed Waste Data Review \& } \\
\text { Mod. screen (DMSS1221) from the DMS Main } \\
\text { Menu: }\end{array}$ & 0 & $\mathrm{x}$ & & & $7 / 22 / 96$ \\
\hline
\end{tabular}


MS PHASE 1 TEST REPORT

PPENDIX B: FUNCTIONAL, TEST RESULTS
DOC NUMBER: HNF-SD-WM-TRP-287

REVISION:

DATE: $9 / 27 / 96$

\begin{tabular}{|c|c|c|c|c|c|c|c|c|c|}
\hline PROC \# & $\begin{array}{l}\text { PROCEDURE } \\
\text { NAME }\end{array}$ & $\underset{\#}{\text { CASE }}$ & $\begin{array}{c}\text { STE } \\
\mathbf{P} \\
\#\end{array}$ & STEP NAME & $\underset{\#}{\text { REV }}$ & $\begin{array}{c}\text { PASS } \\
\text { 1st } \\
\text { TEST }\end{array}$ & $\begin{array}{c}\text { PASS } \\
\text { RETEST }\end{array}$ & COMMENTS & $\begin{array}{c}\text { SIGNOF } \\
\text { F } \\
\text { DATE }\end{array}$ \\
\hline \multirow[t]{15}{*}{ DMS-F25 } & \multirow[t]{15}{*}{$\begin{array}{l}\text { Processed Waste Data Review \& Mod. } \\
\text { screen (DMSS1221) }\end{array}$} & \multirow[t]{15}{*}{09 . } & 4 & $\begin{array}{l}\text { Enter value for drum PIN (TRU RWM, not } \\
\text { reviewed): }\end{array}$ & 0 & $\mathrm{X}$ & & & $7 / 22 / 96$ \\
\hline & & & 5 & Test [Perform Data Review] button: & 1 & & $\mathrm{x}$ & & $8 / 20 / 96$ \\
\hline & & & 6 & Test [RADDETAIL] button: & 0 & $\mathrm{x}$ & & & $7 / 22 / 96$ \\
\hline & & & 7 & Enter value for Seal Number: & 0 & $\mathrm{x}$ & & & $7 / 22 / 96$ \\
\hline & & & 8 & $\begin{array}{l}\text { Test [List of Values] button for SWIMS } \\
\text { Category: }\end{array}$ & 0 & $\mathrm{x}$ & & & $7 / 22 / 96$ \\
\hline & & & 9 & $\begin{array}{l}\text { Test [List of Values] button for WRAP } \\
\text { Category: }\end{array}$ & 0 & $\mathrm{x}$ & & & $7 / 22 / 96$ \\
\hline & & & 10 & Enter value for Content Organic Volume \%: & 0 & $x$ & & . & $7 / 22 / 96$ \\
\hline & & & 11 & Test [Return to Main 1221 Screen] button: & 0 & $\mathrm{x}$ & & & $7 / 22 / 96$ \\
\hline & & & 12 & Test [HAZDETAIL] button: & 0 & $\mathrm{x}$ & & & $7 / 22 / 96$ \\
\hline & & & 13 & Change value for Waste Volume (m3): & 0 & $\mathrm{x}$ & & & $7 / 22 / 96$ \\
\hline & & & 14 & $\begin{array}{l}\text { Test [List of Values] button for Haz. Property } \\
\text { Code field: }\end{array}$ & 0 & $\mathrm{x}$ & . & & $7 / 22 / 96$ \\
\hline & & & 15 & Modify value for Designation Code: & 0 & $\mathrm{x}$ & & & $7 / 22 / 96$ \\
\hline & & & 16 & Test [List of Values] for Other Landban 1: & 0 & $\mathrm{x}$ & & & $7 / 22 / 96$ \\
\hline & & & 17 & Test [CHEMCOMP] button: & 0 & $\mathrm{X}$ & & & $7 / 22 / 96$ \\
\hline & & & 18 & Test direct entry of CHEMCOMP Id value: & 0 & $\mathrm{x}$ & & & $7 / 22 / 96$ \\
\hline
\end{tabular}




\begin{tabular}{|c|c|c|c|c|c|c|c|c|c|}
\hline PROC \# & $\begin{array}{l}\text { PROCEDURE } \\
\text { NAME }\end{array}$ & CASE & $\begin{array}{l}\text { STE } \\
\mathbf{P} \\
\#\end{array}$ & STEP NAME & $\underset{\#}{\mathbf{R E V}}$ & $\begin{array}{l}\text { PASS } \\
\text { 1st } \\
\text { TEST }\end{array}$ & $\begin{array}{l}\text { PASS } \\
\text { RETEST }\end{array}$ & COMMENTS & $\begin{array}{l}\text { SIGNOF } \\
\text { F } \\
\text { DATE }\end{array}$ \\
\hline \multirow[t]{16}{*}{ JMS-F25 } & \multirow[t]{16}{*}{$\begin{array}{l}\text { Processed Waste Data Review \& Mod. } \\
\text { soreen (DMSS1221) }\end{array}$} & \multirow[t]{8}{*}{09} & 19 & $\begin{array}{l}\text { Enter values for Weight }(\mathrm{kg}) \text { and Weight } \\
\text { Percent: }\end{array}$ & 0 & $x$ & & & $7 / 22 / 96$ \\
\hline & & & 20 & Enter new CHEMCOMP record: & 0 & $\mathrm{x}$ & & & $7 / 22 / 96$ \\
\hline & & & 21 & Test [Return to Main 1221 Screen] button: & 0 & $\mathrm{x}$ & & & $7 / 22 / 96$ \\
\hline & & & 22 & Test [Perform Data Review] button: & 0 & $\mathrm{x}$ & & & $7 / 22 / 96$ \\
\hline & & & 23 & Test [Data Review Complete] button: & 0 & $x$ & & & $7 / 22 / 96$ \\
\hline & & & 24 & Test [Exit] button: & 0 & $\mathbf{x}$ & & & $7 / 22 / 96$ \\
\hline & & & 25 & Exit the DMS system. & 0 : & $\mathrm{x}$ & & & $7 / 22 / 96$ \\
\hline & & & 26 & Perform post-test query ' $225.09 \mathrm{q}$ a'. & $\dot{0}$ & $\mathrm{x}$ & & & $7 / 22 / 96$ \\
\hline & & \multirow[t]{8}{*}{10} & 1 & Run pre-test query ' $25.10 \mathrm{q}$ b'. & 0 & $\mathrm{x}$ & & & $7 / 22 / 96$ \\
\hline & & & 2 & $\begin{array}{l}\text { Logon to DMS system as a user (USER1) with } \\
\text { test role UPDATE privileges. }\end{array}$ & 0 & $\mathrm{x}$ & & & $7 / 22 / 96$ \\
\hline & & & 3 & $\begin{array}{l}\text { Select the Processed Waste Data Review \& } \\
\text { Mod. screen (DMSS1221) from the DMS Main } \\
\text { Menu: }\end{array}$ & 0 & $\mathrm{x}$ & & & $7 / 22 / 96$ \\
\hline & & & 4 & Test [List of Values] button for drum PIN: & 0 & $\mathrm{x}$ & & & $7 / 22 / 96$ \\
\hline & & & 5 & Test [Perform Data Review] button: & 0 & $\mathrm{x}$ & & & $7 / 22 / 96$ \\
\hline & & & 6 & Test [WASTE] button: & 0 & $\mathrm{x}$ & & & $7 / 22 / 96$ \\
\hline & & & 7 & Enter value for Chemical Nature: & 0 & $\mathrm{x}$ & & & $7 / 22 / 96$ \\
\hline & & & 8 & Test List of Values for Routine field & 0 & $\mathrm{x}$ & & & $7 / 22 / 96$ \\
\hline
\end{tabular}




\begin{tabular}{|c|c|c|c|c|c|c|c|c|c|}
\hline PROC \# & $\begin{array}{l}\text { PROCEDURE } \\
\text { NAME }\end{array}$ & $\underset{\#}{\mathrm{CASE}}$ & $\begin{array}{c}\text { STE } \\
\mathbf{P} \\
\# \\
\end{array}$ & STEP NAME & $\underset{\#}{\mathrm{REV}}$ & $\begin{array}{c}\text { PASS } \\
\text { 1st } \\
\text { TEST } \\
\end{array}$ & $\begin{array}{c}\text { PASS } \\
\text { RETEST }\end{array}$ & COMMENTS & $\begin{array}{c}\text { SIGNOF } \\
\mathbf{F} \\
\text { DATE } \\
\end{array}$ \\
\hline \multirow[t]{16}{*}{ DMS-F25 } & \multirow{16}{*}{$\begin{array}{l}\text { Processed Waste Data Review \& Mod. } \\
\text { screen (DMSS1221) }\end{array}$} & \multirow[t]{16}{*}{10} & 9 & Enter value for Waste Description field: & 0 & $\mathrm{x}$ & & & $7 / 22 / 96$ \\
\hline & & & 10 & Test [Return to Main 1221 Screen] button: & 0 & $\mathrm{x}$ & & & $7 / 22 / 96$ \\
\hline & & & 11 & Test [Perform Data Review] button: & 0 & $\mathrm{x}$ & & & $7 / 22 / 96$ \\
\hline & & & 12 & Test [RADDETAIL] button: & .0 & $\mathrm{x}$ & & & $7 / 22 / 96$ \\
\hline & & & 13 & Test [Return to Main 1221 Screen] button: & 0 & $\mathrm{x}$ & & & $7 / 22 / 96$ \\
\hline & & & 14 & Test [ISOQTY] button: & 0 & $\mathrm{x}$ & & & $7 / 22 / 96$ \\
\hline & & & 15 & $\begin{array}{l}\text { Test up function key for Radioactive Isotope } \\
\text { Quantity (ISOQTY) Screen: }\end{array}$ & 0 & $x$ & & & $7 / 22 / 96$ \\
\hline & & & 16 & $\begin{array}{l}\text { Test }[<] \text { and }[>\text { ] buttons for Radioactive } \\
\text { Isotope Quantity (ISOQTY) Screen: }\end{array}$ & 0 & $\mathrm{x}$ & & & $7 / 22 / 96$ \\
\hline & & & 17 & Test Iso Num field for editability: & 0 & $\mathrm{x}$ & & . & $7 / 22 / 96$ \\
\hline & & & 18 & Test Quantity field for editability: & 0 & $\mathrm{x}$ & & & $7 / 22 / 96$ \\
\hline & & & 19 & Test TMU field for editability: & 0 & $x$ & & & $7 / 22 / 96$ \\
\hline & & & 20 & Test Alpha Ci field for editability: & 0 & $\mathrm{x}$ & & & $7 / 22 / 96$ \\
\hline & & & 21 & Test PE Ci field for editability: & 0 & $x$ & & & $7 / 22 / 96$ \\
\hline & & & 22 & Test Pu FGE field for editability: & 0 & $\mathrm{x}$ & & & $7 / 22 / 96$ \\
\hline & & & 23 & Test [Insert] button: & 0 & $\mathrm{x}$ & & & $7 / 22 / 96$ \\
\hline & & & 24 & Test [Return to Main 1221 Screen] button: & 1 & & $x$ & & $8 / 20 / 96$ \\
\hline
\end{tabular}




\begin{tabular}{|c|c|c|c|c|c|c|c|c|c|}
\hline PROC \# & $\begin{array}{l}\text { PROCEDURE } \\
\text { NAME }\end{array}$ & $\underset{\#}{\text { CASE }}$ & $\begin{array}{c}\text { STE } \\
\mathrm{P} \\
\# \\
\end{array}$ & STEP NAME & $\underset{\#}{\mathrm{REV}}$ & $\begin{array}{c}\text { PASS } \\
\text { 1st } \\
\text { TEST }\end{array}$ & $\begin{array}{c}\text { PASS } \\
\text { RETEST }\end{array}$ & COMMENTS & $\begin{array}{c}\text { SIGNOF } \\
F \\
\text { DATE }\end{array}$ \\
\hline \multirow[t]{14}{*}{ DMS-F25 } & \multirow{14}{*}{$\begin{array}{l}\text { Processed Waste Data Review \& Mod. } \\
\text { screen (DMSS1221) }\end{array}$} & \multirow[t]{14}{*}{10} & 25 & Test Iso Num field for direct entry: & 0 & $\mathrm{x}$ & & & $7 / 22 / 96$ \\
\hline & & & 26 & Test Quantity field for required entry: & 0 & $\mathrm{x}$ & & & $7 / 22 / 96$ \\
\hline & & & 27 & Test [Delete] button: & 0 & $\mathrm{x}$ & & & $7 / 22 / 96$ \\
\hline & & & 28 & $\begin{array}{l}\text { Test [Return to Main } 1221 \text { Screen] button for } \\
\text { ISOQTY screen: }\end{array}$ & 0 & $\mathrm{x}$ & & & $7 / 22 / 96$ \\
\hline & & & 29 & $\begin{array}{l}\text { Redisplay Radioactive Isotope Quantity } \\
\text { (ISOQTY) Record Screen: }\end{array}$ & 0 & $\mathrm{x}$ & & & $7 / 22 / 96$ \\
\hline & & & 30 & $\begin{array}{l}\text { Redisplay Radioactive Waste Container Detail } \\
\text { Record Screen: }\end{array}$ & 0 & $\mathrm{x}$ & & & $7 / 22 / 96$ \\
\hline & & & 31 & Test [Perform Data Review] button: & 0 & $\mathrm{x}$ & & . & $7 / 22 / 96$ \\
\hline & & & 32 & Test [PHYSCOMP] button: & 0 & $\mathrm{x}$ & & & $7 / 22 / 96$ \\
\hline & & & 33 & $\begin{array}{l}\text { Test up function key for Physical Component } \\
\text { Screen: }\end{array}$ & 0 & $\mathrm{x}$ & & & $7 / 22 / 96$ \\
\hline & & & 34 & $\begin{array}{l}\text { Test }[<] \text { and }[>] \text { buttons for Physical } \\
\text { Component Screen: }\end{array}$ & 0 & $\mathrm{x}$ & & & $7 / 22 / 96$ \\
\hline & & & 35 & Test Description field for editability: & 0 & $\mathrm{X}$ & & & $7 / 22 / 96$ \\
\hline & & & 36 & Test Volume Percent field for editability: & 0 & $\mathrm{X}$ & & & $7 / 22 / 96$ \\
\hline & & & 37 & Test Weight $(\mathrm{kg})$ field for editability: & 0 & $\mathrm{x}$ & & . & $7 / 22 / 96$ \\
\hline & & & 38 & $\begin{array}{l}\text { Test [lnsert] button for Physical Component } \\
\text { Screen: }\end{array}$ & 0 & $\mathrm{x}$ & & & $7 / 22 / 96$ \\
\hline
\end{tabular}




\begin{tabular}{|c|c|c|c|c|c|c|c|c|c|}
\hline PROC \# & $\begin{array}{l}\text { PROCEDURE } \\
\text { NAME }\end{array}$ & $\underset{\#}{\operatorname{CASE}}$ & $\begin{array}{c}\text { STE } \\
\mathbf{P} \\
\# \\
\end{array}$ & STEP NAME & $\underset{\#}{\mathrm{REV}}$ & $\begin{array}{l}\text { PASS } \\
\text { 1st } \\
\text { TEST }\end{array}$ & $\begin{array}{c}\text { PASS } \\
\text { RETEST }\end{array}$ & COMMENTS & $\begin{array}{c}\text { SIGNOF } \\
\text { F } \\
\text { DATE }\end{array}$ \\
\hline \multirow[t]{14}{*}{ DMS-F25 } & \multirow{14}{*}{$\begin{array}{l}\text { Processed Waste Data Review \& Mod. } \\
\text { screen (DMSS1221) }\end{array}$} & \multirow[t]{14}{*}{10} & 39 & Test entry for value for Description field: & 0 & $\mathrm{x}$ & & & $7 / 22 / 96$ \\
\hline & & & 40 & $\begin{array}{l}\text { Enter values for Volume Percent and Weight } \\
(\mathrm{kg}) \text { : }\end{array}$ & 0 & $\mathrm{x}$ & & & $7 / 22 / 96$ \\
\hline & & & 41 & $\begin{array}{l}\text { Test [Delete] button for Physical Component } \\
\text { Screen: }\end{array}$ & 0 & $\mathrm{x}$ & & & $7 / 22 / 96$ \\
\hline & & & $42 \cdots$ & Test [Return to Main 1221 Screen] button: & 0 & $\mathrm{X}$ & & & $7 / 22 / 96$ \\
\hline & & & 43 & Re-test [Retum to Main 1221 Screen] button: & 0 & $\mathrm{x}$ & & & $7 / 22 / 96$ \\
\hline & & & 44 & Redisplay Physical Component Screen: & 0 & $\mathrm{x}$ & & & $7 / 22 / 96$ \\
\hline & & & 45 & Test [HAZDETAIL] button: & 0 & $\mathrm{x}$ & & & $7 / 22 / 96$ \\
\hline & & & 46 & $\begin{array}{l}\text { Test Container Status field for enterability, } \\
\text { format, length, and case restriction: }\end{array}$ & 0 & $\mathrm{x}$ & & & $7 / 22 / 96$ \\
\hline & & & 47 & $\begin{array}{l}\text { Test Haz. Property Code field for format and } \\
\text { uniqueness of values: }\end{array}$ & 0 & $\mathrm{x}$ & & & $7 / 22 / 96$ \\
\hline & & & 48 & Test [DW_NUM] button: & 0 & $\mathrm{x}$ & & & $7 / 22 / 96$ \\
\hline & & & 49 & $\begin{array}{l}\text { Test down function key for Package Dangerous } \\
\text { Waste Numbers Record Screen: }\end{array}$ & 0 & $\mathrm{x}$ & & & $7 / 22 / 96$ \\
\hline & & & 50 & $\begin{array}{l}\text { Test }[<] \text { and }[>] \text { buttons for Package } \\
\text { Dangerous Waste Numbers Record Screen: }\end{array}$ & 0 & $x$ & & & $7 / 22 / 96$ \\
\hline & & & 51 & Test DW Number field for editability: & 0 & $\mathrm{x}$ & & & $7 / 22 / 96$ \\
\hline & & & 52 & Test Landban field for editability: & 0 & $x$ & & & $7 / 22 / 96$ \\
\hline
\end{tabular}




\begin{tabular}{|c|c|c|c|c|c|c|c|c|c|}
\hline PROC \# & $\begin{array}{l}\text { PROCEDURE } \\
\text { NAME }\end{array}$ & $\underset{\#}{\text { CASE }}$ & $\begin{array}{c}\text { STE } \\
P \\
\# \\
\end{array}$ & STEP NAME & $\begin{array}{c}\mathrm{REV} \\
\# .\end{array}$ & $\begin{array}{l}\text { PASS } \\
\text { 1st } \\
\text { TEST }\end{array}$ & $\begin{array}{c}\text { PASS } \\
\text { RETEST }\end{array}$ & COMMENTS & $\begin{array}{c}\text { SIGNOF } \\
\text { F } \\
\text { DATE } \\
\end{array}$ \\
\hline \multirow[t]{15}{*}{ MS-F25 } & \multirow{15}{*}{$\begin{array}{l}\text { Processed Waste Data Review \& Mod. } \\
\text { screen (DMSS1221) }\end{array}$} & \multirow[t]{15}{*}{10} & 53 & Test [Insert] button: & 0 & $\mathrm{x}$ & & & $7 / 22 / 96$ \\
\hline & & & 54 & Test DW Number field for direct entry: & 1 & & $\mathrm{x}$ & & $8 / 20 / 96$ \\
\hline & & & 55 & Test Landban field for required entry: & 0 & $\mathrm{x}$ & & & $7 / 22 / 96$ \\
\hline & & & 56 & Test [Delete] button: & 0 & $\mathrm{x}$ & & & $7 / 22 / 96$ \\
\hline & & & 57 & Test [Return to HAZDETAIL Screen] button: & 0 & $\mathrm{x}$ & & & $7 / 22 / 96$ \\
\hline & & & 58 & $\begin{array}{l}\text { Redisplay Package Dangerous Waste Numbers } \\
\text { Record Screen: }\end{array}$ & 0 & $\mathrm{x}$ & & & $7 / 22 / 96$ \\
\hline & & & 59 & Test [Return to Main 1221 Screen] button: & 0 & $\mathrm{x}$ & & & $7 / 22 / 96$ \\
\hline & & & 60 & Test [Perform Data Revjew] button: & 0 & $\mathrm{x}$ & & & $7 / 22 / 96$ \\
\hline & & & 61 & Test [CHEMCOMP] button: & 0 & $\mathrm{X}$ & & & $7 / 22 / 96$ \\
\hline & & & 62 & $\begin{array}{l}\text { Test up function key for Chemical Component } \\
\text { Screen: }\end{array}$ & 0 & $\mathrm{x}$ & & & $7 / 22 / 96$ \\
\hline & & & 63 & $\begin{array}{l}\text { Test }[<] \text { and }[>] \text { buttons for Chemical } \\
\text { Component Screen: }\end{array}$ & 0 & $\mathrm{x}$ & & & $7 / 22 / 96^{\circ}$ \\
\hline & & & 64 & Test ID field for editability: & 0 & $\mathrm{x}$ & & & $7 / 22 / 96$ \\
\hline & & & 65 & Test Weight $(\mathrm{kg})$ field for editability: & 0 & $x$ & & & $7 / 22 / 96$ \\
\hline & & & 66 & Test Weight Percent field for editability: & 0 & $\mathrm{x}$ & & & $7 / 22 / 96$ \\
\hline & & & 67 & $\begin{array}{l}\text { Test [nsert] button for Chemical Component } \\
\text { Screen: }\end{array}$ & 0 & $\mathrm{x}$ & & & $7 / 22 / 96$ \\
\hline
\end{tabular}




\begin{tabular}{|c|c|c|c|c|c|c|c|c|c|}
\hline PROC \# & $\begin{array}{l}\text { PROCEDURE } \\
\text { NAME }\end{array}$ & $\underset{\#}{\text { CASE }}$ & $\begin{array}{c}\text { STE } \\
\mathbf{P} \\
\#\end{array}$ & STEP NAME & $\underset{\#}{\operatorname{REV}}$ & $\begin{array}{l}\text { PASS } \\
\text { 1st } \\
\text { TEST }\end{array}$ & $\begin{array}{c}\text { PASS } \\
\text { RETEST }\end{array}$ & COMMENTS & $\begin{array}{c}\text { SIGNOF } \\
\text { D } \\
\text { DATE }\end{array}$ \\
\hline \multirow[t]{14}{*}{ JMS-F25 } & \multirow{14}{*}{$\begin{array}{l}\text { Processed Waste Data Review \& Mod. } \\
\text { screen (DMSS1221) }\end{array}$} & \multirow[t]{14}{*}{10} & 68 & Test entry for value for ID field: & 0 & $\mathrm{x}$ & & & $7 / 22 / 96$ \\
\hline & & & 69 & $\begin{array}{l}\text { Enter values for Weight }(\mathrm{kg}) \text { and Weight } \\
\text { Percent: }\end{array}$ & 0 & $\mathrm{x}$ & & & $7 / 22 / 96$ \\
\hline & & & 70 & $\begin{array}{l}\text { Test [Delete] button for Chemical Component } \\
\text { Screen: }\end{array}$ & 0 & $\mathrm{x}$ & & & $7 / 22 / 96$ \\
\hline & & & 71 & Test [Return to Main 1221 Screen] button: & 0 & $\mathrm{x}$ & & & $7 / 22 / 96$ \\
\hline & & & 72 & Re-test [Return to Main 1221 Screen] button: & 0 & $\mathrm{x}$ & & & $7 / 22 / 96$ \\
\hline & & & 73 & Redisplay Chemical Component Screen: & 0 & $\mathrm{x}$ & & & $7 / 22 / 96$ \\
\hline & & & 74 & Test [Perform Data Review] button: & 0 & $\mathrm{x}$ & & & $7 / 22 / 96$ \\
\hline & & & 75 & Test [APPMSDS] button & 0 & $\mathrm{x}$ & & & $7 / 22 / 96$ \\
\hline & & & 76 & $\begin{array}{l}\text { Test up function key for Applicable MSDS } \\
\text { Sereen: }\end{array}$ & 0 & $\mathrm{x}$ & & & $7 / 22 / 96$ \\
\hline & & & 77 & $\begin{array}{l}\text { Test }[<] \text { and }[>] \text { buttons for Applicable } \\
\text { MSDS Screen: }\end{array}$ & 0 & $\mathrm{x}$ & & & $7 / 22 / 96$ \\
\hline & & & 78 & Test MSDS ID field for editability: & 0 & $\mathrm{x}$ & & & $7 / 22 / 96$ \\
\hline & & & 79 & $\begin{array}{l}\text { Test [insert] button for Applicable MSDS } \\
\text { Screen: }\end{array}$ & 0 & $x$ & & & $7 / 22 / 96$ \\
\hline & & & 80 & Test entry for value for MSDS ID field: & 0 & $x$ & & & $7 / 22 / 96$ \\
\hline & & & 81 & $\begin{array}{l}\text { Test [Delete] button for Applicable MSDS } \\
\text { Screen: }\end{array}$ & 0 & $x$ & & . & $7 / 22 / 96$ \\
\hline
\end{tabular}




\begin{tabular}{|c|c|c|c|c|c|c|c|c|c|}
\hline PROC \# & $\begin{array}{l}\text { PROCEDURE } \\
\text { NAME }\end{array}$ & $\underset{\sharp}{\text { CASE }}$ & $\begin{array}{c}\text { STE } \\
\mathbf{P} \\
\# \\
\end{array}$ & STEP NAME & $\underset{\#}{\mathrm{REV}}$ & $\begin{array}{l}\text { PASS } \\
\text { 1st } \\
\text { TEST } \\
\end{array}$ & $\begin{array}{c}\text { PASS } \\
\text { RETEST }\end{array}$ & COMMENTS & $\begin{array}{c}\text { SIGNOF } \\
\text { F } \\
\text { DATE }\end{array}$ \\
\hline \multirow[t]{12}{*}{ DMS-F25 } & \multirow{12}{*}{$\begin{array}{l}\text { Processed Waste Data Review \& Mod. } \\
\text { screen (DMSS1221) }\end{array}$} & \multirow[t]{12}{*}{10} & 82 & Test [Return to Main 1221 Screen] button: & 0 & $\mathrm{x}$ & & & $7 / 22 / 96$ \\
\hline & & & 83 & Redisplay Applicable MSDS Screen: & 0 & $\mathrm{X}$ & & & $7 / 22 / 96$ \\
\hline & & & 84 & Test [Perform Data Review] button: & 0 & $\mathrm{x}$ & & & $7 / 22 / 96$ \\
\hline & & & 85 & $\begin{array}{l}\text { Test [List of Values] button for drum PIN } \\
\text { (default selection criteria 'LLW', 'Not } \\
\text { Reviewed'): }\end{array}$ & 0 & $\mathrm{x}$ & $\sim$ & & $7 / 22 / 96$ \\
\hline & & & 86 & $\begin{array}{l}\text { Test waste type selection criteria; test [List of } \\
\text { Values] button for 'TRU', 'Not Reviewed'): }\end{array}$ & 0 & $\mathrm{x}$ & & & $7 / 22 / 96$ \\
\hline & & & 87 & $\begin{array}{l}\text { Test waste type selection criteria; test [List of } \\
\text { Values] button for 'All', 'Not Reviewed'): }\end{array}$ & 0 & $\mathrm{x}$ & & & $7 / 22 / 96$ \\
\hline & & & 88 & $\begin{array}{l}\text { Test waste type selection criteria; test [List of } \\
\text { Values] button for 'LLW', 'Reviewed'): }\end{array}$ & 0 & $x$ & & & $7 / 22 / 96$ \\
\hline & & & 89 & $\begin{array}{l}\text { Test waste type selection criteria; test [List of } \\
\text { Values] button for 'TRU', 'Reviewed'): }\end{array}$ & 0 & $\mathrm{x}$ & & . & $7 / 22 / 96$ \\
\hline & & & 90 & $\begin{array}{l}\text { Test waste type selection criteria; test [List of } \\
\text { Values] button for 'TRU', 'Reviewed'): }\end{array}$ & 0 & $\mathrm{x}$ & & & $7 / 22 / 96$ \\
\hline & & & 91 & Test [Exit] button: & 0 & $\mathrm{x}$ & & & $7 / 22 / 96$ \\
\hline & & & 92 & Exit the DMS system. & 0 & $x$ & & & $7 / 22 / 96$ \\
\hline & & & 93 & Perform post-test query ' $25.10 \mathrm{q} \mathrm{a}^{\prime}$. & 0 & $\mathrm{X}$ & & & $7 / 22 / 96$ \\
\hline
\end{tabular}




\begin{tabular}{|c|c|c|c|c|c|c|c|c|c|}
\hline PROC \# & $\begin{array}{l}\text { PROCEDURE } \\
\text { NAME }\end{array}$ & $\underset{\#}{\text { CASE }}$ & $\begin{array}{l}\text { STE } \\
\mathbf{P} \\
\#\end{array}$ & STEP NAME & $\underset{\#}{\mathrm{REV}}$ & $\begin{array}{l}\text { PASS } \\
1 \text { șt } \\
\text { TEST }\end{array}$ & $\begin{array}{c}\text { PASS } \\
\text { RETEST }\end{array}$ & COMMENTS & $\begin{array}{c}\text { SIGNOF } \\
\text { F } \\
\text { DATE }\end{array}$ \\
\hline \multirow[t]{8}{*}{ DMS-F41 } & \multirow[t]{8}{*}{ Location Table screen (DMSS0452) } & \multirow[t]{8}{*}{01} & 1 & $\begin{array}{l}\text { Logon to DMS system as a user (USERI) with } \\
\text { test role UPDATE privileges. }\end{array}$ & 0 . & $\mathrm{x}$ & & . & $2 / 8 / 96$ \\
\hline & & & 2 & $\begin{array}{l}\text { Enter the Location Table screen (DMSS0452) } \\
\text { and perform the following tests. }\end{array}$ & 0 & $\mathrm{x}$ & & & $2 / 8 / 96$ \\
\hline & & & 3 & Test the $[>]$ and $[<]$ buttons. & 0 & $\mathrm{x}$ & & & $2 / 8 / 96$ \\
\hline & & & 4 & Test the $[>>]$ and $[<<]$ buttons. & 0 & $\mathrm{x}$ & & $:$ & $2 / 8 / 96$ \\
\hline & & & 5 & Test the [Insert] and [Commit] buttons. & 0 & $\mathrm{x}$ & & & $2 / 8 / 96$ \\
\hline & & & 6 & Test the [Delete] button. & 0 & $\mathrm{X}$ & & & $2 / 8 / 96$ \\
\hline & & & 7 & Test the [Exit] button & 0 & $\mathrm{x}$ & & & $2 / 8 / 96$ \\
\hline & & & 8 & Exit the DMS system. & 0 & $\mathrm{x}$ & & & $2 / 8 / 96$ \\
\hline \multirow[t]{6}{*}{ DMS-F42 } & \multirow{6}{*}{$\begin{array}{l}\text { DMSS0454 - Profile Isotopic Table. } \\
\text { screen }\end{array}$} & \multirow[t]{5}{*}{01} & 1 & $\begin{array}{l}\text { Login to DMS system as a user with system } \\
\text { administration privileges. }\end{array}$ & 0 & $\mathrm{x}$ & & & $2 / 8 / 96$ \\
\hline & & & 2 & $\begin{array}{l}\text { Choose "Profile Isotopic" from the "Table } \\
\text { Maint" pulldown on the DMS Main Menu. }\end{array}$ & 0 & $x$ & & & $2 / 8 / 96$ \\
\hline & & & 3 & Perform test cases & 0 & $\mathrm{x}$ & & & $2 / 8 / 96$ \\
\hline & & & 4 & $\begin{array}{l}\text { Verify performance of }[<],[>],[<<] \text {, and } \\
{[>>] \text { buttons in Profile table. }}\end{array}$ & 0 & $\mathrm{x}$ & & & 2/8/96 \\
\hline & & & 5 & $\begin{array}{l}\text { Verify performance of }[<],[>],[<<] \text {, and } \\
{[>>] \text { buttons in Profile Isotope table. }}\end{array}$ & 0 & $\mathrm{x}$ & & & $2 / 8 / 96$ \\
\hline & & 02 & 1 & $\begin{array}{l}\text { Verify [Insert] and [Delete] buttons on Profile } \\
\text { table. Insert will test the validation defined for } \\
\text { each freld in table. }\end{array}$ & 0 & $\mathrm{x}$ & & & $2 / 8 / 96$ \\
\hline
\end{tabular}




\begin{tabular}{|c|c|c|c|c|c|c|c|c|c|}
\hline PROC \# & $\begin{array}{l}\text { PROCEDURE } \\
\text { NAME }\end{array}$ & $\underset{\#}{\text { CASE }}$ & $\begin{array}{c}\text { STE } \\
\mathbf{P} \\
\# \\
\end{array}$ & STEP NAME & $\underset{\#}{\mathbf{R E V}}$ & $\begin{array}{l}\text { PASS } \\
\text { 1st } \\
\text { TEST }\end{array}$ & $\begin{array}{c}\text { PASS } \\
\text { RETEST }\end{array}$ & COMMENTS & $\begin{array}{c}\text { SIGNOF } \\
\text { F } \\
\text { DATE } \\
\end{array}$ \\
\hline DMS-F42 & $\begin{array}{l}\text { DMSS0454 - Profile Isotopic Table } \\
\text { screen }\end{array}$ & 03 & 1 & $\begin{array}{l}\text { Verify [Insert] and [Delete] buttons on Profile. } \\
\text { Isotopic table. Insert will test the validation } \\
\text { defined for each field in table. }\end{array}$ & 0 & $x$ & & & $2 / 8 / 96$ \\
\hline \multirow[t]{4}{*}{ DMS-F43 } & \multirow{4}{*}{$\begin{array}{l}\text { ROUTE DESCRIPTION TABLE } \\
\text { (DMSS0455) }\end{array}$} & \multirow[t]{4}{*}{01} & 1 & Log onto the DMS as USER1. & 0 & $x$ & & & $2 / 8 / 96$ \\
\hline & & & 2 & $\begin{array}{l}\text { Select "Tabie Maint and "Route desc" and } \\
\text { perform the following tests: }\end{array}$ & 0 & $\mathrm{X}$ & & & $2 / 8 / 96$ \\
\hline & & & 3 & insert a new record. & 0 & $\mathrm{x}$ & & & $2 / 8 / 96$ \\
\hline & & & 4 & Delete a record & 0 & $\mathrm{X}$ & & & $2 / 8 / 96$ \\
\hline \multirow[t]{4}{*}{ DMS-F44 } & \multirow[t]{4}{*}{$\begin{array}{l}\text { SHIP PICKLIST TYPE TABLE } \\
\text { (DMSS0456) }\end{array}$} & \multirow[t]{4}{*}{01} & 1 & $\begin{array}{l}\text { Log onto the DMS as USER1, and enter the } \\
\text { Shipment Pick List Type screen (DMSS0456) } \\
\text { and perform the following tests. }\end{array}$ & 0 & $\mathrm{x}$ & & & $2 / 8 / 96$ \\
\hline & & & 2 & Test the scroll buttons. & 0 & $\mathrm{x}$ & & & $2 / 8 / 96$ \\
\hline & & & 3 & Test the Insert button. & 0 & $\mathrm{x}$ & & & $2 / 8 / 96$ \\
\hline & & & 4 & Test the Delete button. & 0 & $\mathrm{x}$ & & & $2 / 8 / 96$ \\
\hline \multirow[t]{4}{*}{ DMS-F45 } & \multirow[t]{4}{*}{$\begin{array}{l}\text { SIE ISOTOPE NAME TABLE } \\
\text { (DMSS0457) }\end{array}$} & \multirow[t]{4}{*}{01} & 1 & $\begin{array}{l}\text { Log onto the DMS as USER1, and enter the } \\
\text { SIE Isotope Name Table screen (DMSSO457) } \\
\text { and perform the following tests. }\end{array}$ & 0 & $\mathrm{x}$ & . & & $2 / 8 / 96$ \\
\hline & & & 2 & Test the scroll buttons. & 0 & $\mathrm{x}$ & & & $2 / 8 / 96$ \\
\hline & & & 3 & Test the Insert button. & 0 & $x$ & & & $2 / 8 / 96$ \\
\hline & & & 4 & Test the Delete button. & 0 & $\mathrm{x}$ & & & $2 / 8 / 96$ \\
\hline
\end{tabular}




\begin{tabular}{|c|c|c|c|c|c|c|c|c|c|}
\hline PROC \# & $\begin{array}{l}\text { PROCEDURE } \\
\text { NAME }\end{array}$ & $\begin{array}{c}\text { CASE } \\
\#\end{array}$ & $\begin{array}{c}\text { STE } \\
\mathbf{P} \\
\sharp\end{array}$ & STEP NAME & $\underset{\#}{\text { REV }}$ & $\begin{array}{c}\text { PASS } \\
\text { Ist } \\
\text { TEST } \\
\end{array}$ & $\begin{array}{l}\text { PASS } \\
\text { RETEST }\end{array}$ & COMMENTS & $\begin{array}{c}\text { SIGNOF } \\
\text { F } \\
\text { DATE }\end{array}$ \\
\hline \multirow[t]{14}{*}{ DMS-F61 } & \multirow[t]{14}{*}{ Error Message Table Screen } & \multirow[t]{14}{*}{01} & 6 & $\begin{array}{l}\text { Produce error on atternpted edit of field } \\
\text { protected from update: }\end{array}$ & 0 & $\mathrm{x}$ & & & $2 / 8 / 96$ \\
\hline & & & 7 & Test [Commit] button: & 0 & $x$ & & & $2 / 8 / 96$ \\
\hline & & & 8 & $\begin{array}{l}\text { Test Execute Query menu function to display } \\
\text { current database table contents: }\end{array}$ & 0 & $\mathrm{x}$ & & & $2 / 8 / 96$ \\
\hline & & & 9 & Test $[>>]$ and $[<<]$ buttons: & 0 & $\mathrm{x}$ & & & $2 / 8 / 96$ \\
\hline & & & 10 & Test $[>]$ and $[<]$ buttons: & 0 & $\mathrm{x}$ & & & $2 / 8 / 96$ \\
\hline & & & 11 & $\begin{array}{l}\text { Enter simple query with criteria in multiple } \\
\text { data fields: }\end{array}$ & 0 & $\mathrm{x}$ & & & $2 / 8 / 96$ \\
\hline & & & 12 & Test [Delete] button: & 0 & $\mathrm{x}$ & & & $2 / 8 / 96$ \\
\hline & & & 13 & Commit changes: & 0 & $x$ & & & $2 / 8 / 96$ \\
\hline & & & 14 & $\begin{array}{l}\text { Test [Commit] button on incomplete record } \\
\text { insert: }\end{array}$ & 0 . & $\mathrm{x}$ & . & & $2 / 8 / 96$ \\
\hline & & & 15 & Test [Exit] button on incomplete record insert: & 0 & $\mathrm{x}$ & & & 2/8/96 \\
\hline & & & 16 & Test [Delete] button: & 0 & $\mathrm{x}$ & & & $2 / 8 / 96$ \\
\hline & & & 17 & $\begin{array}{l}\text { Test for prompt for commit of uncommitted } \\
\text { changes on Exit: }\end{array}$ & 0 & $\mathrm{x}$ & & & $2 / 8 / 96$ \\
\hline & & & 18 & Commit changes to database: & 0 & $\mathrm{x}$ & & & $2 / 8 / 96$ \\
\hline & & & 19 & Test [Exit] button & 0 & $\mathrm{x}$ & & & $2 / 8 / 96$ \\
\hline DMS-F62 & User Table Screen & 01 & 1 & $\begin{array}{l}\text { Choose "Admin", then "User table" from the } \\
\text { DMS Main Menu. }\end{array}$ & 0 & $\mathrm{x}$ & & & $2 / 8 / 96$ \\
\hline
\end{tabular}




\begin{tabular}{|c|c|c|c|c|c|c|c|c|c|}
\hline PROC \# & $\begin{array}{l}\text { PROCEDURE } \\
\text { NAME }\end{array}$ & $\underset{\#}{\mathrm{CASE}}$ & $\begin{array}{c}\text { STE } \\
\mathbf{P} \\
\#\end{array}$ & STEP NAME & $\underset{\#}{\text { REV }}$ & $\begin{array}{l}\text { PASS } \\
\text { 1st } \\
\text { TEST }\end{array}$ & $\begin{array}{c}\text { PASS } \\
\text { RETEST }\end{array}$ & COMMENTS & $\begin{array}{c}\text { SIGNOF } \\
\text { F } \\
\text { DATE }\end{array}$ \\
\hline \multirow[t]{13}{*}{ DMS-F62 } & \multirow[t]{13}{*}{ User Table Screen } & \multirow[t]{13}{*}{01} & 2 & $\begin{array}{l}\text { Perform the following SQA test cases to ensure } \\
\text { the proper screen is accessed and that it has the } \\
\text { proper attributes: }\end{array}$ & 0 & $\mathrm{x}$ & & & $2 / 8 / 96$. \\
\hline & & & 3 & $\begin{array}{l}\text { Test Count Query Hits menu function to } \\
\text { inventory current database table contents: }\end{array}$ & 0 & $\mathrm{x}$ & & & $2 / 8 / 96$ \\
\hline & & & 4 & $\begin{array}{l}\text { Test [nsert] button; test Usr Userid for } \\
\text { required entry; test length and case restriction } \\
\text { of Scrn Name field: }\end{array}$ & 0 & $\mathrm{x}$ & & & $2 / 8 / 96$ \\
\hline & & & 5 & $\begin{array}{l}\text { Test other Usr Account and Usr Bull Dt fields } \\
\text { for format, length, and case restriction: }\end{array}$ & 0 & & $\mathrm{x}$ & & $6 / 10 / 96$ \\
\hline & & & 6 & $\begin{array}{l}\text { Test format and case restriction of Usr Logon } \\
\text { Dt field: }\end{array}$ & 0 & & $\mathrm{x}$ & & $6 / 10 / 96$ \\
\hline & & & 7 & $\begin{array}{l}\text { Test format, length, and case restriction of Usr } \\
\text { Org and Usr Pers Id fieids: }\end{array}$ & 0 & $\mathrm{x}$ & & & $2 / 8 / 96$ \\
\hline & & & 8 & $\begin{array}{l}\text { Test format, length, and case restriction of Usr } \\
\text { Org and Usr Pers Id fields: }\end{array}$ & 0 & $\mathrm{x}$ & & & $2 / 8 / 96$ \\
\hline & & & 9 & Test Duplicate Record function key: & 0 & $\mathrm{x}$ & & & $2 / 8 / 96$ \\
\hline & & & 10 & Redisplay database table contents & 0 & $\mathrm{x}$ & & & $2 / 8 / 96$ \\
\hline & & & 11 & Test Usr Userid field for editability: & 0 & $\mathrm{x}$ & & & $2 / 8 / 96$ \\
\hline & & & 12 & Test Duplicate Field function key: & 0 & $\mathrm{x}$ & & & $2 / 8 / 96$ \\
\hline & & & 13 & Test $[>]$ button: & 0 & $\mathrm{x}$ & & & $2 / 8 / 96$ \\
\hline & & & 14 & Test $[>>]$ button: & 0 & $\mathrm{x}$ & & & $2 / 8 / 96$ \\
\hline
\end{tabular}




\begin{tabular}{|c|c|c|c|c|c|c|c|c|c|}
\hline PROC \# & $\begin{array}{l}\text { PROCEDURE } \\
\text { NAME }\end{array}$ & $\underset{\#}{\mathrm{CASE}}$ & $\begin{array}{l}\text { STE } \\
\mathbf{P} \\
\#\end{array}$ & STEP NAME & $\underset{\#}{\mathrm{REV}}$ & $\begin{array}{l}\text { PASS } \\
\text { 1st } \\
\text { TEST }\end{array}$ & $\begin{array}{c}\text { PASS } \\
\text { RETEST }\end{array}$ & COMMENTS & $\begin{array}{c}\text { SIGNOF } \\
\text { F } \\
\text { DATE } \\
\end{array}$ \\
\hline \multirow[t]{8}{*}{ DMS-F62 } & \multirow[t]{8}{*}{ User Table Screen } & \multirow[t]{8}{*}{01} & 15 & Test $[<]$ button: & 0 & $\mathrm{x}$ & & & $2 / 8 / 96$ \\
\hline & & & 16 & Test $[<<]$ button: & 0 & $\mathrm{x}$ & & & $2 / 8 / 96$ \\
\hline & & & 17 & Enter simple query: & 0 & $\mathrm{x}$ & & & $2 / 8 / 96$ \\
\hline & & & 18 & $\begin{array}{l}\text { Test [Commit] button with no uncommitted } \\
\text { database changes: }\end{array}$ & 0 & $\mathrm{x}$ & & & $2 / 8 / 96$ \\
\hline & & & 19 & Test [Delete] button: & 0 & $\mathrm{X}$ & & & $2 / 8 / 96$ \\
\hline & & & 20 & $\begin{array}{l}\text { Test for prompt for commit of uncommitted } \\
\text { changes on Exit: }\end{array}$ & 0 & $\mathrm{x}$ & & & $2 / 8 / 96$ \\
\hline & & & 21 & Commit changes to database: & 0 & $\mathrm{x}$ & . & & $2 / 8 / 96$ \\
\hline & & & 22 & Test [Exit] button & 0 & $\mathrm{x}$ & & . & $2 / 8 / 96$ \\
\hline \multirow[t]{5}{*}{ DMS-F63 } & \multirow[t]{5}{*}{ Role Table screen } & \multirow[t]{5}{*}{01} & 1. & $\begin{array}{l}\text { Choose "Admin", then "Role table" from the } \\
\text { DMS Main Menu. }\end{array}$ & 0 & $\mathrm{x}$ & & & $2 / 8 / 96$ \\
\hline & & & 3 & $\begin{array}{l}\text { Test [nsert] button; test length and case } \\
\text { restriction of Role Cd and Role Descr fields: }\end{array}$ & 0 & $\mathrm{x}$ & & & $2 / 8 / 96$ \\
\hline & & & 4 & Test [Commit] button: & 0 & $\mathrm{x}$ & & & $2 / 8 / 96$ \\
\hline & & & 5 & $\begin{array}{l}\text { Produce error on attempted edit of field } \\
\text { protected from update: }\end{array}$ & 0 & $\mathrm{x}$ & & & $2 / 8 / 96$ \\
\hline & & & 6 & Test [Commit] button: & 0 & $x$ & & & $2 / 8 / 96$ \\
\hline
\end{tabular}




\begin{tabular}{|c|c|c|c|c|c|c|c|c|c|}
\hline PROC \# & $\begin{array}{l}\text { PROCEDURE } \\
\text { NAME }\end{array}$ & $\underset{\#}{\text { CASE }}$ & $\begin{array}{c}\text { STE } \\
\mathrm{P} \\
\# \\
\end{array}$ & STEP NAME & $\underset{\#}{\text { REV }}$ & $\begin{array}{l}\text { PASS } \\
\text { 1st } \\
\text { TEST }\end{array}$ & $\begin{array}{c}\text { PASS } \\
\text { RETEST }\end{array}$ & COMMENTS & $\begin{array}{c}\text { SIGNOF } \\
\text { F } \\
\text { DATE } \\
\end{array}$ \\
\hline \multirow[t]{14}{*}{ DMS-F63 } & \multirow[t]{14}{*}{ Role Table screen } & \multirow[t]{9}{*}{01} & 7 & $\begin{array}{l}\text { Test Execute Query menu function to display } \\
\text { current database table contents: }\end{array}$ & 0 & $\mathrm{x}$ & & & $2 / 8 / 96$ \\
\hline & & & 8 & Test $[>>]$ button: & 0 & $\mathrm{x}$ & & & $2 / 8 / 96$ \\
\hline & & & 9 & Test $[<]$ button: & 0 & $\mathrm{x}$ & & & $2 / 8 / 96$ \\
\hline & & & 10 & $\begin{array}{l}\text { Test [Commit] button on incomplete record } \\
\text { insert: }\end{array}$ & 0 & $\mathrm{x}$ & & & $2 / 8 / 96$ \\
\hline & & & 11 & Test [Exit] button on incomplete record insert: & 0 & $\mathrm{x}$ & & & $2 / 8 / 96$ \\
\hline & & & 12 & Test [Delete] button: & 0 & $\mathrm{x}$ & & & $2 / 8 / 96$ \\
\hline & & & 13 & $\begin{array}{l}\text { Test for prompt for commit of uncommitted } \\
\text { changes on Exit: }\end{array}$ & 0 & $\mathrm{x}$ & & & $2 / 8 / 96$ \\
\hline & & & 14 & Commit changes to database: & 0 & $\mathrm{X}$ & & & $2 / 8 / 96$ \\
\hline & & & 15 & Test [Exit] button & 0 & $\mathrm{x}$ & & & $2 / 8 / 96$ \\
\hline & & \multirow[t]{5}{*}{02} & 1 & $\begin{array}{l}\text { Choose "Admin", then "Role table" from the } \\
\text { DMS Main Menu. }\end{array}$ & 0 & $\mathrm{x}$ & & & $2 / 8 / 96$ \\
\hline & & & 2 & $\begin{array}{l}\text { Perform the following SQA test cases to ensure } \\
\text { the proper screen is accessed and that it has the } \\
\text { proper attributes: }\end{array}$ & 0 & $\mathrm{x}$ & . & & $2 / 8 / 96$ \\
\hline & & & 3 & Test [Delete] button: & 0 & $\mathrm{x}$ & & & $2 / 8 / 96$ \\
\hline & & & 4 & $\begin{array}{l}\text { Prompt for commit of uncommitted records on } \\
\text { Exit: }\end{array}$ & 0 & $\mathrm{x}$ & & & $2 / 8 / 96$ \\
\hline & & & 5 & Compare database table to pre-test condition: & 0 & $\mathrm{x}$ & & & $2 / 8 / 96$ \\
\hline
\end{tabular}




\begin{tabular}{|c|c|c|c|c|c|c|c|c|c|}
\hline PROC \# & $\begin{array}{l}\text { PROCEDURE } \\
\text { NAME }\end{array}$ & $\underset{\#}{\text { CASE }}$ & $\begin{array}{l}\text { STE } \\
\mathbf{P} \\
\#\end{array}$ & STEP NAME & $\begin{array}{c}\text { REV } \\
\#\end{array}$ & $\begin{array}{l}\text { PASS } \\
\text { lst } \\
\text { TEST }\end{array}$ & $\begin{array}{l}\text { PASS } \\
\text { RETEST }\end{array}$ & COMMENTS & $\begin{array}{l}\text { SIGNOF } \\
\text { F } \\
\text { DATE }\end{array}$ \\
\hline DMS-F63 & Role Table screen & 02 & 6 & Test [Exit] button & 0 & $X$ & & & $2 / 8 / 96$ \\
\hline \multirow[t]{14}{*}{ DMS-F64 } & \multirow[t]{14}{*}{ Screen Table Screen } & \multirow[t]{14}{*}{01} & 1 & $\begin{array}{l}\text { Choose "Admin", then "Screen Table" from } \\
\text { the DMS Main Menu. }\end{array}$ & 0 & $x$ & & & $2 / 11 / 96$ \\
\hline & & & 2 & $\begin{array}{l}\text { Perform the following SQA test cases to ensure } \\
\text { the proper screen is accessed and that it has the } \\
\text { proper attributes: }\end{array}$ & 0 & $\mathrm{x}$ & & & $2 / 11 / 96$ \\
\hline & & & 3 & $\begin{array}{l}\text { Test Count Query Hits menu function to } \\
\text { inventory current database table contents: }\end{array}$ & 0 & $\mathrm{X}$ & & & $2 / 11 / 96$ \\
\hline & & & 4 & Insert new data records: & 0 & $\mathrm{X}$ & & & $2 / 11 / 96$ \\
\hline & & & 5 & Test Commit menu function: & 0 & $\mathrm{X}$ & & & $2 / 11 / 96$ \\
\hline & & & 6 & Edit an existing record: & 0 & $\mathbf{x}$ & & . & $2 / 11 / 96$ \\
\hline & & & 7 & Test Previous Field function key: & 0 & $\mathrm{X}$ & & & $2 / 11 / 96$ \\
\hline & & & 8 & Test [Commit] button: & 0 & $x$ & & & $2 / 11 / 96$ \\
\hline & & & 9 & $\begin{array}{l}\text { Test Execute Query menu function to display } \\
\text { current database table contents: }\end{array}$ & 0 & $\mathbf{X}$ & & & $2 / 11 / 96$ \\
\hline & & & 10 & Test Scroll Down and Scroll Up menu function & 0 & $X$ & & & $2 / 11 / 96$ \\
\hline & & & 11 & $\begin{array}{l}\text { Tèst Next Record and Previous Record menu } \\
\text { functions: }\end{array}$ & 0 & $\mathrm{X}$ & & & $2 / 11 / 96$ \\
\hline & & & 12 & Test $[>]$ and $[<]$ buttons: & 0 & $X$ & & & $2 / 11 / 96$ \\
\hline & & & 13 & Test $[>>]$ and $[<<]$ buttons: & 0 & $X$ & & & $2 / 11 / 96$ \\
\hline & & & 14 & Test Down and Up keys: & 0 & $\mathbf{X}$ & & & $2 / 11 / 96$ \\
\hline
\end{tabular}




\begin{tabular}{|c|c|c|c|c|c|c|c|c|c|}
\hline PROC \# & $\begin{array}{l}\text { PROCEDURE } \\
\text { NAME }\end{array}$ & $\underset{\#}{\mathrm{CASE}}$ & $\begin{array}{c}\text { STE } \\
\mathbf{P} \\
\# \\
\end{array}$ & STEP NAME & $\underset{\#}{\mathrm{REV}}$ & $\begin{array}{c}\text { PASS } \\
\text { 1st } \\
\text { TEST } \\
\end{array}$ & $\begin{array}{c}\text { PASS } \\
\text { RETEST }\end{array}$ & COMMENTS & $\begin{array}{c}\text { SIGNOF } \\
\mathbf{F} \\
\text { DATE }\end{array}$ \\
\hline \multirow[t]{12}{*}{ DMS-F64 } & \multirow[t]{12}{*}{ Screen Table Screen } & \multirow[t]{12}{*}{01} & 15 & Test Scroll Down and Scroll Up function keys: & 0 & $\mathrm{x}$ & & & $2 / 11 / 96$ \\
\hline & & & 16 & $\begin{array}{l}\text { Test Next Record and Previous Record (Page } \\
\text { Down and Page Up) function keys: }\end{array}$ & 0 & 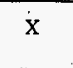 & & & $2 / 11 / 96$ \\
\hline & & & 17 & Test Enter Query function key: & 0 & $\mathrm{x}$ & & & $2 / 11 / 96$ \\
\hline & & & 18 & $\begin{array}{l}\text { Test Execute Query function key with simple } \\
\text { query criteria: }\end{array}$ & 0 & $\mathrm{x}$ & & & $2 / 11 / 96$ \\
\hline & & & 19 & Test user help function key: & 0 & & $\mathrm{x}$ & & $6 / 10 / 96$ \\
\hline & & & 20 & $\begin{array}{l}\text { Test Count Query Hits menu function to } \\
\text { inventory current database table contents: }\end{array}$ & 0 & $\mathrm{x}$ & & & $2 / 11 / 96$ \\
\hline & & & 21 & $\begin{array}{l}\text { Test field navigation and editing keys; edit an } \\
\text { existing record: }\end{array}$ & 0 & $\mathrm{x}$ & & & $2 / 11 / 96$ \\
\hline & & & 22 & $\begin{array}{l}\text { Test for prompt for commit of uncommitted } \\
\text { changes on Exit: }\end{array}$ & 0 & $\mathrm{x}$ & & & $2 / 11 / 96$ \\
\hline & & & 23 & $\begin{array}{l}\text { Choose "Admin", then "Screen Table" from } \\
\text { the DMS Main Menu. }\end{array}$ & 0 & $x$ & & & $2 / 11 / 96$ \\
\hline & & & 24 & $\begin{array}{l}\text { Perform the following SQA test cases to ensure } \\
\text { the proper screen is accessed and that it has the } \\
\text { proper attributes: }\end{array}$ & 0 & $\mathrm{x}$ & & & $2 / 11 / 96$ \\
\hline & & & 25 & $\begin{array}{l}\text { Test Count Query Hits menu function to } \\
\text { inventory current database table contents: }\end{array}$ & 0 & $x$ & & & $2 / 11 / 96$ \\
\hline & & & 26 & $\begin{array}{l}\text { Enter simple query with criteria in multiple } \\
\text { data fields: }\end{array}$ & 0 & $x$ & & & $2 / 11 / 96$ \\
\hline
\end{tabular}




\begin{tabular}{|c|c|c|c|c|c|c|c|c|c|}
\hline PROC \# & $\begin{array}{l}\text { PROCEDURE } \\
\text { NAME }\end{array}$ & $\underset{\#}{\text { CASE }}$ & $\begin{array}{c}\text { STE } \\
\mathbf{P} \\
\# \\
\end{array}$ & STEP NAME & $\underset{\#}{\mathrm{REV}}$ & $\begin{array}{c}\text { PASS } \\
\text { 1st } \\
\text { TEST } \\
\end{array}$ & $\begin{array}{l}\text { PASS } \\
\text { RETEST }\end{array}$ & COMMENTS & $\begin{array}{c}\text { SIGNOF } \\
\text { F } \\
\text { DATE } \\
\end{array}$ \\
\hline \multirow[t]{16}{*}{ DMS-F64 } & \multirow[t]{16}{*}{ Screen Table Screen } & \multirow[t]{16}{*}{01} & 27 & Test cancel query menu function: & 0 & $x$ & & & $2 / 11 / 96$ \\
\hline & & & 28 & Enter query mode, display last query criteria: & 0 & $x$ & & & $2 / 11 / 96$ \\
\hline & & & 29 & $\begin{array}{l}\text { Request count of records to be selected by } \\
\text { query; modify complex query criteria: }\end{array}$ & 0 & $\mathrm{x}$ & & & $2 / 11 / 96$ \\
\hline & & & 30 & Test Print Screen menu selection: & 0 & $\mathrm{x}$ & $\cdot$ & & $2 / 11 / 96$ \\
\hline & & & 31 & Execute entered query: & 0 & $\mathrm{x}$ & & & $2 / 11 / 96$ \\
\hline & & & 32 & Test Duplicate Record function key: & 0 & $\mathrm{x}$ & & & $2 / 11 / 96$ \\
\hline & & & 33 & Test Clear Field function key: & 0 & $\mathrm{x}$ & & & $2 / 11 / 96$ \\
\hline & & & 34 & $\begin{array}{l}\text { Retrieve contents of Windows clipboard (test } \\
\text { Clear Field vs. Cut functions): }\end{array}$ & 0 & $\mathrm{x}$ & & & $2 / 11 / 96$ \\
\hline & & & 35 & Test User Help menu function: & 0 & & $\mathrm{x}$ & & $6 / 10 / 96$ \\
\hline & & & 36 & $\begin{array}{l}\text { Test Previous Field function key and Copy } \\
\text { menu function: }\end{array}$ & 0 & $\mathrm{x}$ & & & $2 / 11 / 96$ \\
\hline & & & 37 & $\begin{array}{l}\text { Test Duplicate Field menu function at first } \\
\text { record: }\end{array}$ & 0 & $\mathrm{x}$ & & & $2 / 11 / 96$ \\
\hline & & & 38 & Test Commit function key: & 0 & $\mathrm{x}$ & & & $2 / 11 / 96$ \\
\hline & & & 39 & Test Clear Record function key: & 0 & $x$ & & & $2 / 11 / 96$ \\
\hline & & & 40 & Test Delete Record menu function: & 0 & $\mathrm{x}$ & & & $2 / 11 / 96$ \\
\hline & & & 41 & Commit changes: & 0 & $\mathrm{x}$ & & & $2 / 11 / 96$ \\
\hline & & & 42 & Test Delete Record function key: & 0 & $\mathrm{x}$ & & & $2 / 11 / 96$ \\
\hline
\end{tabular}




\begin{tabular}{|c|c|c|c|c|c|c|c|c|c|}
\hline PROC \# & $\begin{array}{l}\text { PROCEDURE } \\
\text { NAME }\end{array}$ & $\begin{array}{c}\text { CASE } \\
\#\end{array}$ & $\begin{array}{c}\text { STE } \\
\mathbf{P} \\
\#\end{array}$ & STEP NAME & $\underset{\#}{\text { REV }}$ & $\begin{array}{l}\text { PASS } \\
\text { 1st } \\
\text { TEST }\end{array}$ & $\begin{array}{l}\text { PASS } \\
\text { RETEST }\end{array}$ & COMMENTS & $\begin{array}{l}\text { SIGNOF } \\
\text { F } \\
\text { DATE }\end{array}$ \\
\hline \multirow[t]{9}{*}{ DMS-F64 } & \multirow[t]{9}{*}{ Screen Table Screen } & \multirow[t]{9}{*}{01} & 43 & Test Rollback menu function: & 0 & $\mathrm{x}$ & & & $2 / 11 / 96$ \\
\hline & & & 44 & Test Delete Record function key: & 0 & $\mathrm{X}$ & & & $2 / 11 / 96$ \\
\hline & & & 45 & $\begin{array}{l}\text { Request count of current database table } \\
\text { contents with prompt for commit of } \\
\text { uncommitted changes on Query function: }\end{array}$ & 0 & $X$ & & & $2 / 11 / 96$ \\
\hline & & & 46 & Display current database table contents: & 0 & $\mathrm{X}$ & & . & $2 / 11 / 96$ \\
\hline & & & 47 & Test Show Keys menu selection: & 0 & $\mathrm{X}$ & & & $2 / 11 / 96$ \\
\hline & & & 48 & Request DMS system information: & 0 & $\mathrm{X}$ & & & $2 / 11 / 96$ \\
\hline & & & 49 & Test Print Screen function key: & 0 & $\mathbf{X}$ & & & $2 / 11 / 96$ \\
\hline & & & 50 & $\begin{array}{l}\text { Test [Commit] button on incomplete record } \\
\text { insert: }\end{array}$ & 0 & $\mathrm{X}$ & & & $2 / 11 / 96$ \\
\hline & & & 51 & Test [Exit] button on incomplete record insert: & 0 & $\mathbf{x}$ & & & $2 / 11 / 96$ \\
\hline \multirow[t]{5}{*}{ DMS-F65 } & \multirow[t]{5}{*}{ User Role Table Screen } & \multirow[t]{5}{*}{01} & 1 & $\begin{array}{l}\text { Choose "Admin", then "User Role Table" } \\
\text { from the DMS Main Menu. }\end{array}$ & 0 & $X$ & & & $2 / 11 / 96$ \\
\hline & & & 2 & $\begin{array}{l}\text { Perform the following SQA test cases to ensure } \\
\text { the proper screen is accessed and that it has the } \\
\text { proper attributes: }\end{array}$ & 0 & $\mathrm{X}$ & & & $2 / 11 / 96$ \\
\hline & & & 3 & $\begin{array}{l}\text { Test Count Query Hits menu function to } \\
\text { inventory current database User table contents: }\end{array}$ & 0 & $\mathbf{X}$ & & & $2 / 11 / 96$ \\
\hline & & & 4 & $\begin{array}{l}\text { Test [Insert] button; test Users biock for entry } \\
\text { insert: }\end{array}$ & 0 & $\mathrm{X}$ & & & $2 / 11 / 96$ \\
\hline & & & 5 & Test Next Block menu function: & 0 & $\mathrm{X}$ & & & $2 / 11 / 96$ \\
\hline
\end{tabular}




\begin{tabular}{|c|c|c|c|c|c|c|c|c|c|}
\hline PROC \# & $\begin{array}{l}\text { PROCEDURE } \\
\text { NAME }\end{array}$ & $\underset{\#}{\text { CASE }}$ & $\begin{array}{c}\text { STE } \\
\mathbf{P} \\
\# \\
\end{array}$ & STEP NAME & $\underset{\#}{\text { REV }}$ & $\begin{array}{c}\text { PASS } \\
\text { Ist } \\
\text { TEST }\end{array}$ & $\begin{array}{c}\text { PASS } \\
\text { RETEST }\end{array}$ & COMMENTS & $\begin{array}{c}\text { SIGNOF } \\
\text { F } \\
\text { DATE }\end{array}$ \\
\hline \multirow[t]{14}{*}{ DMS-F65 } & \multirow[t]{14}{*}{ User Role Table Screen } & \multirow[t]{14}{*}{01} & 6 & $\begin{array}{l}\text { Test [Insert] button; test Userrole block for } \\
\text { entry insert: }\end{array}$ & 0 & $\mathrm{x}$ & & & $2 / 11 / 96$ \\
\hline & & & 7 & $\begin{array}{l}\text { Test Role field for format, length, case } \\
\text { restriction, and valid data value: }\end{array}$ & 0 & $\mathrm{x}$ & & & $2 / 11 / 96$ \\
\hline & & & 8 & Select Role from pop-up list: & 0 & $x$ & & & $2 / 11 / 96$ \\
\hline & & & 9 & $\begin{array}{l}\text { Test Description and Usr Name fields for } \\
\text { enterability: }\end{array}$ & 0 & $\mathrm{x}$ & & & $2 / 11 / 96$ \\
\hline & & & 10 & Test [List of Values] button: & 0 & $\mathrm{x}$ & & & $2 / 11 / 96$ \\
\hline & & & 11 & Test Valid Role Codes pop-up [Find] button: & 0 & $\mathrm{x}$ & & & $2 / 11 / 96$ \\
\hline & & & 12 & Test Valid Role Codes pop-up [Cancel] button: & 0 & $x$ & & & $2 / 11 / 96$ \\
\hline & & & 13 & $\begin{array}{l}\text { Test Valid Role Codes pop-up Scroll Down } \\
\text { function key: }\end{array}$ & 0 & $\mathrm{X}$ & & & $2 / 11 / 96$ \\
\hline & & & 14 & $\begin{array}{l}\text { Test Valid Role Codes pop-up Scroll Up } \\
\text { function key: }\end{array}$ & 0 & $x$ & & & $2 / 11 / 96$ \\
\hline & & & 15 & $\begin{array}{l}\text { Test Valid Role Codes pop-up Up, Down, } \\
\text { Right, and Left function keys: }\end{array}$ & 0 & $\mathrm{x}$ & & & $2 / 11 / 96$ \\
\hline & & & 16 & Test Description field autopopulate: & 0 & $\mathrm{x}$ & & & $2 / 11 / 96$ \\
\hline & & & 17 & $\begin{array}{l}\text { Test [Commit] button; test Role field for } \\
\text { uniqueness: }\end{array}$ & 0 & $x$ & & & $2 / 11 / 96$ \\
\hline & & & 18 & Test Clear Block menu function: & 0 & $\mathrm{x}$ & & & $2 / 11 / 96$ \\
\hline & & & 19 & $\begin{array}{l}\text { Test Count Query Hits menu function in } \\
\text { Userrole block; }\end{array}$ & 0 & $\mathrm{x}$ & & & $2 / 11 / 96$ \\
\hline
\end{tabular}




\begin{tabular}{|c|c|c|c|c|c|c|c|c|c|}
\hline PROC \# & $\begin{array}{l}\text { PROCEDURE } \\
\text { NAME }\end{array}$ & $\underset{\#}{\mathrm{CASE}}$ & $\begin{array}{c}\text { STE } \\
\mathbf{P} \\
\# \\
\end{array}$ & STEP NAME & $\underset{\#}{\mathrm{REV}}$ & $\begin{array}{c}\text { PASS } \\
\text { 1st } \\
\text { TEST }\end{array}$ & $\begin{array}{c}\text { PASS } \\
\text { RETEST }\end{array}$ & COMMENTS & $\begin{array}{c}\text { SIGNOF } \\
\text { F } \\
\text { DATE }\end{array}$ \\
\hline \multirow[t]{14}{*}{ DMS-F65 } & \multirow[t]{14}{*}{ User Role Table Screen } & \multirow[t]{14}{*}{01} & 20 & Test Previous Block menu selection: & 0 & $x$ & & - & $2 / 11 / 96$ \\
\hline & & & 21 & Test Usr Userid field for updatability: & 0 & $\mathrm{x}$ & & & $2 / 11 / 96$ \\
\hline & & & 22 & $\begin{array}{l}\text { Enter simple query on Usr Userid field; } \\
\text { request count of results: }\end{array}$ & 0 & $\mathrm{x}$ & & & $2 / 11 / 96$ \\
\hline & & & 23 & Enter complex query on Usr Userid field: & 0 & $\mathrm{x}$ & & & $2 / 11 / 96$ \\
\hline & & & 24 & $\begin{array}{l}\text { Test Previous Record and Next Block function } \\
\text { keys: }\end{array}$ & 0 & $x$ & & & $2 / 11 / 96$ \\
\hline & & & 25 & Redisplay database table records: & 0 & $\mathrm{x}$ & & & $2 / 11 / 96$ \\
\hline & & & 26 & Test $[>]$ and $[<]$ buttons in $U_{\text {sers block: }}$ & 0 & $\mathrm{x}$ & & & $2 / 11 / 96$ \\
\hline & & & 27 & Test $[<]$ button in Userrole block: & 0 & $x$ & & & $2 / 11 / 96$ \\
\hline & & & 28 & $\begin{array}{l}\text { Test for prompt for commit of uncommitted } \\
\text { changes on Exit: }\end{array}$ & 0 & $\mathrm{x}$ & & & $2 / 11 / 96$ \\
\hline & & & 29 & Test [Commit] button: & 0 & $\mathrm{x}$ & & & $2 / 11 / 96$ \\
\hline & & & 31 & Test [Delete] button: & 0 & $\mathrm{x}$ & & & $2 / 11 / 96$ \\
\hline & & & 32 & $\begin{array}{l}\text { Test for prompt for commit of uncommitted } \\
\text { changes on Exit: }\end{array}$ & 0 & $\mathrm{x}$ & & & $2 / 11 / 96$ \\
\hline & & & 33 & Commit changes to database: & 0 & $x$ & & & $2 / 11 / 96$ \\
\hline & & & 34 & Test [Exit] button & 0 & $\mathrm{x}$ & & & $2 / 11 / 96$ \\
\hline JMS-F66 & Role Screen Table Screen & 01 & 1 & $\begin{array}{l}\text { Choose "Admin", then "Role Screen Table" } \\
\text { from the DMS Main Menu. }\end{array}$ & 0 & $\mathrm{x}$ & & & $2 / 11 / 96$ \\
\hline
\end{tabular}




\begin{tabular}{|c|c|c|c|c|c|c|c|c|c|}
\hline PROC \# & $\begin{array}{l}\text { PROCEDURE } \\
\text { NAME }\end{array}$ & $\underset{\#}{\text { CASE }}$ & $\begin{array}{c}\text { STE } \\
\mathbf{P} \\
\# \\
\end{array}$ & STEP NAME & $\underset{\#}{\text { REV }}$ & $\begin{array}{c}\text { PASS } \\
\text { 1st } \\
\text { TEST }\end{array}$ & $\begin{array}{c}\text { PASS } \\
\text { RETEST }\end{array}$ & COMMENTS & $\begin{array}{c}\text { SIGNOF } \\
\text { F } \\
\text { DATE }\end{array}$ \\
\hline \multirow[t]{12}{*}{ DMS-F66 } & \multirow[t]{12}{*}{ Role Screen Table Screen } & \multirow[t]{12}{*}{01} & 2 & $\begin{array}{l}\text { Perform the following SQA test cases to ensure } \\
\text { the proper screen is accessed and that it has the } \\
\text { proper attributes: }\end{array}$ & 0 & $\mathrm{x}$ & & & $2 / 11 / 96$ \\
\hline & & & 3 & $\begin{array}{l}\text { Test Count Query Hits menu function to } \\
\text { inventory current database Role table contents: }\end{array}$ & 0 & $\mathrm{x}$ & & & $2 / 11 / 96$ \\
\hline & & & 4 & $\begin{array}{l}\text { Test [nsert] button; test Role block for entry } \\
\text { insert: }\end{array}$ & 0 & $\mathrm{x}$ & & & $2 / 11 / 96$ \\
\hline & & & 5 & Test Next Block menu function: & 0 & $\mathrm{x}$ & . & & $2 / 11 / 96$ \\
\hline & & & 6 & $\begin{array}{l}\text { Test Count Query Hits menu function to } \\
\text { inventory current database Rolescreen table } \\
\text { contents for Role 'ENGDEV': }\end{array}$ & 0 & $\mathrm{x}$ & & & $2 / 11 / 96$ \\
\hline & & & 7 & $\begin{array}{l}\text { Test [insert] button; test Rolescreen block for } \\
\text { entry insert: }\end{array}$ & 0 & $\mathrm{x}$ & & & $2 / 11 / 96$ \\
\hline & & & 8 & $\begin{array}{l}\text { Test Rscrn Scrn field for format, length, case } \\
\text { restriction, and valid data value: }\end{array}$ & 0 & $\mathrm{x}$ & & & $2 / 11 / 96$ \\
\hline & & & 9 & Select Screen from pop-up list: & 0 & $\mathrm{x}$ & & & $2 / 11 / 96$ \\
\hline & & & 10 & $\begin{array}{l}\text { Test } \mathrm{Rsc} \text { freld for format, length, case } \\
\text { restriction, and valid data value: }\end{array}$ & 0 & $\mathrm{x}$ & & i & $2 / 11 / 96$ \\
\hline & & & 11 & Test Role Descr field for enterability: & 0 & $\dot{x}$ & & & $2 / 11 / 96$ \\
\hline & & & 12 & Test Role Cd field for editability: & 0 & $\mathrm{x}$ & & & $2 / 11 / 96^{\circ}$ \\
\hline & & & 13 & $\begin{array}{l}\text { Test [Commit] button; test Rscrn Scrn field for } \\
\text { uniqueness: }\end{array}$ & 0 & $\mathrm{x}$ & & & $2 / 11 / 96$ \\
\hline
\end{tabular}




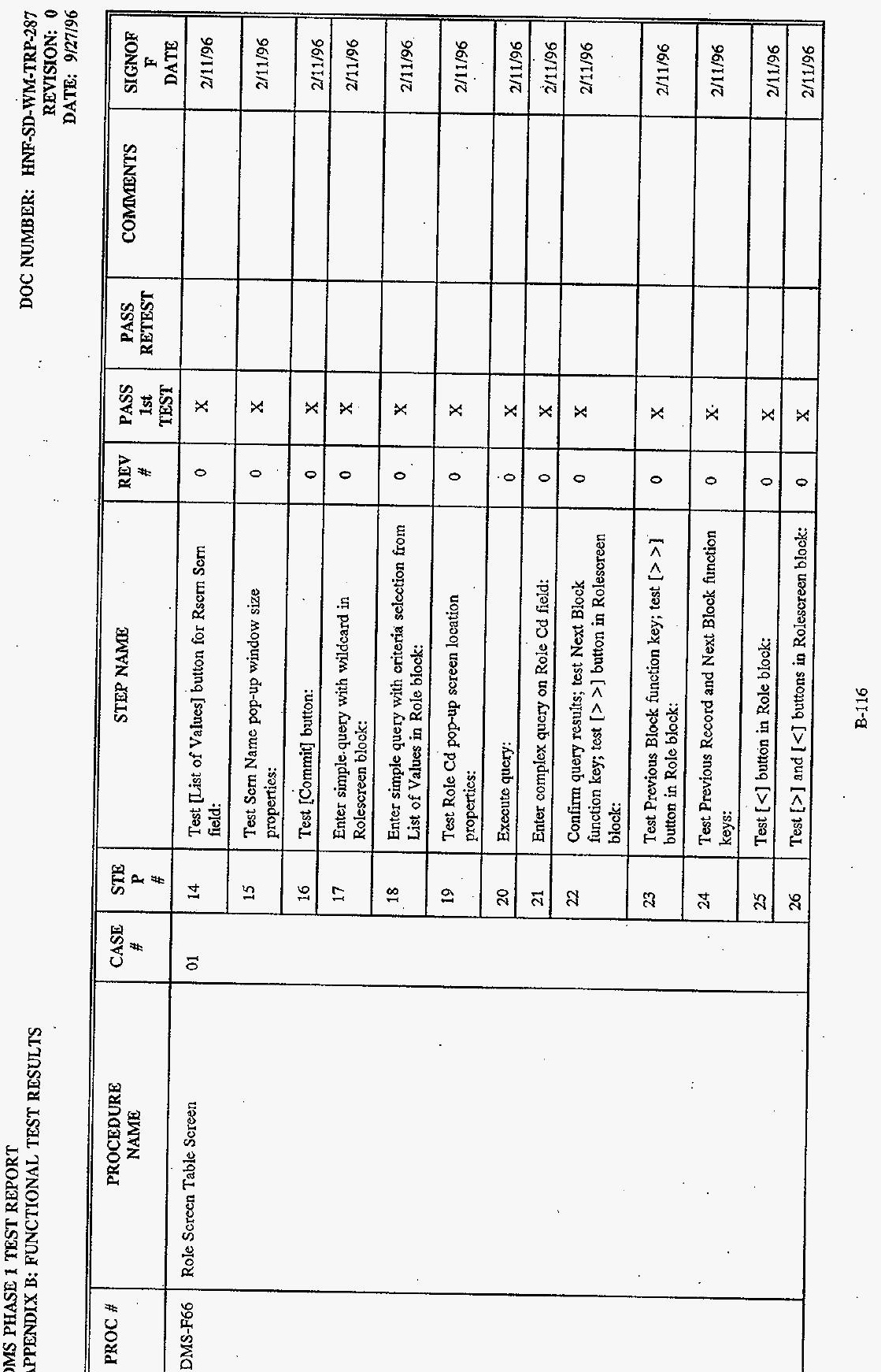




\begin{tabular}{|c|c|c|c|c|c|c|c|c|c|c|}
\hline PROC \# & $\begin{array}{l}\text { PROCEDURE } \\
\text { NAME }\end{array}$ & & $\underset{\#}{\mathrm{CASE}}$ & $\begin{array}{c}\text { STE } \\
\mathbf{P} \\
\# \\
\end{array}$ & STEP NAME & $\underset{\#}{\text { REV }}$ & $\begin{array}{c}\text { PASS } \\
\text { Ist } \\
\text { TEST }\end{array}$ & $\begin{array}{c}\text { PASS } \\
\text { RETEST }\end{array}$ & COMMENTS & $\begin{array}{c}\text { SIGNOF } \\
\text { F } \\
\text { DATE }\end{array}$ \\
\hline \multirow[t]{4}{*}{ DMS-F66 } & \multirow[t]{4}{*}{ Role Screen Table Screen } & & \multirow[t]{4}{*}{01} & 27 & Test [Delete] button: & 0 & $\mathrm{x}$ & & & $2 / 11 / 96$ \\
\hline & & & & 28 & $\begin{array}{l}\text { Test for prompt for commit of uncommitted } \\
\text { changes on request of query hits: }\end{array}$ & 0 & $\mathrm{x}$ & & & $2 / 11 / 96$ \\
\hline & & & & 29 & Request count of database Role records & 0 & $x$ & & & $2 / 11 / 96$ \\
\hline & & & & 30 & Test [Exit] button & 0 & $\mathrm{x}$ & & & $2 / 11 / 96$ \\
\hline \multirow[t]{7}{*}{ DMS-F81 } & \multirow[t]{7}{*}{ System Security } & \multirow{7}{*}{. } & \multirow[t]{7}{*}{01} & 1 & need to reload: inadvertent delete & 0 & OPEN & & & \\
\hline & & & & 2 & $\begin{array}{l}\text { Enter the Receiving screen and verify that } \\
\text { query for data is allowed but commit of data to } \\
\text { the database is not. }\end{array}$ & 0 & OPEN & . & & - \\
\hline & & & & 3 & $\begin{array}{l}\text { Enter the NDE screen and verify that query for } \\
\text { data is allowed but commit of data to the } \\
\text { database is not. }\end{array}$ & 0 & OPEN & & & \\
\hline & & & & 4 & $\begin{array}{l}\text { Enter the NDA screen and verify that query } \\
\text { for data is allowed but commit of data to the } \\
\text { database is not. }\end{array}$ & 0 & OPEN & & & \\
\hline & & & & 5 & $\begin{array}{l}\text { Enter the LLW Drum Status at Entry screen } \\
\text { and verify that query for data is allowed but } \\
\text { commit of data to the database is not. }\end{array}$ & 0 & OPEN & & & \\
\hline & & & & 6 & $\begin{array}{l}\text { Enter the LLW Sorting Table screen and verify } \\
\text { that query for data is allowed but commit of } \\
\text { data to the database is not. }\end{array}$ & 0 & OPEN & & & \\
\hline & & & & 7 & $\begin{array}{l}\text { Attempt to enter the LLW Exit Glovebox } \\
\text { screen and verify that query for data is allowed } \\
\text { but commit of data to the database is not. }\end{array}$ & 0 & OPEN & & & \\
\hline
\end{tabular}




\begin{tabular}{|c|c|c|c|c|c|c|c|c|c|}
\hline PROC \# & $\begin{array}{l}\text { PROCEDURE } \\
\text { NAME }\end{array}$ & $\underset{\#}{\text { CASE }}$ & $\begin{array}{c}\text { STE } \\
\text { P } \\
\# \\
\end{array}$ & STEP NAME & $\underset{\sharp}{\mathrm{REV}}$ & $\begin{array}{c}\text { PASS } \\
\text { 1st } \\
\text { TEST }\end{array}$ & $\begin{array}{c}\text { PASS } \\
\text { RETEST }\end{array}$ & COMMENTS & $\begin{array}{c}\text { SIGNOF } \\
\text { F } \\
\text { DATE }\end{array}$ \\
\hline \multirow[t]{8}{*}{ DMS-F81 } & \multirow[t]{8}{*}{ System Security } & \multirow[t]{8}{*}{01} & 8 & $\begin{array}{l}\text { Enter the Loading Dock Container Shipping } \\
\text { screen and verify that query for data is allowed } \\
\text { but commit of data to the database is not. }\end{array}$ & 0 & OPEN & & & \\
\hline & & & 9 & $\begin{array}{l}\text { Enter the Processing List Additions sereen and } \\
\text { verify that query for data is allowed but } \\
\text { commit of data to the database is not. }\end{array}$ & 0 & OPEN & & & \\
\hline & & & 10 & $\begin{array}{l}\text { Enter the Process List screen and verify that } \\
\text { query for data is allowed but commit of data to } \\
\text { the database is not. }\end{array}$ & 0 & OPEN & & & \\
\hline & & & 11 & $\begin{array}{l}\text { Enter the AS/RS Storage Pick List screen and } \\
\text { verify that query for data is allowed but } \\
\text { commit of data to the database is not. }\end{array}$ & 0 & OPEN & & & \\
\hline & & & 12 & $\begin{array}{l}\text { Enter the AS/RS Shipping Pick List screen and } \\
\text { verify that query for data is allowed but } \\
\text { commit of data to the database is not. }\end{array}$ & 0 & OPEN & & . & \\
\hline & & & 13 & $\begin{array}{l}\text { Enter the Radiological Inventory Summary } \\
\text { soreen and verify that query for data is allowed } \\
\text { but commit of data to the database is not. }\end{array}$ & 0 & OPEN & & & \\
\hline & & & 14 & $\begin{array}{l}\text { Enter the Processed Waste Data } \\
\text { Review/Modification screen and verify that } \\
\text { query for data is allowed but commit of data to } \\
\text { the database is not. }\end{array}$ & 0 & OPEN & & & \\
\hline & & & 15 & $\begin{array}{l}\text { Enter the Location Table screen and verify that } \\
\text { query for data is allowed but commit of data to } \\
\text { the database is not. }\end{array}$ & 0 & OPEN & & & \\
\hline
\end{tabular}




\begin{tabular}{|c|c|c|c|c|c|c|c|c|c|}
\hline PROC \# & $\begin{array}{l}\text { PROCEDURE } \\
\text { NAME }\end{array}$ & $\underset{\#}{\text { CASE }}$ & $\begin{array}{c}\text { STE } \\
\mathbf{P} \\
\# \\
\end{array}$ & STEP NAME & $\underset{\#}{\mathrm{REV}}$ & $\begin{array}{c}\text { PASS } \\
\text { 1st } \\
\text { TEST }\end{array}$ & $\begin{array}{c}\text { PASS } \\
\text { RETEST }\end{array}$ & COMMENTS & $\begin{array}{c}\text { SIGNOF } \\
F \\
\text { DATE } \\
\end{array}$ \\
\hline \multirow[t]{8}{*}{ DMS-F81 } & \multirow[t]{8}{*}{ System Security } & \multirow[t]{8}{*}{01} & 16 & $\begin{array}{l}\text { Enter the Profile Isotopic screen and verify that } \\
\text { query for data is allowed but commit of data to } \\
\text { the database is not. }\end{array}$ & 0 & OPEN & & & \\
\hline & & & 17 & $\begin{array}{l}\text { Enter the Route Description Table screen and } \\
\text { verify that query for data is allowed but } \\
\text { commit of data to the database is not. }\end{array}$ & 0 & OPEN & & & \\
\hline & & & 18 & $\begin{array}{l}\text { Enter the Shipping Pick List Type Table screen } \\
\text { and verify that query for data is allowed but } \\
\text { commit of data to the database is not. }\end{array}$ & 0 & OPEN & $\therefore$ & & \\
\hline & & & 19 & $\begin{array}{l}\text { Enter the SIE Isotopic Name Table screen and } \\
\text { verify that query for data is allowed but. } \\
\text { commit of data to the database is not. }\end{array}$ & 0 & OPEN & & & \\
\hline & & & 20 & $\begin{array}{l}\text { Enter the TRU Container Code Table screen } \\
\text { and verify that query for data is allowed but } \\
\text { commit of data to the database is not. }\end{array}$ & 0 & OPEN & & & \\
\hline & & & 21 & $\begin{array}{l}\text { Enter the Fieid Analysis Type Table screen and } \\
\text { verify that query for data is allowed but } \\
\text { commit of data to the database is not. }\end{array}$ & 0 & OPEN & & . & \\
\hline & & & 22 & $\begin{array}{l}\text { Enter the Material Table screen and verify that } \\
\text { query for data is allowed but commit of data to } \\
\text { the database is not. }\end{array}$ & 0 & OPEN & & & . \\
\hline & & & 23 & $\begin{array}{l}\text { Enter the Wrap Miscelianeous Code Table } \\
\text { screen and verify that query for data is allowed } \\
\text { but commit of data to the database is not. }\end{array}$ & 0 & OPEN & & & \\
\hline
\end{tabular}




\begin{tabular}{|c|c|c|c|c|c|c|c|c|c|}
\hline PROC \# & $\begin{array}{l}\text { PROCEDURE } \\
\text { NAME }\end{array}$ & $\underset{\sharp}{\text { CASE }}$ & $\begin{array}{c}\text { STE } \\
\mathrm{P} \\
\sharp \\
\end{array}$ & STEP NAME & $\underset{\#}{\operatorname{REV}}$ & $\begin{array}{l}\text { PASS } \\
\text { Ist } \\
\text { TEST }\end{array}$ & $\begin{array}{c}\text { PASS } \\
\text { RETEST }\end{array}$ & COMMENTS & $\begin{array}{c}\text { SIGNOF } \\
\text { F } \\
\text { DATE }\end{array}$ \\
\hline \multirow[t]{8}{*}{ DMS-F81 } & \multirow[t]{8}{*}{ System Security } & \multirow[t]{8}{*}{01} & 24 & $\begin{array}{l}\text { Enter the Laboratory Analysis Table screen } \\
\text { and verify that query for data is allowed but } \\
\text { commit of data to the database is not. }\end{array}$ & 0 & OPEN & & & \\
\hline & & & 25 & $\begin{array}{l}\text { Enter the Sample Container Table screen and } \\
\text { verify that query for data is allowed but } \\
\text { commit of data to the database is not. }\end{array}$ & 0 & OPEN & $\because$ & & \\
\hline & & & 26 & $\begin{array}{l}\text { Enter the Sample Matrix Table screen and } \\
\text { verify that query for data is allowed but } \\
\text { commit of data to the database is not. }\end{array}$ & 0 & OPEN & & & \\
\hline & & & 27 & $\begin{array}{l}\text { Enter the Sampling Method Table screen and } \\
\text { verify that query for data is allowed but } \\
\text { commit of data to the database is not. }\end{array}$ & 0 & OPEN & & & \\
\hline & & & 28 & $\begin{array}{l}\text { Enter the Treatment Procedure Table screen } \\
\text { and verify that query for data is allowed but } \\
\text { commit of data to the database is not. }\end{array}$ & 0 & OPEN & & & \\
\hline & & & 29 & $\begin{array}{l}\text { Enter the Error Message Table screen and } \\
\text { verify that query for data is allowed but } \\
\text { commit of data to the database is not. }\end{array}$ & 0 & OPEN & & & \\
\hline & & & 30 & $\begin{array}{l}\text { Enter the User Table screen and verify that } \\
\text { query for data is allowed but commit of data to } \\
\text { the database is not. }\end{array}$ & 0 & OPEN & & & \\
\hline & & & 31 & $\begin{array}{l}\text { Enter the Role Table screen and verify that } \\
\text { query for data is allowed but commit of data to } \\
\text { the database is not. }\end{array}$ & 0 & OPEN & & & \\
\hline
\end{tabular}




\begin{tabular}{|c|c|c|c|c|c|c|c|c|c|}
\hline PROC \# & $\begin{array}{l}\text { PROCEDURE } \\
\text { NAME }\end{array}$ & $\begin{array}{c}\text { CASE } \\
\#\end{array}$ & $\begin{array}{c}\text { STE } \\
\mathbf{P} \\
\#\end{array}$ & STEP NAME & $\underset{\#}{\text { REV }}$ & $\begin{array}{l}\text { PASS } \\
\text { Ist } \\
\text { TEST }\end{array}$ & $\begin{array}{l}\text { PASS } \\
\text { RETEST }\end{array}$ & COMMENTS & $\begin{array}{l}\text { SIGNOF } \\
\text { F } \\
\text { DATE }\end{array}$ \\
\hline \multirow[t]{10}{*}{ DMS-F81 } & \multirow[t]{10}{*}{ System Security } & \multirow[t]{3}{*}{01} & 32 & $\begin{array}{l}\text { Enter the Screen Table screen and verify that } \\
\text { query for data is allowed but commit of data to } \\
\text { the database is not. }\end{array}$ & 0 & OPEN & & & . \\
\hline & & & 33 & $\begin{array}{l}\text { Enter the User Role Table screen and verify } \\
\text { that query for data is allowed but commit of } \\
\text { data to the database is not. }\end{array}$ & 0 & OPEN & & & \\
\hline & & & 34 & $\begin{array}{l}\text { Enter the Role Screen Table screen and verify } \\
\text { that query for data is allowed but commit of } \\
\text { data to the database is not. }\end{array}$ & 0 & OPEN & & & \\
\hline & & \multirow[t]{7}{*}{02} & 1 & $\begin{array}{l}\text { Logon to DMS system as a user with test role } \\
\text { NO-ACC privileges and verify the DMS Main } \\
\text { Menu screen appears. }\end{array}$ & 0 & OPEN & & & \\
\hline & & & 2 & $\begin{array}{l}\text { Attempt to enter the Receiving screen and } \\
\text { verify no access allowed. }\end{array}$ & 0 & OPEN & & & \\
\hline & & & 3 & $\begin{array}{l}\text { Attempt to enter the Bar Code Generator } \\
\text { screen and verify no access allowed. }\end{array}$ & 0 & OPEN & & & \\
\hline & & & 4 & $\begin{array}{l}\text { Attempt to enter the NDE screen and verify no } \\
\text { access allowed. }\end{array}$ & 0 & OPEN & & & \\
\hline & & & 5 & $\begin{array}{l}\text { Attempt to enter the NDA screen and verify no } \\
\text { access allowed. }\end{array}$ & 0 & OPEN & & & \\
\hline & & & 6 & $\begin{array}{l}\text { Attempt to enter the LLW Drum Status at } \\
\text { Entry screen and verify no access allowed. }\end{array}$ & 0 & OPEN & & & \\
\hline & & & 7 & $\begin{array}{l}\text { Attempt to enter the LLW Sorting Table screen } \\
\text { and verify no access allowed. }\end{array}$ & 0 & OPEN & & & \\
\hline
\end{tabular}




\begin{tabular}{|c|c|c|c|c|c|c|c|c|c|}
\hline PROC \# & $\begin{array}{l}\text { PROĊEDURE } \\
\text { NAME }\end{array}$ & $\underset{\#}{\text { CASE }}$ & $\begin{array}{c}\text { STE } \\
\mathbf{P} \\
\# \\
\end{array}$ & STEP NAME & $\underset{\#}{\text { REV }}$ & $\begin{array}{c}\text { PASS } \\
\text { 1st } \\
\text { TEST } \\
\end{array}$ & $\begin{array}{c}\text { PASS } \\
\text { RETEST }\end{array}$ & COMMENTS & $\begin{array}{c}\text { SIGNOF } \\
\text { F } \\
\text { DATE }\end{array}$ \\
\hline \multirow[t]{11}{*}{ DMS-F81 } & \multirow[t]{11}{*}{ System Security } & \multirow[t]{11}{*}{02} & 8 & $\begin{array}{l}\text { Attempt to enter the LLW Exit Glovebox } \\
\text { screen and verify no access allowed. }\end{array}$ & 0 & OPEN & & & \\
\hline & & & 9 & $\begin{array}{l}\text { Attempt to enter the Loading Dock Container } \\
\text { Shipping screen and verify no access allowed. }\end{array}$ & 0 & OPEN & & & \\
\hline & & & 10 & $\begin{array}{l}\text { Attempt to enter the Processing List Additions } \\
\text { screen and verify no access allowed. }\end{array}$ & 0 & OPEN & & & \\
\hline & & & 11 & $\begin{array}{l}\text { Attempt to enter the Process List screen and } \\
\text { verify no access allowed. }\end{array}$ & 0 & OPEN & & & \\
\hline & & & 12 & $\begin{array}{l}\text { Attempt to enter the AS/RS Storage Pick List } \\
\text { screen and verify no access allowed. }\end{array}$ & 0 & OPEN & & & \\
\hline & & & 13 & $\begin{array}{l}\text { Attempt to enter the AS/RS Shipping Pick List } \\
\text { screen and verify no access allowed. }\end{array}$ & 0 & OPEN & & & \\
\hline & & & 14 & $\begin{array}{l}\text { Attempt to enter the Radiological Inventory } \\
\text { Summary screen and verify no access allowed. }\end{array}$ & 0 & OPEN & & & \\
\hline & & & 15 & $\begin{array}{l}\text { Attempt to enter the Processed Waste Data } \\
\text { Review/Modification screen and verify no. } \\
\text { access allowed. }\end{array}$ & 0 & OPEN & & & \\
\hline & & & 16 & $\begin{array}{l}\text { Attempt to enter the Location Table screen and } \\
\text { verify no access allowed. }\end{array}$ & 0 & OPEN & & & \\
\hline & & & 17 & $\begin{array}{l}\text { Attempt to enter the Profile Isotopic screen and } \\
\text { verify no access allowed. }\end{array}$ & 0 & OPEN & & & \\
\hline & & & 18 & $\begin{array}{l}\text { Attempt to enter the Route Description Table } \\
\text { screen and verify no access allowed. }\end{array}$ & 0 & OPEN & & & \\
\hline
\end{tabular}




\begin{tabular}{|c|c|c|c|c|c|c|c|c|c|}
\hline PROC \# & $\begin{array}{l}\text { PROCEDURE } \\
\text { NAME }\end{array}$ & $\underset{\#}{\text { CASE }}$ & $\begin{array}{c}\text { STE } \\
\mathbf{P} \\
\not f \\
\end{array}$ & STEP NAME & $\underset{\#}{\operatorname{REV}}$ & $\begin{array}{c}\text { PASS } \\
\text { 1st } \\
\text { TEST }\end{array}$ & $\begin{array}{l}\text { PASS } \\
\text { RETEST }\end{array}$ & COMMENTS & $\begin{array}{c}\text { SIGNOF } \\
\text { F } \\
\text { DATE }\end{array}$ \\
\hline \multirow[t]{11}{*}{ DMS-F81 } & \multirow[t]{11}{*}{ System Security } & \multirow[t]{11}{*}{02} & 19 & $\begin{array}{l}\text { Attempt to enter the Shipping Pick List Type } \\
\text { Table screen and verify no access allowed. }\end{array}$ & 0 & OPEN & & & \\
\hline & & & 20 & $\begin{array}{l}\text { Attempt to enter the SIE Isotopic Name Table } \\
\text { screen and verify no access allowed. }\end{array}$ & 0 & OPEN & & & \\
\hline & & & 21 & $\begin{array}{l}\text { Attempt to enter the TRU Container Code } \\
\text { Table screen and verify no access allowed. }\end{array}$ & 0 & OPEN & & & \\
\hline & & & 22 & $\begin{array}{l}\text { Attempt to enter the Field Analysis Type Table } \\
\text { screen and verify no access allowed. }\end{array}$ & 0 & OPEN & & & \\
\hline & & & 23 & $\begin{array}{l}\text { Attempt to enter the Material Table screen and } \\
\text { verify no access allowed. }\end{array}$ & 0 & OPEN & & & \\
\hline & & & 24 & $\begin{array}{l}\text { Attempt to enter the Wrap Miscellaneous Code } \\
\text { Table screen and verify no access allowed. }\end{array}$ & 0 & OPEN & & & \\
\hline & & & 25 & $\begin{array}{l}\text { Attempt to enter the Laboratory Analysis Table } \\
\text { screen and verify no access allowed. }\end{array}$ & 0 & OPEN & & & \\
\hline & & & 26 & $\begin{array}{l}\text { Attempt to enter the Sample Container Table } \\
\text { screen and verify no access allowed. }\end{array}$ & 0 & OPEN & & & \\
\hline & & & 27 & $\begin{array}{l}\text { Attempt to enter the Sample Matrix Table } \\
\text { screen and verify no access allowed. }\end{array}$ & 0 & OPEN & & & \\
\hline & & & 28 & $\begin{array}{l}\text { Attempt to enter the Sampling Method Table } \\
\text { sareen and verify no access allowed. }\end{array}$ & 0 & OPEN & & & \\
\hline & & & 29 & $\begin{array}{l}\text { Attempt to enter the Treatment Procedure } \\
\text { Table screen and verify no access allowed. }\end{array}$ & 0 & OPEN & & & \\
\hline
\end{tabular}




\begin{tabular}{|c|c|c|c|c|c|c|c|c|c|}
\hline PROC \# & $\begin{array}{l}\text { PROCEDURE } \\
\text { NAME }\end{array}$ & $\underset{\#}{\text { CASE }}$ & $\begin{array}{c}\text { STE } \\
\mathbf{P} \\
\# \\
\end{array}$ & STEP NAME & $\underset{\#}{\mathbb{R E V}}$ & $\begin{array}{c}\text { PASS } \\
\text { 1st } \\
\text { TEST }\end{array}$ & $\begin{array}{c}\text { PASS } \\
\text { RETEST }\end{array}$ & COMMENTS & $\begin{array}{c}\text { SIGNOF } \\
\text { F } \\
\text { DATE }\end{array}$ \\
\hline \multirow[t]{9}{*}{ DMS-F81 } & \multirow[t]{9}{*}{ System Security } & \multirow[t]{6}{*}{02} & 30 & $\begin{array}{l}\text { Attempt to enter the Error Message Table } \\
\text { screen and verify no access allowed. }\end{array}$ & 0 & OPEN & & & \\
\hline & & & 31 & $\begin{array}{l}\text { Attempt to enter the User Table screen and } \\
\text { verify no access allowed. }\end{array}$ & 0 & OPEN & & & \\
\hline & & & 32 & $\begin{array}{l}\text { Attempt to enter the Role Table screen and } \\
\text { verify no access allowed. }\end{array}$ & 0 & OPEN & & & \\
\hline & & & 33 & $\begin{array}{l}\text { Altempt to enter the Screen Table screen and } \\
\text { verify no access allowed. }\end{array}$ & 0 & OPEN & & & \\
\hline & & & 34 & $\begin{array}{l}\text { Attempt to enter the User Role Table screen } \\
\text { and verify no access allowed. }\end{array}$ & 0 & OPEN & & & \\
\hline & & & 35 & $\begin{array}{l}\text { Attempt to enter the Role Screen Table screen } \\
\text { and verify no access allowed. }\end{array}$ & 0 & OPEN & & & - \\
\hline & & \multirow[t]{3}{*}{03} & 1 & $\begin{array}{l}\text { Logon to DMS system as a user with test role } \\
\text { READ privileges. }\end{array}$ & 0 & OPEN & & & \\
\hline & & & 2 & $\begin{array}{l}\text { Enter the Receiving screen and verify that } \\
\text { query for data is allowed and commit of data } \\
\text { to the database is allowed by a user with } \\
\text { UPDATE access. }\end{array}$ & 0 & OPEN & & & \\
\hline & & & 4 & $\begin{array}{l}\text { Enter the NDE sereen and verify that query for } \\
\text { data is allowed and commit of data to the } \\
\text { database is allowed by a user with UPDATE } \\
\text { access. }\end{array}$ & 0 & OPEN & & & \\
\hline
\end{tabular}




\begin{tabular}{|c|c|c|c|c|c|c|c|c|c|}
\hline PROC \# & $\begin{array}{l}\text { PROCEDURE } \\
\text { NAME }\end{array}$ & $\underset{\#}{\text { CASE }}$ & $\begin{array}{c}\text { STE } \\
\mathbf{P} \\
\# \\
\end{array}$ & STEP NAME & $\underset{\#}{\text { REV }}$ & $\begin{array}{c}\text { PASS } \\
\text { 1st } \\
\text { TEST } \\
\end{array}$ & $\begin{array}{c}\text { PASS } \\
\text { RETEST }\end{array}$ & COMMENTS & $\begin{array}{c}\text { SIGNOF } \\
F \\
\text { DATE } \\
\end{array}$ \\
\hline \multirow[t]{5}{*}{ DMS-F81 } & \multirow[t]{5}{*}{ System Security } & \multirow[t]{5}{*}{03} & 5 & $\begin{array}{l}\text { Enter the NDA screen and verify that query } \\
\text { for data is allowed and commit of data to the } \\
\text { database is allowed by a user with UPDATE } \\
\text { access. }\end{array}$ & 0 & OPEN & & & \\
\hline & & & 7 & $\begin{array}{l}\text { Enter the LLW Sorting Table sereen and verify } \\
\text { that query for data is allowed and commit of } \\
\text { data to the database is allowed by a user with } \\
\text { UPDATE access. }\end{array}$ & 0 & OPEN & & & \\
\hline & & & 8 & $\begin{array}{l}\text { Attempt to enter the LLW Exit Glovebox } \\
\text { screen and verify that query for data is allowed } \\
\text { and commit of data to the database is allowed } \\
\text { by a user with UPDATE acce }\end{array}$ & 0 & OPEN & & - & . \\
\hline & & & 9 & $\begin{array}{l}\text { Enter the Loading Dock Container Shipping } \\
\text { screen and verify that query for data is allowed } \\
\text { and commit of data to the database is allowed } \\
\text { by a user with UPDATE a }\end{array}$ & 0 & OPEN & & . & \\
\hline & & & 11 & $\begin{array}{l}\text { Enter the Process List screen and verify that } \\
\text { query for data is allowed and commit of data } \\
\text { to the database is allowed by a user with } \\
\text { UPDATE access. }\end{array}$ & 0 & OPEN & & & \\
\hline \multirow[t]{2}{*}{ DMS-F91 } & \multirow[t]{2}{*}{ System Performance } & \multirow[t]{2}{*}{01} & 1 & $\begin{array}{l}\text { Execute Dyacomm script } 191.01 \mathrm{u} \text { to create } \\
\text { receipt data. }\end{array}$ & 0 & $\mathrm{x}$ & & & $4 / 10 / 96$ \\
\hline & & & 2 & $\begin{array}{l}\text { Log onto the DMS test database as USER } 1 \\
\text { and select Receiving from the main menu. }\end{array}$ & 0 & $\mathrm{x}$ & & & $4 / 10 / 96$ \\
\hline
\end{tabular}

